



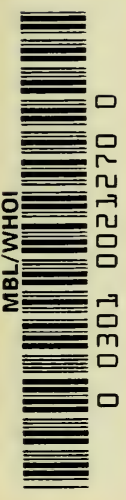





\section{Guide to the}

Classification and Identification of the ACTINOMYCETES and their ANTIBIOTICS 

GUIDE TO THE CLASSIFICATION

AND IDENTIFICATION OF THE

\section{Actinomycetes and their Antibiotucs}

by SELMAN A. WAKSMAN

Professor of Microbiology, Rutgers University

and HUBERT A. LECHEVALIER

Assistant Professor of Microbiology, Rutgers University

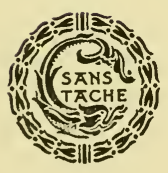

THE WILLIAMS \& WILKINS COMPANY Baltimore. 1953 
Copyright, 1953

The Williams \& Wilkins Company

Made in the United States of America

\author{
LIBRARY OF CONGRESS \\ CATALOG CARD NUMBER \\ 53-11919
}

Compoged and Printed at the

WAVERLY PRESS, INC.

Baltmohn 2, MD., U.S.A. 


\section{Preface}

Barely four years ago, an effort was made to summarize the existing knowledge of the nature and physiology of the actinomycetes in a book entitled "The Actinomycetes." No attempt was made to include in that volume any detailed system of classification or species characterization of these organisms, since it was felt that Bergey's Manual was quite sufficient for that purpose. Nor was any attempt made to characterize in detail the antibiotics produced by actinomycetes, since the available information was rather limited at that time.

The rapid progress made during the last few years in the screening of actinomycetes, especially of species belonging to the genus Streptomyces, for their ability to produce antibiotics, the use of many of these antibiotics for chemotherapeutic purposes, and the addition of numerous descriptions of new species of actinomycetes necessitate a more detailed presentation of the systematic position and identification of these organisms and of the antibiotics produced by them. In describing these new organisms, many investigators have been unable to identify their newly isolated cultures with those described in the last edition of Bergey's Manual, with the result that they preferred to give their cultures new names, often without regard to the established types. These new descriptions are found in various journals and even in patents. This guide has been prepared for the primary purpose of making this information available to workers in this field. Those species which have been insufficiently described or which appear to be synonyms are not listed in the guide, but will be found in the new edition of Bergey's Manual, now in the process of preparation.

In the preparation of this guide, advantage has been taken of progress made in the classification of the actinomycetes during the last 30 years, thanks largely to the work of Orskov, Jensen, Waksman and Henrici, Erikson, and Krassilnikov. Much, however, still remains to be done, especially on the physiology and biochemistry of actinomycetes. This is true particularly of one group of metabolic products, namely, the antibiotics. Because these substances have become so vitally important, an effort has been made to list all the antibiotics so far isolated, either in crude or in pure form, and to give their salient properties and uses.

This guide has been prepared with the primary purpose of facilitating the work of the student of antibiotics produced by actinomycetes and to help him in the identification of the organisms producing these antibiotics, as well as of the antibiotics themselves.

The authors want to express their sincere appreciation to Mrs. Herminie B. Kitchen for her editorial assistance in the preparation of this volume and for her painstaking help in its proofreading. 



\section{Contents}

Preface.......................... v

\section{Part I. The Actinomycetes}

By Selman A. Waksman

Classification of the Actinomycetes

Introduction... ............................. 1

The Genus Streptomyces. . . .................. 2

Need for Standard Media and Standard Conditions of Growth... . 4

Classification System of Krassilnikov................ 6

Classification System of Waksman and Henrici........... 9

Classification of Certain Important Group-Species of Streptomyces

from the Point of View of Antibiotic Production.......... 20

The Genus Nocardia........................... 29

Classification System of Krassilnikov ................ 29

Classification System of Waksman and Henrici........... 32

The Genus Micromonospora...................... 35

Classification System of Krassilnikov................ 35

Classification System of Jensen (emend.) . . . . . . . . . 36

The Genus Thermoactinomyces..................... 36

The Genus Actinomyces........................ 37

Description of Species of Streptomyces................. 38

Description of Species of Micromonospora................ 127

Description of Species of Thermoactinomyces.............. 130

Description of Species of Nocardia . . . . . . . . . . . . . . 132

Description of Species of Actinomyces................. 161

\section{Part II. The Antibiotics}

By Selman A. Waksman and Hubert A. Lechevalier

Production of Antibiotics by Actinomycetes.............. 165

Antibiotic Groups............................. 168

Key I to the Identification of the Antibiotics of Actinomycetes. . . 170

Key II to the Identification of the Antibiotics of Actinomycetes... 178

Description of the Antibiotics of Actinomycetes............ 185

Selected General References...................... 237

Index to Species of Actinomycetes.................. 241

Index to Antibiotics of Actinomycetes............... 245 



\section{PAR T I}

\section{The Actinomycetes}

By Selman A. Waksman

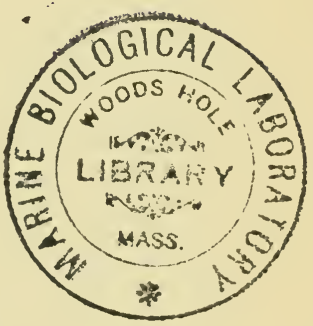





\section{Classification \\ of the Actinomycetes}

\section{Introduction}

Two important factors must be recognized in any attempt to classify the actinomycetes, especially for the purpose of establishing specific differences. These are, first, the ability of the organisms to produce vegetative and aerial mycelium; and second, the great variability of the cultures. To facilitate recognition of these organisms and to establish differences for classification purposes, well-defined media and standard conditions of cultivation must be used.

The filamentous growth and the true branching of the actinomycetes differentiate these organisms from the true bacteria. It is often difficult if not impossible to distinguish between the profuse branching of certain mycobacteria and the short-lived mycelium of the nocardias, except for the fact that the nocardias produce mycelium consistently in the early stages of their development. There is thus a gradual transition between the mycobacteria and the nocardias. There is also a definite transition between the nocardias and the streptomycetes, due to the constant and marked nature of the aerial mycelium of the latter and the transitory and undifferentiated aerial mycelium of the former.

In differentiating a nocardia from a streptomyces, one must consider the following differences: 1 . A nocardia usually does not produce aerial mycelium, and if it does, such mycelium is no different from the vegetative mycelium produced by the organism and never forms spirals; a streptomyces, on the other hand, produces a characteristic aerial mycelium, a property which may be lost, however, on continued cultivation or under special conditions, and such mycelium frequently forms characteristic spirals. 2. A nocardia multiplies by concentration and segmentation of the protoplasm within a filamentous cell, followed by dissolution of the cell membrane, the fragmented portions of the mycelium usually developing into fresh mycelium under favorable conditions, either by germ tubes or by lateral budding; a streptomyces produces true spores or conidia, the vegetative mycelium not segmenting spontaneously into bacillary or coccoid forms, but remaining nonseptate and coherent even in old cultures, thus producing the characteristic tough-textured, leathery growth. 3 . In a nocardia, the aerial mycelium represents an extension upward of the vegetative mycelium; it does not exhibit any differentiated protoplasm and 
is sterile and abortive; on the other hand, when a streptomyces has lost the capacity of producing aerial mycelium, a form analogous to that of a nocardia may result, except for the structure of the mycelium and the capacity of the degenerated streptomyces to regain the lost capacity. 4 . Another difference between the nocardias and the streptomycetes is the acid-fastness or partial acid-fastness of some of the former when grown in certain media.

The differentiation between species of Streptomyces, and those of Micromonospora is not very difficult, since both formation of aerial mycelium and sporulation are markedly distinct for both genera. Some difficulty may be experienced in differentiating species of these two genera from those of Thermoactinomyces, since the latter also produce aerial mycelium, similar to species of Streptomyces, and they produce single spores, similar to Micromonospora, but such spores are produced in the aerial mycelium rather than in the vegetative mycelium as in the case of the Micromonospora.

\section{Classification of Actinomycetes According to Bergey}

According to the latest edition of Bergey's Manual, the order ACTINOMYCETALES is divided into three families, Mycobacteriaceae, Actinomycetaceae, and Streptomycetaceae. The first family comprises the genus Mycobacterium, which is beyond the scope of this treatise. The second and third families comprise the true actinomycetes, with which this treatise is primarily concerned. The genera Nocardia and Actinomyces fall into the second family, and the genera Streptomyces and Micromonospora belong to the third. Another genus has recently been added, Thermoactinomyces, comprising largely certain thermophilic forms.

Of the various species belonging to the five genera of true actinomycetes, those of the genus Actinomyces have not, so far, been found capable of producing antibiotics. Among the species belonging to the other four genera, those of the genus Streptomyces are by far the most important; hence a detailed consideration of the nature of this genus is justified.

\section{The Genus Streptomyces}

Species of Streptomyces produce a well-developed mycelium. The diameter of the hyphae seldom exceeds $1.0 \mu$ and is usually only $0.7-0.8 \mu$. The hyphae vary greatly in length. Some are long with limited branching; others are short and much branched. The vegetative mycelium does not form cross walls; it does not break up into rod-shaped and coccus-like bodies. The streptomycetes reproduce by means of conidia or by bits of mycelium. The spores or conidia are formed in special spore-bearing hyphae or sporophores which arise from the aerial mycelium, either monopodially or in the form of tufts or whorls. The sporulating hyphae are straight or 
curved. The curvatures range from mere waviness to the formation of perfect spirals, which may be compact, in the form of fists, or long and loose.

The growth of streptomyces "colonies" on artificial media is smooth or lichnoid, hard and densely textured, raised, and adhering to the medium. The colony is usually covered completely or partially, in the form of spots or concentric rings, by aerial mycelium, which may be variously pigmented, depending on the species and on the composition of medium. In liquid media, especially in shaken cultures, growth of a streptomyces is usually in the form of flakes, which gradually fill the container, or in the form of spherical growths or puffballs. It is the former type of growth that is most desirable from the point of view of antibiotic production.

Many of the cultures, either in the form of colonies on the surface of solid media or as flaky growth in submerged culture, may undergo rapid autolysis. The production of antibiotics usually corresponds with the lysis of the cultures. Frequently the lysis is brought about by a phage, actinophage, which is injurious because of the premature destruction of the mycelium.

The analogy between the aerial mycelium and sporulation of species of Streptomyces and those of certain fungi has frequently led to confusion in the classification of actinomycetes as a whole. More recent evidence, notably the sensitivity of actinomycetes to antibiotics and to specific phages, as well as the nature of their circumscribed growth, tends to place the actinomycetes as a group, including the genus Streptomyces, closer to the true bacteria than to the true fungi.

Finally a word must be said about the "physiological races" of the streptomycetes. These organisms undergo in culture certain qualitative and quantitative variations. The streptomycin-producing strain of S. griseus, for example, has yielded two types of variants, one of which produced no aerial mycelium and formed no streptomycin, and the other of which produced a red pigmented vegetative mycelium aud formed another antibiotic (rhodomycetin) but not streptomycin. The qualitative differences of the variants are best expressed in the amount of antibiotic produced, which may vary as much as a hundredfold for the same species. From the point of view of antibiotic production, the following five subgroups of S. griseus may now be recognized: (a) those that produce streptomycin (as well as certain other antibiotics, such as cycloheximide and streptocin), (b) those that produce grisein, (c) those that produce candicidin, (d) those that produce still unidentified antibiotics, and (e) those that do not yield any antibiotic, at least under the particular condition of culture. Whether all of these variants have originated from a single original species of $S$. griseus is a matter of speculation. The limited evidence seems to point to the affirmative. 
Because of these variations among strains of a single species, it has often been difficult to identify freshly isolated cultures with already established types. This has been particularly difficult when the latter were known by published description only. But even when type cultures were available for comparison, they may have undergone such marked changes as a result of long cultivation in artificial media that they no longer represent the original isolate. This may be illustrated again with S. griseus. When the original strain of this culture, which was isolated in our laboratory in 1915, was tested in 1946 for antibiotic formation, it was found to be unable to produce any antibiotic. When the same culture, which was deposited in the Baarn (Holland) Collection in 1920, was tested, it was found to be able to form an antibiotic that was not streptomycin. When the original culture kept in our collection was irradiated by Kellner, a strain that was capable of producing streptomycin was obtained. One may, therefore, only speculate whether this proves that the original 1915 strain possessed the capacity of producing streptomycin and lost it, or that it did not possess it at all, but on irradiation produced a mutant that acquired such capacity.

\section{Need for Standard Media and Standard Conditions of Growth}

The use of standard media, comprising both synthetic and complex organic media, for the growth of actinomycetes is particularly important in any attempt to characterize and identify these organisms. The same is true of the need for a standard environment, notably aeration and temperature control. Synthetic media have found extensive application in the study of the morphology and physiology of these organisms. This is true especially for cultural characterization of these organisms, particularly pigmentation. Organic media, often quite specific, are used for obtaining supplementary evidence, especially for strains that do not grow at all or grow only very weakly on the common inorganic media.

The great majority of actinomycetes are aerobic, very few are anaerobic, many are microaerophilic. To supply proper aeration, the organisms are grown on the surface of solid media, or in shallow liquid media, or in a thoroughly aerated submerged condition.

Temperatures of $25^{\circ}-30^{\circ}$ are usually used for incubation of the great majority of streptomycetes, nocardias, and micromonospora. Frequently somewhat different temperatures are used for seed production and for antibiotic formation under factory conditions. Pathogenic organisms require $37^{\circ} \mathrm{C}$, and thermophiles need $50^{\circ}-60^{\circ} \mathrm{C}$ or even higher temperatures.

Among the stable morphological properties of actinomycetes essential for purposes of characterization and classification, one must list the structure and subsequent changes in the vegetative mycelium, the production and 
nature of the aerial mycelium, the nature of the sporulating branches or sporophores, and the nature of the spores.

Among the physiological and cultural properties essential for characterization purposes, pigmentation of the vegetative and aerial mycelium is most important; the formation of soluble pigments, both in synthetic and in complex organic media, is also significant. Am.ng the other characteristics, one may list utilization of starch (diastase fo: nation), various proteins (protease), sucrose (invertase), cellulose (cellulase?), and a variety of other carbon compounds. Reduction of nitrate, production of odor, and coagulation of milk (acid production) are among the other characteristics. Still other activities include the utilization of fats, paraffin, and phenol.

The various fermentation properties, comprising the utilization of sugars and related compounds, with and without the formation of acids, can supply additional information for species differentiation. The antagonistic activities and the ability to produce antibiotics have recently come into popular use for the description of actinomycetes. Their sensitivity to phages and to known antibiotics is also of considerable importance in establishing specific differences.

Many species of actinomycetes have been described in the literature as pathogenic to plants or animals. The fact that such cultures were isolated from infectious diseases is no proof, however, that they are the causative agents of such diseases.

Many of the earlier and even some of the more recent descriptions of actinomycetes are superficial, and it is often difficult to compare the characteristics of a freshly isolated culture with those of the published description. No wonder, therefore, that it has been much easier to give a fresh culture a new specific name rather than to attempt its identification with a species already described.

In identifying cultures of actinomycetes, extensive use has been made, therefore, of their morphological and physiological properties. The cultural characteristics, notably growth characteristics on various organic and synthetic media, formation of soluble pigments, proteolytic and diastatic properties, utilization and transformation of specific organic and inorganic compounds, have received particular attention. Pridham and Gottlieb emphasized recently that the utilization of carbon compounds should receive greater consideration for species determination than hitherto. All the particular species tested were able to utilize $d$-glucose, $d$-mannose, dextrin, and glycerol, but not erythritol, phenol, cresols and the sodium salts of formic, oxalic, and tartaric acids. The utilization of such compounds, however, as rhamnose, raffinose, xylose, lactose, mannitol, dulcitol, inositol, and the sodium salts of acetic and succinic acids, was selective.

It has been suggested that rather than single species, group species should 
be recognized, as will be shown later. Thus, one could recognize such wellestablished types as S. albus, S. griseus, S. lavendulae, S. flavus, S. coelicolor, and certain others. The various species within a group could be considered as related to a given type. This is possible where a large number of isolates are available.

Numerous systems for the classification of the various groups of actinomycetes have been proposed. Two of these are most comprehensive and are, therefore, presented here. One is that of Krassilnikov, and the other, that of Waksman and Henrici, as included in Bergey's Manual.

\section{Classification System of Krassilnikov}

A. Sporophores branching monopodially.

I. Spiral-shaped sporophores, produced on hyphae of aerial mycelium. 1. Spores spherical or oval.

a. Cultures colorless, not producing any pigmentation. $a^{2}$. Aerial mycelium white.

$a^{2}$. Saprophytes, living on dead material.

Streptomyces albus

$b^{2}$. Parasites.

$a^{3}$. Living in bodies of men and animals.

Streptomyces bovis

$\mathrm{b}^{3}$. Living on plants.

Streptomyces totschidlowskii

$b^{1}$. Aerial mycelium dark gray.

Streptomyces griseus

$c^{1}$. Aerial mycelium green.

Streptomyces glaucus

b. Cultures pigmented blue.

$a^{1}$. Pigment of the anthocyanin type, similar to litmus.

Streptomyces coelicolor

$b^{1}$. Blue pigment not changing with acidity of medium.

Streptomyces cyaneus

c. Cultures violet, forming two basic pigments (red and blue), both dissolved into the substrate.

$a^{1}$. Cultures not forming any fluorescent substance in liquid media.... Streptomyces violaceus

$b^{1}$. Cultures producing in synthetic media a fluorescent substance of blue-green color similar to pyocyanin.

Streptomyces pluricolor

d. Cultures black-violet, forming red and blue pigments, as well 
as a brown pigment of the type of melanin, which changes the violet color of the culture to violet-black.

Streptomyces violaceus-niger

e. Cultures red-colored, producing pigments insoluble in water, of the lipoactinochrome type; color of medium not changing with acidity.

$\mathrm{a}^{1}$. Cultures not forming any brown or black pigments; they are always red, sometimes with a brownish tinge but not black.

$a^{2}$. Saprophytes.

$b^{2}$. Parasites.

\section{Streptomyces ruber}

Streptomyces madurae

$b^{1}$. Cultures producing on synthetic media, in addition to pigments, a black or dark brown substance which gives the culture a red-brown to black color.

Streptomyces melanocyclus

f. Cultures yellow, citron-yellow, or brownish yellow. $a^{1}$. Saprophytes.

$b^{1}$. Parasites.

$$
\text { Streptomyces flavus }
$$

$a^{2}$. Living in bodies of men and warm-blooded animals.

Streptomyces hominis

$\mathrm{b}^{2}$. Living in bodies of cold-blooded animals.

Streptomyces lacertae

$c^{2}$. Living on plants.

Streptomyces setonii

g. Cultures orange.

$a^{1}$. Saprophytes.

Streptomyces aurantiacus

$b^{1}$. Parasites. Streptomyces phenotolerans

h. Cultures green or brownish green.

Streptomyces viridochromogenes

i. Cultures black, producing a pigment of the melanin type.

Streptomyces niger

j. Cultures pigmented dark brown, but not black.

$a^{1}$. Saprophytes.

Streptomyces chromogenes

$b^{1}$. Parasites. .Streptomyces gracilis

2. Spores cylindrical or elongated. 
a. Cultures colorless.

Streptomyces longisporus

b. Cultures red, sporophores mostly straight. $a^{1}$. Saprophytes.

Streptomyces longisporus-ruber

$b^{1}$. Parasites.

$\mathrm{a}^{2}$. Living in bodies of men and animals.

Streptomyces spumalis

$\mathrm{b}^{2}$. Living on plants.

Streptomyces salmonicolor

c. Cultures orange.

Streptomyces fradiae

d. Cultures yellow. $a^{1}$. Saprophytes.

Streptomyces longisporus-flavus

$b^{1}$. Parasites living on plants.

Streptomyces scabies

e. Cultures citron-yellow.

f. Cultures green.

Streptomyces virgatus

Streptomyces viridans

g. Cultures brown or chocolate color.

h. Cultures black.

Streptomyces halstedii

Streptomyces nigrificans

II. Sporophores straight or wavy, but not spiral.

1. Spores produced by means of fragmentation of plasma within cells.

a. Spores spherical or oval.

$a^{1}$. Cultures colorless.

$b^{1}$. Cultures green.

Streptomyces globisporus

$a^{2}$. Saprophytes.

Streptomyces viridis

$b^{2}$. Parasites.

Streptomyces cretaceus

$c^{1}$. Cultures brown.

Streptomyces globosus

b. Spores cylindrical or elongated.

$a^{1}$. Cultures colorless.

Streptomyces candidus

$\mathrm{b}^{1}$. Cultures pigmented. 
2. Spores produced by means of segmentation of aerial hyphae.

a. Cultures colorless

Streptomyces farinosus

b. Cultures pigmented red.

Streptomyces oidiosporus

c. Cultures yellow-orange.

Streptomyces longissimus

d. Cultures brown.

Streptomyces fumosus

B. Sporophores produced in whorls.

I. Sporophores straight.

Streptomyces verticillatus

II. Sporophores spiral-shaped.

1. Spores spherical, oval.

Streptomyces reticuli

2. Spores cylindrical, elongated.

Streptomyces circulatus

\section{Classification System of Waksman and Henrici}

A. Saprophytes; psychrophilic to mesophilic.

I. Soluble pigment on organic media absent, or faint brown, pink, purple, golden yellow, or blue.

a. Pigment absent, or only faint brown pigment produced in protein media.

a. Aerial mycelium abundant, white.

$a^{1}$. Spirals formed; spores spherical to ellipsoidal.

1. Streptomyces albus

$b^{1}$. Long, open spirals; spores cylindrical.

2. Streptomyces longisporus

$\mathrm{c}^{1}$. Straight sporophores, forming broom-shaped clusters; spores spherical to oval.

3. Streptomyces globisporus

b. Aerial mycelium whitish to light gray, in concentric zones.

4. Streptomyces annulatus

c. Aerial mycelium on synthetic sucrose agar sandy lavender to dark gray.

\section{Streptomyces rochei}

2. Aerial mycelium gray to blue-gray; soluble pigment blue.

a. Strongly proteolytic.

$a^{1}$. Spirals formed.

$a^{2}$. Pigment at first red, changing to blue.

6. Streptomyces coelicolor. 
$b^{2}$. Pigment at first yellow-red, changing to blue or bluish green.

7. Streptomyces pluricolor.

$c^{2}$. Pigment unchanged with acidity.

8. Streptomyces cyaneus

$b^{1}$. No spirals formed.

9. Streplomyces vinaceus

b. Weakly proteolytic; aerial mycelium poorly developed.

10. Streptomyces violaceus

3. Pigment at first green, becoming brown.

a. Aerial mycelium usually absent.

11. Streptomyces verne

b. Aerial mycelium dark gray, olive-colored, or gray-green.

12. Streptomyces viridans

4. Growth pink on synthetic media, yellowish on organic media; no soluble pigment.

13. Streptomyces californicus

5. Growth yellow to greenish or orange-colored; soluble pigment yellow to golden yellow.

a. Growth yellow to green; pigment insoluble.

14. Streptomyces virgatus

b. Growth sulfur-yellow; soluble pigment yellow.

$a^{1}$. Conidia oval to elliptical.

$a^{2}$. Aerial mycelium white.

15. Streptomyces flaveolus

$b^{2}$. Aerial mycelium light yellow.

16. Streptomyces parvus

$c^{2}$. Aerial mycelium white to gray to reddish gray.

17. Streptomyces xanthophaeus

$b^{1}$. Conidia spherical; cellulose decomposed.

18. Streptomyces cellulosae

c. Growth reddish brown to orange-colored to cinnamon-drab, covered with white to gray aerial mycelium; soluble pigment on synthetic media yellowish.

$a^{1}$. No soluble pigment on gelatin. No peptonization of milk.

19. Streptomyces rimosus

$b^{1}$. Faint yellowish coloration of liquefied gelatin. Milk rapidly peptonized.

20. Streptomyces griseoflavus

$\mathrm{c}^{1}$. Soluble pigment golden yellow.

21. Streptomyces aureofaciens 
d. Growth cream-colored to brown; aerial mycelium white to yellowish.

$a^{1}$. Soluble pigment yellow to yellow-orange.

22. Streptomyces albidoflavus

$b^{1}$. Soluble pigment yellowish to yellow-green.

$a^{2}$. Aerial mycelium white to yellow.

23. Streptomyces lieskei

$\mathrm{b}^{2}$. Aerial mycelium gray.

$a^{8}$. Growth on synthetic agar yellowish green.

24. Streptomyces flavovirens

$\mathrm{b}^{3}$. Growth on synthetic agar yellow; produces soluble yellow pigment on calcium-malate-glycerol agar.

24a. Streptomyces celluloflavus

$c^{8}$. Growth on glucose-asparagine agar yellow, becoming black.

25. Streptomyces limosus

6. Growth cream-colored; soluble pigment yellowish brown to reddish brown.

26. Streptomyces griseoluteus

7. Growth coral-red; aerial mycelium scant, white; soluble pigment brown.

a. Gelatin rapidly liquefied.

27. Streptomyces bobiliae

b. Gelatin slowly liquified.

28. Streptomyces aurantiacus

8. Growth on synthetic media mouse-gray; aerial mycelium white to gray.

a. Sporophores straight.

29. Streptomyces griseolus

b. Sporophores broom-shaped.

30. Streptomyces fasciculus

9. Growth cream-colored to yellowish to red; aerial mycelium white to gray.

a. Growth becoming red; aerial mycelium white.

31. Streptomyces erythreus

b. Growth yellow; aerial mycelium mouse-gray to drab.

32. Streptomyces flavogriseus

10. Soluble pigment on potato plug brown to brownish red to reddish purple.

a. Growth on potato greenish-colored; spirals formed.

33. Streptomyces diastaticus 
b. Growth on potato gray; no spirals formed.

34. Streptomyces canescus

c. Growth on potato yellowish-colored. $a^{1}$. Greenish tinge produced.

35. Streplomyces fimicarius

$b^{1}$. Pigment reddish brown.

36. Streptomyces felleus

$\mathrm{c}^{1}$. Soluble brown pigment on synthetic agar.

37. Streptomyces achromogenes

d. Growth on potato pink to reddish purple; spirals produced.

38. Streptomyces noursei

II. Soluble pigment on organic media brown.

1. Pigment deep brown (chromogenic types).

a. Aerial mycelium thin, rose-colored.

$a^{1}$. Spirals produced.

39. Streptomyces roseochromogenus

$b^{1}$. No spirals formed.

40. Streptomyces cinnamonensis

b. Aerial mycelium gray to brown to reddish. $a^{1}$. Growth on organic media greenish to black.

41. Streptomyces olivochromogenus $b^{1}$. Growth dark brown.

42. Streptomyces resistomycificus

c. Aerial mycelium cottony, dark brown; rapid liquefaction of gelatin.

43. Streptomyces diastatochromogenes

d. Colonies yellow; aerial mycelium white to gray.

44. Streptomyces flavochromogenes

e. Growth white to gray; aerial mycelium white to gray.

45. Streptomyces bikiniensis

f. Growth white, fluffy; sporulating hyphae straight.

45a. Streptomyces mirabilis

g. Growth cream to brown-colored; aerial mycelium gray; sporophores in clusters.

46. Streptomyces antibioticus

h. Aerial mycelium white, turning light pink on certain media; sporophores straight, no spirals.

47. Streptomyces griseocarneus

i. Aerial mycelium greenish yellow, turning gray; sporophores produced in tufts or whorls.

48. Streptomyces viridoflavus 
2. Growth on synthetic agar dark brown.

a. Aerial mycelium dark gray; spores globose.

49. Streptomyces globosus

b. Aerial mycelium white; spores cylindrical.

50. Streptomyces cylindrosporus

3. Growth on synthetic agar dark green; aerial mycelium white to light green.

51. Streptomyces viridochromogenes

4. Pigment on synthetic agar deep brown to black.

a. Growth on potato orange to orange-red; growth on synthetic agar brown to purplish; no aerial mycelium.

52. Streptomyces purpeochromogenus

b. Growth on potato brown to black.

$\mathbf{a}^{2}$. Aerial mycelium on synthetic agar white to brownish.

53. Streptomyces phaeochromogenus

$b^{1}$. Growth on synthetic media colorless to light orange; aerial mycelium gray to cinnamon-drab.

54. Streptomyces aureus

5. Growth on synthetic agar grayish yellow; light yellow soluble pigment.

55. Streptomyces tanashiensis

6. Soluble pigment on synthetic media red to rose-red.

a. Soluble pigment on potato black.

56. Streptomyces erythrochromogenes

b. No soluble pigment on potato.

57. Streptomyces collinus

7. Aerial mycelium lavender to light tan or pink.

a. Aerial mycelium cottony white, lavender to vinaceous-lavender-colored.

58. Streptomyces lavendulae

b. Aerial mycelium light tan to pink.

59. Streptomyces venezuelae

c. Aerial mycelium grayish pink to lavender.

60. Streptomyces virginiae

8. Aerial mycelium forms whorls.

a. Growth gray-colored to brownish.

$a^{1}$. Spores spherical.

61. Streptomyces reticuli

$b^{1}$. Spores cylindrical.

62. Streptomyces netropsis 
b. Growth dark gray to gray-green; spores cylindrical.

63. Streptomyces verticillatus

c. Spores produced in spirals; spores cylindrical, weakly proteolytic.

64. Streptomyces circulatus

d. Whorls in secondary branches; growth yellowish red to pink.

65. Streptomyces rubrireticuli

9. Growth on potato greenish to black.

a. Growth on synthetic agar yellow to sulfur-yellow.

66. Streptomyces flavus

b. Growth on synthetic agar red to orange.

67. Streptomyces ruber

c. Growth on synthetic agar black.

68. Streptomyces niger

d. Growth on synthetic agar white.

69. Streptomyces alboniger

10. Growth on synthetic and organic media yellow; sporophores straight.

70. Streptomyces abikoensum

11. Soluble brown pigment only occasionally produced; growth usually colorless; odor very strong.

71. Streptomyces odorifer

III. No soluble pigment produced in organic media.

1. Proteolytic action strong.

a. Growth yellowish to orange-brown.

$\mathrm{a}^{1}$. Aerial mycelium white to rose-colored.

72. Streptomyces roseoflavus

$b^{1}$. Growth only on potato and serum yellowish; aerial mycelium white.

73. Streptomyces putrificans

b. Growth yellowish green to citron-yellow; aerial mycelium white to yellow to pinkish.

74. Streptomyces citreus

c. Growth golden yellow to orange-colored; soluble pigment yellow to orange.

$a^{1}$. Growth may also be red-brown and pigment orange.

75. Streptomyces fulvissimus

$b^{1}$. Growth yellow; aerial mycelium powdery white.

d. Growth colorless to cream-colored; aerial mycelium scanty, white.

76. Streptomyces chrysomallus 
$a^{1}$. Pigment on nutrient agar faint yellowish.

77. Streptomyces gougeroti

$b^{1}$. No soluble pigment; forms oidiospores.

78. Streptomyces farinosus

$\mathrm{c}^{1}$. Pigment on synthetic media yellowish.

79. Streptomyces albidus

e. Growth white to cream-colored; aerial mycelium flesh-colored to light cinnamon-colored.

80. Streptomyces cinnamoneus

f. Growth on synthetic media dark to black to almost bluish black; aerial mycelium white to gray.

$a^{1}$. Soluble pigment produced on autolysis.

81. Streptomyces violaceoniger

$b^{1}$. No soluble pigment.

82. Streptomyces gedanensis

g. Growth colorless to yellowish to olive-buff; aerial mycelium water-green.

83. Streptomyces griseus

h. Growth bright orange to golden red; aerial mycelium white to yellow.

84. Streptomyces longissimus

i. Growth yellow to olive-ocher; aerial mycelium mouse-gray.

85. Streptomyces olivaceus

j. Growth yellow; aerial mycelium scant or rose-yellow.

86. Streptomyces microflavus

k. Growth yellowish to brown to reddish brown.

$a^{1}$. Aerial mycelium white to mouse-gray.

87. Streptomyces cacaoi

2. Proteolytic action limited.

a. Soluble pigment produced on synthetic agar.

$a^{1}$. Pigment purple.

88. Streptomyces novaecaesareae

$b^{1}$. Pigment brown to black.

$a^{2}$. Growth on potato gray to brown.

89. Streptomyces exfoliatus

$b^{2}$. Growth on potato greenish to black.

90. Streptomyces gelaticus

$\mathrm{c}^{2}$. Aerial mycelium pigmented green.

91. Streptomyces glaucus

b. No soluble pigment on synthetic agar. 
$a^{1}$. Growth dark brown to almost black; aerial mycelium white to gray.

$a^{2}$. Abundant spirals in aerial mycelium.

$a^{3}$. Conidia spherical to oval.

92. Streptomyces rutgersensis

$\mathrm{b}^{3}$. Conidia oval to cylindrical.

93. Streptomyces halstedii

$b^{2}$. No spirals produced on synthetic agar.

$a^{3}$. Spores cylindrical; growth on synthetic agar dark brown.

94. Streplomyces fumosus

$b^{3}$. Spores oval.

a4. Growth on synthetic agar light brown.

95. Streptomyces lipmanii

$b^{1}$. Growth cream-colored to yellow or yellow-orange.

$a^{2}$. Aerial mycelium on certain media white, moist with dark, glistening patches; spores oval.

96. Streptomyces hygroscopicus

$b^{2}$. Aerial mycelium white-yellow to brownish yellow; spores cylindrical.

97. Streptomyces longisporoflavus

$c^{2}$. Aerial mycelium white; spores cylindrical.

98. Streptomyces candidus

$\mathrm{d}^{2}$. Aerial mycelium powdery white, with yellow tinge.

99. Streptomyces alboflavus

$\mathrm{e}^{2}$. Growth cream-colored; aerial mycelium cottony white; pigment in milk and on potato rose to yellowish.

100. Streptomyces flocculus

$\mathrm{f}^{2}$. Aerial mycelium gray; slight darkening of potato plug.

101. Streptomyces Leben, Stessel and Keitt

$\mathrm{c}^{1}$. Growth orange or red.

$a^{2}$. Growth yellowish to orange; aerial mycelium seashellpink.

102. Streptomyces fradiae

$b^{2}$. Growth rose to red; aerial mycelium white. $a^{3}$. Growth on gelatin yellow to red; slow liquefaction.

103. Streptomyces albosporeus

$b^{3}$. Growth light brown; rapid liquefaction.

104. Streptomyces purpurescens

$\mathrm{c}^{2}$. Growth light rose to purple-red; aerial mycelium 
whitish to rose-white; pigment dissolves in fat-containing media.

105. Streptomyces longispororuber

$d^{2}$. Growth pale rose to red; aerial mycelium weakly developed, velvety, rose-white.

106. Streptomyces oidiosporus

$\mathrm{e}^{2}$. Growth red; aerial mycelium black.

107. Streptomyces melanocyclus

3. Proteolytic action very weak.

a. Growth colorless; aerial mycelium whitish; acid-resistant.

108. Streptomyces acidophilus

b. Growth becoming salmon-pink; acid-sensitive.

109. Streptomyces rubescens

c. Growth green to dark green; aerial mycelium whitish to grayish; no soluble pigment.

110. Streptomyces viridis

B. Saprophytes; thermophilic.

I. Growth on potato yellowish; diastatic.

1. No aerial mycelium.

111. Streptomyces thermophilus

2. Aerial mycelium light gray.

110. Streptomyces thermodiastaticus

II. Growth on potato abundant, dark-colored; nondiastatic.

113. Streptomyces thermofuscus

III. Thermotolerant cultures.

\section{Streptomyces casei}

C. Plant parasites or cultures isolated from diseased plants or from soil in which diseased plants were grown (after Millard and Burr).

I. Isolated from potato scab or from soil in which scabby potatoes were grown.

1. Star-like colonies in glycerol synthetic solution.

a. Deep pigment produced in nearly all media; tyrosinase positive.

\section{Streptomyces clavifer}

b. Deep pigment produced in protein media only; tyrosinase positive.

\section{Streptomyces fimbriatus}

c. Pale pigment produced in nearly all media; tyrosinase negative.

\section{Streptomyces carnosus}

d. No soluble pigment (or only trace) produced; minute craters produced on sucrose and glucose media.

118. Streptomyces craterifer 
2. Surface growth heavy on glycerol synthetic solution with abundant aerial mycelium.

a. Aerial mycelium on nutrient potato agar limited, pale gray.

119. Streptomyces gracilis

b. Aerial mycelium on nutrient potato agar abundant, white; gelatin liquefaction stratiform; no soluble pigment.

120. Streptomyces praecox

c. Gelatin liquefaction stratiform; soluble pigment produced.

121. Streptomyces setonii

d. Abundant aerial mycelium on nearly all media; pigment on calcium malate-glycerol agar carnelian red.

122. Streptomyces praefecundus

3. Surface growth fair on glycerol synthetic solution with some aerial mycelium.

a. Soluble pigment (often green) produced on all solid media.

123. Streptomyces viridogenes

b. Soluble pigment (yellow) produced on all synthetic media.

124. Streptomyces loidensis

c. Soluble pigment none or only poor; decided clot produced in milk.

125. Streptomyces wedmorensis

4. Surface growth scant or none, but some bottom growth on glycerol synthetic solution.

a. Color changes produced in brom-cresol milk.

$a^{1}$. Tyrosinase positive; no aerial mycelium on nutrient potato agar.

126. Streptomyces scabies

$b^{1}$. Tyrosinase negative; aerial mycelium produced on all solid media.

$a^{2}$. Aerial mycelium on synthetic sucrose agar abundant. $a^{3}$. Growth good on egg-albumen agar with abundant aerial mycelium.

127. Streptomyces tenuis

$b^{3}$. Growth poor on egg-albumen agar with scant aerial mycelium.

128. Streptomyces marginatus

$b^{2}$. Aerial mycelium on synthetic sucrose agar scant; no true aerial mycelium produced on any media; colonies often show dark centers.

129. Streptomyces salmonicolor

b. No color changes produced in brom-cresol milk.

$a^{1}$. Facultative anaerobe; no true aerial mycelium, or only 
trace, produced on any media; colonies frequently show dark centers.

130. Streptomyces maculatus

$\mathrm{b}^{1}$. Obligate aerobe; aerial mycelium arises centripetally on the colonies.

131. Streptomyces coroniformis

5. No growth on glycerol synthetic solution, starch not hydrolyzed. a. Growth in brom-cresol milk good; characteristic color changes.

132. Streptomyces spiralis

6. Other organisms isolated from potato scab.

133. Streptomyces sampsonii

II. Grow on or isolated from sweet potatoes.

134. Streptomyces intermedius

1. Growth on synthetic agar olive-yellow; no aerial mycelium; no soluble pigment on potato.

135. Streptomyces ipomoea

2. Growth on synthetic agar cream-colored; growth on potato reddish brown with soluble pigment purple; aerial mycelium white.

136. Streptomyces poolensis

III. Attack or isolated from scab of mangels and sugar beets.

137. Streptomyces tumuli

D. Isolated from animal tissues; in the animal body, hyphae often show clavate enlargements at the ends.

I. Limited proteolytic action in gelatin, milk, coagulated egg-albumen, or fibrin.

1. Vegetative growth white.

a. No soluble pigment in organic media.

138. Streptomyces listeri

b. Soluble pigment in organic media brown to brown-red.

139. Streptomyces galtieri

2. Vegetative growth cream-colored; aerial mycelium scant, white.

140. Streptomyces upcottii

3. Growth very limited on various media, except on potato plug; no liquefaction of gelatin.

141. Streptomyces hortonensis

II. Strong proteolytic action in gelatin and milk.

1. Growth on potato plug moist, membranous.

a. Pigment deep brown.

142. Streptomyces beddardii 


\section{b. Pigment faint brown.}

143. Streptomyces kimberi

2. Growth on potato plug abundant, becoming black; aerial mycelium white-gray; plug discolored.

144. Streptomyces somaliensis

3. Growth on some media pink-colored.

145. Streptomyces panjae

4. Aerial mycelium on most media profuse, white; spiral formation. 146. Streptomyces willmorei

E. No aerial mycelium.

\section{Streptomyces sterilis group}

This group comprises forms that have lost the capacity to produce aerial mycelium. They can be subdivided into Streptomyces sterilis albus, Streptomyces sterilis ruber, Streptomyces sterilis viridis, Streptomyces sterilis flavus, etc.

\section{Classification of Certain Important Group-Species of Streptomyces, From Point of View of ANtibiotic Production}

Careful examination of the foregoing systems of classification of species of Streptomyces will reveal considerable overlapping of species. Frequently a given culture may be placed in one species or another, depending on the particular media and on the conditions used for growing the organism and the idiosyncrasies of the observer. Classification becomes particularly difficult when one bears in mind the marked variation frequently observed between different isolates of the same species and the tendency of individual cultures to mutate upon continued cultivation in artificial media. The fact that identifications are usually based upon comparisons with published descriptions rather than with type cultures has resulted in the tendency to create new species on the basis of minor differences.

These considerations have often led to emphasis upon group characterization rather than upon single culture-species. Several such groups, especially those comprising the more important antibiotic-yielding strains, may be selected for further examination. The individual species or strains within each of these groups vary greatly in their cultural and morphological properties, as well as in nature and concentration of the antibiotic produced.

Two of the groups selected for this comparison are typically chromogenic, being capable of producing brown to dark brown or almost black soluble pigments on protein-containing media, due largely to the formation of the enzyme tyrosinase ( $S$. antibioticus and $S$. lavendulae). One group is nonchromogenic, although it produces a faint soluble brown pigment on certain 
media; this pigment may be due to lysis of the growth, which in some strains or under certain conditions is olive-green to olive-buff (S. griseus). One group, comprising a large number of species, is characterized by yellow growth on various media and frequent production of a soluble yellow, yellow-orange, golden, or greenish yellow pigment (S. flavus). The fifth and sixth groups, which are also nonchromogenic, are characterized by the production of red to red-orange and light red growth, with poorly developed, white to rose-white aerial mycelium or well-developed, pink aerial mycelium (S. ruber and $S$. fradiae). Finally, a group has been added because of its universal distribution, easy recognition, and the fact that it includes the type species for the genus (S. albus).

\section{Streptomyces antibioticus Group}

This group was first recognized as a common and important group of soil actinomycetes in 1941. It comprises a number of strains capable of producing the antibiotic actinomycin, as well as a variety of nonactinomycin-producing strains.

Species and cultures belonging to this group are characterized by the production of a cream-colored growth, the color gradually turning brown. The aerial mycelium is gray to dark gray, sometimes shaded yellowish or even green. The soluble pigment in organic media is black to purplish. The aerial mycelium is characterized by the production of straight spore-bearing hyphae, arranged in clusters or broom-shaped bodies. The capacity to produce aerial mycelium may be lost upon continued cultivation of the cultures.

This group comprises a number of species, notably $S$. antibioticus, $S$. griseolus, $S$. fasciculus, and a number of other cultures that appear to be synonyms of these.

\section{Streptomyces lavendulae Group}

This is the chromogenic group. The ability of certain cultures of actinomycetes to form soluble dark brown pigments in complex organic media was first recognized by Rossi-Doria and Gasperini in 1891. Typical cultures were described under the name Streptothrix chromogena or Actinomyces chromogenus.

The group is widely distributed in the soil and is represented by numerous types, which have been given a variety of names. The typical growth is cream-colored to brownish. The aerial mycelium is well developed; it is cottony in nature, at first white to light gray, becoming lavender or vinous lavender. The sporophores usually form spirals. The spores are spherical to oval, $0.5-0.8 \mu$ in diameter. The cultures are strongly proteolytic and diastatic. They are characterized by production of brown to black soluble 
pigment in protein-containing media. Many of the strains are strongly antagonistic and are capable of producing various antibiotics.

This group com rises a number of species, of which the most important, from the point of view of antibiotic production, are $S$. lavendulae and $S$. venezuelae, the organisms that form streptothricin and chloramphenicol, respectively. A number of other species have been described and numerous cultures isolated capable of producing various antibiotics related to streptothricin, including antibiotic 136, streptolin, and actinorubin. These cultures appear to belong to the $S$. lavendulae group.

Streptomyces lavendulae comprises numerous cultures extremely variable in nature. Many of these cultures give rise, on cultivation, to different mutants or variants. Some of these variants produce on glucose-peptone agar a blue diffusible pigment; others form a brown pigment. The vegetative mycelium of the blue pigment-forming variants is pale blue with scattered, small pinpoint areas of deep blue. Upon complete sporulation, the vegetative growth becomes covered with the characteristic cottony lavendercolored aerial mycelium; occasional sunken areas have a slightly bluish tinge; the reverse of the vegetative growth is cream-colored except for the small blue spots. Other variants produce a colorless to cream-colored vegetative growth free of any blue pigment whatsoever; a brown diffusible pigment appears later, and the growth becomes covered with thick lavendercolored mycelium. The two types of variants are stable in nature. Other variants may lose the capacity to produce aerial mycelium.

Streptomyces venezuelae, as well, produces a number of variants. Two strains were isolated and found to be similar in their cultural and physiological properties to $S$. lavendulae, although they differed in their ability to utilize various carbohydrates. The $S$. venezuelae strains utilized arabinose, rhamnose, xylose, lactose, and fructose; S. lavendulae either had no effect or only a limited effect on these carbohydrates. The former also differed from the latter in their sensitivity to actinophage and in various serological reactions.

S. venezuelae was described as having vegetative mycelium thin-walled, colorless, hyaline, monopodially branched, the hyphae varying in diameter from 0.9 to $1.8 \mu$ and the branches growing to about $150 \mu$ in length. The aerial mycelium is lavender under the microscope, thick-walled, generally not much branched, straight or slightly and irregularly curved, not forming spirals, individual hyphae arising frequently from the primary mycelium at the surface of the substrate. The color of colonies when viewed on agar without magnification is gray to light tan or pink, but not lavender. The upper portions of the aerial hyphae divide into chains of spores. These are oval to oblong, $0.4-0.9$ by $0.7-1.6 \mu$. Individual spores are colorless at 
maturity but in mass appear tan to gray when viewed without magnification.

\section{Streptomyces griseus Group}

S. griseus was first isolated and described by Krainsky in Russia, in 1914. Soon afterward, a similar culture was isolated in the United States and described, in 1916, by Waksman and Curtis. Although they designated their culture as $S$. griseus Krainsky, there are certain marked differences bet een the two descriptions. Since no type culture was available for comparison, the two isolates may be considered as distinct.

S. griseus Krainsky produced a light gray to dark gray aerial mycelium; the sporophores were spiral-forming, giving rise to tight spirals; growth on potato was colorless, with a brown soluble pigment. On the other hand, S. griseus Waksman and Curtis produced a water-green to yellowish green aerial mycelium; the sporophores were straight and produced in tuft-like masses; growth on potato was yellowish, wrinkled, without any soluble pigment. Since the streptomycin-producing culture isolated in 1943 is identical with the one described by Waksman and Curtis, it must be considered in the light of that description. The same is true of the grisein, candicidin, and other antibiotic-producing strains of S. griseus. There are certain minor differences, however, in the cultural properties of the various strains.

The important characteristics of $S$. griseus are, therefore, formation of straight chains of spherical or oval spores, no spiral formation, rapid liquefaction of gelatin, and coagulation of blood serum.

Streptomyces griseus represents an extremely variable group of organisms. This is true particularly when the cultures are examined from the point of view of their ability to produce antibiotic substances, as pointed out previously. On this basis, the many cultures isolated and studied in detail can be classified into five distinct groups:

1. Strains which produce streptomycin. The amount of antibiotic produced varies greatly with the individual strain, under different conditions of culture. These strains are as a rule sensitive to actinophage.

2. Strains which produce grisein or grisein-like substances. These strains are as a rule resistant to actinophage.

3. Strains which produce the antifungal agent eandicidin and produce no antibacterial substance.

4. Strains which produce other antibiotics active against gram-positive bacteria only. The exact nature of these antibiotics is still unknown.

5. Strains which produce no antibiotics at all.

The streptomycin-producing strains of $S$. griseus give rise readily to mutants. So far, two mutants have been isolated: (a) a colorless form, pro- 
ducing no aerial mycelium, forming no streptomycin, and sensitive to this antibiotic; (b) a pigmented mutant, forming pink to vinaceous-colored vegetative growth, but producing the typical aerial mycelium; this mutant forms no streptomycin but gives rise to an antibiotic (rhodomycetin), which is not active against gram-negative bacteria.

Various strains of $S$. griseus produce at least two other antibiotics, one of which, cycloheximide, is active only against fungi, and another, streptocin, which is active against certain protozoan-like organisms. Streptocin is present in limited amount in the culture filtrate of the organism and more abundantly in the mycelium; it is soluble in organic solvents and is not active against gram-negative bacteria.

Bennett and Lindenfelser demonstrated that a majority of streptomycinproducing strains of $S$. griseus form a green soluble pigment in calcium malate medium and a yellow pigment in calcium succinate medium. On the other hand, grisein-producing and nonstreptomycin-producing strains of this organism do not form any green or yellow pigments in these media, although they show the typical greenish pigmentation of the aerial mycelium.

Okami examined 47 strains of streptomycin-producing cultures, 5 griseinproducing strains, and 4 pink-pigment producers. He divided them into three categories:

1. The typical $S$. griseus cultures.

2. Pink-pigment-producing forms.

3. Those that do not produce any yellow pigment when grown on potato plug.

The streptomycin-producing strains grew in maltose-containing media with $\mathrm{NaNO}_{3}$ as a source of nitrogen, but not in glucose, glycerol, or sucrose media. The grisein-producing strains grew in media containing the four compounds. The pink-pigment strains grew only in glycerol media. The utilization of the carbohydrate depends largely on the nitrogen source. In the presence of ammonium sulfate, the above differences disappeared. All strains utilized xylose, but not raffinose and rhamnose. Sensitivity to phage was said to be strain specific, but not characteristic of streptomycin production. The use of streptomycin-resistant and -dependent strains is supplementary to the foregoing differentiation methods.

\section{Streptomyces flavus Group}

The $S$. flavus group represents an extremely abundant group of organisms, the individual members varying greatly in some of their cultural properties on artificial media. This group has been known since 1891, when RossiDoria described a culture under the name of Streptothrix albido-flava. Another culture was soon described by Gasperini, in 1892, as Actinomyces 
albido-flanus. Numerous other cultures belonging to this group have been isolated from soil and other natural substrates by Krainsky, by Waksman and Curtis, and by others.

This group is characterized by cream-colored to yellow to golden yellow growth on most media. The aerial mycelium is white to gray to mouse-gray. The sporophores are long, spiral-shaped; the spores are spherical, usually $0.7 \mu$ in diameter. No brown pigment is produced on protein media. A yellowish green to golden pigment is often formed in synthetic and complex organic media. The cultures are strongly proteolytic and diastatic; sucrose is inverted, nitrate is reduced. Many of the strains are strongly antagonistic and are able to produce active antibiotics, some of which have found extensive application as chemotherapeutic agents.

The following species have been recognized and described in the literature. Various others have been listed but only insufficiently described. The recognized species can be classified as follows:

A. Do not excrete any soluble pigment into medium.

I. Colonies lemon-yellow to golden yellow.

a. Aerial mycelium not well developed.

a. Proteolytic activity strong.

Streptomyces citreus

b. Proteolytic activity weak.

Streptomyces alboflavus

2. Aerial mycelium well developed.

Streptomyces flavus

II. Colonies yellowish brown to bright yellow.

1. Colonies bright yellow, golden.

a. Proteolytic activity strong.

Streptomyces sulfureus

b. Proteolytic activity weak.

Streptomyces aureus

$S$. antibioticus and $S$. tanashiensis probably also belong to this group.

2. Colonies yellow-brown.

a. Cultures do not excrete any brown substances into medium. $a^{1}$. Cultures decompose cellulose.

Streptomyces cellulosae

$\mathrm{b}^{1}$. Cultures do not decompose cellulose.

Streptomyces graminearis

b. Cultures excrete brown substance into medium.

$a^{1}$. Aerial mycelium green.

$a^{2}$. Thermophilic forms.

$a^{3}$. Aerial mycelium dark gray or green.

Streptomyces thermophilus 
$b^{8}$. Aerial mycelium brown or chocolate.

Streptomyces thermofuscus

$b^{2}$. Mesophilic form.

Streptomyces flavochromogenes

$b^{1}$. Aerial mycelium white or light gray.

$a^{2}$. Nitrate reduced to free nitrogen.

Streptomyces denitrificans

$b^{2}$. Nitrate reduced to nitrite.

Streptomyces olivaceus

B. Excrete a soluble pigment into medium.

1. Pigment brown on organic media; cultures absorb moisture from air and condense it on aerial mycelium.

Streptomyces hygroscopicus

2. Cultures do not absorb moisture from air, pigment golden yellow.

a. Weakly proteolytic, nonantagonistic.

Streptomyces flaveolus

b. Strongly proteolytic, strongly antagonistic.

$a^{1}$. Aerial mycelium white, becoming brownish gray to dark drab-gray.

\section{Streptomyces aureofaciens}

$b^{1}$. Aerial mycelium between white and pallid quaker drab. $a^{2}$. Spirals formed.

Streptomyces rimosus

$b^{2}$. Spirals not formed.

Streptomyces griseoflavus

Among the numerous other cultures that could be included in this group, it is sufficient to list the following described in the literature: S. krainskii, S. setonii, S. microflavus, S. flavoviridis, and S. longisporoflavus.

\section{Streptomyces ruber Group}

Members of this group have been known since 1889, when Macé described an organism under the name of Cladothrix rubra. Numerous other cultures were later isolated and described, under different names, by Krainsky, Waksman and Curtis, Krassilnikov, and many others.

The group is characterized by bright red, red-orange, or rose-red vegetative growth, depending on the composition of the medium and conditions of growth. The cultures may show considerable variation in pigmentation of the vegetative growth, from purple-red to light rose. The pigment is usually not excreted into the medium, unless the latter contains fatty substances in which the pigment is soluble. The aerial mycelium is not well 
developed; it is usually produced on synthetic media as a thin, rose-white cover, or it is formed only in isolated sectors or spots. The sporophores are straight or spiral-shaped; the spores are spherical to oval, $0.7-0.8$ by 0.8 $1.0 \mu$.

The cultures are not very strongly proteolytic or diastatic. Sucrose is readily inverted. Some of the species belonging to this group are active antibiotic-producers.

Although a number of species are listed in the literature, only a few have been carefully described. These can be briefly classified as follows:

A. Colony red to orange-red to dark red.

I. Proteolytic activity strong.

II. Proteolytic activity weak.

Streptomyces bobiliae

1. Vegetative growth bright red to red-orange; aerial mycelium poorly developed.

a. Cultures lyse readily.

Streptomyces ruber

b. Chlamydospores produced abundantly in substrate mycelium.

Streptomyces aurantiacus

2. Vegetative growth at first yellowish, then red; aerial mycelium well developed. . Streptomyces erythreus

B. Colony light red or rose.

I. Proteolytic activity weak.

II. Proteolytic activity strong.

Streptomyces roseus

1. Does not hydrolyze starch.

Streptomyces albosporeus

2. Hydrolyzes starch readily.

Streptomyces roseodiastaticus

\section{Streptomyces fradiae Group}

This group is characterized by a colorless to yellowish to orange-colored growth on various media. A well-developed aerial mycelium is produced; it is colored rose to pink (seashell pink) to light orange. No soluble pigment is produced on synthetic or complex organic media. The cultures do not reduce nitrate. They are strongly antagonistic and strongly proteolytic. Some of the strains do not form any spirals in the aerial mycelium, others do.

Of the two neomycin-producing cultures, 3535 and 3554, the first does not form any spirals and thus agrees with the original description by Waksman and Curtis. The second produces some spirals of the closed type. There are also differences in pigmentation of the aerial mycelium on synthetic media. 
A number of strains belonging to this group have been isolated. Some of them produce active antibiotics. Several other species described in the literature appear to be related to this group. Here belong S. roseus, $S$. roseoflavus, and others.

\section{Streptomyces albus Group}

This group comprises a large number of organisms, characterized by the production of colorless growth on artificial media; the growth is leathery and compact. White aerial mycelium is formed on most media. The sporophores are long and produce spirals; the spores are spherical. The various cultures grow well on complex organic and synthetic media. They are not very strongly proteolytic or diastatic. They do not contain any strongly antibiotic-producing forms, although the first preparation possessing antibacterial properties ever recorded for an actinomyces was obtained from a member of this group, as shown by Gratia and Dath in 1925; this preparation, actinomycetin, has never obtained any great importance as an antibiotic agent. This group is widely distributed in nature, especially in soil and in dust.

Cultures appear to produce active hydrolytic enzymes. Various systems of classification have been proposed for this group. It is to be remembered that at one time all actinomycetes were classified into two groups, (a) $A$. albus, comprising those that produce an aerial mycelium, and (b) $A$. chromogenus, comprising those that form a black pigment on organic media. Duche appeared to follow this system, since he designated his whole monograph as A. albus.

Among the other classifications of this group, the one suggested by Krassilnikov is most logical. The separation of the species is based upon the intensity of pigmentation, growth temperatures, odor production, and certain other properties. A typical system is presented here:

A. Aerial mycelium whitish or light gray, but not snow-white.

I. Aerial mycelium produced uniformly over entire vegetative growth. 1. Cultures are able to grow in or to withstand high temperatures. a. Thermophilic

b. Thermotolerant

Streptomyces thermodiastaticus

Streptomyces casei

2. Cultures do not withstand high temperatures.

a. Strongly proteolytic.

$a^{1}$. Decompose proteins with the formation of $\mathrm{H}_{2} \mathrm{~S}$ and $\mathrm{NH}_{3}$

Streptomyces putrificans

$b^{1}$. Do not form any bad-smelling products.

Streptomyces gelaticus 
b. Weakly proteolytic.

$a^{1}$. Produce a brown substance on synthetic media.

\section{Streptomyces californicus}

$b^{1}$. Do not produce any brown substance on synthetic media. $a^{2}$. Grow at an acid reaction.

Streptomyces acidophilus

$b^{2}$. Grow at a neutral reaction.

Streptomyces albidus

II. Aerial mycelium produced in concentric rings.

Streptomyces annulatus

B. Aerial mycelium yellowish.

\section{Streptomyces albidoflavus}

Various other species belonging to the genus Streptomyces can be classified into groups, on the basis of their morphology (whorl-producers), physiology (pigment-producers), activity (pathogenicity), or utilization (antibioticproducers). It is sufficient to mention particularly the very large Streptomyces scabies group, comprising cultures or species producing potato scab; the Streptomyces coelicolor group, producing red and blue or violet pigments on synthetic media; the Streptomyces viridis group, producing green pigments; the Streptomyces globisporus group.

\section{The Genus Nocardia}

Nocardias represent a group of organisms which include both parasites and saprophytes. Their relationship to the mycobacteria, on the one hand, and to the streptomycetes, on the other, has already been indicated. The fact that a culture has been isolated from a lesion in man or animal is no proof that it is the causative agent of the particular infection; it may be a secondary agent or a member of a mixed infection.

Several systems of classification have been suggested for this group of organisms. Of these, one by Krassilnikov and the other by Waksman and Henrici are considered here in detail.

Since nocardias occupy, so far, only a secondary place as producers of antibiotics, no detailed descriptions of groups are presented.

\section{Classification System of Krassilnikov}

A. Cultures colorless, some excreting a brown substance into the medium.

I. Aerial mycelium and spore-bearing hyphae produced in culture media.

1. Substrate and aerial mycelium occasionally forming septae; the hyphae break up into long rods, $15-30 \mu$; spherical bodies not formed.

a. Saprophytes, found on dead substrate. Nocardia actinoides 
b. Parasites, living in bodies of man and animals.

Nocardia gedanensis

2. Mycelium producing frequent septae; hyphae break up into short rods and cocci.

a. Saprophytes.

$a^{1}$. Grow on protein media.

Nocardia actinomorphus

$b^{1}$. Grow on paraffin.

Nocardia paraffinae

b. Parasites.... Nocardia bovis

II. Cultures produce aerial sporophores on surface of colonies, but no aerial mycelium; sporophores are short, straight, covering the surface of the colonies with a thin pale cover.

1. Cultures grow in organic media.

Nocardia albicans

2. Cultures grow in inorganic media.

Nocardia oligocarbophilus

III. Cultures not forming any sporophores or any aerial mycelium; colonies smooth or lichnoid.

1. Saprophytes living on dead substrate.

Nocardia albus

2. Parasites or symbionts living within plants, animals, or man.

a. Organisms living in symbiosis with plants, forming nodules on their roots.

Nocardia alni

Nocardia myricae

Nocardia elaeagnii

b. Organisms living in the bodies of man and animals.

$\mathrm{a}^{1}$. Anaerobes, living in absence of oxygen.

Nocardia anaerobicus

$b^{1}$. Aerobes, or microaerophilic forms.

$a^{2}$. Strict aerobes.

Nocardia lignieresi

$b^{2}$. Facultative aerobes.

$a^{3}$. Cells non-acid-fast.

Nocardia israeli

$b^{3}$. Cells acid-fast.

Nocardia muris

B. Cultures pigmented.

I. Cultures pigmented violet or blue, the pigments diffusing into the substrate. 
1. Well-developed substrate mycelium produced, hyphae forming occasional septae and breaking up into long rods, 20-30 $\mu$; colonies form a faint aerial mycelium with straight sporophores; spores cylindrical.

Nocardia gabritschewski

2. No aerial mycelium produced, hyphae or substrate mycelium forming frequent septae and breaking up into short rods and cocci . . . . . . . Nocardia cyaneus

II. Red or orange pigment produced.

1. Mycelium forming occasional septae and breaking up into long rods; some give rise to a faint aerial mycelium and short straight sporophores.

a. Saprophytes.

$a^{1}$. Cultures not forming any soluble pigment in medium.

Nocardia fructiferi

$\mathrm{b}^{1}$. Cultures producing a brown substance.

Nocardia polychromogenes

b. Parasites living in bodies of man and animals.

Nocardia freeri

2. Hyphae forming frequent septae and breaking up into short rods and cocci; no aerial mycelium produced.

a. Saprophytes living on dead substrates.

Nocardia ruber

b. Parasites living in bodies of man and animals.

$a^{1}$. Cells acid-fast

Nocardia asteroides

$b^{1}$. Cells non-acid-fast.

Nocardia variabilis

III. Cultures citron-yellow or bright yellow.

1. Faint aerial mycelium with straight sporophores and cylindrical spores produced.

a. Saprophytes.Nocardia flavescens

b. Parasites.... Nocardia somaliensis

2. No aerial mycelium produced.

a. Saprophytes.

$a^{1}$. Cultures yellow or bright yellow.

Nocardia flavus

$b^{1}$. Cultures citron-yellow.

Nocardia citreus

b. Parasites. 
$a^{1}$. Cells acid-fast.

Nocardia farcinica

$b^{1}$. Cells non-acid-fast.

Nocardia putoriae

IV. Cultures pigmented green.

1. Saprophytes... Nocardia viridis

2. Parasites......Nocardia pyogenes

V. Cultures black.

1. Saprophytes. . Nocardia niger

2. Parasites......Nocardia sendaiensis

\section{Classification System of Waksman and Henrici}

A. Partially acid-fast organisms with strongly refractive cells; nonproteolytic and generally nondiastatic; capable of utilizing paraffin.

I. Initial mycelium fully developed, well-branching, dividing into rods and generally into cocci.

1. Vegetative mycelium soft, without macroscopically visible aerial mycelium.

a. Vegetative mycelium yellow, orange, or red.

$a^{1}$. Pathogenic.

$a^{2}$. Vegetative mycelium white, buff, or pale yellow.

1. Nocardia farcinica

$\mathrm{b}^{2}$. Vegetative mycelium yellow to red.

2. Nocardia asteroides

$b^{1}$. Not pathogenic.

3. Nocardia polychromogenes

b. Vegetative mycelium white to pink.

$a^{1}$. Gelatin not liquefied.

$a^{2}$. Growth on nutrient agar opaque, cream-colored; coccoid forms in broth.

4. Nocardia opaca

$b^{2}$. Growth on nutrient agar watery; no coccoid forms in broth.

5. Nocardia erythropolis

$c^{2}$. Growth on nutrient agar pink.

$a^{3}$. Aerial mycelium on milk white.

6. Nocardia leishmanii

$b^{3}$. Pellicle on milk pink.

7. Nocardia caprae

$c^{3}$. Pellicle on milk yellow.

8. Nocardia pretoriana 
$d^{3}$. Causing galls on blueberry plants.

$b^{1}$. Gelatin liquefied.

9. Nocardia vacciniae

10. Nocardia pulmonalis

2. Vegetative mycelium hard, yellow, with white aerial mycelium; hyphae divide into chains of acid-fast cocci.

11. Nocardia paraffinae

II. Initial mycelium very short, rapidly dividing into rods and cocci. 1. Slowly growing organisms; cells 0.5 to $0.7 \mu$ in diameter.

12. Nocardia minima

2. Rapidly growing organisms; cells 1.0 to $1.2 \mu$ in diameter.

a. Growth pink.

$a^{1}$. No cystites (swollen cells) formed.

$a^{2}$. No indigotin from indole.

13. Nocardia corallina

$\mathrm{b}^{2}$. Indigotin from indole.

14. Nocardia globerula

$b^{1}$. Cystites formed.

15. Nocardia salmonicolor

b. Growth coral-red.

16. Nocardia rubropertincta

c. Growth dark red.

17. Nocardia rubra

d. Growth white.

$a^{1}$. No aerial mycelium.

18. Nocardia coeliaca

$b^{1}$. Aerial mycelium.

19. Nocardia transvalensis

B. Non-acid-fast organisms with weakly refractive cells; no distinct formation of cocci; diastatic.

I. Nonproteolytic.

1. Growth on agar pale cream.

20. Nocardia mesenterica

2. Growth on agar whitish.

21. Nocardia albicans

3. Growth on agar yellow.

22. Nocardia flava

4. Growth on agar green.

23. Nocardia viridis

5. Growth on agar yellow-green.

24. Nocardia citrea 
6. Growth on agar pink to crimson.

25. Nocardia madurae

7. Growth on agar dark brown and even black.

26. Nocardia nigra

8. Growth consistency soft; aerial mycelium sparse.

27. Nocardia lutea

9. Growth consistency medium; aerial mycelium profuse.

28. Nocardia blackwellii

10. Good action on milk; growth consistency firm; aerial mycelium liberal.

29. Nocardia cuniculi

11. Pigment on protein media deep brown.

30. Nocardia rangoonensis

12. Pigment on protein media light brown.

31. Nocardia caviae

II. Proteolytic.

1. Growth on nutrient agar with rapid formation of unbranched diphtheroid-like rods; no typical cystites; broth turbid.

32. Nocardia actinomorpha

2. Growth white, shiny or pale, dough-like consistency; breaks up into short rods.

33. Nocardia alba

3. Growth on nutrient agar with extensive mycelium; simple unbranched rods not formed; cystites present. Broth clear.

4. Growth cream-colored.

34. Nocardia flavescens

35. Nocardia gibsonii

5. Growth rose-colored to bright red or red-orange.

36. Nocardia fructifera

6. Growth pink.

a. Gelatin not liquefied.

37. Nocardia africana

b. Gelatin liquefied, at first slowly, then completely.

38. Nocardia pelletieri

7. Colonies orange-yellow to orange-red, which may change to black.

39. Nocardia maculata

8. Pigment on protein media light brown.

40. Nocardia rhodnii

9. Pigment on protein media green to greenish brown.

41. Nocardia gardneri 
10. Growth yellowish to golden brown.

42. Nocardia fordii

11. Growth yellow to reddish brown; soluble pigment brown to red. 43. Nocardia kuroishi

\section{The Genus Micromonospora}

The genus Micromonospora is characterized by the production in nutrient media of a well-developed vegetative mycelium, $0.3-0.6 \mu$ in diameter, partly penetrating into the medium. The vegetative hyphae are straight or curved, branching, without cross walls. Aerial mycelium is not produced at all or only in rudimentary, nonsporulating form, the hyphae arising upward from vegetative mycelium.

Multiplication takes place by means of fragments of mycelium and special conidia produced singly, at the ends of sporophores arising upward from substrate mycelium. The sporophore produces a swelling at the end, and later the swelling is separated by a cross wall giving rise to spherical, oval, or oblong conidia $1.0-1.5$ by $0.8-1.2 \mu$. The conidiophores are often branched, each branch forming a conidium at the end, giving rise to a grape-like bunch of conidia. The conidia germinate in a manner similar to the spores of Streptomyces. The mycelium and conidia are gram-positive, non-acid-fast.

The colonies are similar to those of Streptomyces. They are compact, leathery, smooth or lichnoid, raised or flat. They are frequently colored red or orange or yellow, occasionally brown or green to almost black or blue. The pigments, except the dark brown, are not dissolved into the medium.

They are aerobic and grow readily at $35-37^{\circ} \mathrm{C}$.

They utilize various carbon and nitrogen sources, both organic and inorganic.

Two systems of classification of this genus are presented here.

\section{Classification System of Krassilnikov}

A. Sporophores long, branched.

I. Spores spherical.

1. Spores singular, or sporophores with little branching.

\section{Micromonospora chalceae}

2. Spores produced on numerous branches.

a. Cultures colorless; spores appear in mass as brown-pigmented..... Micromonospora globosa

b. Cultures pigmented.

$\mathrm{a}^{1}$. Cultures dark green; spores blue.

Micromonospora coerulea 
$b^{1}$. Cultures green; spores black or brown.

Micromonospora bicolor

$c^{1}$. Pigment not excreted into substrate.

Micromonospora parva

$d^{1}$. Red-brown pigment excreted into substrate, spores dark brown.

B. Sporophores short, branched.

\section{Micromonospora fusca}

I. Spores elongated. .Micromonospora monospora

II. Spores oval or spherical.

1. Cultures colorless; spores in mass white.

Micromonospora vulgaris

2. Cultures brown; spores dark brown.

Micromonospora elongata

Classification System of Jensen (emend.)

A. Vigorously growing organisms, with copious spore formation.

I. Vegetative mycelium pale pink to deep orange; no typical soluble pigment.

\section{Micromonospora chalcea}

II. Vegetative mycelium orange, changing to brownish black; soluble pigment brown.

\section{Micromonospora fusca}

B. Slowly and feebly growing organisms, with scant spore formation on glucose-asparagine agar; no soluble pigment.

I. Vegetative mycelium pale pink to pale orange.

3. Micromonospora parva

II. Vegetative mycelium yellow to orange-red.

4. Micromonospora globosa

III. Vegetative mycelium blue.

5. Micromonospora coerulea.

\section{The Genus Thermoactinomyces}

The genus Thermoactinomyces is in some respects similar to Micromonospora, especially in its ability to produce single conidia at the tips of simple or branching conidiophores, which may be so short that they often appear to be produced directly on the mycelium. In other respects, notably in appearance and in its ability to produce a true aerial mycelium, this genus resembles Streptomyces. It comprises so far only thermophilic forms capable of growing at $50^{\circ}-65^{\circ} \mathrm{C}$. Some have their optimum at $60^{\circ} \mathrm{C}$. Three species have so far been recognized. 
A. Aerial mycelium white; no soluble pigment.

B. Aerial mycelium grayish green.

Thermoactinomyces vulgaris

Thermoactinomyces monospora

C. Aerial mycelium white; soluble wine-colored to rose pigment in certain media.............Thermoactinomyces thalpophilus

\section{The Genus Actinomyces}

Although no antibiotics have so far been demonstrated for members of the genus Actinomyces, this group is included to make the guide to the actinomycetes complete.

The genus Actinomyces comprises anaerobic or microaerophilic organisms; mostly parasitic. Nonparasitic forms have been insufficiently studied. The species are non-acid-fast, nonproteolytic, and nondiastatic.

1. Colonies soft, smooth, uniform, not adherent to medium; no aerial hyphae. . Actinomyces bovis

2. Colonies tougher in texture and warted in appearance, adherent to medium; scanty aerial growth of hyphae.

Actinomyces israeli. 


\section{Description of Species of Streptomyces}

To make this guide complete, detailed descriptions are included of the more important and officially recognized species of the genera Streptomyces, Nocardia, and Micromonospora. Most of these have been isolated from soils, composts, peats, and water basins; some have come from dust and food materials. Those forms which were isolated from plant disease lesions may or may not be the causative agents of such diseases; they certainly should be considered on a par with the soil-inhabiting forms.

The cultures of actinomycetes that were isolated from diseased animals or from human infections, especially the species of Streptomyces, cannot be considered as the causative agents of the diseases, since their pathogenic nature has, in the great majority of cases, not been established experimentally.

No detailed data are presented concerning literature references, synonyms, incompletely described species, and specific natural habits. For such information, the reader is referred to the latest edition of Bergey's Manual.

1. Streptomyces albus (Rossi Doria emend. Krainsky) Waksman and Henrici (Rossi-Doria, T., Ann. Ist. Ig. Sper. Roma N. S., 1, 1894, 399-438).

Vegetative growth: Hyphae branched, $1 \mu$ in diameter.

Aerial mycelium: Abundant, white. Hyphae 1.3-1.7 $\mu$ in diameter, forming coiled chains on lateral branches. Spores ellipsoidal.

Nutrient agar: No aerial mycelium; chalky white deposit on old colonies.

Glucose agar: Aerial mycelium gray becoming brownish.

Potato: Growth folded cream-colored. Aerial mycelium white.

Gelatin: Colonies gray, no soluble pigment. Liquefaction strong.

Milk: Cream-colored surface ring. Aerial mycelium white. Rapid peptonization of milk.

Starch: Aerial mycelium white, covering the whole surface. Rapid hydrolysis in some cultures; others show no hydrolysis.

Nutrient broth: Flaky growth on bottom with surface pellicle in old cultures; aerial mycelium white.

Nitrate: Reduction to nitrite.

Calcium malate agar: Colonies covered in center with white aerial mycelium.

Odor: Earthy or musty. 
Antagonistic properties: Usually none. Some strains produce actinomycetin. Some produce thiolutin or endomycin.

Habitat: Universal occurrence in dust and soil.

Remarks: Because of the universality of this species and the ease of its superficial identification, numerous strains with varying physiological properties have been reported in the literature.

2. Streptomyces longisporus (Krassilnikov) comb. nov. (Krassilnikov, N. A., Actinomycetales, Akad. Nauk. USSR, Moskau, 1941, 47.)

Vegetative growth: Colonies colorless. Some strains produce a brown substance in protein media.

Aerial mycelium: White. Sporophores long, with many curls, weak spirals, occasionally forming small brooms. Spores cylindrical, with sharply cut ends, $1.0-1.7$ by $0.6-0.8 \mu$; later spores may become oval, $0.9-1.0$ by $0.5-$ $0.8 \mu$.

Gelatin: Liquefaction rapid.

Milk: Coagulation and peptonization rapid.

Starch: Hydrolysis strong.

Cellulose: Growth fair to good.

Nitrate: Reduction varies.

Antagonistic properties: None.

Remarks: Distinguished from Streptomyces albus in that its spores are never spherical.

3. Streptomyces globisporus (Krassilnikov) comb. nov. (Krassilnikov, N. A., Actinomycetales, Akad. Nauk. USSR, Moskau, 1941, 48.)

Vegetative growth: Colonies flat, colorless, not pigmenting medium.

Aerial mycelium: Well-developed, powdery, white. Sporophores straight, frequently forming brooms. Spores oval and spherical, $0.8 \mu$ in diameter.

Synthetic agar: Growth good, giving flat, colorless colonies, not coloring medium. Well-developed, powdery, white with trace of yellow.

Nutrient agar: Colonies grayish, smooth or lichnoid; aerial mycelium poorly developed.

Potato: No aerial mycelium or trace; faint brown color of plug.

Gelatin: Liquefaction rapid.

Milk: Peptonization rapid; no coagulation.

Starch: Hydrolysis slow.

Cellulose: Growth good.

Sucrose: No inversion.

Antagonistic properties: None or weak.

Remarks: Several substrains of this species were recognized on the basis of milk coagulation, proteolysis, and pigmentation of aerial mycelium. 
4. Streptomyces annulatus (Beijerinck emend. Krassilnikov) comb. nov. (Krassilnikov, N. A., Actinomycetales, Akad, Nauk, USSR, Moskau, 1941, 40.)

Aerial mycelium: Whitish or light gray. Sporophores produce spirals, with 3 to 7 turns (sinistrorse); spores spherical, $0.7 \mu$.

Synthetic agar: Growth colorless, flat, penetrating deep into agar. Aerial mycelium white, velvety, growing in the form of concentric rings.

Nutrient agar: Aerial mycelium white, concentric rings less marked.

Gelatin: Liquefaction slow.

Milk: Coagulation, slow peptonization.

Starch: Hydrolysis.

Cellulose: Growth good.

Sucrose: Inversion.

Odor: Strong, earthy.

Antagonistic properties: Highly antagonistic against mycobacteria and gram-positive bacteria; some strains are active against fungi.

5. Streptomyces rochei Berger, Jampolsky, and Goldberg. (Berger, J., et al., Arch Biochem., 22, 1949, 476-478.)

Vegetative growth: On synthetic (calcium malate) and nonsynthetic (peptone-tomato paste) agars, vegetative mycelium is characteristically fine, 0.8 to $1.5 \mu$ in diameter, with short branches.

Aerial mycelium: Sporogenous hyphae $1.5 \mu$ in diameter, often, but not always, spirally twisted; spirals usually short and loose, with rarely more than 2 to 3 coils. Spores oval to elliptical, sometimes spherical, 1.3-2.8 by $0.8-1.5 \mu$.

Synthetic agar: Growth thin, colorless, covered with sandy lavender to dark gray aerial mycelium. Reverse of growth light gray, later becoming grayish yellow. No soluble pigment.

Nutrient agar: Growth cream-colored. Aerial mycelium white. No soluble pigment.

Glucose agar: Growth smooth, yellowish, covered with white to gray aerial mycelium. Yellowish soluble pigment.

Potato: Growth abundant, lichnoid, cream-colored. Aerial mycelium abundant, cottony, white to gray. Color of plug becomes reddish tan.

Gelatin $\left(18^{\circ} \mathrm{C}\right)$ : Surface cream-colored ring, covered with white aerial mycelium. Liquefaction rapid. Faint yellow soluble pigment.

Milk: Ring at surface cream-colored to brownish. Coagulation and rapid peptonization.

Starch: Growth brownish. Aerial mycelium mouse-gray. Reverse shows slight purple pigmentation in 4 days. Diastatic action strong.

Calcium malate-glycerin: Growth good, raised in center. Aerial mycelium 
gray, buff around the edges, having a fuzzy appearance. Medium is cleared directly under the growth.

Calcium citrate-glycerol broth: Growth at surface forming a thin mat of partially sporulated, discrete, grayish white colonies. From the mat, soft, round, fuzzy gray colonies drop into the medium.

Antagonistic properties: On certain complex nitrogenous media, such as those containing soybean flour or distillers' dried solubles, the organism produces a wide range of antimicrobial activity. Part of this is attributable to a specific antibiotic known as borrelidin.

Remarks: Morphologically the culture resembles some species in the Streptomyces albus group, such as Streptomyces albidoflavus, Streptomyces californicus, Streptomyces lipmanii, but it is not believed to be identical with any of them.

6. Streptomyces coelicolor (Müller) Waksman and Henrici. (Müller, R., Centrbl. Bakt. I, 46, 1908, 195).

Aerial mycelium: Straight filaments with open, dextrorse spirals. Spores oval or rod-shaped, $0.7-1.0$ by $0.8-1.5 \mu$.

Synthetic agar: Growth thin, spreading, colorless at first, becoming red, then blue. Aerial mycelium thin, powdery, white, becoming mouse-gray.

Nutrient agar: Growth good. Pigment lacking or faint blue.

Potato: Strong pigment production, sometimes greenish blue or violet, but usually sky-blue, diffusing through medium and coloring water at base of tube.

Gelatin: Growth good. No soluble pigment. Liquefaction rapid.

Milk: No change at $25^{\circ} \mathrm{C}$. At $37^{\circ} \mathrm{C}$, coagulation, with rapid peptonization.

Nutrient broth: Growth good. Cretaceous layer around edge.

Nitrate: Reduction positive.

Aspargine agar: Growth with glycerol as source of carbon, good violet to deep blue, with pigment diffusing through medium; final pH 7.0-8.0. Growth with glucose as source of carbon, poorer, red, no diffusion of pigment; final pH 6.0 to 5.0 .

Blood agar: Hemolysis on 4th day.

Temperature: Growth good at room temperature and at $37^{\circ} \mathrm{C}$.

Antagonistic properties: Some strains produce coelicolorin and mycetin.

Remarks: Müller reported no acid from carbohydrates on organic media. Conn recorded acid from glucose and lactose, and sometimes from sucrose and mannitol, on synthetic media.

The most striking characteristic of this organism is a litmus-like pigment, usually produced on potato or synthetic media, which is deep blue and water-soluble at alkaline reactions, violet around neutrality, and red (insoluble in water) at about $\mathrm{pH}$ 6.0. The primary pigment has a spectro- 
photometric curve almost identical with that of azolitmin. There are undoubtedly other pigments produced, especially by different other strains believed to be typical of Actinomyces violaceus ruber, a culture described by Waksman and Curtis.

7. Streptomyces pluricolor (Berestnev emend. Krassilnikov) comb. nov. (Krassilnikov, N. A., Actinomycetales, Akad. Nauk. USSR, Moskau, 1941, 17.)

Aerial mycelium: Well-developed, white-gray. Sporophores produce numerous spirals, with 3-5 turns (sinistrorse). Spores oval, 0.9 by $0.7 \mu$.

Synthetic agar: Growth at first pigmented red-yellow, then changing to blue or blue-green. The blue pigment dissolves into medium.

Potato: Growth and soluble pigment sharp blue.

Gelatin: Liquefaction rapid.

Milk: Peptonization without coagulation.

Starch: Hydrolysis.

Cellulose: No growth.

Nutrient broth: Green fluorescent pigment produced.

Sucrose: Inversion.

Antagonistic properties: None.

8. Streptomyces cyaneus (Krassilnikov) comb. nov. (Krassilnikov, N. A., Actinomycetales, Akad. Nauk. USSR, Moskau, 1941, 14.)

Vegetative growth: Pigmented blue, the pigment dissolving into medium, remaining blue at both acid and alkaline reactions.

Aerial mycelium: Sporophores produce open spirals (sinistrorse), with 2-3 turns in each. Spores oval, seldom spherical, $0.6-0.8$ by $0.6 \mu$.

Synthetic agar: Colonies at first smooth, becoming lumpy, leatherycompact, and covered with well-developed blue-gray aerial mycelium.

Gelatin: Liquefaction rapid, completed in 5-6 days.

Milk: Peptonization with prior coagulation.

Starch: Hydrolysis weak.

Cellulose: No growth.

Nitrate: No reduction.

Sucrose: No inversion.

Antagonistic properties: Weak.

9. Streptomyces vinaceus Mayer et al. (Mayer, R. L., Crane, C., DeBoer, C. J., Konopka, E. A., Marsh, J. S., and Eisman, P. C., XIIth Intern. Congr. Pure Appl. Chem., 1951, 283-284.)

Aerial mycelium: No spirals. Spores nearly spherical, 1.0-1.5 $\mu$. 
Synthetic agar: Growth rough, off-white with reverse purple-red; soluble blue-red pigment upon extended incubation.

Nutrient agar: Growth rough, dry, off-white; reverse blue-red; no soluble pigment. Several strains produce concentric growth rings.

Glucose agar: Growth rough, dry, off-white; reverse dark red-blue; redblue soluble pigment only on extended incubation.

Potato: Growth rough, slightly moist, off-white. No soluble pigment.

Gelatin: Growth sparse, tan-white; no soluble pigment. Ready liquefaction.

Starch: Growth rough, dry, elevated, spreading, off-white. Moderate hydrolysis.

Temperature: Optimum $22^{\circ}-28^{\circ} \mathrm{C}$.

Antagonistic properties: Produces vinactin, an antibiotic similar in many respects to viomycin.

Remarks: Soluble red-blue pigment produced in certain media, particularly in glucose-peptone broth by shake culture.

10. Streptomyces violaceus (Gasperini emend. Krassilnikov) comb. nov. (Gasperini, D., Centrbl. Bakt., 15, 1894, 684; Krassilnikov, N. A., Actinomycetales, Akad. Nauk. USSR, Moskau, 1941, 15.)

Vegetative growth: Well-developed, nonseptated; readily breaks up in old cultures. Colonies lichnoid, at first red becoming dark blue, finally purple-violet. Some cultures produce fat droplets in the colony, pigmented red or purple.

Aerial mycelium: Produced poorly or not at all; some substrates, like cellulose, paraffin, or fats favor its formation. Aerial hyphae long, straight, branching seldom, and also short-branched. Sporophores forming open spirals, sinistrorse curvatures. Spores spherical and oval.

Synthetic agar: Pigments dissolved into medium, which becomes purpleviolet to dark violet.

Gelatin: Liquefaction slow.

Milk: No coagulation; peptonization slow.

Starch: Hydrolysis weak.

Cellulose: Growth weak or none.

Nitrate: No reduction; few strains form nitrite.

Sucrose: Inversion rapid.

Temperature: Optimum $25-30^{\circ} \mathrm{C}$.

Antagonistic properties: Exerts strong antagonistic effect upon various bacteria.

11. Streptomyces verne (Waksman and Curtis) Waksman and Henrici. (Waksman, S. A., and Curtis, R. E., Soil Sci., 1, 1916, 120.) 
Growth: Filaments with close branching of the hyphae. Capacity to produce aerial mycelium lost on cultivation.

Synthetic agar: Growth abundant, spreading, lichnoid, glossy, yellowish becoming brownish.

Nutrient agar: Colonies small, grayish, with depressed center, becoming wrinkled.

Glucose agar: Growth abundant, lichnoid, center raised, gray with purplish tinge, entire.

Potato: Growth cream-colored, becoming gray, wrinkled.

Gelatin: Colonies small, cream-colored. Liquefaction rapid.

Milk: Ring pinkish brown. Coagulation and rapid peptonization.

Starch: Growth scant, restricted brownish; hydrolysis rapid.

Cellulose: Growth good.

Glucose broth: Sediment flaky.

Nitrate: Nitrites produced.

Temperature: Optimum $37^{\circ} \mathrm{C}$.

Antagonistic properties: Limited activity against some bacteria.

Remarks: Soluble green pigment produced when freshly isolated. In time, this pigment becomes brown.

12. Streptomyces viridans (Krassilnikov) comb. nov. (Krassilnikov, N. A., Actinomycetales, Akad. Nauk. USSR, Moskau, 1941, 33.)

Vegetative growth: Colonies green to brown-green.

Aerial mycelium: Dark gray, olive-colored, or gray-green velvety, covering the whole colony. Sporophores long, spiral-shaped. Spores cylindrical. Synthetic agar: Colonies green; soluble green pigment produced.

Nutrient agar: Growth brown-green; soluble brown substance produced.

Gelatin: Liquefaction rapid.

Milk: Coagulation and rapid peptonization.

Starch: Hydrolysis rapid.

Cellulose: Growth poor.

Nitrate: Reduction strong.

Sucrose: Inversion rapid.

Antagonistic properties: None; some strains are weakly active.

13. Streptomyces californicus (Waksman and Curtis) Waksman and Henrici. (Waksman, S. A., and Curtis, R. E., Soil Sci., 1, 1916, 122).

Aerial mycelium: Filaments with long, narrow, open spirals. Spores spherical to oval.

Synthetic agar: Growth spreading, vinaceous-colored. Aerial mycelium powdery, thin, light neutral gray. No soluble pigment.

Nutrient agar: Growth thin, restricted, yellowish to cream-colored. 
Glucose agar: Growth restricted, much folded, cream-colored, with sulfuryellow tinge.

Potato: Growth glossy, yellow to red, turning red-brown.

Gelatin: Surface growth gray, moist, abundant; no soluble pigment. Medium liquefaction.

Milk: Surface growth faint, brownish. Coagulation and slow peptonization.

Starch: Growth spreading, pink center with colorless to gray margin. Hydrolysis.

Glucose broth: Mass on surface solid cream-colored, with pink tinge.

Nitrate: Reduction to nitrite.

Temperature: Optimum $37^{\circ} \mathrm{C}$.

Antagonistic properties: Limited. Some strains produce viomycin.

14. Streptomyces virgatus (Krassilnikov) comb. nov. (Krassilnikov, N. A., Actinomycetales, Akad. Nauk. USSR. Moskau, 1941, 32.)

Vegetative growth: Colonies yellow-green to citron-yellow or pure yellow; on some media pale green. Pigment insoluble in substrate. Some strains produce brown substance in protein media.

Aerial mycelium: Weakly developed, white or pale yellow. Sporophores produced in form of tufts. Oidiospores cylindrical, elongated; in some strains round-oval.

Gelatin: Liquefaction rapid.

Milk: Coagulation and peptonization rapid.

Starch: Hydrolysis rapid.

Cellulose: No growth.

Nitrate: Reduction good.

Antagonistic properties: None.

15. Streptomyces flaveolus (Waksman) Waksman and Henrici. Waksman, S. A., Soil Sci., 8, 1919, 134).

Aerial mycelium: Numerous closed and open spirals on all media. Spores oval to elliptical.

Synthetic agar: Growth light sulfur-yellow turning to cadmium-yellow, penetrating deep into medium. Aerial mycelium white to ash-gray.

Nutrient agar: Growth white, glistening, wrinkled.

Glucose agar: Growth restricted, surface folded, raised.

Potato: Growth abundant, wrinkled, cream-colored.

Gelatin: Abundant, yellowish, spreading surface. Liquefaction rapid.

Milk: Ring sulfur-yellow. Coagulation and peptonization.

Starch: Growth white, spreading. Hydrolysis.

Glucose broth: Pellicle thin, yellow. 
Nitrate: Reduction to nitrite.

Antrgonistic properties: Some strains produce actinomycin.

Remarks: Culture produces soluble empire-yellow pigment.

16. Streptomyces parvus (Krainsky) Waksman and Henrici. (Krainsky, A., Centrbl. Bakt. II, 41, 1914, 639-688.)

Vegetative growth: Golden yellow to brick-red, depending on composition of medium.

Aerial mycelium: Poorly developed, rose-white. Sporophores produce spirals. Spores spherical to oval, $0.9-1.3$ by $1.2-1.8 \mu$.

Glucose agar: Colonies small, yellow, with aerial mycelium light yellow.

Gelatin: Colonies yellow. Liquefaction medium.

Milk: Coagulation and peptonization rapid.

Starch: Diastatic action good.

Cellulose: Growth good.

Glucose broth: Hemispherical colonies in bottom of tube.

Nitrate: Reduction slight.

Calcium malate agar: Same as on glucose agar.

Antagonistic properties: Produces actinomycin.

17. Streptomyces xanthophaeus Lindenbein (Lindenbein, W., Arch. Mikrobiol., 17, 1952, 361-383.)

Synthetic agar: Growth good, crumb-like, smooth, brownish. Aerial mycelium cottony white, later grayish or even reddish gray. Soluble pigment light brown, later yellow-brown.

Nutrient agar: Growth good, diffuse, smooth, light brown reverse. Aerial mycelium powdery ash-gray to white. Soluble pigment yellow to yellowbrown.

Glucose agar: Growth good, diffusive, colorless, with light yellow reverse. Aerial mycelium powdery, seldom velvety, abundant, light brown. Soluble pigment yellow.

Potato: Growth good, lichnoid. Aerial mycelium powdery gray. No soluble pigment.

Gelatin: Growth good, leathery, brown. Aerial mycelium velvety, ashgray. Soluble pigment yellow-brown. Liquefaction strong.

Milk: Growth good, lichnoid. Aerial mycelium gray to violet. Soluble pigment dark brown. Peptonization strong.

Starch: Growth good, lichnoid. Aerial mycelium velvety, violet-gray. Hydrolysis rapid.

Cellulose: No growth.

Glucose broth: Surface growth good, colorless, later producing heavy sediment. Abundant flocculent particles. Aerial mycelium cottony, grayish 
white, tending later to disappear. Soluble pigment light yellow, later orangeyellow.

Glucose-asparagine agar: Growth good, diffuse, colorless, light yellow reverse. Aerial mycelium powdery white. Soluble pigment light yellow.

Calcium malate agar: Growth very good, crumb-like, with yellowish red reverse. Aerial mycelium velvety, white-gray or reddish gray. Soluble pigment dark red-yellow.

Antagonistic properties: Produces antibiotics.

18. Streptomyces cellulosae (Krainsky) Waksman and Henrici. (Krainsky, A., Centrbl. Bakt. II, 41, 1914, 639-688.)

Vegetative growth: Yellow, producing soluble yellow pigment. No soluble brown pigment.

Aerial mycelium: Well-developed, gray to white-gray. Spores almost spherical, $1.3 \mu$ in diameter, often arranged in chains.

Nutrient agar: Aerial mycelium white.

Glucose agar: Growth abundant; aerial mycelium gray. Soluble yellow pigment.

Potato: Growth colorless; aerial mycelium gray.

Gelatin: Colonies circular, yellowish. Liquefaction.

Milk: Coagulation and peptonization rapid.

Starch: Diastatic action strong.

Cellulose: Growth good; decomposition limited.

Glucose broth: Growth coarse, flaky. Yellow pigment.

Nitrates: Reduction slight.

Esculin: Hydrolyzed.

Calcium malate agar: Colonies yellowish; aerial mycelium gray. Soluble yellow pigment.

Temperature: Optimum $30^{\circ}-35^{\circ} \mathrm{C}$.

Antagonistic properties: Positive.

19. Streptomyces rimosus Sobin, Finlay, and Kane. (U. S. Pat. $2,516,080$, July 18,1950 .)

Vegetative growth: Colonies flat, smooth, irregular edge, yellow-pigmented.

Aerial mycelium: Between white and pallid quaker drab; spirals numerous; conidia $0.6-0.7$ by $0.8-1.4 \mu$, cylindrical.

Nutrient agar: Growth poor, no aerial mycelium, faint yellowish pigment.

Glucose agar: Growth with dry, cracked surface; aerial mycelium mousegray; yellowish brown pigment.

Potato: Growth moderate, wrinkled; aerial mycelium white to dark; yellowish brown pigment. 
Gelatin: Aerial mycelium white, no soluble pigment. Liquefaction moderate.

Milk: Thick pellicle, aerial mycelium grayish white; no peptonization, no change in $\mathrm{pH}$.

Starch: Growth poor, thin; aerial mycelium limited; colonies cinnamondrab. Hydrolysis slight.

Cellulose: No decomposition.

Nitrate: Reduction strong.

Asparagine agar: Aerial mycelium white to pallid quaker-drab; faint yellow soluble pigment.

Odor: Earthy.

Antagonistic properties: Produces oxytetracycline, an amphoteric substance active against various bacteria, rickettsiae, and larger viruses; produces also rimocidin, an antifungal agent.

Remarks: Culture closely related to Streptomyces griseoflavus.

20. Streptomyces griseoflavus (Krainsky) Waksman and Henrici. (Krainsky, A., Centrbl. Bakt. II, 41, 1914, 639-688.)

Vegetative growth: Thin cream-colored, later becoming much-folded or lichnoid.

Aerial mycelium: Aerial mycelium powdery white, appearing first on drier edges of growth. Sporophores straight, abundantly branched; no curvatures, and no spirals produced.

Synthetic agar: Growth reddish brown to orange, covered with white aerial mycelium; faint yellowish soluble pigment.

Nutrient agar: Growth cream-colored, covered with white aerial mycelium; no soluble pigment.

Potato: Growth lichnoid, cream-colored to brownish, later becoming reddish brown; aerial mycelium powdery white to gray; no soluble pigment.

Gelatin: Growth cream-colored to brownish, covered with white aerial mycelium. Liquefaction slow, with faint yellowish coloration of liquefied zone.

Milk: Growth cream-colored to yellowish; aerial mycelium thin white. Peptonization rapid without previous coagulation.

Starch: Growth cream-colored with brownish center; no aerial mycelium. Hydrolysis limited.

Nitrate: Reduction to nitrite.

Yeast-glucose agar: Growth lichnoid, cream-colored to brownish; aerial mycelium white to grayish; soluble yellowish pigment.

Antagonistic properties: Strongly antagonistic; produces oxytetracycline and rimocidin. 
21. Streptomyces aureofaciens Duggar. (Duggar, B. M., Ann. N. Y. Acad. Sci., 51, 1948, 177.)

Vegetative growth: Mycelium hyaline, becoming yellow in 2-3 days; later golden tan to tawny.

Aerial mycelium: White, becoming brownish gray to dark, drab-gray in 5-7 days.

Nutrient agar: Growth characteristic, with golden yellow soluble pigment.

Potato: Soluble pigment golden yellow.

Antagonistic properties: Produces chlortetracycline, an amphoteric compound containing both nitrogen and nonionic chlorine, active against various bacteria, rickettsiae, and the larger viruses.

22. Streptomyces albidoflavus (Rossi Doria) Waksman and Henrici. (Rossi-Doria, T., Ann. Ist. Ig. Sper. Roma N. S. 1, 1894, 399-438.)

Synthetic asparagine agar: Growth rapidly covered with white aerial mycelium, later becoming whitish yellow; brown on reverse side; yellowish soluble pigment.

Peptone agar: Growth cream-colored, covered with fine white aerial mycelium; yellow soluble pigment.

Tyrosine agar: Growth fine with orange-yellow on reverse side; medium becomes yellowish to yellowish rose.

Synthetic asparagine solution: Long branching filaments, $0.6 \mu$ in diameter. Thicker aerial mycelium producing irregular spores; flaky growth dropping to bottom of tube. Surface growth becomes covered with yellowish white aerial mycelium; brownish on reverse side; soluble pigment yellowish.

Peptone solution: Growth rapid, much folded, partly covered with white mycelium on surface of medium; soluble yellow-ocher pigment.

Gelatin: Punctiform colonies with white aerial mycelium on surface of liquid; no soluble pigment; liquefaction rapid.

Milk: Growth rapid, becoming covered with whitish aerial mycelium; never fully covering the surface; no coagulation; peptonization begins slowly and is completed in 13 days, liquid becoming yellowish orange.

Starch: Growth cream-colored, rapidly covered with yellow aerial mycelium; after 20 days, growth becomes much folded; greenish on reverse side; slight amber color in medium.

Coagulated serum: Cream-colored growth of surface becoming covered with white aerial mycelium; liquefaction rapid.

Antagonistic properties: Positive.

Remarks: This strain is closely related to Streptomyces albus. 
23. Streptomyces lieskei (Duché) Waksman and Henrici. (Duché, J., Les actinomyces du groupe albus, P. Lechevalier, Paris, 1934.)

Synthetic agar: Growth cream-colored with delayed white aerial mycelium growing from the edge toward the center; mycelium later yellowish. Reverse of growth yellowish to green. Dirty yellow to yellow-green soluble pigment.

Nutrient agar: Growth cream-colored becoming covered with white aerial mycelium; yellowish soluble pigment.

Gelatin: Growth cream-colored becoming covered with white aerial mycelium; no soluble pigment. Rapid liquefaction.

Synthetic solution: Long branching filaments, $0.7 \mu$ in diameter. Aerial mycelium yellowish white; does not readily produce spores; flakes drop to the bottom of the tube.

Peptone solution: Cream-colored colonies on surface with flakes in the liquid dropping to the bottom of the tube. Liquid becomes yellowish.

Tyrosine medium: Rapid growth on surface with whitish yellow aerial mycelium; yellowish to orange-yellow soluble pigment.

Milk: Growth cream-colored; colorless on reverse side; no aerial mycelium. Peptonization without coagulation. After 20 days the whole milk becomes a clear yellowish liquid.

Coagulated serum: Growth clear-colored. Liquefaction rapid.

Remarks: Culture related to Streptomyces alboflavus and Streptomyces albidoflavus.

24. Streptomyces flavovirens (Waksman) Waksman and Henrici. (Waksman, S. A., Soil Sci., 8, 1919, 117.)

Aerial mycelium: Large masses of minute tufts; the hyphae coarse, straight, short, relatively unbranched, beaded; open spirals may be produced in certain substrates. Conidia spherical, oval to rod-shaped, 0.751.0 by $1.0-1.5 \mu$.

Synthetic agar: Growth spreading deep into the substratum, yellowish with greenish tinge. Aerial mycelium gray, powdery.

Nutrient agar: Growth yellowish; the reverse dark in center with yellowish zone and outer white zone.

Glucose agar: Growth restricted, developing only to a very small extent into the medium, yellow, turning black, edge entire.

Potato: Growth sulfur-yellow, wrinkled.

Gelatin: Yellowish green surface pellicle, consisting of a mass of small colonies, on the liquefied medium.

Milk: Cream-colored to brownish ring; coagulation; peptonization, becoming faintly alkaline. 
Starch: Growth greenish yellow, spreading, developing deep into the medium. Hydrolysis.

Glucose broth: Thick, sulfur-yellow pellicle or ring.

Nitrate: Reduction to nitrite very slight.

Temperature: Optimum $25^{\circ} \mathrm{C}$.

Antagonistic properties: Active against fungi.

Remarks: Greenish yellow soluble pigment formed.

24a. Streptomyces celluloflavus Nishimura, Kimura, and Kuroya (Nishimura, H., Kimura, T., and Kuroya, M., J. Antibiotics [Japan], 6, 1953, 57-65.)

Aerial mycelium: A few imperfect spirals. Almost spherical spores, 1.0 by $0.9 \mu$.

Synthetic agar: Glossy growth, developing deep into medium, later becoming marguerite-yellow color. Faint sulfur-yellow soluble pigment.

Calcium malate glycerol agar: Primrose-yellow growth, later turning white to pale olive-buff, with blackish center. Cottony aerial mycelium white, with grayish patches, later turning olive-buff. Citron-yellow soluble pigment.

Glucose agar: Cream to yellow-colored growth. Scant cottony white to gray aerial mycelium. Sulfur-yellow soluble pigment.

Nutrient agar: Olive-buff, turning colorless, growth. Scant, cottony, white to grayish aerial mycelium. Yellow with tinge of green, to old-gold soluble pigment.

Potato: Wrinkled, spreading, deep-olive-buff colored growth. White to olive-buff aerial mycelium. Deep olive-buff soluble pigment.

Gelatin: Ivory-yellow to olive-buff colonies on surface of liquefied portion. No aerial mycelium. Faint brownish pigment. Rapid to medium liquefaction.

Milk: Yellow to dark olive-buff growth. White aerial mycelium. Reddish brown soluble pigment. Coagulation and rapid peptonization.

Tyrosine medium: Ivory yellow to cream-buff growth. None to scant white aerial mycelium. Greenish yellow soluble pigment.

Cellulose agar: Poor growth, with yellow soluble pigment.

Antagonistic properties: Produces thiolutin.

25. Streptomyces limosus Lindenbein (Lindenbein, W., Arch. Mikrobiol., 17, 1952, 361-383.)

Vegetative growth: Crumb-like, yellowish, later becoming gray to coalblack; greenish yellow reverse.

Aerial mycelium: Powdery snow-white to gray, or completely lacking. Soluble pigment greenish yellow to citron-yellow. 
Synthetic agar: Growth good, diffuse, colorless, later becoming crumblike, light yellow. No aerial mycelium. Soluble pigment citron-yellow.

Nutrient agar: Growth diffuse, colorless; light brown in reverse. No aerial mycelium. Soluble pigment around bottom.

Glucose agar: Growth good, yellow-brown reverse. Aerial mycelium velvety white-gray to ash-gray. Soluble pigment yellow-brown.

Potato: Growth moderate, brownish yellow to light brown. Aerial mycelium powdery gray-white. Soluble pigment citron-yellow to sulfur-yellow.

Gelatin: Growth good, yellow-brown. No aerial mycelium. Soluble pigment dark brown. Liquefaction complete.

Milk: Growth good, lichnoid, light yellow. Aerial mycelium powdery gray-white. Soluble pigment light brown. Peptonization strong.

Starch: Colonies small yellowish, brownish in reverse. Aerial mycelium velvety gray-white. Soluble pigment light gray. Hydrolysis strong.

Cellulose: No growth.

Glucose broth: Growth in the form of sediment, white, later light yellow. No aerial mycelium. Soluble pigment citron-yellow.

Glucose-asparagine agar: Growth good, crumb-like, light yellow, later becoming black with citron-yellow reverse. Aerial mycelium white, later ash-gray. Soluble pigment citron-yellow.

Calcium malate agar: Growth good, crumb-like; dark yellow reverse. Aerial mycelium powdery to velvety, white, later ash-gray. Soluble pigment golden yellow.

Source: Isolated from the edge of a river.

26. Streptomyces griseoluteus Umezawa, Hayano, Maeda, Ogata, and Okami. (J. Antibiotics [Japan], 4, 1951, 34-40; 5, 1952, 477-480.)

Aerial mycelium: Hyphae branch monopodially and irregularly, conidia oval to cylindrical, $1.0-1.2$ by $1.8-2.2 \mu$.

Synthetic agar: Growth thin, colorless to cream-colored. Margin plumose, penetrating into medium. Aerial mycelium powdery, grayish white to light drab. Soluble pigment none or yellowish brown.

Nutrient agar: Growth wrinkled, transparent; aerial mycelium thin, white, powdery; soluble pigment none or yellowish brown.

Glucose agar: Growth wrinkled cream-colored. Aerial mycelium thin, white; pigment reddish brown.

Potato: Growth abundant, wrinkled, cream-colored: aerial mycelium dusty white, thin; plug becoming slightly brownish.

Gelatin: No growth.

Milk: Ring cream-colored; surface patches white.

Starch: Hydrolysis. 
Glucose broth: Surface ring cream-colored to brown. Aerial mycelium powdery white. Soluble pigment slight, reddish brown.

Nitrate: Reduction positive.

Antagonistic properties: Produces griseolutein, a yellow antibiotic active against gram-positive and gram-negative bacteria.

27. Streptomyces bobiliae (Waksman and Curtis) Waksman and Henrici. (Waksman, S. A., and Curtis, R. E., Soil Sci., 1, 1916, 121.)

Aerial mycelium: Few close spirals of a dextrorse type.

Synthetic agar: Growth abundant, glossy, wrinkled, elevated, coral-red becoming deep red. Aerial mycelium scant, white.

Nutrient agar: Growth restricted, glossy, gray, becoming brownish.

Potato: Growth thin, yellowish, becoming red, dry, and wrinkled.

Gelatin: Surface growth dense, cream-colored to brownish. Liquefaction rapid.

Milk: Ring dark brown. No coagulation. Peptonization.

Starch: Growth restricted, finely wrinkled, coral-red with hyaline margin. Hydrolysis.

Glucose broth: Round colonies in fluid. Flaky sediment.

Nitrate: Reduction to nitrites.

Temperature: Optimum $37^{\circ} \mathrm{C}$.

Antagonistic properties: Positive.

Remarks: Soluble brown pigment formed.

28. Streptomyces aurantiacus (Gasperini emend. Krassilnikov) comb. nov. (Krassilnikov, N. A., Actinomycetales, Akad. Nauk. USSR, Moskau, 1941, 36.)

Vegetative growth: Lichnoid, dry, compact. Colored bright orange or golden; color does not change into red or yellow on continued incubation. Pigment insoluble in medium, but soluble in organic solvents. Produces an abundance of chlamydospores.

Aerial mycelium: Poorly developed or completely absent on many media; nonseptated. Sporophores form spirals with 3-5 turns. Spores spherical to oval, $0.7-0.9$ by $0.6-0.8 \mu$.

Potato: Soluble pigment brown.

Gelatin: Liquefaction slow, after 20-30 days' incubation.

Milk: Peptonization weak, usually without previous coagulation.

Starch: Hydrolysis slow.

Cellulose: No growth.

Nitrate: No reduction.

Sucrose: No inversion. 
Fats: Hydrolysis and utilization rapid.

Paraffin: Growth good, with spiral-forming sporophores and spherical spores.

Antagonistic properties: Strongly antagonistic.

Remarks: This species represents a widely distributed group of organisms, including such forms as Actinomyces parvus of Krainsky and others.

29. Streptomyces griseolus (Waksman) Waksman and Henrici. (Waksman S. A., Soil Sci., 8, 1919, 121.)

Aerial mycelium: No spirals observed. Conidia spherical or oval-shaped.

Synthetic agar: Growth colorless, thin, spreading, chiefly in the medium; surface growth limited almost entirely to the aerial mycelium. Aerial mycelium at first gray, later becoming pallid, neutral-gray.

Nutrient agar: Growth brownish, with smooth surface.

Glucose agar: Growth spreading, both on the surface and into the medium; center raised, cream-colored, turning dark.

Potato: Growth cream-colored, becoming black, spreading.

Gelatin: Liquefaction, with yellowish, flaky pellicle and sediment.

Milk: Growth abundant, pink pellicle; coagulation; peptonization, becoming alkaline.

Starch: Growth grayish brown with dark ring. Hydrolysis.

Glucose broth: Ring thick, brown.

Nitrate: Reduction to nitrite.

Temperature: Optimum $25^{\circ} \mathrm{C}$.

Antagonistic properties: Considerable activity against various bacteria; some of the strains show negative activity.

Remarks: Faint brownish soluble pigment formed.

30. Streptomyces fasciculus (Krassilnikov) comb. nov. (Krassilnikov, N. A., Actinomycetales, Akad. Nauk. USSR, Moskau, 1941, 51).

Vegetative growth: Growth good, lichnoid colorless, covered with dark gray, powdery or velvety aerial mycelium.

Aerial mycelium: Sporophores straight, short, arranged in broom-shaped bodies or fascicles. Spores oblong, $1.0-1.5$ by $0.7 \mu$.

Synthetic agar: See Vegetative growth.

Nutrient agar: Aerial mycelium poorly developed, gray.

Potato: Growth heavy, folded, with well-developed, dark gray aerial mycelium. Soluble pigment brown. Sporophores and spores same as on synthetic agar.

Gelatin: Liquefaction medium.

Milk: Coagulation and peptonization ready.

Starch: Hydrolysis rapid. 
Cellulose: Growth good.

Nitrate: Reduction to nitrite poor.

Sucrose: Inversion ready.

Antagonistic properties: Strongly antagonistic.

Remarks: Related to Streptomyces candidus. Streptomyces nitrosporeus Okami (J. Antibiotics [Japan], 5, 1952, 477-480) appears to be closely related, if not identical.

31. Streptomyces erythreus (Waksman) Waksman and Henrici. (Waksman, S. A., Soil Sci., 8, 1919, 112.)

Vegetative growth: Growth spreading, with irregular margin, developing deep into the medium; color at first white, later turning yellowish, agar around growth has a white, milky surface.

Aerial mycelium: Fine, branching; numerous open spirals formed as side branches of the main hyphae.

Synthetic agar: Aerial mycelium, thick, solid, white.

Nutrient agar: Growth cream-colored.

Glucose agar: Growth abundant, spreading, cream-colored, later turning brown chiefly on surface; center raised, lobate margin.

Potato: Growth wrinkled, cream-colored, becoming yellowish.

Gelatin: growth abundant, dense, gray with pinkish tinge, chiefly on surface of liquefied portion.

Milk: Surface zone yellowish. Coagulation and peptonization.

Starch: Growth cream-colored with faint greenish tinge. Hydrolysis.

Glucose broth: Surface growth abundant, cream-colored.

Nitrate: Reduction to nitrite.

Temperature: Optimum $25^{\circ} \mathrm{C}$.

Antagonistic properties: Marked. Produces erythromycin.

32. Streptomyces flavogriseus (Duché) comb. nov. (Duché, J., Les actinomyces du groupe albus, P. Lechevalier, Paris, 1934.)

Aerial mycelium: Long, straight hyphae producing a few curling tips; spores spherical.

Synthetic agar: Growth limited, yellowish, reverse turning black; aerial mycelium thin, gray to mouse-gray.

Nutrient agar: Growth thin, cream-colored; aerial mycelium thin, white. No soluble pigment.

Glucose-peptone agar: Surface growth good, yellow; reverse tending to turn dark. Aerial mycelium abundant, mouse-gray to drab. No soluble pigment.

Potato: Growth abundant, lichnoid. Aerial mycelium abundant, mousegray to drab with white edge. No soluble pigment. 
Gelatin: Growth flocculent, through medium. Liquefaction slow. No soluble pigment.

Milk: Ring cream-colored. No aerial mycelium. Peptonization very rapid.

Starch: Growth very limited, similar to that on synthetic agar. $\mathrm{Hy}-$ drolysis.

Nutrient broth: Surface growth in clumps, cream-colored. Aerial mycelium gray.

33. Streptomyces diastaticus (Krainsky) Waksman and Henrici. (Krainsky, A., Centrbl. Bakt. II, 41, 1914, 639-688.)

Aerial mycelium: Filaments may show fine, long, narrow spirals. Conidia oval, $1.0-1.2$ by $1.1-1.5 \mu$.

Synthetic agar: Growth thin, gray, spreading. Aerial mycelium white, becoming drab-gray.

Nutrient agar: Growth cream-colored. Aerial mycelium thin.

Glucose agar: Growth yellowish, spreading. No aerial mycelium.

Potato: Growth abundant, wrinkled, cream-colored with greenish tinge. Gelatin: Liquefaction; with small, cream-colored flakes in liquid.

Milk: Ring brownish; coagulation; peptonization in 25 to 30 days, becoming faintly alkaline.

Starch: Growth thin, colorless, spreading. Aerial mycelium gray. Hydrolysis.

Glucose broth: Ring gray, with grayish colonies in bottom of tube.

Nitrate: Reduction to nitrite.

Temperature: Optimum $37^{\circ} \mathrm{C}$.

Antagonistic properties: Limited.

Remarks: Brown to dark brown soluble pigment formed.

34. Streptomyces canescus Hickey et al. (Hickey, R. J., Corum, C. J., Hidy, P. H., Cohen, I. R., Nager, U. F. B., and Kropp, E., Antibiotics \& Chemotherapy, 2, 1952, 472-483.)

Aerial mycelium: At first white, becoming, on sporulation, gray-white to gray. Conidiophores straight or curved, not forming any spirals, richly septate. Spores globose, $1.0-1.3$ by $1.3-2.6 \mu$.

Potato: Growth light gray, spreading, wrinkled. Deep brown pigment diffused throughout.

Gelatin: Liquefaction rapid.

Milk: At $36^{\circ} \mathrm{C}$ becomes alkaline ( $\mathrm{pH}$ 8.4). Soft, rennet curd formed after 48 hours is completely peptonized in 12 days.

Starch: Hydrolysis strong.

Nitrate: No reduction. 
Egg medium: Surface growth tan, very wrinkled. No sporulation observed after 10 days' incubation; small amount of white sporulation observed in 14 days. Soluble brown pigment formed. After 21 days, odor of hydrogen sulfide detected. Liquefaction after 28 days very slow.

Bennett's agar: Colonies circular, effuse to convex, edge filamentous; powdery aerial mycelium; varying from gray-white to gray; reverse brown. No diffusible pigment.

Sabouraud's agar: Growth first white, dull-shiny, spreading, translucent; reverse tan. After 7 days' incubation, growth beaded, slightly wrinkled at base of the slant, grayish white; reverse tan to amber. Amber pigment diffused throughout medium. After 14 days aerial mycelium faintly greenish.

Calcium malate agar: Mycelium gray to rose-gray; reverse yellow to tan; digestion of calcium malate slight at edge of colony. No soluble pigment.

Temperature: Optimum $36^{\circ} \mathrm{C}$.

Antagonistic properties: Produces ascosin.

Source: Contaminated fungus plate.

Remarks: The organism utilizes the following as sole carbon sources: dextrose, arabinose, trehalose, xylose, sucrose, maltose, galactose, dextrin, soluble starch, mannitol, glycerine, and salicin. No growth observed with sorbose, melizatose, dulcitol, rhamnose, sorbitol, melibiose, phenol, raffinose, and lactose.

35. Streptomyces fimicarius (Duché) Waksman and Henrici. (Duché, J., Les actinomyces du groupe albus, P. Lechevalier, Paris, 1934.)

Synthetic agar: Growth yellowish masses, with yellowish white aerial mycelium; reverse orange-colored; faint yellowish soluble pigment.

Nutrient agar: Growth cream-colored with white aerial mycelium; reverse yellowish.

Potato: Growth cream-colored to yellowish with whitish aerial mycelium; reddish-brown pigmentation.

Gelatin: Punctiform colonies with whitish aerial mycelium; soluble pigment reddish. Liquefaction.

Milk: Growth colorless, becoming covered with whitish aerial mycelium; slow peptonization of milk, which becomes rose-colored, finally changing to brownish red.

Asparagine agar: Growth cream-colored with whitish aerial mycelium; reverse cream-colored to slight ocher.

Asparagine solution: Vegetative filaments $0.5-0.6 \mu$ long; branching aerial mycelium $0.8-1.0 \mu$, forming numerous conidia; flaky growth produced on bottom; surface growth becomes covered with a white aerial mycelium; reverse brownish red. 
Synthetic solution: Cream-colored punctiform growth with yellowish aerial mycelium; no soluble pigment.

Nutrient solution: Growth whitish, flakes throughout liquid; yellowish pigment.

Tyrosine medium: Growth white with yellowish reverse; yellowish soluble pigment.

Coagulated serum: Growth cream-colored, with whitish aerial mycelium; liquefaction rapid.

Antagonistic properties: Positive.

Remarks: Abundant growth on neutral and acid media; whitish aerial mycelium; marked odor; soluble brownish red pigment. This species seems to form the transition type between the Streptomyces albus group and the Streptomyces chromogenus group.

36. Streptomyces felleus Lindenbein (Lindenbein, W., Arch. Mikrobiol., 17, 1952, 361-383.)

Aerial mycelium: Hyphae, long, straight, branching. Spores spherical.

Synthetic agar: Growth good, effusive, smooth, with yellow-brown reverse. Aerial mycelium velvety gray-white. Soluble pigment yellowish brown.

Nutrient agar: Growth colorless, diffuse; brownish yellow reverse. No aerial mycelium. Soluble pigment light brownish yellow.

Glucose agar: Growth good, effused, crumb-like, yellowish brown. Aerial mycelium powdery gray-white. Soluble pigment light brown.

Potato: Growth strong, brownish yellow. Aerial mycelium powdery. Faint reddish pigment around growth.

Gelatin: Growth colorless. No aerial mycelium. No soluble pigment. No liquefaction.

Milk: Growth good, brownish to orange. Aerial mycelium velvety graywhite. Peptonization medium.

Starch: Growth lichnoid, colorless. Aerial mycelium powdery white. No soluble pigment. Hydrolysis strong.

Cellulose: No growth.

Glucose broth: Fine sediment with some flakes. Later a colorless ring on surface. No aerial mycelium and no soluble pigment.

Glucose-asparagine agar: Growth thin, colorless, with yellowish reverse. Aerial mycelium powdery gray-white. Soluble pigment brownish.

Calcium malate agar: Growth crumb-like, colorless to yellowish. Aerial mycelium powdery gray-white. Soluble pigment yellowish brown.

Odor: Typical earthy.

Antagonistic properties: Produces picromycin. 
37. Streptomyces achromogenes Okami and Umezawa. (Okami, Y., and Umezawa, H., Japan. Med. J., 6, 1953.)

Aerial mycelium: Fine branching aerial hyphae, no spirals. Spores cylindrical.

Synthetic glycerol agar: Colorless to brownish, restricted growth; scant white to dark grayish-colored aerial mycelium; brown soluble pigment.

Glucose-asparagine agar: Growth yellowish brown, restricted; aerial mycelium scant, yellowish white; reverse of growth brown; soluble pigment none or slightly brown.

Nutrient agar: Growth wrinkled, elevated, colorless to brownish; no aerial mycelium; no soluble pigment.

Potato: Growth yellowish brown to brownish, fine, wrinkled; aerial mycelium white, powdery; no soluble pigment at first, later reddish brown.

Gelatin: Growth yellowish brown, restricted; no aerial mycelium; slightly brown soluble pigment; liquefaction very weak.

Milk: Surface growth poor; no aerial mycelium; no soluble pigment; coagulation and slow peptonization.

Egg media: Growth reddish brown, wrinkled; no aerial mycelium; no soluble pigment.

Loeffler's serum media: Growth elevated, wrinkled, colorless to brownish; no aerial mycelium; no soluble pigment; no liquefaction.

Blood agar: Growth brownish, wrinkled, poor; no aerial mycelium; no soluble pigment; no hemolysis.

Nitrate: Reduction to nitrite.

Antagonistic properties: Produces an antiviral agent, achromoviromycin.

Remarks: This culture resembles Streptomyces diastaticus and Streptomyces fimicarius. This strain differs from the former in the points of spiral formation, hemolysis, liquefaction of gelatin and proteolytic action of milk; it differs from the latter in the points of liquefaction of coagulated serum. This species is characterized by the brown pigmentation on synthetic agar only.

38. Streptomyces noursei Hazen. (Unpublished. Preliminary data by Hazen, E. L., and Brown, R., Proc. Soc. Exp. Biol. Med., 76, 1951, 93.)

Vegetative growth: Good gray to brown growth, much folded on various organic and certain synthetic media; diffusible pink to purple pigment frequently produced.

Aerial mycelium: Well developed white to shell-pink; strajght, curved and spiral-forming sporulating hyphae. Spores round to oval.

Synthetic agar: Scanty, colorless, flat growth, no aerial mycelium.

Glucose agar: Good, folded growth. White aerial mycelium, turning gray. Reverse of growth brown, medium often becoming darkened throughout. 
Occasionally pomegranate-purple soluble pigment is formed.

Potato: Good folded growth, with chalky white aerial mycelium. At $35^{\circ}-36^{\circ} \mathrm{C}$, a reddish purple pigment is formed.

Gelatin: Rapid liquefaction.

Milk: Coagulation, followed by peptonization.

Starch agar: Good growth, in form of discrete colonies with white aerial mycelium in center and periphery colorless and embedded. Hydrolysis.

Cellulose: Poor growth.

Nitrate: Traces of nitrite produced.

Glucose-asparagine agar: Wrinkled tan growth with gray and white knoblike projections. Reverse of growth dark gray. At $35^{\circ}-36^{\circ} \mathrm{C}$, scant, shellpink aerial mycelium; limited shell-pink diffusible pigment.

Blood agar: Good growth, consisting of convex, lobate colonies, with central perforation. Heavy, chalky white aerial mycelium. No hemolysis, but darkening of blood.

Honey broth: Heavy, white ring on surface; flocculent sediment. Broth clear.

Antagonistic properties: Produces an antifungal agent, active against various yeast-like and filamentous fungi, nystatin, and an antibacterial agent, phalamycin.

39. Streptomyces roseochromogenus (Krainsky, emend. Jensen) Waksman and Henrici. (Jensen, H. L., Proc. Linnean Soc. N. S. Wales, 56, 1931, 359.)

Vegetative growth: Pigmented red to rose-colored; produces dark brown soluble substance.

Aerial mycelium: Well developed. Sporophores produce numerous open and closed spirals. Spores $1.0-1.2$ by $1.5-3.0 \mu$.

Synthetic agar: Growth thin, spreading, colorless. Aerial mycelium thin, pale, brownish.

Nutrient agar: Growth white, becoming yellowish.

Potato: Growth much-wrinkled, brownish.

Gelatin: Small cream-colored colonies in bottom of liquid. Soluble brown pigment. Liquefaction medium.

Milk: Coagulation limited; peptonization slow.

Starch: Growth colorless, spreading; hydrolysis good.

Glucose broth: Ring cream-colored, with flaky sediment.

Nitrate: Reduction to nitrite.

Antagonistic properties: Active against various bacteria; produces an antibiotic roseomycin.

40. Streptomyces cinnamonensis Okami, Madeda, Kosaka, Taya, and Umezawa. (Okami, Y., Madeda, K., Kosaka, H., Taya, O., and Umezawa, H., Japan. Med. J., 6, 1953.) 
Vegetative growth: Fine branching mycelium.

Aerial mycelium: Diameter $0.8-1.2 \mu$; long filamentous mycelium; no spirals. Spores elliptical to oval.

Synthetic glycerol agar: Growth colorless, spreading; scant white aerial mycelium or white with pale cinnamon-pinkish (Rdg. XXIX) to light brownish vinaceous tinge (Rdg. XXXIX); no soluble pigment.

Nutrient agar: Growth colorless to dark; no aerial mycelium; none or slightly brown soluble pigment.

Potato: Growth light cream to dark-colored; no aerial mycelium. No soluble pigment; later black pigment produced around growth.

Loeffler's serum media: Growth colorless to dark-colored; no aerial mycelium; soluble brown pigment; no liquefaction.

Gelatin: Growth colorless to dark brownish; aerial mycelium in form of white patches; soluble pigment brown; liquefaction none or very slow.

Milk: Growth cream-colored to brownish surface ring; no aerial mycelium or scant white; no soluble pigment or slightly brown; no coagulation; peptonization none or very slow.

Starch agar: Growth colorless, spreading; aerial mycelium white with pinkish tinge; no soluble pigment; good hydrolysis.

Cellulose: No decomposition.

Nitrate: No reduction.

Glucose-asparagine agar: Growth colorless to light cream-colored; white to white-pinkish cinnamon aerial mycelium to light brownish vinaceous tinge. No soluble pigment.

Blood agar: Growth dark to brownish; no aerial mycelium; soluble brown pigment; no hemolysis.

Antagonistic properties: Produces an antibiotic active against mycobacteria identical with actithiazic acid or thiozolidone.

Remarks: The culture resembles S. roseochromogenus, in the color of the growth, and in points of none or slow liquefaction of coagulated serum and gelatin. It differs in that $S$. roseochromogenus forms numerous open spirals and produces nitrite from nitrate.

The following carbon sources were utilized: sucrose, mannose, dextrin, galactose, glycerol, fructose, glucose, maltose, mannitol, xylose, and sodium succinate. Arabinose, esculin, rhamnose, dulcitol, sodium acetate, inulin, and lactose were not utilized. Salicin and raffinose were negative.

41. Streptomyces olivochromogenus (Bergey et el.) Waksman and Henrici. (Waksman, S. A., Soil Sci., 8, 1919, 106.)

Aerial mycelium: Filaments with numerous close spirals. Conidia oval or elliptical.

Synthetic agar: Growth white, spreading. Aerial mycelium ash-gray with brownish tinge. 
Nutrient agar: Growth wrinkled, brown, becoming gray-green.

Glucose agar: Growth abundant, natal-brown to almost black, entire margin.

Potato: Colonies small, wrinkled, black.

Gelatin: Surface growth cream-colored, spreading. Liquefaction rapid.

Milk: Ring dark brown; coagulation; peptonization, becoming alkaline.

Starch: Growth transparent, spreading. Hydrolysis.

Glucose broth: Growth thin, brown; flaky sediment.

Nitrate: Reduction to nitrite faint.

Temperature: Optimum $37^{\circ} \mathrm{C}$.

Antagonistic properties: Positive.

Remarks: Soluble brown pigment formed.

42. Streptomyces resistomycificus Lindenbein (Lindenbein, W., Arch. Mikrobiol., 17, 1952, 361-383.)

Aerial mycelium: Hyphae long with curling tips. Spores short, oval. Pigment of mycelium becomes pink to pink-gray.

Synthetic agar: Growth good, crumb-like, dark brown. Aerial mycelium velvety chalk-white, later ash-gray. Soluble pigment red-brown.

Nutrient agar: Growth good, crumb-like. Aerial mycelium powdery bluegray. Soluble pigment dark brown.

Glucose agar: Growth crumb-like with dark brown reverse. Aerial mycelim velvety chalk-white. Soluble pigment reddish to dark brown.

Potato: Growth good, brownish black. Aerial myeclium powdery, reddish white. Soluble pigment dark brown.

Gelatin: Growth good, dark brown. Aerial mycelium powdery whitegray. Soluble pigment chestnut-brown. Liquefaction good.

Milk: Growth good, lichnoid; dark brown reverse. Aerial mycelium velvety chalk-white, later yellowish red. Soluble pigment dark brown. Peptonization none or slight.

Starch: Growth good, lichnoid; reddish brown reverse. Aerial mycelium velvety gray-white, later becoming red-gray. Soluble pigment lacking or very equally reddish brown. Hydrolysis strong.

Cellulose: No growth.

Glucose broth: Colonies large, floating, light yellow. Aerial mycelium cottony white to red. Soluble pigment light yellow.

Glucose-asparagine agar: Growth crumb-like; light brown reverse. Aerial mycelium velvety ash-gray. Soluble pigment yellow-brown.

Calcium malate agar: Growth strong, crumb-like; dark brown reverse. Aerial mycelium velvety ash-gray. Soluble pigment ash-gray.

Antagonistic properties: Produces resistomycin, which is present in the mycelium and which is active against gram-positive bacteria. 
43. Streptomyces diastatochromogenes (Krainsky) Waksman and Henrici. (Krainsky, A., Centrbl. Bakt. II, 41, 1914, 662.)

Aerial mycelium: Conidia spherical or oval, about $1.2 \mu$.

Nutrient agar: Colonies medium-sized with white to gray aerial mycelium.

Glucose agar: Colonies medium-sized, colorless, with gray aerial mycelium.

Potato: Colonies light gray; aerial mycelium gray; medium colored black. Gelatin: Colonies light gray. Soluble brown pigment formed. Liquefaction. Starch: Same as on glucose agar. Weakly diastatic.

Cellulose: No growth.

Glucose broth: Flaky colonies in depth at first, later also over surface.

Calcium malate agar: Colonies medium-sized, colorless, with gray aerial mycelium.

Temperature: Optimum $35^{\circ} \mathrm{C}$.

Antagonistic properties: Very strong.

Remarks: Tyrosinase formed.

44. Streptomyces flavochromogenes (Krainsky) Waksman and Henrici. (Krainsky, A., Centrbl. Bakt. II, 41, 1914, 662.)

Aerial mycelium: Conidia oval, $1.7 \mu$.

Nutrient agar: Aerial mycelium formed late, at first white, later gray. Gray soluble pigment formed.

Glucose agar: Brown soluble pigment formed.

Potato: Colonies yellow; white aerial mycelium.

Gelatin: Colonies yellowish. Liquefaction slight.

Starch: Colonies yellow; aerial mycelium white. Weakly diastatic.

Cellulose: Growth slow.

Glucose broth: Fine flakes, with small spherical colonies adherent to glass. Medium colored brown.

Nitrate: Reduction to nitrite.

Esculin: Acted upon.

Calcium malate agar: Colonies yellow with white aerial mycelium forming late.

Temperature: Optimum $35^{\circ} \mathrm{C}$.

Remarks: Tyrosinase formed.

45. Streptomyces bikiniensis Johnstone and Waksman. (Johnstone, D. B., and Waksman, S. A., J. Bacteriol., 55, 1948, 317-326.)

Aerial mycelium: Hyphae straight, branched heterogeneously. No spirals. Conidia oval.

Synthetic agar: Growth white, becoming pallid neutral gray, with white 
tinge. Aerial mycelium abundant. Soluble pigment light brown. Superficial droplets amber-colored.

Nutrient agar: Growth luxuriant; aerial mycelium moderate, white. Soluble pigment deep brown.

Glucose agar: Aerial mycelium gray. Soluble pigment deep brown.

Potato: Growth wrinkled and raised, pale ocherous buff; soluble pigment brown to black.

Gelatin: Liquefaction slight.

Milk: Surface growth patchy, white; aerial mycelium gray. Hydrolysis gradual.

Starch: Growth abundant; aerial mycelium white, becoming gray. Hydrolysis slight.

Nutrient broth: Surface pellicle, abundant, white; soluble pigment deep brown.

Glucose-asparagine agar: Growth luxuriant; aerial mycelium white to mouse-gray. Soluble pigment light amber.

Antagonistic properties: Strongly antagonistic. Produces streptomycin.

Source: Soil from Bikini atoll.

45a. Streptomyces mirabilis Ruschmann (Ruschmann, G. Die Pharmazie, $7,1952,542-550,639-648,823-831$.)

Aerial mycelium: White, fluffy layer, consisting of abundant aerial mycelium and spores. Hyphae straight, without spirals or curvature; sporulation takes place after 6-7 days.

Nutrient agar: Poor growth; slimy surface, resembling that of bacteria. No sporulation.

Glucose agar: Grayish brown, shiny growth. No aerial mycelium even after 14 days. Soluble brown pigment.

Potato: Good, lichnoid growth; dark brown to black, soluble pigment.

Gelatin: Good flaky growth. Rapid liquefaction. Dark brown to black soluble pigment.

Milk: Surface growth with white, fluffy aerial mycelium. Coagulation and peptonization. Liquefied portion colored black. Reaction of medium unchanged.

Czapek's solution: Limited growth in the form of a few surface colonies, sinking rapidly to bottom.

Carrot juice agar: Good growth in form of round colonies, covered with white aerial mycelium. Each colony produces 3 to 5 cracks or holes in center.

Temperature: Optimum at $29^{\circ} \mathrm{C}$. No growth at $37^{\circ} \mathrm{C}$.

Oxygen requirement: Quite considerable.

Fats: Readily utilized.

Antagonistic properties: Produces miramycin. Antagonistic effect strong- 
est in freshly isolated cultures. Property lost on cultivation; activity upon gram-negative bacteria is lost at first, then upon gram-negative rods; cocci remain most sensitive.

Remarks: Highly proteolytic and lipolytic. Grows best on complex organic media, at slightly acid reaction of $\mathrm{pH}$ 6.0-6.6.

46. Streptomyces antibioticus (Waksman and Woodruff) Waksman and Henrici. (Waksman, S. A., and Woodruff, H. B., J. Bacteriol., 42, 1941, 232, 246.)

Aerial mycelium: Spore-bearing hyphae produced in the form of straight aerial hyphae. Conidiophores arranged in clusters; no spirals formed. Conidia nearly spherical to somewhat elliptical.

Synthetic agar: Growth thin, whitish. Aerial mycelium thin, gray.

Nutrient agar: Production of dark pigment at early stage of growth is very characteristic. Growth brownish, thin, with yellowish gray to yellowish green aerial mycelium.

Potato: Growth folded, brown, with thin black ring on plug, fading into a bluish tinge. No aerial mycelium.

Carrot: Growth cream-colored to faint brownish. No aerial mycelium. No pigment.

Gelatin: Surface growth dark brown; patches of gray aerial mycelium. Dark pigment produced, which gradually diffuses into the unliquefied part of the gelatin. Liquefaction at first very slow, later becoming rapid.

Milk: Surface ring thick, brownish. Aerial mycelium mouse-gray with greenish tinge; growth becomes brown, especially in dried portions adhering to glass. No reaction change, no coagulation of milk, no clearing; whitish sediment at bottom of tube. Old cultures: Heavy growth ring on surface of milk, heavy precipitation on bottom; liquid brownish to black in upper portion.

Odor: Very characteristic of soil.

Antagonistic properties: Has a marked antagonistic effect on gram-positive and gram-negative bacteria, much more on the former than on the latter, as well as on actinomycetes. It is also active against fungi, which vary in degree of sensitivity. Produces actinomycin, a specific bacteriostatic and bactericidal substance.

Source: Isolated from soil on Escherichia coli-washed agar plate, using living cells of $E$. coli as the only source of available nutrients.

47. Streptomyces griseocarneus Grundy, Whitman, Hanes, and Sylvester. (Grundy, W. E., et al., Antibiotics \& Chemotherapy, 1, 1951, 309317.)

Vegetative growth: Good with monopodial branching.

Aerial mycelium: Two types. On some media, powdery, suggestive of 
sporulation, but no spores are produced; this type of mycelium usually becomes gray with continued incubation. On other media, the aerial mycelium forms a rather fluffy mat, white in color; it turns slightly pink when sporulation occurs. No spirals have been observed. None of the common media will induce sporulation. It occurs best after 10 to 14 days' incubation on a carbon-free salt agar, to which 0.5 per cent soluble starch has been added. Inositol and mannose will also support sporulation, but they are not as satisfactory carbon sources for this purpose as starch. The only other medium on which spores are found is nitrogen-free synthetic agar to which 0.2 per cent glycine or asparagine is added. The spores are coccoid to oval $1.1-1.6$ by $0.7-1.1 \mu$.

Synthetic agar: Growth sparse, white; aerial mycelium white; no sporulation; no soluble pigment.

Nutrient agar: Growth moderate, cream-colored; no aerial mycelium; soluble pigment light yellow-brown.

Potato: Growth luxuriant, spreading, cream-colored; aerial mycelium gray; soluble pigment light brown turning dark brown after 30 days' incubation.

Gelatin: Growth cream-colored to brown. Liquefaction rapid. Soluble pigment dark brown.

Milk: Growth dark brown to black. No coagulation. Peptonization rapid. Soluble pigment brown.

Starch: Hydrolysis.

Nitrate: Reduction negative.

Glucose-asparagine agar: Growth moderate; aerial mycelium powdery white; no pigmentation, no sporulation.

Calcium malate: Growth moderate, white; aerial mycelium white; no soluble pigment; no sporulation.

Oatmeal agar: Growth luxuriant, brown; aerial mycelium abundant, fluffy, white; no sporulation; no soluble pigment.

Antagonistic properties: Produces hydroxystreptomycin.

Remarks: Carbon sources rapidly utilized: glucose, dextrin, starch, glycerol, calcium malate, and sodium succinate. Several assimilated slowly: mannose, maltose, inositol, and sodium acetate. Others not utilized: xylose, galactose, sorbose, sucrose, cellobiose, melibiose, lactose, mannitol, sorbitol, sodium citrate, and potassium sodium tartrate. Among the several nitrogen sources investigated, $\left(\mathrm{NH}_{4}\right)_{2} \mathrm{HPO}_{4}$, urea, asparagine, glycine, and arginine support moderate to rapid growth; $\mathrm{NaNO}_{3}$ supports slow growth; tryptophane, tyrosine, and methionine are not satisfactory nitrogen sources.

48. Streptomyces viridoflavus $n$. $s p$. Waksman and Taber.

Vegetative growth: Abundant lichnoid growth on most media; color of growth yellow-green, turning olive-green to almost brown. Soluble pigment 
variable. Hyphae on surface of agar $0.7 \mu$ in diameter; in shake flasks 0.7$0.8 \mu$; some of the submerged hyphae are much thicker, reaching $1.6 \mu$ in diameter. Formation of single spores at the end of the submerged sporulating lateral branches. "Spores" form early, germinate readily, even while apparently attached to hyphae.

Aerial mycelium: Aerial hyphae formed in fascicles; greenish yellow in color, turning gray. Tendency to lose property of producing aerial mycelium. Tufts, with some curling of tips produced on certain media. On glucose-asparagine agar, spores produced in chains, in whorls. No sporulation on nutrient or glucose-nutrient agar.

Synthetic agar: Limited growth, cream-colored to yellowish green tinge. Generally, no aerial mycelium. No diffusible pigment. Hyphae penetrate deep into agar.

Nutrient agar: Thin growth, consisting of moist, isolated colonies, gray to light green in color, with green to almost bluish tinge at bottom of slant where colonies are confluent. White to gray nonsporulating aerial mycelium appears much later. No diffusible pigment.

Glucose-nutrient agar: Heavy lichnoid growth, yellowish brown to olivebrown in color. Yellowish to gray aerial hyphae are abundant and appear later, covering the whole surface of growth with a mat. Diffusible pigment brownish or absent.

Glucose-asparagine agar: Moist, flat, yellow to yellow-green colonies growing deep into medium. Aerial hyphae frequently abundant; grayish yellow to sulfur-yellow, later overgrown by white sporulating hyphae. Diffusible pigment absent or faint yellow.

Yeast-glucose agar: Heavy lichnoid growth, dark brown to olive-green in color. Aerial hyphae pale to grayish yellow to greenish yellow, becoming gray with age. Soluble brown pigment.

Potato: Lichnoid growth, brownish to greenish yellow to dark olive-green in color. Aerial hyphae absent, or formed as thin, yellowish layer on drier portions of growth. Soluble pigment absent or dark brown.

Gelatin: Limited growth in form of surface ring, canary-yellow in color. No pellicle formed. Gelatin only slightly liquefied. Brown to dark brown soluble pigment; this property may be lost on cultivation.

Nutrient broth: Growth colorless in clumps on bottom of container; soluble brown pigment, a property which may be lost on cultivation.

Milk: Light yellow to brown surface ring. No coagulation; gradual peptonization.

Starch: Good hydrolysis.

Carbon source: With yeast extract-mineral agar, there was no growth over control on sucrose, lactose, or rhamnose; good growth on mannose and glucose.

Cellulose: Limited growth, no destruction of cellulose. 
Antagonistic properties: Produces antibiotic substances, one of which is candicidin-like material.

Source: Soil.

Remarks: Several strains of this organism have been isolated. They differ in the pigmentation of gelatin and in the relative abundance of aerial mycelium.

49. Streptomyces globosus (Krassilnikov) comb. nov. (Krassilnikov, N. A., Actinomycetales, Akad. Nauk. USSR, Moskau, 1941, 58.)

Vegetative growth: Colonies dark brown producing soluble brown pigment into medium.

Aerial mycelium: Dark gray, fine velvety. Sporophores straight, short; spores spherical.

Gelatin: Liquefaction weak.

Milk: Peptonization.

Starch: Hydrolysis.

Sucrose: No inversion.

Antagonistic properties: No activity against mycobacteria.

Source: Soil, food products, potatoes.

50. Streptomyces cylindrosporus (Krassilnikov) comb. nov. (Krassilnikov, N. A., Actinomycetales, Akad. Nauk. USSR, Moskau, 1941, 57.)

Vegetative growth: Cultures readily lose capacity to produce spores.

Aerial mycelium: Well-developed, cottony or velvety, grayish white. Sporophores straight, spores cylindrical, $1.0-1.7$ by $0.7 \mu$.

Synthetic agar: Colonies velvety, dark brown or chocolate, with whitegray aerial mycelium.

Nutrient agar: Growth dark brown, with white aerial mycelium.

Gelatin: Liquefaction medium.

Milk: Coagulation limited, peptonization weak, milk becoming brown to almost black.

Starch: Hydrolysis weak.

Cellulose: No growth.

Nitrate: Reduction strong to weak.

Sucrose: No inversion.

Antagonistic properties: None.

51. Streptomyces viridochromogenes (Krainsky) Waksman and Henrici. (Krainsky, A., Centrbl. Bakt. II, 41, 1914, 639-688.)

Aerial mycelium: Hyphae with numerous open spirals, $3-5 \mu$ in diameter, occurring as side branches; spores short, oval or spherical, 1.25-1.5 $\mu$. 
Synthetic agar: Growth spreading, cream-colored with dark center, becoming dark green; reverse yellowish to light cadmium. Aerial mycelium abundant, spreading, white, becoming light green.

Nutrient agar: Growth abundant, restricted, gray, with greenish tinge.

Glucose agar: Growth abundant, spreading, wrinkled, gray, becoming black.

Potato: Growth abundant, gray-brown.

Gelatin: Surface growth cream-colored, becoming greenish. Liquefaction slow.

Milk: Surface growth dark brown; coagulation, peptonization.

Starch: Colonies circular, spreading, yellowish. Hydrolysis positive.

Glucose broth: Dense, solid ring, brownish, becoming dark green.

Nitrate: Reduction to nitrite.

Temperature: Optimum $37^{\circ} \mathrm{C}$.

Antagonistic properties: Active upon fungi.

Remarks: Soluble brown pigment formed.

52. Streptomyces purpeochromogenus (Waksman and Curtis) Waksman and Henrici. (Waksman, S. A., Soil Sci., 1, 1916, 113.)

Aerial mycelium: Branching mycelium and hyphae with few imperfect spirals. Conidia spherical, $0.75-1.0 \mu$ in diameter.

Synthetic agar: Growth slow, restricted, smooth, gray, becoming brown with purplish tinge; center raised. Margin yellow.

Nutrient agar: Growth gray to brownish, becoming dark brown, almost black.

Glucose agar: Growth abundant, restricted, gray, becoming brown to dark brown.

Potato: Growth restricted, orange to orange-red.

Gelatin: Surface growth slow, brownish. Liquefaction slow.

Milk: Ring dark brown; coagulation; peptonization slow, with faintly alkaline reaction.

Starch: Colonies small, dark brown. Hydrolysis slight.

Glucose broth: Sediment slight, flaky.

Nitrate: No reduction to nitrite.

Temperature: Optimum $25^{\circ} \mathrm{C}$.

Antagonistic properties: Active against various bacteria.

Remarks: Soluble dark brown pigment formed.

53. Streptomyces phaeochromogenus (Conn) Waksman and Henrici. (Conn, H. J., N. Y. Agr. Exp. Sta. Tech. Bull. 60, 1917, 60.)

Aerial mycelium: Branching filaments and hyphae, spirals narrow, open, elongated, sinistrorse. 
Synthetic agar: Growth colorless, becoming brown to almost black. Aerial mycelium abundant, white with brownish shade.

Nutrient agar: Growth thin, cream-colored, becoming gray.

Glucose agar: Growth restricted, much-folded, brown.

Potato: Growth brown to almost black.

Gelatin: Surface growth abundant, spreading, cream-colored, becoming brown. Liquefaction slow.

Milk: Ring dark, almost black; coagulation, with slow peptonization, faintly alkaline reaction.

Starch: Growth spreading, brownish, becoming brown. Hydrolysis.

Glucose broth: Pellicle dense, wrinkled.

Nitrate: Reduction to nitrite.

Temperature: Optimum $25^{\circ} \mathrm{C}$.

Antagonistic properties: Strong.

Remarks: Soluble brown pigment formed.

54. Streptomyces aureus (Waksman and Curtis) Waksman and Henrici. (Waksman, S. A., and Curtis, R. E., Soil Sci., 1, 1916, 124.)

Aerial mycelium: Shows numerous spirals. Conidia spherical to oval, $0.6-1.0$ by $0.8-1.4 \mu$.

Synthetic agar: Growth thin, spreading, colorless. Aerial mycelium thin, powdery, gray, becoming cinnamon-drab.

Nutrient agar: Growth restricted, gray.

Glucose agar: Growth spreading, light orange, raised center, hyaline margin.

Potato: Growth abundant, wrinkled, brown, becoming black.

Gelatin: Fair, cream-colored surface growth, becoming brown, spreading. Liquefied.

Milk: Ring black. No coagulation. Peptonization doubtful.

Starch: Growth thin, transparent, spreading. Hydrolysis positive.

Glucose broth: Ring thin, brownish; flaky sediment.

Nitrate: Reduction to nitrite.

Temperature: Optimum $25^{\circ} \mathrm{C}$.

Antagonistic properties: Produces fungicidin RAW (antimycoin), a substance active against various fungi. Some strains produce luteomycin.

Remarks: Soluble brown pigment formed in organic media.

55. Streptomyces tanashiensis Hata, Ohki, and Higuchi (Hata, T., Ohki, N., and Higuchi, T., J. Antibiotics [Japan] 5, 1952, 529-534, 313-325.)

Aerial mycelium: Hyphae almost straight; lightly open spirals; spores spherical to oval, 1.0 by $1.2 \mu$.

Synthetic agar: Growth grayish yellow. Aerial mycelium white-gray turning brownish gray. Soluble pigment light yellow. 
Potato: Growth brown; aerial mycelium dark gray to whitish gray; soluble pigment dark brown.

Gelatin: Brown pigment. Rapid liquefaction.

Milk - Yellowish surface ring; coagulation and peptonization.

Starch Hydrolysis.

Nitrate: No reduction.

Tyrosinase reaction: Positive.

Optimum $\mathrm{pH}: 5.8-6.5$.

Antagonistic properties: Produces luteomycin.

Remarks: Resembles Stretomyces aureus and Streptomyces antibioticus. Carbon sources: starch most suitable, followed by glycerol. Nitrogen source: peptone and meat extract best.

56. Streptomyces erythrochromogenes (Krainsky) Waksman and Henrici. (Krainsky, A., Centrbl. Bakt. II, 41, 1914, 639-688.)

Aerial mycelium: Conidia oval, about $2.0 \mu$ long.

Nutrient agar: Soluble pigment brown. Aerial mycelium white.

Glucose agar: Red pigment formed.

Potato: Aerial mycelium gray. Medium colored black.

Gelatin: Colony growth slow. Liquefaction. Soluble brown pigment formed.

Starch: Soluble pigment on old cultures rose. Diastatic action weak.

Cellulose: No growth.

Glucose broth: Growth abundant. Colonies floating, later a pellicle is formed. Soluble pigment brown.

Nitrate: Reduction slight.

Calcium malate agar: Colonies circular, with aerial mycelium grayishwhite-margined.

Temperature: Optimum $30^{\circ} \mathrm{C}$.

Antagonistic properties: Active against various bacteria.

57. Streptomyces collinus Lindenbein (Lindenbein, W., Arch. Mikrobiol., 17, 1952, 361-383.)

Aerial mycelium: Produces spirals, oval spores.

Synthetic agar: Growth good, crumb-like, light brown to red-brown in reverse. Aerial mycelium chalk-white. Soluble pigment yellow-brown, later becoming reddish brown.

Nutrient agar: Growth good, crumb-like, dark brown. Aerial mycelium powdery gray-white. Soluble pigment dark brown.

Glucose agar: Growth good, reverse yellow-brown and red. Aerial mycelium velvety and chalk-white. Soluble pigment chestnut-brown. 
Potato: Growth good. Aerial mycelium chalk-white. No soluble pigment.

Gelatin: Growth good, dark brown. No aerial mycelium. Soluble pigment dark brown. Liquefaction rapid.

Milk: Growth good; dark brown reverse. Aerial mycelium chalk-white, later ash-gray. Soluble pigment dark brown. No peptonization.

Starch: Colonies large, reddish, with reddish yellow reverse. Aerial mycelium velvety chalk-white. Hydrolysis medium.

Cellulose: Growth good.

Glucose broth: Sedimentary growth light yellow. No aerial mycelium. Soluble pigment brownish yellow.

Glucose-asparagine agar: Growth good, crumb-like, with reverse irregularly brown-purple to brown-yellow. Aerial mycelium chalk-white, later ash-gray. Soluble pigment carmine-red, later brown-red.

Calcium malate agar: Growth good, crumb-like, yellow-brown to red in reverse. Aerial mycelium velvety chalk-white. Soluble pigment yellowbrown.

Antagonistic properties: Produces an active pigment.

58. Streptomyces lavendulae (Waksman and Curtis) Waksman and Henrici. (Waksman, S. A., and Curtis, R. E., Soil Sci., 1, 1916, 126.)

Aerial mycelium: Hyphae coarse, branching. Spirals close, $5-8 \mu$ in diameter. Conidia oval, $1.0-1.2$ by $1.6-2.0 \mu$.

Synthetic agar: Growth thin, spreading, colorless. Aerial mycelium cottony, white, becoming vinous-lavender.

Nutrient agar: Growth wrinkled, gray.

Potato: Growth thin, wrinkled, cream-colored to yellowish. Color of potato black.

Gelatin: Surface growth creamy to brownish. Liquefaction.

Milk: Ring cream-colored. No coagulation; peptonization, with strong alkaline reaction.

Starch: Growth restricted, glistening, transparent. Hydrolysis positive.

Glucose broth: Sediment abundant, flaky.

Nitrate: Reduction to nitrite.

Temperature: Optimum $37^{\circ} \mathrm{C}$.

Antagonistic properties: Certain strains of this organism produce antibiotics. One such antibiotic, streptothricin, is active both in vitro and in vivo against various gram-positive and gram-negative bacteria, fungi, and actinomycetes. Certain other strains produce an antiviral agent, ehrlichin.

Remarks: Soluble brown pigment formed in organic media.

59. Streptomyces venezuelae Ehrlich, Gottlieb, Burkholder, Anderson, and Pridham. (Ehrlich, J., Gottlieb, D., Burkholder, P. R., Anderson, L. E., 
and Pridham, T. G., J. Bacteriol., 56, 1948, 467-477; Pridham, T. G., and Gottlieb, D., J. Bacteriol., 56, 1948, 107-114.)

Vegetative growth: Hyphae colorless, monopodial branches, $0.9-1.8 \mu$ in diameter.

Aerial mycelium: Straight or slightly and irregularly curved, 1.0-1.8 $\mu$ in diameter, lavender under microscope, gray to light tan or pink without magnification. Spores oval to oblong, $0.4-0.8$ by $0.7-1.6 \mu$.

Synthetic agar: Growth light lavender-colored.

Glucose agar: Soluble pigment dark.

Potato: Growth abundant, gray.

Gelatin: Liquefaction rapid; soluble pigment dark.

Milk: Peptonization; soluble pigment dark.

Starch: Growth white to lavender.

Nitrate: Reduction positive.

Dorset's egg medium: Growth white to gray.

Loeffler's serum: Growth dark brown.

Tyrosine agar: Reaction positive.

Antagonistic properties: Produces chloramphenicol, a neutral compound active against various gram-positive and gram-negative bacteria, rickettsiae, and larger viruses.

Remarks: Good growth with xylose, arabinose, rhamnose, d-glucose, dmannose, d-fructose, d-galactose, cellobiose, starch, dextrin, glycerol, acetate, citrate, succinate, and salicin. Slight or no growth with d-ribose, sucrose, raffinose, inulin, erythritol, dulcitol, mannitol, sorbitol, inositol, and malate. No growth with formate, oxalate, tartrate, salicylate, phenol, $o$-cresol, $m$-cresol, $p$-cresol.

Syn. S. pheochromogenus var. chloromyceticus Okami.

60. Streptomyces virginiae Grundy, Whitman, Rdzok, Rdzok, Hanes, and Sylvester. (Grundy, W. E., Whitman, A. L., Rdzok, E. C., Rdzok, E. J., Hanes, M. E., and Sylvester, J. C., Antibiotics \& Chemotherapy, 2, 1952, 399-408.)

Vegetative growth: Cream-colored to light brown on complex media; soluble pigment diffusible, light brown. On synthetic media growth white to cream-colored, with pink or grayish lavender aerial mycelium. Undulating mycelium about $1 \mu$ in diameter with short thinner side branches.

Aerial mycelium: Aerial hyphae long, grayish pink or lavender. Most of the sporulating hyphae are straight, but occasionally a spiral is observed at or near the tip of the hypha. These spirals vary from tightly closed knots to loose open spirals. Spores cylindrical, $1.1-1.5$ by $0.75-1.0 \mu$.

Synthetic glucose agar: Growth sparse, cream-colored; aerial mycelium light grayish lavender. No soluble pigment.

Nutrient agar: Growth sparse, white, turning cream-colored; aerial myce- 
lium white, turning light grayish lavender when sporulation occurs. No soluble pigment.

Potato: Growth abundant, spreading, brownish; aerial mycelium grayish lavender; browning of the potato.

Gelatin: Surface pellicle gray to brownish; aerial mycelium thin, white. Brown soluble pigment extending as far as liquefied zone. Liquefaction slow.

Milk: Growth brown. No coagulation; peptonization slow. Milk becomes dark gray-brown or black.

Starch: Growth thin colorless; aerial mycelium rose to lavender-colored. Hydrolysis.

Nutrient broth: Ring on surface thin, cream-colored; a few flakes on bottom. Soluble pigment brownish.

Nitrate: Reduction none or limited.

Glucose-asparagine agar: Growth sparse, cream-colored; no aerial mycelium; no sporulation; no pigmentation.

Calcium malate agar: Growth abundant, cream-colored; aerial mycelium white, becoming tinged with grayish lavender; no soluble pigment.

Oatmeal agar: Growth abundant, cream-colored, turning golden brown; aerial mycelium abundant, light rose, turning lavender and gray. Soluble pigment pale yellow, turning light brown.

Temperature: Optimum $28^{\circ}-30^{\circ} \mathrm{C}$.

Antagonistic properties: Produces actithiazic acid.

Remarks: Utilizes glucose, mannose, galactose, maltose, starch, glycerol, sodium acetate, sodium citrate; does not utilize xylose, lactose, sucrose, mannitol, sorbitol, potassium sodium tartrate.

61. Streptomyces reticuli (Waksman and Curtis) Waksman and Henrici. (Waksman, S. A., and Curtis, R. E., Soil Sci., 1, 1916, 118.)

Aerial mycelium: Whorls; spirals formed on glucose agar are sinistrorse. Conidia spherical, $1.0-1.4 \mu$ in diameter.

Synthetic agar: Growth colorless, with yellowish tinge, becoming brownish, spreading. Aerial mycelium thin, white, cottony.

Nutrient agar: Growth wrinkled, gray, becoming brownish.

Glucose agar: Growth restricted, brownish, center raised.

Potato: Growth gray, with black center.

Gelatin: Liquefaction with small, brown flakes.

Milk: Reaction unchanged; coagulation; peptonization.

Starch: Growth brownish gray. Hydrolysis positive.

Glucose broth: Sediment consisting of large colonies.

Nitrate: Reduction to nitrite.

Temperature: Optimum $25^{\circ} \mathrm{C}$. 
Antagonistic properties: Some strains produce neomycin or a neomycinlike substance.

Remarks: Dark brown pigment formed in organic media.

62. Streptomyces netropsis Finlay and Sobin. (Finlay, A. C., and Sobin, B. A., U. S. Pat. 2,586,762, 1952.)

Vegetative growth: Growth slightly elevated, with rough surface and smooth edge. Reverse brown.

Aerial mycelium: White. Sporophores in form of whorls or terminal clusters of short hyphae on tips of short hyphae. Conidia short, cylindrical, 0.7 by $1.3 \mu$.

Synthetic agar: Growth thin, pale olive-buff. Pale vinaceous fawn aerial mycelium. No soluble pigment.

Nutrient agar: Growth moderate to good, light brown; white aerial mycelium, light brown soluble pigment.

Glucose agar: Growth good, dark brown. White aerial mycelium. Brown soluble pigment.

Potato: Growth poor, waxy, wrinkled, brown. No aerial mycelium. Dark brown pigment.

Gelatin: Moderate surface growth. White aerial mycelium. Dark brown soluble pigment. No liquefaction.

Milk: Growth poor. No peptonization.

Starch: Growth moderate, thin; pale olive-buff reverse. White aerial mycelium. No soluble pigment. Strong hydrolysis.

Nitrate: No reduction.

Glucose-asparagine agar: Growth moderate, wrinkled. White aerial mycelium. Soluble brown pigment.

Calcium malate agar: Growth moderate, cream to buff. White aerial mycelium. No soluble pigment.

Antagonistic properties: Produces a basic antibiotic, netropsin.

63. Streptomyces verticillatus (Kriss) comb. nov. (Kriss, A., Microbiologia, $7,1938,111$.)

Vegetative growth: Colorless or slightly brownish, smooth or rough colonies.

Aerial mycelium: Characteristic primary whorl formation of straight sporophores. Spores cylindrical, oblong, $1.0-1.9$ by $0.8 \mu$.

Synthetic agar: Aerial mycelium well-developed, velvety, at first white, later dark gray or gray-green.

Nutrient agar: Growth brown. Soluble pigment brown. No aerial mycelium.

Potato: Soluble pigment brown. 
Gelation: Liquefaction rapid.

Milk: Coagulation and peptonization.

Starch: Hydrolysis.

Cellulose: No growth.

Nitrate: Reduction rapid.

Sucrose: Inversion.

Antagonistic properties: Weak.

Remarks: Streptomyces verticillatus viridans described as substrain.

64. Streptomyces circulatus (Krassilnikov) comb. nov. (Krassilnikov, N. A., Actinomycetales, Akad. Nauk. USSR, Moskau, 1941, 60.)

Vetetative growth: Better on synthetic than on organic media.

Aerial mycelium: Spiral-producing sporophores formed in whorls. Spores cylindrical, oblong, 1.5 by $0.7 \mu$, some rounding up with age of culture.

Synthetic agar: Growth good, producing abundant white aerial mycelium.

Nutrient agar: Growth weak; no aerial mycelium.

Gelatin: Liquefaction weak.

Milk: No coagulation; peptonization slow.

Starch: Hydrolysis weak.

Cellulose: No growth.

Nitrate: Reduction weak.

Sucrose: No inversion.

Antagonistic properties: Limited.

65. Streptomyces rubrireticuli (Waksman) Waksman and Henrici. (Waksman, S. A., Soil Sci., 8, 1919, 146.)

Aerial mycelium: Branching filaments with both primary and secondary whorl formation. Spirals formed on glucose agar. Conidia oval-shaped.

Synthetic agar: Growth abundant, spreading, usually pink. Aerial mycelium thin, rose to pink.

Nutrient agar: Growth red, with yellowish margin, becoming red.

Glucose agar: Entire growth abundant, spreading, rose-red.

Potato: Growth cream-colored, later pink to dark red.

Gelatin: Surface growth yellowish red to dragon-pink. Liquefaction.

Milk: Pellicle abundant, red; coagulation; peptonization. Reaction unchanged.

Starch: Growth white with red tinge. Hydrolysis positive.

Cellulose: Good growth.

Glucose broth: Sediment thin, flaky.

Nitrate: Reduction to nitrite. 
Antagonistic properties: Certain strains of this organism produce an antibiotic, designated as streptin.

Remarks: Soluble dark brown pigment formed in organic media.

66. Streptomyces flavus (Krainsky) Waksman and Henrici. (Krainsky, A., Centrbl. Bakt. II, 41, 1914, 639-688.)

Aerial mycelium: Coarse filaments with branching hyphae. Conidia formed by budding and breaking up of hyphae into oval forms.

Synthetic agar: Colonies circular, yellow or sulfur-yellow. Aerial mycelium straw-yellow.

Nutrient agar: Growth gray, spreading, folded.

Glucose agar: Growth restricted, raised, folded, sulfur-yellow, center shading to brown.

Potato: Growth elevated, much wrinkled, greenish olive.

Gelatin: Small, yellowish masses on surface of liquefied medium.

Milk: Coagulation and peptonization.

Starch: Growth spreading, cream-colored with pink tinge. Hydrolysis marked.

Glucose broth: Colonies in bottom of tube small, white.

Nitrate: Reduction to nitrite slight.

Temperature: Optimum $25^{\circ} \mathrm{C}$.

Antagonistic properties: Weakly antagonistic. Some strains produce actinomycin.

Remarks: Represents a large group of closely related forms.

67. Streptomyces ruber (Krainsky) Waksman and Henrici. (Krainsky, A., Centrbl. Bakt. II, 41, 1914, 639-688.)

Aerial mycelium: Straight, branching, radiating. A few spirals may be formed.

Synthetic agar: Growth abundant, spreading, red. Aerial mycelium abundant, cottony, chrome-orange.

Nutrient agar: Growth restricted, elevated, wrinkled, olive-green.

Glucose agar: Growth restricted, abundant, entire, coral-red.

Potato: Growth elevated, wrinkled, greenish.

Gelatin: Liquefaction, with yellow flakes.

Milk: Dark ring with red tinge; coagulation; peptonization, with alkaline reaction.

Starch: Growth abundant, spreading, red. Hydrolysis.

Glucose broth: Red ring, with spongy colonies on the surface. 
Nitrate: Reduction to nitrite.

Temperature: Optimum $37^{\circ} \mathrm{C}$.

Antagonistic properties: Strongly effective upon various bacteria.

Remarks: Soluble brown pigment formed.

68. Streptomyces niger (Rossi Doria emend. Krassilnikov) comb. nov. (Krassilnikov, N. A., Actinomycetales, Akad. Nauk. USSR, Moskau, 1941, 53.)

Vegetative growth: Not compact, of soft consistency, lumpy, darkcolored. In old cultures, the mycelium is readily disintegrated into fine particles that can serve for reproduction.

Aerial mycelium: Produced only on potato and synthetic agar. Sporophores produced only seldom; form open spiral, with 3-5 turns. Spores oval.

Synthetic agar: Growth black; aerial mycelium dark gray. No soluble pigment.

Nutrient agar: Growth black. Soluble pigment brown.

Gelatin: Not liquefied in 30 days.

Milk: No change.

Starch: No growth.

Cellulose: No growth.

Nitrate: No reduction.

Sucrose: No inversion.

Temperature: Optimum $25^{\circ}-30^{\circ} \mathrm{C}$.

Antagonistic properties: Negative.

Remarks: This is a very unstable species and rapidly dies out. It easily mutates, giving rise to colorless cultures, producing no aerial mycelium.

69. Streptomyces alboniger Hesseltine et al. (Hesseltine, C. W., Porter, J. N., Deduck, N., Hauk, M., Bohonos, N., and Williams, J. H., Mycologia, 1953.)

Vegetative growth: Growth moist, colorless to yellowish, to dark-brown or black.

Aerial mycelium: White to pale olive-buff. Irregularly branched sporophores, erect to flexuous. Spores catenulate, oval, 0.8 by $1.25 \mu$.

Synthetic agar: Growth poor, white; aerial mycelium white. No soluble pigment.

Nutrient agar: Growth poor, moist, smooth, colorless. No aerial mycelium.

Glucose agar: Growth blackish gray; aerial mycelium white. Blackish gray soluble pigment.

Potato: Growth moist, yellow. White aerial mycelium. Dark, greenish black soluble pigment. 
Gelatin: Growth fair. White aerial mycelium. Light yellow soluble pigment. Medium liquefaction.

Milk: Surface ring white, with yellow-green to light yellow-brown below surface. White aerial mycelium. Slow peptonization.

Starch: Growth good. White to pale olive-buff aerial mycelium. Black soluble pigment. Good hydrolysis.

Cellulose: No growth.

Antagonistic properties: Produces puromycin, an antibiotic active upon certain protozoa and certain gram-positive bacteria.

70. Streptomyces abikoensum Umezawa, Tazaki, and Fukuyama. (Umezawa, H., Tazaki, T., and Fukuyama, S., Japan. Med. J., 4, 1951, 331-346; J. Antibiotics [Japan], 5, 1952, 469; Okami, Y. ibid. 477-480.)

Vegetative growth: In separate colonies with very thin yellow aerial mycelium. Submerged mycelium yellow or yellowish brown. Soluble pigment yellowish brown.

Aerial mycelium: Microscopically short, unbranched, bearing a straight conidia chain.

Nutrient agar: Isolated colonies; growth cream-colored, with triangular hole in the center. No aerial mycelium. Soluble pigment brown.

Gelation: Liquefaction crateriform. Growth cream-colored or brown; soluble pigment brown.

Milk: Growth slight, brown; aerial mycelium white; soluble pigment yellowish brown. Peptonization.

Starch: Hydrolysis.

Nitrate: No reduction.

Blood agar: Growth dark cream-yellow. Hemolysis strong.

Carrot: Growth slight, wrinkled, brownish cream-colored; aerial mycelium yellowish white. Pigment brown.

Egg: Growth greenish yellow, without aerial mycelium and with spreading reddish violet pigment.

Loeffler's coagulated serum: Growth cream-colored; no aerial mycelium. No hydrolysis. Pigment brown.

Antagonistic properties: Produces an antiviral agent, abikoviromycin.

Remarks: No tyrosinase produced. Culture utilizes glucose, maltose, and glycerol, but not arabinose, xylose, rhamnose, fructose, galactose, mannitol, sorbitol, lactose, sucrose, raffinose, and inulin.

71. Streptomyces odorifer (Rullman emend. Lachner-Sandoval) comb. nov. (Lachner-Sandoval, V., Ueber Strahlenpilze, Strassburg, 1898. Rullman, W. Centrbl. Bakt. I, 79, 1917, 383-390.)

Vegetative growth: Colorless, folded.

Aerial mycelium: Well-developed, white or light gray. Sporophores long, 
straight, branching. Spirals formed according to original report (none in our own observation). Spores spherical.

Synthetic agar: Growth cream-colored, with trace of brown. Aerial mycelium heavy cream-colored.

Nutrient agar: Growth folded, brown. Aerial mycelium white around edge. Faint soluble brown pigment.

Glucose agar: Growth cream-colored to brownish. Aerial mycelium abundant, cream-colored. Soluble pigment faint brownish.

Potato: Growth folded, brownish. Aerial mycelium cream-colored. Soluble pigment faint.

Gelatin: Surface ring cream-colored. Aerial mycelium thin, white. No soluble pigment. Liquefaction slow.

Milk: Surface ring colorless to brownish. No aerial mycelium. No coagulation. Peptonization.

Starch: Growth cream-colored to brown. Aerial mycelium abundant, cream-colored to straw-colored. No soluble pigment. Hydrolysis good.

Cellulose: Growth good.

Nutrient broth: Surface film colorless. Aerial mycelium heavy, creamcolored. No soluble pigment.

Nitrate: Reduction positive.

Sucrose: Inversion.

Paraffin, fats: Growth good.

Odor: Strong, characteristic of soil.

Antagonistic properties: Some strains give positive effects, others are negative.

72. Streptomyces roseoflavus Arai (Arai, T., J. Antibiotics [Japan], 4, 1951, 215-221.)

Vegetative growth: Colonies large, flat; vegetative mycelium limited to medium.

Aerial mycelium: White to rose-colored, producing numerous spirals. Spores oval to oblong, $0.8-1.0$ by $1.0-1.8 \mu$.

Synthetic agar: Growth penetrating deep into medium, colorless to yellowish; aerial mycelium powdery, white to yellow-rose.

Nutrient agar: Growth much-folded, white-gray to golden yellow; aerial mycelium, limited to center of colonies, white to rose-colored.

Glucose agar: Growth colorless to yellowish white; aerial mycelium rosecolored.

Potato: Growth yellow mass. No aerial mycelium; no soluble pigment. Gelatin: Liquefaction strong. Colonies at bottom of liquefied zone orangebrown. No soluble pigment. 
Milk: Ring cream-colored. Coagulation and peptonization rapid, medium becoming strongly alkaline.

Starch: Growth golden yellow; aerial mycelium whitish.

Cellulose: Growth on paper fair; cellulose decomposition.

Nitrate: Reduction positive.

Antagonistic properties: Produces a basic antibiotic, flavomycin, similar to neomycin.

Remarks: Culture similar to Streptomyces microflavus.

73. Streptomyces putrificans (Nicolaeva) comb. nov. (Nicolaeva, E., Arch. Biol. Nauk., 18, 1915, 229.)

Vegetative growth: Colorless.

Aerial mycelium: White.

Nutrient agar: Growth grayish, covered with white aerial mycelium. No soluble pigment.

Potato: Growth folded, sulfur-yellow. Aerial mycelium chalk-white. No soluble pigment.

Milk: Pellicle heavy, covered with white aerial mycelium. Peptonization gradual without previous coagulation.

Glucose broth: Soluble pigment yellow.

Loeffler's serum: Growth yellow. No aerial mycelium. Serum liquefied and colored yellowish brown.

Odor: Strong, putrefactive.

Habitat: Spring water.

Remarks: Decomposes proteins energetically, with the formation of bad smelling products $\left(\mathrm{H}_{2} \mathrm{~S}, \mathrm{NH}_{3}\right)$.

74. Streptomyces citreus (Krainsky) Waksman and Henrici. (Krainsky, A., Centrbl. Bakt. II, 41, 1914, 639-688.)

Aerial mycelium: Filaments with long, narrow open spirals. Conidia spherical to oval, $1.2-1.5$ by $1.2-1.8 \mu$.

Synthetic agar: Growth abundant, spreading, raised, wrinkled, citronyellow. Aerial mycelium covering surface, citron-yellow.

Nutrient agar: Growth restricted, cream-colored.

Glucose agar: Entire growth extensive, glossy, olive-yellow; center elevated.

Potato: Growth yellowish; aerial mycelium white.

Gelatin: Surface growth restricted, yellowish. Liquefaction in 35 days.

Milk: Surface growth cream-colored. Coagulation; peptonization, becoming alkaline. 
Starch: Growth abundant, yellowish green. Hydrolysis positive.

Glucose broth: Ring thin, wide, yellow; sediment flaky.

Nitrate: Reduction slight.

Temperature: Optimum $37^{\circ} \mathrm{C}$.

Antagonistic properties: Negative.

Remarks: The pigment formed is not soluble.

75. Streptomyces fulvissimus (Jensen) Waksman and Henrici. (Jensen, H. L., Soil Sci., 30, 1930, 66.)

Vegetative growth: Mycelium without any special characteristics.

Aerial mycelium: Hyphae short, straight, often trifurcated, 1.0-1.2 $\mu$ broad; no spiral formation; branches of hyphae break up into conidia, 1.01.2 by $1.2-1.5 \mu$.

Nutrient agar: Growth good; vegetative mycelium raised, finely wrinkled, deep red-brown; no aerial mycelium; pigment brownish yellow.

Potato: Growth good; vegetative mycelium raised, much wrinkled, rustbrown; aerial mycelium absent or traces of white; pigment gray to faint lemon-yellow.

Gelatin: Vegetative mycelium narrow, smooth, yellowish brown to redbrown; no aerial mycelium; no pigment; liquefaction complete in 10 to 12 days.

Czapek's agar: Growth good (one strain very scant); vegetative mycelium flat, narrow, first light golden, later deep orange to red-brown; aerial mycelium scant, sometimes almost absent, first white, later light grayish brown; pigment very characteristic, bright golden to orange.

Glycerol agar: Growth good; vegetative mycelium narrow, raised, smooth, golden to dark bronze; aerial mycelium scant, in patches, white to light cinnamon-brown; pigment intensely golden to orange.

Loeffler's blood serum: Vegetative mycelium red-brown; no aerial mycelium; pigment yellowish; no liquefaction.

Starch-casein agar: Growth good; vegetative mycelium spreading, folded, yellowish brown; aerial mycelium abundant, smooth, lead-gray; pigment dull yellow to orange.

Remarks: The characteristic golden pigment is formed in nearly all media in which the organism grows, but becomes most typical and attains its greatest brightness in synthetic agar media: it has indicator properties, turning red in strongly acid solutions. The species is easily recognized on agar plates by its bronze-colored colonies, surrounded by halos of bright yellow pigment.

76. Streptomyces chrysomallus Lindenbein. (Lindenbein, W., Arch. Mikrobiol., 17, 1952, 361-383.) 
Vegetative growth: Soft, on all media. Long, branched hyphae, with numerous staining granules.

Aerial mycelium: Hyphae long, sporulating, producing no spirals. Spores oval to elliptical, or spherical.

Synthetic agar: Growth good, crumb-like, light yellow. Aerial mycelium powdery, white; soluble pigment golden yellow.

Nutrient agar: Growth poor, shiny, golden yellow. Aerial mycelium thin, powdery. Soluble pigment golden yellow.

Glucose agar: Growth yellowish with tinge of orange. Aerial mycelium powdery, grayish white; soluble pigment light yellow to golden yellow.

Potato: Growth heavy, yellow, becoming brownish yellow or orange. Aerial mycelium cottony white to yellowish white.

Gelatin: Surface growth heavy, light to dark yellow. Aerial mycelium powdery, white. Liquefaction strong. Soluble pigment yellow-brown to deep brown in liquefied portion.

Milk: Growth abundant, colorless, reverse light yellow. Aerial mycelium cottony, snow-white becoming yellowish. Peptonization strong. Acidity slight.

Starch: Growth thin, colorless. Aerial mycelium powdery, white. Hydrolysis strong. No soluble pigment.

Glucose broth: Surface growth heavy yellowish; submerged flakes yellowish; soluble pigment golden yellow.

Cellulose: Growth poor.

Glucose-asparagine agar: Growth smooth, almost colorless to yellowish. Aerial mycelium powdery, white; soluble pigment faint yellow.

Calcium malate agar: Growth good, thin, smooth, colorless to faintly yellowish. Aerial mycelium powdery, grayish white.

Antagonistic properties: Produces actinomycin C (actinochrysin).

77. Streptomyces gougeroti (Duché) Waksman and Henrici. (Duché, J., Les actinomyces du groupe albus, P. Lechevalier, Paris, 1934.)

Aerial mycelium: Aerial hyphae short, gnarled. Spores oval.

Synthetic agar: Slow growth as punctiform colonies; cream-colored with smooth edge; no aerial mycelium; no soluble pigment.

Nutrient agar: Growth cream-colored with brownish reverse; soluble pigment faint yellowish. Aerial mycelium thin, white.

Glucose agar: Growth colorless to yellowish. Aerial mycelium thin, white. No soluble pigment.

Potato: Growth slow, greenish-tinged. Aerial mycelium thin, white. No soluble pigment.

Gelatin: Surface growth heavy, cream-colored. Aerial mycelium thin, white. No pigmentation. Liquefaction strong. 
Milk: Surface growth cream-colored. Aerial mycelium thin, white. Peptonization rapid.

Starch: Hydrolysis rapid.

Nutrient broth: Ring on surface cream-colored; flakes throughout medium. No soluble pigment. Aerial mycelium white.

Nitrate: No reduction.

Coagulated serum: Growth cream-colored, covered with white aerial mycelium; liquefaction rapid.

Synthetic solution: Submerged mycelium in form of flakes, later forming a surface pellicle; no soluble pigment.

Antagonistic properties: Active against fungi.

Remarks: This culture is believed to be intermediate between Streptomyces albus, with its abundant aerial mycelium, and Streptomyces almquisti, with its very scant aerial mycelium.

78. Streptomyces farinosus (Krassilnikov) comb. nov. (Krassilnikov, N. A., Actinomycetales, Akad. Nauk. USSR, Moskau, 1941, 51.)

Vegetative growth: Colonies colorless, smooth or rough.

Aerial mycelium: Powdery white on some media. Sporophores straight or wavy, singly or in clumps, forming no spirals. Organism produces segmented spores or oidiospores, cylindrical, $1.2-1.7$ by $0.6-0.8 \mu$. Some form oval or even spherical spores, produced by swelling of cylindrical forms.

Synthetic agar: Aerial mycelium poorly developed and spotty.

Potato: Growth colorless; aerial mycelium poorly developed.

Gelatin: Liquefaction fair to good.

Milk: Coagulation and rapid peptonization.

Starch: Hydrolysis rapid.

Cellulose: Growth of some strains.

Nitrate: Reduction fair.

Sucrose: Inversion of most strains, ready.

Antagonistic properties: None or very weak.

79. Streptomyces albidus (Duché) comb. nov. (Duché, J., Les actinomyces du groupe albus, P. Lechevalier, Paris, 1934.)

Aerial mycelium: Whitish but not snow-white. Sporophores long, open spirals. Spores spherical to oval. Soluble pigment produced in synthetic and other media.

Synthetic agar: Growth colorless; some drops of colorless guttation. Aerial mycelium white. Pigment yellowish.

Peptone agar: Growth colorless; aerial mycelium white; reverse slightly greenish; soluble pigment yellowish. 
Potato: Growth flat, colorless; aerial mycelium white. No soluble pigment.

Gelatin: Growth cream-colored. Liquefaction rapid. No soluble pigment.

Milk: Growth cream-colored. Coagulation weak; peptonization rapid; odor cheesy.

Starch: Hydrolysis good.

Cellulose: Growth good.

Nitrate: Reduction slow.

Odor: Strong, earthy.

Antagonistic properties: Strong.

Remarks: Closely related to Streptomyces albus; differs by more delicate growth, by a reverse that is often yellowish brown. Also related to Streptomyces microflavus, but differs from that described by Krainsky in that its growth is never rose-yellow and in its abundant growth on potato.

80. Streptomyces cinnamoneus Benedict, Pridham, and Lindenfelser. (Benedict, R. G., Pridham, T. G., and Lindenfelser, L. A., in press.)

Vegetative growth: Colonies large, with irregular margins.

Aerial mycelium: White, gradually changing to flesh color. No spirals. Spores globose, $0.6 \mu$ in diameter.

Synthetic agar: Growth colorless to white to cream-colored; aerial mycelium white to light cinnamon.

Nutrient agar: Growth cream to light lemon-yellow. No aerial mycelium.

Glucose agar: Growth colorless; light greenish yellow to dull yellowish orange in reverse; aerial mycelium white to cinnamon.

Potato: Growth grayish white to yellow-green to light brown. Aerial mycelium light gray to gray. No soluble pigment.

Gelatin: Growth white, flocculent; no aerial mycelium. Liquefaction rapid. No soluble pigment.

Milk: Ring light brown; aerial mycelium limited, white. Peptonization rapid, with alkaline reaction.

Starch: Growth colorless to brownish; aerial mycelium white. Hydrolysis.

Glucose broth: Ring white to lime-green. No aerial mycelium.

Nitrate: No reduction.

Carrot: Growth white to cream-colored. Aerial mycelium cretaceous. No soluble pigment.

Oatmeal agar: Growth tough, leathery, yellowish green to cream-yellow. Aerial mycelium floccose, pale violet to faint cinnamon. Exudate tan to white.

Temperature: Good growth at $25^{\circ}-37^{\circ} \mathrm{C}$.

Antagonistic properties: Produces cinnamycin, a polypeptide antibiotic.

Source: Japanese soil. 
Remarks: Resembles Streptomyces griseocarneus. Utilizes xylose, fructose, inositol, starch, dextrin, galactose, and maltose, but not arabinose, rhamnose, dulcitol, and salicin.

81. Streptomyces violaceoniger (Waksman and Curtis) Waksman and Henrici. (Waksman, S. A., and Curtis, R. E., Soil Sci., 1, 1916, 111.)

Synthetic agar: Colony at first dark gray, turning almost black, 2-4 $\mathrm{mm}$ in diameter. Surface glossy, much-folded, with very thin, gray margin. Aerial mycelium white to gray after the colony is well developed. Pigment bluish black at a later stage of growth. The pigment slowly dissolves in the medium, turning almost black. Odor fairly strong. Microscopically two types of mycelium are found: the thin, branching filaments of the substratum, and the thick filaments of the aerial mycelium. The aerial mycelium fragments not very rapidly, producing a few conidia, spherical and oval, $1.2-1.5$ by $1.2-2.3 \mu$. These often occur in chains.

Potato: Growth at first very slight, but after 48 hours develops into continuous, thick yellowish gray smear which later turns brown, with white aerial mycelium covering the growth. Medium not colored.

Gelatin: Growth gray; no aerial mycelium. Liquefaction rapid around colony, but with no change in color.

Synthetic solution: Colonies large, $2-3 \mathrm{~mm}$ in diameter, appearing at the bottom and surface of the solution, but none throughout the medium. Colonies bluish, with a regular margin. Medium not colored.

Antagonistic properties: None; certain strains show activity.

82. Streptomyces gedanensis (Löhlein) comb. nov. (Löhlein, M., Z. Hyg., 63, 1909, 1-16.)

Aerial mycelium: Hyphae short, gnarled; spores short, oval to spherical. Synthetic agar: Growth dark to almost black, with dark reverse. Aerial mycelium abundant, mouse-gray. No soluble pigment.

Nutrient agar: Growth thin, colorless. No aerial mycelium. No soluble pigment.

Glucose agar: Growth cream-colored, becoming black with light margin. Aerial mycelium abundant, mouse-gray.

Potato: Growth lichnoid, cream-colored to brownish. No aerial mycelium. No soluble pigment.

Gelatin: Growth thin, flaky. No soluble pigment. Liquefaction rapid. Milk: Surface ring cream-colored. No peptonization.

Starch: Growth yellowish to cream-colored. Aerial mycelium light gray. Hydrolysis strong.

Nutrient broth: Growth on bottom of tube flaky. 
Nitrate: No reduction.

Source: Sputum of patient with chronic lung disease.

83. Streptomyces griseus (Krainsky emend. Waksman et al.) Waksman et al. (Krainsky, A., Centrbl. Bakt. II, 41, 1914, 639-688.)

Vegetative growth: Colonies smooth or folded, colorless, later turning olive-buff.

Aerial mycelium: Abundant, powdery, water-green. Sporophores produced in tufts (original Krainsky and Krassilnikov report, spiral formation). Spores spherical to oval, 0.8 by $0.8-1.7 \mu$.

Synthetic agar: Growth thin, spreading, colorless, becoming olive-buff. Aerial mycelium thick, powdery, water-green.

Nutrient agar: Growth abundant, almost transparent, cream-colored; aerial mycelium powdery, white to light gray. No soluble pigment.

Glucose agar: Growth elevated in center, radiate, cream-colored to orange, erose margin.

Potato: Growth wrinkled yellowish to brownish, covered with white, powdery aerial mycelium.

Gelatin: Surface growth greenish yellow or cream-colored with brownish tinge. Liquefaction rapid.

Milk: Ring cream-colored; coagulation with rapid peptonization, becoming alkaline.

Starch: Growth thin, spreading, transparent. Hydrolysis strong.

Glucose broth: Pellicle abundant, much-folded, yellowish with greenish tinge.

Nitrate: Reduction positive.

Temperature: Optimum $37^{\circ} \mathrm{C}$.

Antagonistic properties: Strongly antagonistic. Different strains of this organism produce different antibiotics. One of these, streptomycin, is active against a large number of bacteria and actinomycetes, but not against fungi and viruses. Some strains produce grisein. Others form candicidin.

Habitat: Soils, river muds, throat of chicken.

Remarks: The foregoing description is based largely on the culture isolated in 1915 by Waksman and Curtis. It is the latter description which was used for identifying the streptomycin-producing strain. This strain shows in its mycelium no spirals but tuft-like masses of sporulating hyphae. (See Waksman, S. A., Reilly, H. C., and Harris, D. A., J. Bacteriol., 56, 1948, 259-269.)

84. Streptomyces longissimus (Krassilnikov) comb. nov. (Krassilnikov, N. A., Actinomycetales, Akad. Nauk. USSR, Moskau, 1941, 38.) 
Vegetative growth: Colonies pigmented bright orange or golden red. Pigment not dissolved in medium.

Aerial mycelium: Weakly developed; totally absent on some media. Sporophores straight, short, single or branched. Spores elongated 1.0-1.3 by $0.7 \mu$, cylindrical.

Synthetic agar: Aerial mycelium well developed, velvety, white-yellow.

Potato: Aerial mycelium well developed, of characteristic color.

Gelatin: Liquefaction rapid.

Starch: Hydrolysis rapid.

Cellulose: Growth good.

Paraffin and waxes: Growth good.

Antagonistic properties: Weakly antagonistic.

Remarks: Similar to Streptomyces fradiae.

85. Streptomyces olivaceus (Waksman) Waksman and Henrici. (Waksman, S. A., Soil Sci., 7, 1919, 117.)

Aerial mycelium: Clumps small, with straight and branching hyphae. No spirals on most media. Conidia spherical and oval, $0.9-1.1$ by $0.9-2.0 \mu$.

Synthetic agar: Growth abundant, spreading, developing deep into medium, yellow to olive-ocher, reverse yellow to almost black. Aerial mycelium mouse-gray to light drab.

Nutrient agar: Growth white, glistening.

Glucose agar: Growth abundant, restricted, entire, center raised.

Potato: Growth abundant, much-wrinkled, elevated, gray, turning sulfuryellow on edge.

Gelatin: Liquefaction; sediment cream-colored, flaky, yellow.

Milk: Growth faint, pinkish; coagulation; peptonization, becoming alkaline.

Starch: Growth thin, spreading, yellowish green. Hydrolysis.

Glucose broth: Ring sulfur-yellow.

Nitrate: Reduction to nitrite.

Temperature: Optimum $25^{\circ} \mathrm{C}$.

Antagonistic properties: Positive.

Remarks: The pigment formed is not soluble.

86. Streptomyces microflavus (Krainsky) Waksman and Henrici. (Krainsky, A., Centrbl. Bakt. II, 41, 1914, 639-688.)

Aerial mycelium: Well-developed, cottony, white. Spores spherical to rod-shaped, often in pairs or chains, 2.0 by $2.0-5.0 \mu$.

Nutrient agar: Colonies yellow; aerial mycelium in 3 to 4 weeks, yellowish rose. 
Glucose agar: Aerial mycelium in about 12 days, rose-yellow.

Potato: Growth yellowish. No aerial mycelium.

Gelatin: Colonies small, yellowish. Liquefaction rapid.

Milk: Coagulation and peptonization rapid.

Starch: Diastatic action strong.

Cellulose: Growth scant.

Glucose broth: Colonies in depth small, spherical.

Nitrate: Reduction to nitrite.

Calcium-malate agar: Colonies minute, yellow. No aerial mycelium.

Temperature: Optimum $25^{\circ} \mathrm{C}$.

Antagonistic properties: Positive.

87. Streptomyces cacaoi (Waksman) Waksman and Henrici. (Waksman, in Bunting, Ann. Appl. Biol., 19, 1932, 515.)

Aerial mycelium: Long with considerable spiral formation; spirals long and open, not compact.

Synthetic agar: Growth thin, yellowish, later turning reddish brown; no soluble pigment; aerial mycelium light gray to mouse-gray, with white edge. Typical odor of streptomyces.

Nutrient agar: Growth brown-colored, covered with tiny patches of ivory-colored aerial mycelium.

Glucose agar: Same as on synthetic agar.

Potato: Growth abundant, brownish; aerial mycelium white to mousegray.

Gelatin: Growth flocculent. No aerial mycelium. Liquefaction rapid. No pigment production.

Nitrate: Reduction limited.

Antagonistic properties: Certain strains produce an antibiotic designated as cacaomycetin.

Source: Three strains isolated from cacao beans in Nigeria. There were slight differences among the three strains; the foregoing description is of Strain I.

Remarks: Strong proteolytic enzymes acting on casein; strong diastatic action, no sugar or dextrin left in 1 per cent starch solution after a few days.

88. Streptomyces novaecaesareae (Waksman and Curtis) Waksman and Henrici. (Waksman, S. A., and Curtis, R. E., Soil Sci., 1, 1916, 111.)

Aerial mycelium: Filaments with both straight and spiral aerial hyphae; spirals dextrorse. Conidia oval to elongate.

Synthetic agar: Growth gray, becoming bluish, glossy, much-wrinkled. Aerial mycelium appears late; white.

Nutrient agar: Growth thin, cream-colored. 
Glucose agar: Growth restricted, gray, becoming red.

Potato: Growth wrinkled, cream-colored, turning yellowish.

Gelatin: Surface colonies small, cream-colored. Liquefaction slow.

Milk: Ring gray; coagulation; peptonization slow, becoming faintly alkaline.

Starch: Colonies restricted, circular, bluish violet. Hydrolysis positive.

Glucose broth: Sediment fine, flaky, colorless.

Nitrate: Reduction to nitrite.

Temperature: Optimum $37^{\circ} \mathrm{C}$.

Antagonistic properties: Negative.

Remarks: Soluble purple pigment formed.

89. Streptomyces exfoliatus (Waksman and Curtis) Waksman and Henrici. (Waksman, S. A., and Curtis, R. E., Soil Sci., 1, 1916, 116.)

Aerial mycelium: Filaments slightly wavy with tendency to form spirals. Conidia oval, $1.0-1.5$ by $1.2-1.8 \mu$.

Synthetic agar: Growth smooth, glossy, colorless, becoming brown. Aerial mycelium in white patches over surface.

Nutrient agar: Growth only in depth of medium.

Potato: Growth somewhat wrinkled, gray, becoming brown.

Gelatin: Surface growth cream-colored. Liquefaction.

Milk: Ring cream-colored; soft coagulation in 12 days; peptonization slow.

Starch: Growth restricted, gray becoming brown. Hydrolysis.

Glucose broth: Colonies in depth small, grayish.

Nitrate: Reduction to nitrite.

Temperature: Optimum $37^{\circ} \mathrm{C}$.

Antagonistic properties: Positive.

Remarks: Brown, soluble pigment formed.

90. Streptomyces gelaticus (Waksman) Waksman and Henrici. (Waksman, S. A., Soil Sci., 8, 1919, 165.)

Aerial mycelium: Branching, with open spirals.

Synthetic agar: Growth colorless, spreading, chiefly deep into the medium. Aerial mycelium thin, white, turning grayish.

Nutrient agar: Growth only on surface, wrinkled, cream-colored.

Glucose agar: Growth abundant, spreading, white.

Potato: Growth abundant, much-wrinkled, greenish, becoming black with yellowish margin.

Gelatin: Liquefaction; sediment flaky, cream-colored.

Milk: Ring pinkish; coagulation; peptonization with distinctly alkaline reaction. 
Starch: Growth thin, spreading, cream-colored. Hydrolysis positive. Glucose broth: Pellicle thin, cream-colored; sediment slight, flaky.

Nitrate: Reduction to nitrite slight.

Temperature: Optimum $25^{\circ} \mathrm{C}$.

Antagonistic properties: Positive; some strains show no actjvity.

Remarks: Soluble brown pigment formed.

91. Streptomyces glaucus (Lehmann and Schütze emend. Krassilnikov) comb. nov. (Krassilnikov, N. A., Actinomycetales, Akad. Nauk. USSR, Moskau, 1941, 46.)

Aerial mycelium: Well-developed, cottony, at first white then turning green, similar to green penicillia. Sporophores form compact spirals with 3-5 turns. Spores oval to spherical, 1.0 by $0.8 \mu$.

Synthetic agar: Growth colorless; soluble pigment brown.

Nutrient agar: Growth heavy, covered with green aerial mycelium.

Potato: Growth heavy, covered with velvety, green aerial mycelium.

Gelatin: Liquefaction slow.

Milk: Peptonization slow, with prior coagulation by some strains.

Starch: Hydrolysis rapid.

Cellulose: Growth good.

Nitrate: Reduction.

Sucrose: Inversion poor.

Paraffin: Growth good.

Antagonistic properties: All strains strongly antagonistic.

92. Streptomyces rutgersensis (Waksman and Curtis) Waksman and Henrici. (Waksman, S. A., and Curtis, R. E., Soil Sci., 1, 1916, 123.)

Aerial mycelium: Filaments branching, with abundant open and closed spirals; hyphae fine, long, branching. Conidia spherical and oval, 1.0-1.2 $\mu$, with tendency to bipolar staining.

Synthetic agar: Growth thin, spreading, colorless, becoming brownish to almost black. Aerial mycelium thin, white, becoming dull-gray.

Nutrient agar: Growth thin, wrinkled, cream-colored.

Glucose agar: Mycelium abundant, brown, becoming black with creamcolored margin.

Potato: Growth abundant, much-folded, white-gray.

Gelatin: Surface growth spreading, cream-colored. Liquefaction.

Milk: Ring cream-colored; coagulation; slow peptonization, becoming alkaline.

Starch: Growth gray, spreading. Hydrolysis.

Nitrate: Reduction to nitrite.

Temperature: Optimum $37^{\circ} \mathrm{C}$. 
Antagonistic properties: Limited.

Remarks: The pigment formed is not soluble.

93. Streptomyces halstedii (Waksman and Curtis) Waksman and Henrici. (Waksman, S. A., and Curtis, R. E., Soil Sci., 1, 1916, 124.)

Aerial mycelium: Mycelium branching; hyphae with close spirals. Conidia oval or rod-shaped, $1.0-1.2$ by $1.2-1.8 \mu$.

Synthetic agar: Growth abundant, heavy, spreading, raised, light, becoming dark, almost black. Aerial mycelium white, turning dull-gray.

Nutrient agar: Growth restricted, wrinkled, cream-colored.

Glucose agar: Growth spreading, colorless, wrinkled, center elevated, edge lichnoid, becoming brown.

Potato: Growth abundant, moist, wrinkled, cream-colored with green tinge.

Gelatin: Liquefaction; small, cream-colored masses in bottom of tube.

Milk: Ring cream-colored; coagulation; peptonization, becoming alkaline.

Starch: Growth abundant, glossy, brownish. Hydrolysis.

Glucose broth: Colonies in bottom of tube small, colorless.

Nitrate: Reduction to nitrite.

Temperature: Optimum $37^{\circ} \mathrm{C}$.

Antagonistic properties: Strongly antagonistic; some strains show only antifungal activity; some strains produce carbomycin.

Remarks: The pigment formed is not soluble.

94. Streptomyces fumosus (Krassilnikov) comb. nov. (Krassilnikov, N. A., Actinomycetales, Akad. Nauk. USSR, Moskau, 1941, 58.)

Aerial mycelium: Sporophores straight; spores cylindrical, $1.5-2.0$ by $0.7 \mu$, later round.

Synthetic agar: Growth dark brown, pigment insoluble; aerial mycelium well developed, cottony, dusty-colored, occasionally gray-white.

Nutrient agar: Growth dark brown, aerial mycelium white; medium brown-colored.

Potato: No growth, or only faint dark gray aerial mycelium.

Gelatin: Liquefaction medium.

Milk: No coagulation; liquefaction slow, with production of dark brown to almost black pigment.

Starch: Hydrolysis good.

Cellulose: No growth.

Sucrose: Inversion weak.

Antagonistic properties: None. 
95. Streptomyces lipmanii (Waksman and Curtis) Waksman and Henrici. (Waksman, S. A., and Curtis, R. E., Soil Sci., 1, 1916, 123.)

Aerial mycelium: Mycelium and hyphae straight, branching. Conidia oval, $0.8-1.1$ by $1.0-1.5 \mu$.

Synthetic agar: Growth abundant, raised, colorless, becoming light brown and wrinkled. Aerial mycelium white, turning gray.

Nutrient agar: Growth radiately wrinkled, glossy, yellow.

Glucose agar: Growth irregular, spreading, light yellow.

Potato: Growth abundant, wrinkled, cream-colored.

Gelatin: Liquefaction, with cream-colored, flaky sediment.

Milk: Ring cream-colored; coagulation; peptonization with alkaline reaction.

Starch: Growth transparent, becoming dark with age. Hydrolysis positive.

Glucose broth: Ring white, with abundant, colorless, flaky sediment.

Nitrate: Reduction to nitrite.

Temperature: Optimum $25^{\circ} \mathrm{C}$.

Antagonistic properties: Good, though some strains show no activity.

Remarks: The pigment formed is not soluble.

96. Streptomyces hygroscopicus (Jensen) Waksman and Henrici. (Jensen, H. L., Proc. Linnean Soc. N. S. Wales, 56, 1931, 359.)

Vegetative growth: Hyphae $0.6-0.8 \mu$ in diameter.

Aerial mycelium: Hyphae long, tangled, branched, $0.8-1.0 \mu$ in diameter. Spirals numerous, sinistrorse, narrow, usually short, only 1 or 2 turns, closed, typically situated as dense clusters on the main stems of the aerial hyphae. Spores oval, $0.8-1.0$ by $1.0-1.2 \mu$.

Synthetic agar: Growth good, folded, glossy surface; white to creamcolored, later sulfur-yellow to yellowish gray, with golden to light orange reverse. Soluble pigment of the same color. Aerial mycelium scant, thin, white, or absent.

Nutrient agar: Growth good, raised, wrinkled, glossy, cream-colored; later yellowish gray with yellowish brown reverse. Occasionally, a scant white aerial mycelium.

Glucose agar: Growth good; granulated, cream-colored to straw-yellow, later dull chrome-yellow to brownish orange. Aerial mycelium thin, smooth, dusty, white to pale yellowish gray; later interspersed with small, moist, dark violet-gray to brownish patches which gradually spread over the whole surface. Soluble pigment light yellow. 
Potato: Growth fair, raised, wrinkled, cream-colored, later yellowish gray to dull brownish. Aerial mycelium absent or trace of white.

Gelatin: Liquefaction slow. No pigment produced.

Milk: Peptonization complete in 3 to 4 weeks at $30^{\circ} \mathrm{C}$, without any previous coagulation. The reaction becomes faintly acid ( $\mathrm{pH} 6.0$ or less).

Starch: Hydrolysis.

Cellulose: Decomposed readily by some strains.

Nitrate: No reduction with sucrose as source of energy.

Sucrose: Inversion.

Antagonistic properties: Positive.

Remarks: Aerial mycelium on certain media (glucose or glycerol-asparagine agar) becomes moistened and exhibits dark, glistening patches. These patches, when touched with a needle, prove to be a moist, smeary mass of spores. This characteristic feature is not equally distinct in all strains.

97. Streptomyces longisporoflavus (Krassilnikov) comb. nov. (Krassilnikov, N. A., Actinomycetales, Akad. Nauk. USSR, Moskau, 1941, 30.)

Vegetative growth: Colonies yellow, lemon-yellow or dirty yellow, seldom golden yellow. Pigment insoluble in medium.

Aerial mycelium: Well-developed, velvety, whitish yellow to brownish yellow. Sporophores produce long open spirals. Spores cylindrical or elongated, $1.0-1.5$ by $0.7 \mu$; some with rounded ends and swollen in center. Gelatin: Liquefaction medium, 10-15 days.

Milk: Coagulation; peptonization slow.

Starch: Hydrolysis weak.

Cellulose: No growth.

Nitrate: Reduction strong.

Sucrose: No inversion.

Antagonistic properties: Weakly antagonistic.

Remarks: Some strains have a greenish tinge, depending on composition of medium.

98. Streptomyces candidus (Krassilnikov) comb. nov. (Not Streptothrix candida Petruschky.) (Krassilnikov, N. A., Actinomycetales, Akad. Nauk. USSR, Moskau, 1941, 49.)

Vegetative growth: Organism grows well on various media.

Aerial mycelium: Sporophores long, straight or wavy, but never forming spirals. Spores cylindrical, elongated, $1.5-2.0$ by $0.7 \mu$; on maturing, some become more rounded.

Synthetic agar: Colonies well-developed, colorless, not pigmenting medium. Aerial mycelium cottony white. 
Nutrient agar: Growth good, lichnoid or smooth; aerial mycelium whitish.

Gelatin: Liquefaction slow.

Milk: Coagulation and peptonization weak.

Starch: Hydrolysis rapid.

Cellulose: Growth good.

Nitrate: Reduction positive.

Sucrose: Inversion.

Antagonistic properties: Weak.

99. Streptomyces alboflavus (Waksman and Curtis) Waksman and Henrici. (Waksman, S. A., and Curtis, R. E., Soil Sci., 1, 1916, 99-134.)

Aerial mycelium: Straight, branching, with very little tendency to form spirals; very few oval-shaped spores formed.

Synthetic agar: Growth glossy, spreading, colorless, becoming yellowish. Aerial mycelium white, powdery, with yellow tinge.

Nutrient agar: Growth restricted, cream-colored; no soluble pigment.

Glucose agar: Growth restricted, much-folded, cream-colored with sulfuryellow surface; no soluble pigment.

Potato: Growth wrinkled, moist, cream-colored.

Gelatin: Surface growth abundant, colorless. Liquefaction slow, occurring in 35 days.

Milk: Surface ring pinkish. No coagulation. Peptonization, becoming alkaline.

Starch: Growth thin, spreading, yellowish. Hydrolysis.

Glucose broth: Colonies on surface cylindrical, white; later flaky mass in bottom of tube.

Nitrate: Reduction to nitrite.

Temperature: Optimum $37^{\circ} \mathrm{C}$.

Antagonistic properties: Positive.

Remarks: The pigment formed is not soluble.

100. Streptomyces flocculus (Duché) Waksman and Henrici. (Duché, J., Les actinomyces du groupe albus, P. Lechevalier, Paris, 1934.)

Vegetative growth: Velvety surface with cottony or floccose edge.

Synthetic agar: Growth cream-colored, later covered with white aerial mycelium; no soluble pigment.

Nutrient agar: Growth cream-colored, later covered with white aerial mycelium; no soluble pigment.

Potato: Growth punctiform, covered with white aerial mycelium; pigment faint, yellowish.

Gelatin: Growth limited. Liquefaction slow. 
Milk: Growth rose-colored; peptonization slow.

Coagulated serum: Growth cream-colored; aerial mycelium fine, white; liquefaction slow.

Glucose-asparagine agar: Colonies limited, cream-colored, hardly raised above the surface of the medium; occasionally abundant growth produced with white aerial mycelium, colorless on reverse side.

Glucose-asparagine solution: Branching immersed filaments, $0.8 \mu$ in diameter; aerial mycelium 1.0 by $1.2 \mu$ with numerous spores; flakes settle to bottom of tube.

Peptone solution: Surface colonies cream-colored.

Tyrosine medium: Growth whitish; no pigment.

101. Streptomyces sp. Leben, Stessel, and Keitt. (Leben, C., Stessel, G. J., Keitt. G. W., Mycologia, 44, 1952, 159-169.)

Vegetative growth: On most media early growth at first white and later gray; the color change appears to be associated with the development of dense masses of spores.

Aerial mycelium: Spirals situated typically in dense groups along the main portions of aerial hyphae, especially toward the center of the colony. In early formation of sporing hyphae, spirals tend to be open; they become closed and compact, however, prior to the formation of spores. In late stages these fruiting structures tend to appear ball-like rather than spiral. Spores oval, hyaline, and $0.6-1.3 \mu$ wide by $0.7-2.0 \mu$ long; diameter of spore spirals $4-8 \mu$.

Synthetic agar: Aerial mycelium abundant, pebbly, light neutral gray. No diffusible pigment.

Potato: Vegetative growth finely wrinkled, cream-colored with sparse, white, aerial mycelium. Plug darkened slightly.

Gelatin: Vegetative mycelium translucent, cream-colored; aerial mycelium sparse, white. Liquefaction slight at 15 days, moderate at 30 days. No diffusible pigment.

Milk: Growth ring cream-colored. Coagulation; peptonization in 15 to 30 days, becoming slightly alkaline. Serum yellowish orange, slightly turbid.

Starch: Aerial mycelium abundant, pebbly white to neutral gray. No diffusible pigment. Diastatic action weak to moderate.

Glucose broth: Surface colonies coalescing, white, powdery. Liquid clear, not pigmented. Small amount of flaky sediment present.

Nitrate: Reduction to nitrite slight.

Glucose-asparagine agar: Results the same as on synthetic agar.

Glucose-peptone agar: Results the same as on synthetic agar.

Malate-glycerin agar: Aerial mycelium abundant, pebbly, light neutral gray. Diffusible pigment faint green. 
Nutrient peptone agar: Vegetative mycelium shiny cream-colored; aerial mycelium moderate, pebbly, white. No diffusible pigment.

Potato-glucose agar: Aerial mycelium abundant, pebbly, neutral gray. Diffusible pigment faint, brown.

Yeast-extract agar: Aerial mycelium abundant, pebbly, neutral gray. No diffusible pigment.

Antagonistic properties: Produces an antifungal agent, helixin.

102. Streptomyces fradiae (Waksman and Curtis) Waksman and Henrici. (Waksman, S. A., and Curtis, R. E., Soil Sci., 1, 1916, 99134.)

Aerial mycelium: Filaments and hyphae straight, branching; no spirals. Spores rod- or oval-shaped, 0.5 by $0.7-1.25 \mu$.

Synthetic agar: Growth smooth, spreading, colorless. Aerial mycelium thick, cottony mass covering surface, seashell-pink.

Nutrient agar: Growth restricted yellowish, becoming orange-yellow; no soluble pigment.

Glucose agar: Growth restricted, glossy, buff-colored, lichnoid margin.

Potato: Growth restricted, orange-colored.

Gelatin: Growth dense, cream-colored to brownish.

Milk: Ring faint, cream-colored; coagulation and peptonization.

Starch: Growth spreading, colorless. Hydrolysis positive.

Glucose broth: Ring dense, narrow, orange-colored; sediment abundant, flaky, colorless.

Nitrate: No reduction.

Temperature: Optimum $25^{\circ} \mathrm{C}$.

Antrgonistic properties: Highly antagonistic. Produces an antibacterial agent, neomycin, and an antifungal agent, fradicin.

103. Streptomyces albosporeus (Krainsky) Waksman and Henrici. (Krainsky, A., Centrbl. Bakt. II, 41, 1914, 639-688.)

Aerial mycelium: Hyphae straight, branching, with occasional spirals. Spores spherical or oval, $0.8-1.2$ by $1.0-1.8 \mu$.

Synthetic agar: Growth spreading, colorless, with pink center, becoming brownish. Aerial mycelium white, covering the whole surface. No soluble pigment.

Nutrient agar: Colonies minute, cream-colored.

Glucose agar: Growth spreading, wrinkled, radiate, entire, red.

Potato: Growth thin, spreading, wrinkled, gray, becoming brown with greenish tinge. 
Gelatin: Growth yellow, changing to red, with hyaline margin. Liquefaction slow.

Milk: Ring scant, pink. No coagulation. No peptonization.

Starch: Growth thin, spreading, transparent, with red tinge. Hydrolysis ready.

Glucose broth: Surface ring pinkish.

Nitrate: Reduction to nitrite.

Temperature: Optimum $37^{\circ} \mathrm{C}$.

Antagonistic properties: Positive.

104. Streptomyces purpurascens Lindenbein. (Lindenbein, W., Arch. Mikrobiol., 17, 1952, 361-383.)

Vegetative growth: Usually heavy, red to carmine-red to almost purple.

Aerial mycelium: Cottony, white. Spirals observed in 6 days, but they may be lacking altogether.

Synthetic agar: Growth typical carmine-red. Aerial mycelium cottony, chalk-white. Soluble pigment brown-red.

Nutrient agar: Growth light brown, with dark brown reverse. Aerial mycelium velvety, gray.

Glucose agar: Growth lichnoid, red to red-brown. Aerial mycelium cottony, chalk-white. Soluble pigment light brown.

Potato: Growth very good, brownish to reddish. Aerial mycelium velvety, white. No soluble pigment.

Gelatin: Surface growth heavy, light-brown. Aerial mycelium cottony, white. Soluble pigment red-brown. Liquefaction rapid.

Milk: Growth lichnoid, red to dark brown. Aerial mycelium velvety to cottony, white. No proteolysis.

Starch: Growth lichnoid, light-carmine-colored. Aerial mycelium white. No soluble pigment. Hydrolysis strong.

Cellulose: Growth very good, white to red.

Glucose broth: Pellicle heavy, with limited bottom growth, carmine-red later copper-red. Aerial mycelium powdery, chalk-white. Soluble pigment carmine-red.

Glucose-asparagine agar: Growth carmine-red. Aerial mycelium cottony, white. Soluble pigment orange.

Calcium malate agar: Growth carmine-red. Aerial mycelium chalk-white. Soluble pigment brick-red.

Antagonistic properties: Produces rhodomycin.

Remarks: On continued growth on synthetic media the culture may lose the property to produce the typical pigment. It can be regained, however, by growth on organic media. 
105. Streptomyces longispororuber (Krassilnikov) comb. nov. (Krassilnikov, N. A., Actinomycetales, Akad. Nauk. USSR, Moskau, 1941, 22.)

Vegetative growth: Colonies pigmented dark red to purple-red to light rose. Little pigment dissolves into the media, except those containing fat. Certain strains secrete a soluble brown substance in protein-containing media.

Aerial mycelium: Weakly developed, whitish to rose-white; sporophores in some strains well-developed, velvety, long, straight, seldom forming open spirals. Spores cylindrical to elongated, $1.0-1.5$ by $0.7 \mu$, with sharply cut ends; a few are slightly swollen, becoming oval or spherical, $1 \mu$ in diameter.

Synthetic agar: Growth light red to pale rose; pigment insoluble in medium.

Gelatin: Liquefaction medium.

Milk: Coagulation weak; peptonization fair to good.

Starch: Hydrolysis slow to good.

Cellulose: No growth of some strains; others make some growth.

Nitrate: Reduction positive for some strains.

Antagonistic properties: Strongly antagonistic to a number of bacteria.

Remarks: Related to Streptomyces ruber, except for the cylindrical spores. Very variable, especially as regards production of aerial mycelium.

106. Streptomyces oidiosporus (Krassilnikov) comb. nov. (Krassilnikov, N. A., Actinomycetales, Akad. Nauk. USSR, Moskau, 1941, 23.)

Vegetative growth: Colonies red and rose to pale. Pigment insoluble in medium.

Aerial mycelium: Poorly developed, covering medium with velvety rosewhite color. Sporophores straight or wavy, never forming spirals; they are short or long, frequently forming broom-shaped structures. Oidiospores produced by segmentation of sporophores. Spores $1.0-1.8$ by $1.0 \mu$, frequently appearing as double cocci.

Gelatin: Aerial mycelium weakly developed, frequently lacking; hyphae short, covering colony as rose-white velvet; frequently occurs in spots or is absent. Liquefaction weak.

Milk: No coagulation; peptonization medium.

Starch: Hydrolysis rapid.

Cellulose: No growth.

Nitrate: Reduction positive.

Antagonistic properties: None.

Remarks: Cultures resemble Streptomyces ruber and Streptomyces longispororuber. Some strains were obtained as variants of Nocardia rubra. 
107. Streptomyces melanocyclus (Merker) Waksman and Henrici. (Merker, Centrbl. Bakt. II, 31, 1911, 589.)

Vegetative growth: Much-folded.

Aerial mycelium: Dark brown. Spores spherical, $0.9 \mu$ in diameter. Produces red pigment, as well as soluble brown substance, as a result of which culture and substrate become red-brown to almost black with a shade of red.

Gelatin: Growth poor. Liquefaction rapid.

Milk: Coagulation; peptonization rapid.

Starch: Hydrolysis good.

Cellulose: Decomposition.

Nutrient broth: Colonies colorless, spherical.

Nitrate: Reduction to nitrite.

Sucrose: Inversion.

Calcium malate agar: Colonies small, flat, orange-red. Aerial mycelium black, occurring along the edges.

Antagonistic properties: Strong effect upon various bacteria; some strains show no activity.

108. Streptomyces acidophilus (Jensen) Waksman and Henrici. (Jensen, H. L., Soil Sci., 25, 1928, 226.)

Vegetative growth: Mycelium profusely branched, hyphae $0.6-0.8 \mu$ in diameter with homogeneous protoplasm and no visible septa.

Aerial mycelium: Hyphae 1.0-1.2 $\mu$ in diameter, somewhat branched, forming either very few or very numerous sinistrorse spirals. Oval conidia $1.0-1.2$ by $1.2-1.5 \mu$.

Nutrient agar: No growth.

Glucose agar: Growth at $25^{\circ} \mathrm{C}$ good. Substratum mycelium raised, somewhat wrinkled, colorless in young cultures. Aerial mycelium thin, white at first, later gray or yellowish brown.

Potato: Growth good, raised, folded. No discoloration.

Gelatin: Growth after 10 days very scant, thin, semitransparent, colorless. Liquefaction slow.

Milk: No growth.

Starch: Growth at $25^{\circ} \mathrm{C}$ good. Substratum mycelium flat, smooth, colorless. Aerial mycelium abundant, smooth, white. Diastatic action.

Nutrient broth: No growth.

Nitrate: Reduction only a trace in two strains.

Sucrose: No inversion.

Czapek's agar: No growth.

Antagonistic properties: Strongly positive.

Remarks: Distinctive character: has the ability to live in acid media only. 
109. Streptomyces rubescens (Jarach) Umezawa, Tazaki, and Fukuyama. (Umezawa, H., Tazaki, T., and Fukuyama, S., Boll. Sez. Ital. Soc. Intern. Microbiol., 3, 1931, 43; J. Antibiotics [Japan], 5, 1952, 469.)

Aerial mycelium: Powdery, white, Microscopically aerial mycelium short, curved, well-branched, bearing spherical or oval conidia in chain. No spirals.

Synthetic agar: Submerged mycelium at first white, changing to salmonpink after about 10 days' incubation. No soluble pigment; reverse of growth changes to salmon-pink.

Nutrient agar: Same as on synthetic agar.

Potato: Growth coral-pink; aerial mycelium powdery, white. Plug changes slightly to brown. No soluble pigment.

Gelatin: Surface growth coral-pink. No liquefaction and no pigmentation.

Milk: Growth coral-pink; aerial mycelium powdery, white. No coagulation and no digestion. Sometimes slightly reddish soluble pigment.

Starch: No hydrolysis.

Glucose broth: Surface growth with white powdery aerial mycelium becomes salmon-pink or coral-pink. The liquid later becomes coral-red.

Nitrate: No reduction to nitrite.

Blood agar: After 10 days' incubation, mycelium in the agar becomes salmon-pink and bears powdery white aerial mycelium. No soluble pigment. No hemolysis.

Carrot: Growth dark reddish orange; aerial mycelium powdery, white. Plug changes very slightly to dark color.

Egg: Growth colorless, changing to coral-pink. Aerial mycelium powdery, white.

Loeffler's coagulated serum: Same as on synthetic agar. No hydrolysis. Antagonistic properties: Produces an antiviral agent, abikoviromycin.

Remarks: No tyrosinase. It utilizes glycerol and glucose but not other carbohydrates.

110. Streptomyces viridis (Lombardo-Pelligrino emend. Krassilnikov). comb. nov. (Krassilnikov, N. A., Actinomycetales, Akad. Nauk. USSR, Moskau, 1941, 34.)

Vegetative growth: Green to dark green on all media. No soluble pigment.

Aerial mycelium: Well-developed on all media, cottony, whitish to grayish; sporophores long or short, straight, forming no spirals, but frequently producing broom-shaped clumps. Spores cylindrical, $1.0-1.5$ by $0.7-0.8 \mu$.

Gelatin: No liquefaction in 13 to 15 days.

Milk: No coagulation and no peptonization.

Starch: No hydrolysis.

Cellulose: No growth.

Nitrate: Reduction weak. 
Antagonistic properties: None; certain strains give postive activity.

Remarks: Not Streptomyces viridis of Baldacci, or of Millard and Burr (now Streptomyces viridogenes), or of Duché. According to Krassilnikov, Streptomyces lipmanii and Streptomyces verne represent strains of this species; Streptomyces viridis sterilis also listed as a strain that lost the capacity to produce aerial mycelium.

111. Streptomyces thermophilus (Gilbert) Waksman and Henrici. (Gilbert, Z. Hyg., 47, 1904, 383.)

Aerial mycelium: Hyphae straight, conidia formed.

Nutrient agar: No pigment.

Potato: Growth yellowish; no aerial mycelium. Plug usually colored brown.

Gelatin: Liquefaction. No pigment.

Milk: Proteolysis.

Starch: Growth yellowish; aerial mycelium white-gray. Hydrolysis good.

Czapek's agar: At $28^{\circ} \mathrm{C}$ growth deep, colorless; aerial mycelium thin, white; no soluble pigment.

Temperature: Optimum $50^{\circ} \mathrm{C}$. Good growth at $28^{\circ} \mathrm{C}$. Usually no growth at $60^{\circ} \mathrm{C}$. Some strains are incapable of growing at $28^{\circ} \mathrm{C}$, whereas others seem to grow well even at $65^{\circ} \mathrm{C}$.

Remarks: The culture Streptomyces thermophilus Berestnev is different. It produces spiral-shaped sporophores and spherical conidia. Growth is yellow-brown with brown soluble pigment. Aerial mycelium is velvety, dark green to gray-green. According to Noack's description, S. thermophilus produces a soluble bright red pigment.

112. Streptomyces thermodiastaticus (Bergey) comb. nov. (Bergey, D. H., J. Bacteriol. 4, 1919, 301.)

Aerial mycelium: Well-developed, white. Sporophores produce spirals; spores spherical to oval.

Synthetic agar: Growth colorless. Aerial mycelium well-developed, white.

Potato: Growth brownish. Aerial mycelium light gray.

Gelatin: Liquefaction slow.

Milk: No coagulation; no peptonization.

Starch: Hydrolysis strong.

Cellulose: Growth good.

Nitrate: Reduction.

Sucrose: Inversion.

Temperature: Optimum $65^{\circ} \mathrm{C}$. 
113. Streptomyces thermofuscus (Waksman, Umbreit, and Cordon) Waksman and Henrici. (Waksman, S. A., Umbreit, W. W., and Cordon, T. C., Soil Sci., 47, 1939, 49.)

Aerial mycelium: Hyphae spiral-shaped; conidia produced.

Potato: Growth abundant, dark-colored; no aerial mycelium, or few white patches, soluble pigment dark.

Gelatin: Liquefaction. At $50^{\circ} \mathrm{C}$, a grayish ring is produced and soluble pigment is formed. At $28^{\circ} \mathrm{C}$, growth with no soluble pigment.

Milk: Proteolysis.

Starch: Hydrolysis.

Czapek's agar: At $28^{\circ} \mathrm{C}$, growth poor, deep gray, with but little aerial mycelium. At $50^{\circ} \mathrm{C}$, growth dark to violet, with gray to lavender aerial mycelium and soluble brown pigment.

Temperature: Good growth at $50^{\circ}$ and $60^{\circ} \mathrm{C}$. Will grow at $65^{\circ} \mathrm{C}$. Faint growth at $28^{\circ} \mathrm{C}$.

Remarks: This species is distinguished from Streptomyces thermophilus by the brown-colored aerial mycelium on synthetic media, spiral-shaped hyphae, and ability to grow readily at $65^{\circ} \mathrm{C}$.

114. Streptomyces casei (Bernstein and Morton) comb. nov. (Bernstein, A., and Morton, H. E., J. Bacteriol., 27, 1934, 625.)

Vegetative growth: Colorless to white.

Aerial mycelium: White, $0.5-0.7 \mu$; no spirals.

Gelatin: Liquefaction complete.

Milk: Coagulation and peptonization.

Starch: No hydrolysis.

Nitrate: No reduction.

Temperature: Optimum $40^{\circ}-60^{\circ} \mathrm{C}$. Highly resistant to higher temperatures and to disinfectants. Thermal death point $100^{\circ} \mathrm{C}$.

Habitat: Pasteurized cheese.

Remarks: Krassilnikov places this culture in the same group with Actinomyces invulnerabilis of Acosta and Rossi, the latter being even more resistant to high temperatures and to disinfectants.

115. Streptomyces clavifer (Millard and Burr) comb. nov. (Millard, W. A., and Burr, S., Ann. Appl. Biol., 13, 1926, 580.)

Vegetative growth: Yellow to yellow-orange; soluble pigment yellowbrown.

Aerial mycelium: Sporophores long, straight, some terminating in clubshaped structures. Spores cylindrical, 1.5 by $1.0 \mu$. 
Synthetic sucrose agar: Gray to brick-red growth; white, sprinkled with cinnamon-drab, aerial mycelium. Yellowish to brown soluble pigment.

Synthetic glucose agar: Growth gray to brown; white to drab-colored aerial mycelium. Cinnamon-buff to brown soluble pigment.

Nutrient potato: Growth wrinkled gray to grayish olive; trace of aerial mycelium. Deep golden brown soluble pigment.

Potato: Growth wrinkled gray to orange to brown; gray to olive-buff aerial mycelium. Color of plug gray to brown.

Gelatin: Growth gray to buff; white aerial mycelium. Medium liquefaction. Yellow to reddish yellow soluble pigment.

Starch: Hydrolysis.

Glucose broth: Sponge-like growth at bottom of tube. Deep golden brown soluble pigment.

Tyrosinase reaction: Positive.

Nitrate: No reduction.

Glycerol synthetic solution: Growth in form of compact colonies at bottom and along sides of tube with some on surface, colored salmon to brown; scant drab aerial mycelium. Buff to golden soluble pigment.

Temperature: Fair growth at $37.5^{\circ} \mathrm{C}$.

Habitat: Limed soil and common scab of potatoes.

116. Streptomyces fimbriatus (Millard and Burr) comb. nov. (Millard, W. A., and Burr, S., Ann. Appl. Biol., 13, 1926, 580.)

Vegetative growth: Chocolate-colored.

Aerial mycelium: Mouse-gray. Sporophores form spirals with 3 or more turns. Spores cylindrical to oval, $1.2-0.9$ by $0.9 \mu$.

Synthetic sucrose agar: Growth gray; aerial mycelium abundant, white to gray, with a few specks of white. Soluble pigment cream-colored.

Synthetic glucose agar: Growth very good; aerial mycelium white to mouse-gray.

Nutrient potato agar: Colonies gray to blackish, flat, raised in center; a few specks of white aerial mycelium. Soluble pigment golden brown.

Potato: Growth mouse-gray; aerial mycelium on dried portions of growth scant white to mouse-gray. Pigment around growth black.

Gelatin: Growth good; aerial mycelium white. Liquefaction slow. Soluble pigment reddish.

Milk: Growth good. No coagulation and no hydrolysis.

Starch: Hydrolysis positive.

Glucose broth: Growth flocculated, mostly at bottom; no aerial mycelium. Soluble pigment golden brown.

Tyrosinase reaction: Strongly positive.

Nitrate: Reduction positive. 
Glycerol synthetic solution: Numerous colonies covering surface of medium and throughout medium; aerial mycelium scant white.

Habitat: Common scab of potatoes.

117. Streptomyces carnosus (Millard and Burr) comb. nov. (Millard, W. A., and Burr, S., Ann. Appl. Biol., 13, 1926, 580.)

Vegetative growth: Good, wrinkled, on synthetic and organic media.

Aerial mycelium: White to gray; spores cylindrical, 1.0 by $0.75 \mu$.

Synthetic sucrose agar: Growth pale smoke-gray covered with abundant, gray aerial mycelium. Colorless guttation drops appear over the whole surface.

Synthetic glucose agar: Growth pale olive-gray, covered with abundant white to gray aerial mycelium. Soluble pigment ivory-yellow to cartridgebuff.

Nutrient potato agar: Growth heavy, lichnoid, gray-colored. Aerial mycelium scant, white to gray; property lost on cultivation. Pigment light golden to brown.

Potato: Growth good, lichnoid, covered with gray to brownish aerial mycelium with white spots. The plug becomes colored gray to black.

Gelatin: Growth covered with aerial mycelium, white in center, gray in margin; liquefaction rapid.

Milk: Surface growth good. No aerial mycelium. Coagulation followed by liquefaction.

Starch: Hydrolysis.

Glucose broth: Whitish, sponge-like masses, sinking to bottom of tube.

Tyrosinase reaction: Negative.

Nitrate: Reduction.

Glycerol synthetic solution: Whitish to gray, discrete colonies, clinging to side or bottom of tube. Aerial mycelium scant, pale gray.

Habitat: Potato scab.

118. Streptomyces craterifer (Millard and Burr) comb. nov. (Millard, W. A., and Burr, S., Ann. Appl. Biol., 13, 1926, 580.)

Aerial mycelium: Much-branched; terminal branches dichotomously forked. Spores rectangular, $1.3-0.9$ by $1.0-0.8 \mu$.

Synthetic agar: Growth lichnoid, abundant, colorless; aerial mycelium mouse-gray. Numerous guttation drops, which leave blackish craters.

Nutrient agar: Growth colorless; aerial mycelium scant, white. No soluble pigment.

Potato: Growth good, cream-colored; aerial mycelium white to mousegray. Color of plug unchanged. 
Gelatin: Surface growth wrinkled; aerial mycelium white. Liquefaction rapid. No soluble pigment.

Milk: Surface growth cream-colored. No coagulation; peptonization rapid.

Starch: Growth spreading, thin, colorless; no aerial mycelium. Hydrolysis.

Glucose broth: Growth limited; aerial mycelium white to gray.

Tyrosinase reaction: Negative.

Nitrate: Reduction.

Glycerol synthetic solution: Good growth on surface of medium; numerous star-like colonies throughout medium; aerial mycelium scant, white to mouse-gray.

Temperature: Only slight growth at $37.5^{\circ} \mathrm{C}$.

Habitat: Raised, smooth scab.

119. Streptomyces gracilis (Millard and Burr) comb. nov. (Millard, W. A., and Burr, S., Ann. Appl. Biol., 13, 1926, 580.)

Vegetative growth: On synthetic media, dark brown, producing a soluble brown substance.

Aerial mycelium: Dark olive. Spiral-forming sporophores; spores oval or spherical, $0.8-0.9$ by $0.8 \mu$.

Synthetic sucrose agar: Growth fern-like, pale gray, covered with scant gray to buff aerial mycelium. Soluble pigment cream-colored.

Synthetic glucose agar: Growth smooth, pale olive-gray. Aerial mycelium abundant, white, smooth. Soluble pigment cream-colored.

Nutrient potato agar: Growth vinaceous buff to dark brown or almost black. Aerial mycelium gray. Soluble pigment light golden brown.

Potato: Growth good, echinate, covered with abundant olive-gray to buff aerial mycelium. Plug light brown.

Gelatin: Growth gray, covered with white aerial mycelium. Liquefaction rapid. Soluble pigment pink to dark golden brown.

Milk: Surface growth good; aerial mycelium white in the form of a ring and specks on surface. Coagulation slow, followed by rapid peptonization.

Starch: Hydrolysis.

Glucose broth: Growth good both on surface and on bottom. Aerial mycelium abundant, olive-buff. Soluble pigment light golden brown.

Nitrate: Reduction.

Glycerol synthetic solution: Growth good on surface and throughout medium. Aerial mycelium abundant, white to olive-buff.

Tyrosinase reaction: Negative.

Temperature: Grows well at $37.5^{\circ} \mathrm{C}$. 
Habitat: Potato scab.

Remarks: According to Krassilnikov, Streptomyces poolensis and Streptomyces tumuli belong to this group.

120. Streptomyces praecox (Millard and Burr) comb. nov. (Millard, W. A., and Burr, S., Ann. Appl. Biol., 13, 1926, 580.)

Vegetative growth: Raised, colorless.

Aerial mycelium: Well-developed, gray with greenish tinge. Sporophores produce open spirals. Spores spherical or oval, $0.8 \mu$ in diameter.

Synthetic agar: Growth thin, powdery; aerial mycelium white to olivebuff.

Synthetic glucose agar: Growth flat with radiating margins; aerial mycelium white to olive-colored. On cultivation, aerial mycelium is only white.

Nutrient agar: Growth thin, colorless. Aerial mycelium thin, white.

Potato: Growth good, cream-colored to light brown; aerial mycelium white to olive-buff. Plug olive-buff to drab; on cultivation, no soluble pigment produced.

Gelatin: Growth good; aerial mycelium abundant, white. Liquefaction medium.

Milk: Surface growth cream-colored, in form of ring. Aerial mycelium white. Coagulation marked; peptonization rapid.

Starch: Growth thin, cream-colored, aerial mycelium abundant, white, with greenish tinge. Hydrolysis.

Nutrient broth: Surface pellicle cream-colored. Aerial mycelium thin, white. No soluble pigment.

Glucose broth: Flocculated growth at bottom and heavy wrinkled growth at surface; aerial mycelium abundant, white.

Nitrate: Reduction variable.

Glycerol synthetic solution: Surface growth heavy; aerial mycelium abundant, powdery, white. Soluble pigment pale yellow.

Tyrosinase reaction: Negative.

Temperature: Grows well at $37.5^{\circ} \mathrm{C}$.

Odor: Very strong.

Antagonistic properties: Represses growth of Streptomyces scabies.

Habitat: Knob-like scab of potatoes.

Remarks: Krassilnikov considers this species related to Streptomyces griseus of Krainsky.

121. Streptomyces setonii (Millard and Burr) comb. nov. (Millar, W. A., and Burr, S., Ann. Appl. Biol., 13, 1926, 580.)

Vegetative growth: On synthetic media golden yellow. 
Aerial mycelium: Well-developed, cottony, dark gray to dark olive; sporophores straight, forming few spirals, spores oval-shaped, $0.6-0.8$ by $0.85 \mu$.

Synthetic agar: Growth abundant, smooth. Aerial mycelium, covering the whole surface, gray to olive-buff. Soluble pigment faint yellowish.

Nutrient agar: Growth good, colorless, covered with white, smooth aerial mycelium. Soluble pigment brownish.

Glucose agar: Growth good, lichnoid, gray to brown. Aerial mycelium abundant, white to olive-buff. Soluble pigment goldent brown.

Potato: Growth heavy, wrinkled, covered with abundant white to green to olive-buff aerial mycelium. Soluble pigment brownish to black.

Gelatin: Surface growth gray, covered with white aerial mycelium. Liquefaction rapid. Soluble pigment brownish.

Milk: Surface growth good, covered with ring of white aerial mycelium. Coagulation, followed by rapid digestion.

Starch: Growth cream-colored. Aerial mycelium patchy, white. Hydrolysis.

Glucose broth: Growth on surface and in liquid good. Aerial mycelium abundant, olive-buff. Soluble pigment light golden brown.

Nitrate: Reduction.

Glycerol synthetic solution: Growth flaky, white. Aerial mycelium abundant, olive-buff. Soluble pigment greenish.

Temperature: Grows well at $37.5^{\circ} \mathrm{C}$.

Habitat: Scabby potatoes.

Remarks: According to Krassilnikov, Streptomyces flavus, Streptomyces marginatus, Streptomyces praefecundus, Streptomyces tenuis, and Streptomyces loidensis represent merely strains of this species.

122. Streptomyces praefecundus (Millard and Burr) comb. nov. (Millard, W. A., and Burr, S., Ann. Appl. Biol., 1s, 1926, 580.)

Aerial mycelium: Spores oval-shaped, 0.8 by $0.85 \mu$.

Synthetic sucrose agar: Growth good, cream-colored, covered with abundant, cottony, olive-buff aerial mycelium. Soluble pigment cream-colored.

Synthetic glucose agar: Growth pale olive-gray, covered with abundant, smooth, olive-buff aerial mycelium. Soluble pigment light golden to buff.

Nutrient potato agar: Growth lichnoid, gray. Aerial mycelium smooth, white to yellowish. Soluble pigment golden brown.

Potato: Growth good, wrinkled, covered with abundant white to yellowish to olive-buff aerial mycelium Soluble pigment gray to brown.

Gelatin: Good surface growth, covered with white aerial mycelium. Light pink to dark golden brown soluble pigment. Liquefaction rapid. 
Milk: Surface growth good, covered with scant, white aerial mycelium. Coagulation, followed by peptonization.

Starch: Hydrolysis.

Glucose broth: Masses throughout medium flaky, whitish. Aerial mycelium abundant, pale olive-buff. Soluble pigment light golden brown.

Nitrate: Reduction.

Glycerol synthetic solution: Surface growth heavy, covered with white to olive-buff aerial mycelium. Soluble pigment none or very slight green.

Tyrosinase reaction: Negative.

Temperature: Grows well at $37.5^{\circ} \mathrm{C}$.

Habitat: Potato scab and soil.

123. Streptomyces viridogenes (Millard and Burr) comb. nov. (S. viridis of Millard, W. A., and Burr, S., Ann. Appl. Biol., 13, 1926, 580.)

Aerial mycelium: Filaments long, straight. Spores spherical $0.9 \mu$.

Synthetic sucrose agar: Growth abundant, covered with olive-grayish aerial mycelium. Soluble pigment greenish yellow to blackish green.

Synthetic glucose agar: Growth smooth, raised, olive-buff. Aerial mycelium abundant, light gray to deep mouse-gray. Soluble pigment yellowish to greenish yellow.

Nutrient agar with glucose: Growth gray to black. Aerial mycelium gray. Soluble pigment dark brown.

Nutrient agar: Growth lichnoid, cream-colored. No aerial mycelium. No soluble pigment.

Potato: Growth gray to olive-gray. Aerial mycelium either absent or white, turning gray. Soluble pigment brown.

Gelatin: Growth grayish, aerial mycelium scant, white to gray. Liquefaction rapid. Soluble pigment light golden brown.

Milk: Surface growth good; growth in medium cloudy. Aerial mycelium scant white. Coagulation rapid and peptonization gradual.

Starch: Growth gray to brown. Aerial mycelium thin, white. Hydrolysis.

Nutrient broth: Thin surface and bottom flaky growth, cream-colored. No soluble pigment.

Glucose broth: Growth on surface and at base good, flaky. Aerial mycelium gray to mouse-gray. Soluble pigment vinaceous to cinnamon-colored.

Nitrate: Reduction positive; occasionally negative.

Glycerol synthetic solution: Growth in medium and on surface flaky, white to vinaceous. Aerial mycelium gray.

Tyrosinase reaction: Negative.

Temperature: Grows well at $37.5^{\circ} \mathrm{C}$.

Habitat: Potato scab. 
124. Streptomyces loidensis (Millard and Burr) comb.nov. (Millard, W. A., and Burr, S., Ann. Appl. Biol., 18, 1926, 580.)

Aerial mycelium: Spores cylindrical to spherical, $0.9-1.0$ by $0.9-0.95 \mu$.

Synthetic sucrose agar: Growth thin, flat, gray to yellowish olive. Aerial mycelium scant, olive-colored. Soluble pigment yellow.

Synthetic glucose agar: Growth thin, grayish olive. Aerial mycelium olivebuff. Soluble pigment light golden to yellowish.

Nutrient potato agar: Growth good, gray; aerial mycelium olive-buff. Soluble pigment golden brown.

Gelatin: Growth gray, with scant white aerial mycelium. Liquefaction rapid. Soluble pigment yellow.

Milk: Surface growth excellent, covered with white aerial mycelium. Coagulation; peptonization rapid.

Starch: Hydrolysis.

Glucose broth: Growth on surface and at bottom, good, gray to golden brown. Aerial mycelium olive-buff. Soluble pigment golden brown.

Nitrate: No reduction.

Glycerol synthetic solution: Growth flaky; mostly at bottom. Aerial mycelium scant, buff-colored.

Tyrosinase reaction: Negative.

Temperature: Grows well at $37.5^{\circ} \mathrm{C}$.

Habitat: Potato scab.

125. Streptomyces wedmorensis (Millard and Burr) comb. nov. (Millard, W. A., and Burr, S., Ann. Appl. Biol., 13, 1926, 580.)

Aerial mycelium: Sporophores simple straight, branched, closely septated. Spores oval to cylindrical, $0.8-0.9$ by $0.6-0.8 \mu$.

Synthetic sucrose agar: Growth flat, thin, grayish, covered with white to gray aerial mycelium.

Synthetic glucose agar: Growth good, grayish, with crater-like dark spots. Aerial mycelium fair, gray.

Nutrient potato agar: Growth wrinkled, good, grayish. No aerial mycelium.

Potato: Growth wrinkled, grayish, covered with white aerial mycelium. Plug pigmented drab.

Gelatin: Growth fair. No aerial mycelium. Liquefaction medium.

Milk: Surface growth greenish. Coagulation; peptonization slow.

Starch: Hydrolysis.

Glucose broth: Small flakes and minute colonies at bottom and at surface.

Nitrate: Reduction.

Glycerol synthetic solution: Good growth in form of spongy masses at 
bottom and numerous colonies throughout medium; surface growth granular; aerial mycelium gray, flecked with white.

Tyrosinase reaction: Negative.

Temperature: Grows well at $37.5^{\circ} \mathrm{C}$.

126. Streptomyces scabies (Thaxter) Waksman and Henrici. (Thaxter, R., Ann. Rept. Conn. Agr. Exp. Sta., 1891, 153.)

Vegetative growth: Folded, compact, yellowish to yellow-brown; soluble pigment brown.

Aerial mycelium: White cottony or velvety; wavy or slightly curved hyphae show a few spirals. Spores cylindrical, $0.8-1.0$ by $1.2-1.5 \mu$.

Synthetic agar: Growth abundant, wrinkled, raised, gray to creamcolored. Aerial mycelium scarce, white to gray.

Nutrient agar: Colonies circular, entire, smooth, becoming raised, lichnoid, wrinkled, white to straw-colored, opalescent to opaque; no aerial mycelium; soluble pigment deep golden brown.

Glucose agar: Growth restricted, folded, cream-colored; aerial mycelium scant, white to gray.

Potato: Growth gray, opalescent, becoming wrinkled, black; aerial mycelium scant, grayish white. Color of plug brown.

Gelatin: Surface growth cream-colored, becoming brown. Liquefaction slow; soluble pigment yellowish.

Milk: Ring brown with greenish tinge. Coagulation; peptonization with alkaline reaction.

Starch: Hydrolysis.

Glucose broth: Ring in form of small colonies, settling to the bottom.

Nitrate: Reduction.

Tyrosinase reaction: Strong.

Temperature: Grows at $37^{\circ} \mathrm{C}$.

Antagonistic properties: Certain strains give positive effects, others negative.

Habitat: Numerous strains of this organism have been isolated from potato scab.

Remarks: Streptomyces clavifer, Streptomyces spiralis, Streptomyces carnosus, Streptomyces sampsonii, and many other species or strains are closely related to the type.

127. Streptomyces tenuis (Millard and Burr) comb. nov. (Millard, W. A., and Burr. S., Ann. Appl. Biol., 19, 1926, 580.)

Aerial mycelium: Irregularly branched. Spores cylindrical, 0.87 by $0.82 \mu$. Synthetic sucrose agar: Growth penetrates deep into substratum. Sur- 
face growth thin, flat, yellowish drab. Aerial mycelium deep olive-buff. Soluble pigment pale orange-yellow.

Synthetic glucose agar: Growth thin, flat, covered by olive-buff aerial mycelium. Soluble pigment green.

Nutrient potato agar: Growth wrinkled, grayish. Aerial mycelium white to vinaceous-fawn-colored. Soluble pigment golden brown.

Potato: Growth good, covered with deep olive-buff aerial mycelium. Soluble pigment gray to olive to black.

Gelatin: Growth pale gray, covered with scant white aerial mycelium. Liquefaction rapid. Soluble pigment yellow.

Milk: Growth good, covered with white aerial mycelium. Coagulation followed by incomplete peptonization.

Starch: Hydrolysis.

Glucose broth: Surface and bottom growth good, whitish. Aerial mycelium abundant, wrinkled, olive-buff. Soluble pigment golden brown.

Nitrate: No reduction.

Glycerol synthetic solution: Growth, mostly at bottom, white, flaky. Aerial mycelium scant, olive-buff.

Tyrosinase reaction: Negative.

Habitat: Potato scab.

128. Streptomyces marginatus (Millard and Burr) comb. nov. (Millard, W. A., and Burr. S., Ann. Appl. Biol., 13, 1926, 580.)

Aerial mycelium: Abundant, gray to yellow to olive-buff. Sporophores simple; spores spherical, 0.87 by $0.8 \mu$.

Synthetic sucrose agar: Growth thin, echinate, covered with abundant olive-buff aerial mycelium. Pigment cream-colored.

Synthetic glucose agar: Growth thin, yellowish, covered with white to buff aerial mycelium. Soluble pigment buff.

Nutrient potato agar: Growth heavy, gray, covered with white to whitish yellow aerial mycelium. Soluble pigment light golden brown to deep golden brown.

Potato: Growth good, raised, covered with abundant buff to olive-buff aerial mycelium. Plug at first gray, later becoming black.

Gelatin: Growth thin, pale olive-gray, covered with abundant pale gray to olive-buff aerial mycelium. Soluble pigment buff.

Milk: Growth good, flocculated; aerial mycelium white. Coagulation followed by peptonization.

Starch: Hydrolysis.

Glucose broth: White spongy mass at surface and on bottom, covered with white to yellow aerial mycelium. Soluble pigment light golden brown.

Nitrate: Reduction. 
Glycerol synthetic solution: Flaky growth at base and on surface. Aerial mycelium scant, olive-buff.

Tyrosinase reaction: Negative.

Temperature: Grows well at $37.5^{\circ} \mathrm{C}$.

Habitat: Potato scab.

129. Streptomyces salmonicolor (Millard and Burr) comb. nov. (Millard, W. A., and Burr, S., Ann. Appl. Biol., 13, 1926, 580.)

Vegetative growth: Hyphae minute.

Aerial mycelium: Poorly developed or absent entirely. Sporophores straight or form open spirals. Spores spherical or oval, $0.5-0.8$ by $0.4-0.8 \mu$.

Synthetic sucrose agar: Colonies minute, gray to pinkish. No aerial mycelium. No soluble pigment.

Synthetic glucose agar: Growth gray to purplish. No aerial mycelium. Soluble pigment faint golden.

Nutrient potato agar: Growth wrinkled, pinkish.

Potato: Growth restricted, wrinkled, raised, ocher-red to brown. Plug pigmented drab-gray.

Gelatin: Growth poor; liquefaction slow.

Milk: Surface growth fair. Coagulation; peptonization slow.

Starch: Hydrolysis.

Glucose broth: Growth in form of sponge-like mass.

Nitrate: Reduction.

Glycerol synthetic solution: Poor flaky growth at bottom of tube.

Tyrosinase reaction: Negative.

Temperature: Grows well at $37.5^{\circ} \mathrm{C}$.

130. Streptomyces maculatus (Millard and Burr) comb. nov. (Millard, W. A., and Burr, S., Ann. Appl. Biol., 13, 1926, 580.)

Vegetative growth: Dark green; hyphae extremely fine.

Aerial mycelium: Poorly developed, only on certain media as a fine gray cover. Sporophores short, straight. Spores spherical, 0.5-0.6 $\mu$. Chlamydospores produced.

Synthetic sucrose agar: Colonies round, flat, pale gray to pinkish, later showing dark green centers. No aerial mycelium.

Synthetic glucose agar: Growth thin, flat, almost black, with greenish tinge.

Nutrient potato agar: Growth vinaceous-tawny-colored, producing a vinaceous-tawny soluble pigment.

Potato: Growth restricted, raised, pinkish. Aerial mycelium scant, white. Soluble pigment gray to brown.

Gelatin: Growth poor; liquefaction slow. 
Milk: Growth very slight. No coagulation; no peptonization.

Starch: Hydrolysis.

Glucose broth: Numerous minute, whitish colonies at bottom of container.

Nitrate: No reduction.

Glycerol synthetic solution: Poor flaky growth at bottom.

Tyrosinase reaction: Negative.

Temperature: Grows well at $37.5^{\circ} \mathrm{C}$.

Anerobic growth: Good.

131. Streptomyces coroniformis (Millard and Burr) comb. nov. (Millard, W. A., and Burr, S., Ann. Appl. Biol., 13, 1926, 580.)

Aerial mycelium: Simple branching. Spores oval, 0.8 by $0.6 \mu$.

Synthetic sucrose agar: Growth in form of discrete colonies partially coalescing, gray to greenish. White aerial mycelium covering edges of growth.

Synthetic glucose agar: Growth in form of discrete colonies growing into the medium, poor grayish. No aerial mycelium.

Nutrient potato agar: Growth wrinkled, grayish. No aerial mycelium.

Potato: Growth raised, grayish. Aerial mycelium white. Plug pigmented brownish around and under growth.

Gelatin: Growth fair. Liquefaction slow or none.

Milk: A few colonies on surface. No coagulation. Peptonization limited.

Starch: Hydrolysis negative to trace.

Glucose broth: Fair growth at bottom.

Nitrate: Reduction limited.

Glycerol synthetic solution: Very poor growth in form of small flakes at bottom.

Tyrosinase reaction: Negative.

Temperature: Growth fair at $37.5^{\circ} \mathrm{C}$.

Habitat: Potato scab.

132. Streptomyces spiralis (Millard and Burr) comb. nov. (Millard, W. A., and Burr, S., Ann. Appl. Biol., 13, 1926, 580.)

Vegetative growth: Lichnoid, smooth, yellowish golden. No soluble pigment.

Aerial mycelium: Sporophores straight or spiral-forming. Spores cylindrical, $1.0-1.7$ by $0.9 \mu$.

Synthetic sucrose agar: Growth rough or granular; aerial mycelium vinaceous-buff to dark grayish olive. Soluble pigment pale vinaceous-fawncolored. 
Synthetic glucose agar: Growth fairly rough, pale gray; aerial mycelium scant, white. Soluble pigment yellowish to pink.

Nutrient potato agar: Growth granular, gray; aerial mycelium abundant, white to olive-buff. Soluble pigment light golden brown.

Potato: Growth poor, wrinkled, grayish vinaceous; aerial mycelium white to grayish vinaceous. Plug colored brown around and below growth.

Gelatin: Growth limited, gray; aerial mycelium scant, white. Liquefaction rapid.

Milk: Surface growth good, covered with abundant white aerial mycelium. Coagulation; peptonization rapid.

Starch: No hydrolysis.

Glucose broth: Minute compact colonies at bottom and on surface. Aerial mycelium scant, white.

Nitrate: No reduction.

Glycerol synthetic solution: No growth.

Tyrosinase reaction: Negative.

Temperature: Does not grow at $37.5^{\circ} \mathrm{C}$.

Habitat: Grass compost.

133. Streptomyces sampsonii (Millard and Burr) comb. nov. (Millard, W. A., and Burr, S., Ann. Appl. Biol., 1s, 1926, 580.)

Aerial mycelium: Long branching aerial hyphae. Spores cylindrical, 0.81.0 by $0.5 \mu$ (spores oval to spherical, $\mathrm{WG}^{*}$ ).

Synthetic sucrose agar: Growth good, wrinkled, pale gray to white. Aerial mycelium very scant, white. Soluble pigment green to buff.

Synthetic glucose agar: Growth good, wrinkled, white to gray. Aerial mycelium scant, white. Soluble pigment yellow to brownish.

Potato: Growth wrinkled, grayish. Aerial mycelium white. Soluble pigment golden brown (none, WG).

Gelatin: Surface growth scant, gray. Aerial mycelium trace of whitish. Liquefaction rapid.

Milk: Surface growth good, whitish. No aerial mycelium. No coagulation; no peptonization (peptonization rapid, WG.)

Starch: No hydrolysis (rapid hydrolysis, WG).

Glucose broth: Good surface and bottom growth, as well as many colonies clinging to side of tube. Aerial mycelium white.

Nitrate: Reduction.

Glycerol synthetic solution: No growth.

Tyrosinase reaction: Negative.

Temperature: $28^{\circ} \mathrm{C}$.

Habitat: Potato scab.

* WG $=$ Observation made in our laboratory by Waksman and Gordon. 
134. Streptomyces intermedius (Krüger emend. Wollenweber) comb. nov. (Wollenweber, H., Ber. Deut. Botan. Ges., 39, 1922, 26.)

Vegetative growth: Original description recorded colonies green-colored, soluble pigment green. On continued cultivation, green color tends to become cream-colored to brownish.

Aerial mycelium: Light gray to gray. Sporophores straight, frequently arranged in clumps. Spores elongated, $0.9-1.0$ by $0.7 \mu$.

Synthetic agar: Growth slightly folded, cream-colored to brown. Aerial mycelium thin, white.

Nutrient agar: Growth much-folded, cream-colored. Aerial mycelium in upper portions of slant white. Soluble pigment faintly golden.

Glucose agar: Growth good, brownish. Aerial mycelium heavy, creamcolored. No soluble pigment.

Potato: Growth folded, brown. Trace of white aerial mycelium in upper, drier portions of growth. No soluble pigment.

Gelatin: Growth thin, colorless to faintly brown, dropping to bottom. No soluble pigment. Liquefaction slow.

Milk: Surface growth heavy, cream-colored. No aerial mycelium. No coagulation; peptonization slow.

Starch: Growth same as on synthetic agar. Hydrolysis good.

Nutrient broth: Film thin, colorless, dropping to bottom. No aerial mycelium. No soluble pigment.

Nitrate: Reduction limited.

Sucrose: Inversion slow.

Antagonistic properties: Positive.

Habitat: Potato scab.

135. Streptomyces ipomoea (Person and Martin) Waksman and Henrici. (Person, L. H., and Martin, W. J., Phytopathology, 30, 1940, 913.)

Conidia on glucose-casein agar: Oval to elliptical, $0.9-1.3$ by $1.3-1.8 \mu$.

Synthetic agar: Growth abundant, mostly on surface of medium, moderately wrinkled, olive-yellow.

Nutrient agar: Moderate growth in the form of small, shiny, crinkled colonies both on the surface and imbedded in the medium, silver-colored.

Potato: Growth moderate, shiny, wrinkled, light brown. No aerial mycelium. No soluble pigment.

Gelatin: After 25 days at $20^{\circ} \mathrm{C}$, scanty growth, no aerial mycelium; no soluble pigment; liquefaction.

Milk: Growth in form of ring; hydrolysis, without visible coagulation.

Starch: Growth moderate, smooth, deep in medium, ivory-colored. Aerial mycelium white with patches of bluish green. No soluble pigment. Complete hydrolysis after 12 days. 
Cellulose: No growth.

Nitrate: Reduction to nitrite.

Antagonistic properties: Positive.

Habitat: Sweet potato disease.

136. Streptomyces poolensis (Taubenhaus) Waksman and Henrici. (Taubenhaus, J. J., J. Agr. Research, 13, 1918, 446.)

Aerial mycelium: Fine, branching; spirals usually not seen. Conidia oval to elliptical.

Synthetic agar: Growth thin, spreading, colorless. Aerial mycelium white to gray.

Nutrient agar: Growth translucent, yellowish.

Glucose agar: Growth abundant, glossy, raised center, entire, light brown.

Potato: Growth thin, reddish brown; medium becoming purplish.

Gelatin: Liquefaction, with small, brownish flakes in fluid.

Milk: Ring brownish. Coagulation; peptonization, with strongly alkaline reaction.

Starch: Growth restricted, cream-colored. No hydrolysis.

Glucose broth: Ring thin, brownish.

Nitrate: Reduction to nitrite.

Temperature: $37^{\circ} \mathrm{C}$.

Antagonistic properties: Positive.

Habitat: Sweet potato "pox."

Remarks: Faint trace of soluble brown pigment.

137. Streptomyces tumuli (Millard and Beeley) comb. nov. (Millard, W. A., and Beeley, F., Ann. Appl. Biol., 14, 1927, 296-311.)

Synthetic agar: Growth, penetrating into the medium, gray, later becoming darkly opaque. Aerial myceliun arises on center of growth, at first white, later becoming pale gray. Surface of growth covered with colorless drops leaving small black craters. No soluble pigment.

Nutrient agar: Growth good, lustrous, slimy, gray. No aerial mycelium. No soluble pigment.

Glucose agar: Growth wrinkled, pale gray. White aerial mycelium arising in concentric rings around a dark bare center. Soluble pigment olive-colored.

Potato: Growth heavy, slimy, black. No aerial mycelium. Plug becoming grayish brown.

Gelatin: Growth beaded. No aerial mycelium. Liquefaction rapid. No soluble pigment.

Milk: Growth good; no aerial mycelium. Coagulation; peptonization slight.

Starch: Hydrolysis. 
Nutrient broth: Colonies large, spherical, white. No soluble pigment. Nitrate: Reduction.

Temperature: Grows well at $37^{\circ} \mathrm{C}$.

Habitat: Isolated from mound scab of mangels.

138. Streptomyces listeri (Erikson) Waksman and Henrici. (Erikson, D., Med. Research Council Spec. Rept. Ser. 203, 1935, 36.)

Vegetative growth: Long slender filaments, many loosely wavy, forming a dense spreading mycelium which rapidly grows into a membrane on most media.

Aerial mycelium: Very slow and inconstant in appearance, short and straight, conidia oval.

Nutrient agar: Growth smooth, round, moist, cream-colored, margin depressed, center elevated, closely adherent; becoming umbilicated, with a myceloid margin.

Glucose agar: Cream-colored, glistening, pinpoint colonies; later aggregated in convoluted skin.

Potato: Abundant, dull, brownish, wrinkled skin with white aerial mycelium; large, stellate, fluffy, white colonies in liquid at base.

Gelatin: Liquefaction slight; round white surface colonies; after 45 days, confluent skin, almost completely liquefied.

Milk: Coagulation. No change in reaction.

Glycerol agar: Growth abundant, moist, cream-colored, colonies elevated, piled up; aerial mycelium powdery, white. After 20 days, skin deeply buckled; colorless with exuded drops.

Calcium agar: Growth poor, a slight biscuit-colored membrane.

Potato agar: After 1 week, extensive growth, colorless submerged colonies, warted surface; dirty pink coloration after 2 weeks; scant white aerial mycelium after 4 months.

Dorset's egg medium: No growth.

Blood agar: Small, round, cream-colored colonies, smooth translucent surface; no hemolysis.

Serum agar: Small, irregular, moist, cream-colored colonies, tending to be heaped up; later somewhat transparent.

Glucose broth: Small, white, nodular colonies; later abundant flocculi.

Nutrient broth: Small, round, white colonies in sediment.

Source: From human material. Strain from Lister Collection.

139. Streptomyces galtieri (Goret and Joubert) comb. nov. (Goret, P., and Joubert, L., Actinomycetales, Ann. Parasitol. Humaine et Comparée, 26, 1951, 118-127.) 
Vegetative growth: Wavy mycelium branched in a monopodial form, $1 \mu$ in diameter. On agar, two types of colonies are produced-one small, flat, regular, white; the other large, thick, irregular, yellowish.

Aerial mycelium: Producing spirals; conidia oval, $0.8-1.5$ by $0.8 \mu$.

Synthetic agar: Colonies small; aerial mycelium powdery, white. No soluble pigment.

Nutrient agar: Growth poor, thin, yellowish; aerial mycelium powdery, white. Soluble pigment brown.

Peptone agar: Growth limited, cream-colored; aerial mycelium powdery, white. Soluble pigment very slight, brown-reddish.

Gelatin: Growth poor, flaky, white; liquefaction limited.

Milk: Growth slow; aerial mycelium white. No soluble pigment. At $25^{\circ} \mathrm{C}$ no coagulation; at $37^{\circ} \mathrm{C}$ coagulation after 20 days. No peptonization. No change in reaction.

Starch: Colonies thin; aerial mycelium powdery, white. No soluble pigment.

Nitrate: Reduction.

Glucose-peptone medium: Numerous colonies covering surface. Soluble pigment slight, brownish.

Glycerol potato: Orange-reddish punctiform colonies growing together. A thick crust. Limited white aerial mycelium appearing very slowly. No soluble pigment.

Proteolysis: No action on coagulated serum.

Source: Dog septicemia (thoracic, abdominal, and brain lesions).

Remarks: Pathogenic for guinea pig and rabbit. Not pathogenic for dog after laboratory growth.

140. Streptomyces upcottii (Erikson) Waksman and Henrici. (Gibson, A. G., J. Pathol., and Bacteriol., 23, 1920, 357; Erikson, D., Med. Research Council Spec. Rept. Ser. 203, 1935, 36.)

Vegetative growth: Filaments characteristically long, straight, much interwoven and ramified; typical unicellular mycelium, usually forming medium to large heavy cartilaginous colonies.

Aerial mycelium: A very slight transient aerial mycelium appeared on one agar slope, but this has not been repeated on any slide microculture on any medium.

Nutrient agar: Smooth, shining, round, cream-colored colonies, margin submerged, scant white aerial mycelium in 1 week; colonies large (up to $10 \mathrm{~mm}$ in diameter), centers elevated, greenish tinge, very sparse aerial mycelium in 3 weeks; the aerial mycelium disappears and large radial grooves appear in most colonies in 3 weeks. 
Glucose agar: Smooth, round, cream-colored colonies, margin depressed, centers elevated, hollow on reverse side; later a coherent membranous growth, piled up, yellowish.

Gelatin: Abundant flocculent growth along streak, round cream-colored colonies on surface. Partial liquefaction in 14 days; complete liquefaction in 2 months.

Glycerol agar: Small, round, cream-colored, glistening colonies, heavy texture, margins submerged; later, colonies umbilicated, tending to be piled up; after 6 weeks, growth very much convoluted and raised, broad submerged margin, slightly reddish medium.

Calcium agar: Small colorless membranous growth with undulating margin; later, centrally depressed into medium.

Potato agar: Growth poor, small, colorless blister colonies, medium slightly discolored.

Dorset's egg medium: Round, flat, colorless, scale-like colonies, some marked by concentric rings and slightly hollowed in center; growth becomes yellow-brown.

Blood agar: Large, drab, heavily textured colonies; no aerial mycelium; no hemolysis.

Serum agar: Large colonies (3 to $4 \mathrm{~mm}$ in diameter), colorless, granular, centrally elevated, depressed at margin, resembling limpets.

Nutrient broth: Large coherent mass composed of fluffy colonies.

Carrot plug: Colorless, spreading, moist, wrinkled growth in 6 weeks; later a dull greenish brown, moist, very much wrinkled and depressed skin.

Source: From the spleen in a case of acholuric jaundice.

Remarks: Slightly acid-fast.

141. Streptomyces hortonensis (Erikson) Waksman and Henrici. (Erikson, D., Med. Research Council Spec. Rept. Ser. 203, 1935, 36.)

Vegetative growth: Typical germination into very slowly growing unicellular mycelium composed of long slender straight branching filaments.

Aerial mycelium: Very sparse, straight aerial mycelium produced only once on potato. Non-acid-fast.

Nutrient agar: Very slow growth, a few smooth, cream-colored, coiled colonies in 19 days; after 2 months, liberal, irregular convoluted growth.

Glucose agar: Coiled and heaped up cream-colored translucent masses; after 2 months, growth rounded, elevated, ridged outward from hollow center.

Potato: After 3 weeks, abundant, colorless, umbilicated, round colonies, some coiled in raised masses; later, liberal olive-green growth, piled up, dense, velvety gray-green aerial mycelium at top of slant, small, round, fluffy white colonies in liquid at base. 
Gelatin: Round cream-colored colonies on surface and a few mm below. No liquefaction.

Milk: Surface growth green, liquid hydrolyzed, partly clear, purple; later decolorized, brown.

Glycerol agar: Coiled, colorless, lustrous patches, isolated colony with central depression.

Potato agar: Colorless blister colonies in 1 week; dull green heaped and coiled mass after 3 weeks; medium becomes slightly discolored.

Serum agar: Poor growth; small, amorphous, cream-colored mass.

Source: From pus containing typical actinomycotic granules from parotid abscess.

142. Streptomyces beddardii (Erikson) Waksman and Henrici. (Erikson, D., Med. Research Council Spec. Rept. Ser. 203, 1935, 36.)

Vegetative growth: Rapidly growing, dense, spreading mycelium composed of very long slender filaments, many wavy or closely coiled, particularly on glucose agar; spirals less marked or lacking on poorer nutritive media like synthetic glycerol agar or water agar.

Aerial mycelium: Sparse, short, straight on synthetic glycerol agar, much slower and more plentiful on glucose agar; later shows long, very fine spirals breaking up into small oval conidia; aerial hyphae straighter and more branched with shorter conidiophores on starch agar.

Nutrient agar: Colorless, coherent, wrinkled, membranous growth with submerged margin; after 3 months, medium discolored, scant white aerial mycelium.

Glucose agar: Wrinkled membranous growth; after 2 months, scant white aerial mycelium.

Potato: Growth moist, membranous, colorless, with scant white aerial mycelium at top of plug.

Gelatin: Dull white flakes sinking to bottom as medium liquefies; liquefaction complete in 8 days.

Milk: Coagulation followed by peptonization.

Starch: Growth spreading, colorless; considerable white aerial mycelium. Hydrolysis.

Nutrient broth: Suspended and sedimented colorless flocculi, some small round colonies.

Glycerol agar: Small, cream-colored, discrete colonies becoming confluent, under surface much buckled.

Calcium agar: Growth extensive, moist, wrinkled, membranous, creamcolored.

Potato agar: Moist, cream-colored skin, convoluted, closely adherent. 
Dorset's egg medium: Growth extensive, very wrinkled, membranous, surface bright yellow. After 2 months, considerable liquefaction.

Blood agar: Hemolysis. Growth in uniformly striated colorless bands, occasional round colonies at margin.

Serum agar: Growth wrinkled, glistening, membranous, cream-colored.

Inspissated serum: Growth smeary, colorless, reverse becoming transparent, starting to liquefy at base; completely liquefied and brown in 12 days.

Synthetic glycerol solution: At first, a few round white colonies in suspension; later, large, branched, feathery mass at bottom.

Source: Human spleen in a case of splenic anemia.

143. Streptomyces kimberi (Erikson) Waksman and Henrici. (Erikson, D., Med. Research Council Spec. Rept. Ser. 203, 1935, 36.)

Vegetative growth: Mycelium of long, straight, profusely branching filaments forming circumscribed colonies on all media.

Aerial mycelium: Abundant, short, straight, and branched; small round conidia.

Nutrient agar: Colonies smooth, round, moist, cream-colored, $1 \mathrm{~mm}$ in diameter; after 17 days, aerial mycelium powdery white.

Glucose agar: Discrete cream-colored colonies becoming confluent; aerial mycelium white.

Gelatin: Liquefaction. Smooth shining colonies becoming powdery white with aerial mycelium, floating on liquefied medium. No pigmentation.

Milk: Coagulation; no peptonization; initial pinkish brown ring descends until medium is dark brown throughout ( 2 months).

Starch: No hydrolysis.

Nutrient broth: Small, round colonies in sediment in 2 days; supernatant colonies with white aerial mycelium and large hollow flakes in sediment in 15 days; occasional reddish brown coloration.

Glycerol agar: Moist cream-colored colonies becoming confluent; aerial mycelium white.

Calcium agar: Dull, cream-colored, scaly growth, covered by chalky, white aerial mycelium.

Potato agar: Extensive growth covered by white, powdery aerial mycelium; large, colorless exuded droplets.

Wort agar: Heavy, brownish, lichnoid colony; after 30 days, white aerial mycelium.

Czapek's agar: Small colonies covered with white aerial mycelium.

Dorset's egg medium: Closely adherent scale-like colonies, centrally elevated, with white aerial mycelium. 
Serum agar: Moist, cream-colored honeycombed skin; aerial mycelium scant, white.

Synthetic glycerol solution: Round white colonies at bottom; later coherent mulburry-like mass composed of fluffy round portions; after 15 days, irregular wispy flocculi and large coherent mass.

Source: Blood culture of a woman with acholuric jaundice.

144. Streptomyces somaliensis (Brumpt) Waksman and Henrici. (Brumpt, Arch. Parasitol., Paris, 10, 1906, 489; Précis de Parasitologie, Paris, 2nd ed., 1913, 967.)

Vegetative growth: Simple branching unicellular mycelium with long straight filaments, forming circumscribed colony.

Aerial mycelium: Short, straight.

Nutrient agar: Growth abundant, granular, yellowish, with small discrete colonies at margin; later growth colorless, colonies umbilicated.

Glucose agar: Growth poor, elevated patch, moist, cream-colored.

Potato: Abundant growth, colonies round and oval, partly piled up in rosettes, frosted with whitish gray aerial mycelium, plug discolored; after 16 days, aerial mycelium transient, growth nearly black.

Gelatin: Cream-colored colonies, medium pitted; complete liquefaction in 10 days; hard black mass at bottom.

Milk: Soft semiliquid coagulum which undergoes digestion; heavy wrinkled surface pellicle, completely liquefied in 12 days.

Nutrient broth: A few round white colonies at surface, numerous fluffy masses in sediment; later large irregular mass breaking into wisps.

Glycerol agar: Growth abundant, minute round to large convoluted and piled up masses, colorless to dark gray and black.

Calcium agar: Round cream-colored colonies, depressed, umbilicated, piled up; aerial mycelium thin, white; colonies become pale brown.

Doreset's egg medium: Extensive colorless growth, partly discrete; becoming opaque, cream-colored, very wrinkled; later rough, yellow, mealy, portion liquid.

Blood agar: Small dark brown colonies. round and umbilicated, piled up in confluent bands, reverse red-black; hemolysis.

Serum agar: Spreading yellow-brown skin, intricately convoluted.

Antagonistic properties: Positive.

Source: Yellow-grained mycetoma, Khartoum.

Remarks: Although Streptomyces somaliensis has been known for a long time, there have been, until recently, no detailed descriptions of the organism beyond the fact that it possesses a distinctly hard sheath around the grain which is insoluble in potash and eau de javelle. The rare occur- 
rence of septa and occasional intercalary chlamydospores is reported by Brumpt (Arch. Parasitol., 10, 1905, 562), but has not been confirmed by Erikson (loc. cit.). Chalmers and Christopherson (Ann. Trop. Med. Parasitol., 10, 1916, 223) merely mentioned the growth on potato as yellowish white and lichnoid without describing any aerial mycelium. Balfour in 1911 reported a case but gave no data, and Fülleborn limited his description to the grain (Arch. Schiffs, Trop. Hyg., 15, 1911, 131). This species was first placed in Indiella, a genus of fungi, by Brumpt (1906, loc. cit.). Later Brumpt (1913, loc. cit.) proposed a new genus or subgenus, Indiellopsis, containing the single species Indiellopsis somaliensis.

145. Streptomyces panjae (Erikson) Waksman and Henrici. (Erikson, D., Med. Research Council Spec. Rept. Ser. 203, 1935, 36.)

Vegetative growth: Unicellular mycelium with slender, branching filaments; very small, round colonies.

Aerial mycelium: No aerial mycelium visible on any medium, but occasional isolated aerial branches.

Nutrient agar: Growth irregularly piled up, convoluted, colorless; after 1 month, easily detachable, brownish.

Glucose agar: Small colorless coiled mass in 1 week; heaped up green growth in 2 weeks.

Gelatin: Liquefaction complete in 4 days.

Milk: Coagulation; pale green surface growth; mostly digested in 2 weeks.

Glucose broth: Growth poor, flakes, scant, pinkish.

Nutrient broth: Flakes and minute colorless colonies.

Glycerol agar: Poor growth, scant colorless patch.

Calcium agar: Colorless to pink spreading growth with minute, discrete colonies at margin; after 2 weeks, bright red mass, buckled and shining, colorless submerged margin.

Potato agar: Small, elevated, convoluted, colorless masses with purple tinge in center.

Dorset's egg medium: Small, round, tough, colorless colonies, margin well embedded; after 3 weeks, colonies elevated, warted, darkened, medium discolored and broken; slight degree of liquefaction, medium dark brown.

Source: An ulcer of the abdominal wall, Calcutta.

146. Streptomyces willmorei (Erikson) Waksman and Henrici. (Erikson, D., Med. Research Council Spec. Rept. Ser. 203, 1935, 36.)

Vegetative growth: Unicellular mycelium frequently branches at short intervals, presenting peculiar clubbed and budding forms with occasional separate, round, swollen cells. The filaments are characteristically long, homogeneous, and much-interwoven. 
Aerial mycelium: Profuse in most media, with a marked tendency to produce loose spirals with chains of ellipsoidal conidia. Thick aerial clusters may also be formed.

Nutrient agar: Growth heavy, colorless, lichnoid, rounded elevations covered with white aerial mycelium; later, submerged margin, round confluent growth, aerial mycelium marked in concentric zones.

Glucose agar: Growth colorless, wrinkled, confluent, with smooth entire margin; large discrete colonies like flat rosettes; after 4 months, aerial mycelium scant, white.

Gelatin: Colonies minute, colorless; liquefaction.

Milk: Coagulation; one-third peptonized.

Nutrient broth: Large, fluffy, white hemispherical colonies, loosely coherent.

Glycerol agar: Round, smooth, cream-colored colonies, heavy texture, margin submerged; stiff sparse aerial spikes; after 3 weeks, colonies large (up to $10 \mathrm{~mm}$ in diameter).

Calcium agar: Spreading colorless growth, pitting medium, submerged undulating margin; aerial mycelium very scant, white.

Potato agar: Fair growth, partly submerged, covered with grayish white aerial mycelium; medium becomes discolored.

Dorset's egg medium: Large, round, colorless, scale-like colonies, radially wrinkled; growth brownish, medium discolored in 2 weeks.

Serum agar: Smooth colorless discoid colonies; marked umbilication after 2 weeks.

Carrot plug: Colorless raised colonies with powdery white aerial mycelium; after 1 month, very much piled up, aerial mycelium gray; after 2 months, superabundant growth around back of plug, confluent, greatly buckled, all-over gray aerial mycelium.

Antagonistic properties: Positive.

Source: Streptothricosis of liver.

147. Streptomyces sterilis (Krassilnikov) comb. nov. (Krassilnikov, N. A., Actinomycetales, Akad. Nauk. USSR, Moskau, 1941, 24, 35, 52).

In view of the fact that various species of Streptomyces are able to lose the capacity to produce aerial mycelium, either on continued cultivation or by a sort of mutation, cultures are obtained which may be mistaken for nocardias. They can be recognized, however, by the structure of their vegetative mycelium and by their cultural and physiological properties, such as formation of soluble pigments, liquefaction of gelatin, hydrolysis of starch, inversion of sucrose, coagulation and peptonization of milk. Occasionally some are able to revert to the typical streptomycetes or regain the capacity to produce aerial mycelium. 
Such cultures represent many species. Their growth is more commonly colorless, but sometimes pigmented, smooth or lichnoid, leathery, compact, with shiny surface. Some produce a soluble brown pigment. This was recognized by Krassilnikov, who designated such cultures as Actinomyces albus sterilis and A. viridis sterilis, similar to the formation of Fungus sterilis. $\mathrm{He}$ isolated from the soil about 100 such cultures. These were divided into three groups:

1. Strongly proteolytic cultures, capable of liquefying gelatin in 3-5 days, of peptonizing milk in 6-10 days, with or without preliminary coagulation, of hydrolyzing starch with varying degrees of rapidity, of inverting sugar No growth on cellulose. Strongly antagonistic.

2. Gelatin slowly liquefied, in 15-30 days, or not at all in that time; milk coagulated and peptonized simultaneously; starch hydrolyzed with varying degrees of rapidity or not at all. No growth on cellulose. Weak antagonistic properties.

3. Milk coagulated, due to acidification, but not peptonized. No antagonistic effects.

In our collection, many of the cultures that originally produced aerial mycelium have lost the capacity to do so, and can, therefore, no longer be considered as type cultures. Streptomyces griseus, a vigorously growing culture capable of producing streptomycin, yielded a mutant, which no longer produces aerial mycelium, nor is it able to produce streptomycin.

On the other hand, certain nocardia-like organisms have been isolated from natural substrates, which, on continued cultivation on artificial media, gave rise to variants which produced sporulating aerial mycelium. This is true, for example, of the culture designated by Gause as Proactinomyces cyaneus-antibioticus and thought to be identical with Beijerinck's Actinococcus cyaneus. Beijerinck believed in a close relationship between his cultures and that of Streptomyces coelicolor Müller. Whether these cultures are naturally occurring sterile forms of Streptomyces, whether they are natural mutants, or whether, as Gause belived, Streptomyces can be a mutant of Nocardia (Proactinomyces), remains to be determined. 


\section{Description of Species of Micromonospora}

1. Micromonospora chalcea (Foulerton) Orskov. (Foulerton, A., Lancet, 1, 1905, 1200; Orskov, J., Investigations into the morphology of the ray fungi, Copenhagen, 1923.)

Vegetative growth: Rapid growth on all nutrient media, especially on glucose-asparagine agar. Growth heavy, compact, raised, pale pink to deep orange, not spreading much into the medium. Hyphae long, thin, branching, nonseptate. Surface of growth smooth or folded, dull or shining. No soluble pigment.

Aerial mycelium: None. Spore-layer well developed, moist and glistening, brownish black to greenish black; color sometimes spreading through the whole mass of growth. Conidia oval or spherical, formed individually on relatively nonbranching conidiophores.

Gelatin: Liquefaction positive.

Milk: Peptonization, occasional coagulation.

Starch: Hydrolysis positive.

Cellulose: Rapid decomposition.

Nitrate: Reduction to nitrite.

Sucrose: Inversion positive.

Proteolytic action seems stronger in this than in the other species of this genus.

Temperature: Optimum for growth $30^{\circ}-35^{\circ} \mathrm{C}$. Thermal death point of mycelium, $70^{\circ} \mathrm{C}$ in 2 to 5 minutes. Spores resist $80^{\circ} \mathrm{C}$ for 1 to 5 minutes.

Source: Soil, lake mud, and other substrates.

2. Micromonospora fusca Jensen. (Jensen, H., Proc. Linnean Soc. N. S. Wales, 57, 1932, 178.)

Vegetative growth : On glucose-asparagine agar heavy, compact, orangecolored, rapidly changing to deep brown and nearly black. Deep brown soluble pigment.

Aerial growth: Spore-layer moist, glistening, grayish to brownish black.

Gelatin: Liquefaction weak. Grows in liquid media as small brown granules and flakes. Soluble pigment very slight.

Milk: Slow digestion; no coagulation; slight grayish brown discoloration.

Starch: Hydrolysis positive.

Cellulose: Limited attack.

Nitrate: Reduction positive or negative. 
Sucrose: Inversion positive.

Antagonistic properties: Produces micromonosporin.

Source: Soil.

3. Micromonospora parva Jensen. (Jensen, H., Proc. Linnean Soc. N. S. Wales, 57, 1932, 177.)

Vegetative growth: Scant growth on glucose-asparagine agar; mycelium thin, spreading widely into the agar, almost colorless to pale pink or orange.

Aerial growth: Sporulation scant, giving rise to thin grayish, moist crusts on the surface; spores oval, in mass gray-colored.

Gelatin: Liquefaction positive.

Milk: Unchanged; or coagulated, slowly redissolved with faintly acid reaction.

Starch: Hydrolysis positive.

Cellulose: No decomposition.

Nitrate: No reduction.

Sucrose: No inversion.

Source: Soil.

4. Micromonospora globosa Krassilnikov. (Krassilnikov, N. A., Ray fungi and related organisms, Izv. Akad. Nauk, Moskau, 1938, 134; Microbiology (USSR), 8, 1939, 179.)

Vegetative growth: Rugose, at first very compact, later acquires a pasty consistency, and bond with the medium becomes less fast. Color of cultures varies from light yellow to orange-red. During fruit-bearing, colonies are covered with a brownish black tarnish of conidia.

Aerial growth: Conidia are formed at the ends of short branches, one on each. Individual branches with conidia resemble grapevines. Conidia spherical, 1.0-1.3 $\mu$; they arise by the swelling of the branch tips. The swellings become round, acquire the shape of spheres, which, as the formation of the conidia proceeds, are divided from the branch by a transverse septum.

Milk: Coagulation; peptonization.

Starch: Hydrolysis.

Cellulose: No decomposition.

Nitrate: Reduction to nitrite.

Sucrose: Inversion.

Source: Soil.

5. Micromonospora coerulea Jensen. (Jensen, H., Proc. Linnean Soc. N. S. Wales, 57, 1932, 173.)

Vegetative growth: Slow growth on glucose-asparagine agar. Mycelium 
dense, greenish blue. Pigment insoluble. Colonies pigmented only on free admission of oxygen. Surface of colonies hard and glossy.

Aerial growth: Thin, white veil on surface resembling aerial mycelium, but without aerial spores.

Aerial mycelium: None. Spherical conidia produced on branching short conidiophores. Conidia blue.

Liquid media: Growth at bottom, in the form of firm, round, white to pink granules.

Gelatin: Rapid liquefaction.

Milk: Possible coagulation, but digestion very slight.

Starch: Hydrolysis positive.

Cellulose: No decomposition.

Nitrate: No reduction.

Sucrose: No inversion.

Source: Occurs seldom in soil. 


\section{Description of Species of Thermoactinomyces}

1. Thermoactinomyces vulgaris Tsiklinsky. (Tsiklinsky, P., Ann. Inst. Pasteur, 19, 1899, 500.)

Vegetative growth: Good growth on organic and synthetic media. Mycelium $0.5 \mu$ in diameter. No soluble pigment.

Aerial mycelium: White, powdery. Spherical and oval spores are borne singly at the ends of short branches, from which they are easily broken.

Synthetic agar: Growth colorless, covered with white aerial mycelium. Sporophores very short, not exceeding $2 \mu$ in length, often only $0.5-1.0 \mu$. Spores oval or spherical, often appear to sit directly on mycelium.

Nutrient agar: Good growth, producing aerial mycelium.

Potato: Growth good.

Gelatin: Liquefaction positive.

Milk: Coagulation and peptonization.

Starch: Hydrolysis positive.

Cellulose: No decomposition.

Nutrient broth: Growth in form of white pellicle and often as compact balls on bottom of culture.

Nitrate: No reduction.

Sucrose: No inversion.

Temperature: Grows at $48^{\circ}$ to $68^{\circ} \mathrm{C}$; optimum at $57^{\circ} \mathrm{C}$.

Source: Human and animal excreta, high temperature composts, selfheated hay, soil.

2. Thermoactinomyces monospora (Lehmann and Schütze emend. Jensen). comb. nov. (Schütze, H., Arch. Hyg., 67, 1908, 35; after Krassilnikov, 1941.)

Vegetative growth: Good growth in various media; yellowish, compact, smooth or lichnoid. Hyphae about $1.0 \mu$ in diameter.

Aerial mycelium: Well-developed, covering the whole growth, grayish green. Good sporulation of hay infusion-peptone agar; somewhat less on glycerol-peptone and lactose-peptone agar; none on peptone-glucose agar. Oval spores, $1.5-1.8$ by $1.0-1.4 \mu$, produced on simple short sporophores.

Potato: No growth.

Gelatin: Liquefaction positive.

Milk: No coagulation. 
Blood serum: Good, smooth growth; liquefaction positive.

Temperature: Optimum $37^{\circ}-55^{\circ} \mathrm{C}$; grows poorly at $27^{\circ} \mathrm{C}$ and not at all at $60^{\circ} \mathrm{C}$.

Source: Self-heated hay.

3. Thermoactinomyces thalpophilus Waksman and Corke (Waksman, S. A., and Corke, C. T., J. Bacteriol., in press).

Vegetative growth: Good growth on organic and synthetic media, except on potato, at $50^{\circ} \mathrm{C}$. Growth colorless with yellow pigmentation in edges; color changing to orange with age of culture. Soluble pigment wine-colored to light rose produced in yeast-glucose agar.

Aerial mycelium: Well-developed; white, powdery, with tendency to form "fairy rings" on some media. Sporulating hyphae very short, 0.6-1.0 $\mu$ in length. Spherical conidia, $0.8-1.5 \mu$ in diameter, are produced singly on the short sporophores or are entirely sessile.

Synthetic sucrose agar: Abundant colorless growth; powdery white to light-gray aerial mycelium.

Potato: Limited vegetative growth; limited powdery, white aerial mycelium.

Gelatin: Liquefaction positive.

Milk: Coagulation, with limited peptonization.

Starch: Rapid hydrolysis.

Nitrate: Limited reduction to nitrite.

Nutrient-glycerol agar: Cream-colored vegetative growth; white aerial mycelium.

Glucose-asparagine agar: Colorless growth; white, powdery aerial mycelium; reverse of growth white to yellow to light brown. Considerable development of "fairy rings" in aerial mycelium.

Yeast-glucose agar: Good, colorless growth, pigmented yellow at edges, color turning orange in older cultures; lichnoid, white aerial mycelium; soluble wine-colored to rose pigment.

Temperature: No growth at $28^{\circ} \mathrm{C}$; good growth in 4 days at $37^{\circ} \mathrm{C}$; excellent growth in 2 days at $50^{\circ} \mathrm{C}$.

Source: Soil; high temperature composts. 


\section{Description of Species of Nocardia}

Next to species of the streptomycetes, the nocardias are known to be the most prolific producers of antibiotics. Although none of these has so far found any practical application, the possibility is not excluded that in time they might be found useful.

The genus Streptomyces comprises forms producing plant diseases but no animal disease has ever been associated with a true member of this group. The Nocardia group, on the other hand, contains a number of forms known to be associated with animal diseases, or at least to have been isolated from tissues so diseased. This gave origin to a condition known as "nocardiosis."

In contradistinction to the genus Actinomyces, the Nocardia group is aerobic in nature. The colonies are smooth, rough or folded, usually of a soft or dough-like consistency, but often compact and leathery, especially in early stages of growth. Most species do not produce any aerial mycelium. Some produce a limited aerial mycelium which is structurally similar to that of the vegetative mycelium. Some of the species are acid-fast.

1. Nocardia farcinica Trevisan. (Nocard, M. E., Ann. Inst. Pasteur, 2, 1888, 293; Trevisan, V., I generi e le specie delle Batteriacee, Milan, $1889,9)$.

Mycelium: Filaments $0.25 \mu$ thick, branched. Markedly acid-fast.

Nutrient agar: Colonies yellowish white, irregular, refractive, filamentous.

Potato: Growth abundant, dull, crumpled, whitish yellow.

Gelatin: Small, circular, transparent, glistening colonies, No liquefaction. No proteolysis.

Milk: Unchanged.

Starch: No hydrolysis.

Nutrient broth: Clear, with granular sediment, often with gray pellicle.

Nitrates: No reduction.

Temperature: Optimum $37^{\circ} \mathrm{C}$.

Source: Cases of bovine farcy.

Remarks: No soluble pigment formed.

2. Nocardia asteroides (Eppinger) Blanchard. (Eppinger, H. L., Beitr. Pathol. Anat., 9, 1891, 287; Blanchard, in Bouchard, Traité Pathol. Gen., 2, 1895, 811). 
Mycelium: Straight, fine, $0.2 \mu$ thick; breaks up into small, coccoid conidia. Acid-fast.

Synthetic agar: Thin, spreading, orange growth. No aerial mycelium. No soluble pigment.

Nutrient agar: Much-folded, light yellow growth, becoming deep yellow to yellowish red. No soluble pigment.

Potato: Growth much wrinkled, whitish, becoming yellow to almost brick-red.

Gelatin: Yellowish surface growth. No growth in stab. No liquefaction. Milk: Orange-colored ring. No coagulation. No peptonization.

Starch agar: Restricted, scant, orange growth. No hydrolysis.

Glucose broth: Thin, yellowish pellicle.

Nitrate: Reduction to nitrite.

Temperature: Optimum $37^{\circ} \mathrm{C}$.

Source: Cerebral abscess in man.

Pathogenicity: Transmissible to rabbits and guinea pigs but not to mice.

Remarks: A number of strains of acid-fast actinomycetes isolated from human lesions have deviated in certain particulars from the description of Nocardia asteroides, but not sufficiently to warrant separation as species.

3. Nocardia polychromogenes (Vallée) Waksman and Henrici. (Vallée, H., Ann. Inst. Pasteur, 17, 1903, 288; Jensen, H., Proc. Linnean Soc. N. S. Wales, 56, 1931, 79, 363.)

Mycelium: Long wavy filaments, $0.4-0.5$ by $70-100 \mu$, extensively branched but without septa. Older cultures consist entirely of rods 4-10 $\mu$, frequently in $\mathrm{V}, \mathrm{Y}$, or smaller forms. Still older cultures consist of shorter rods and coccoid forms. Gram-positive, frequently showing bands and granules.

Nutrient agar: Scant, orange-red growth.

Glucose agar: After 3 to 4 days raised, flat, glistening, rose-colored growth; after 1 to 3 weeks becoming folded and coral-red.

Gelatin: Growth along stab thin, yellowish, with thin radiating filaments. Surface growth flat, wrinkled, red. No liquefaction.

Milk: Growth starts as small orange-colored surface granules. After 1 to 2 weeks a thick, soft, orange-colored sediment forms.

Glucose broth: After 3 to 4 days turbid; after 2 to 3 weeks an orange flaky sediment. No surface growth.

Temperature: Optimum $22^{\circ}-25^{\circ} \mathrm{C}$.

Source: Blood of a horse; soil in France and Australia.

Remarks: Differs from Nocardia corallina in the formation of very long filaments and in filiform growth in gelatin stabs. 
4. Nocardia opaca (den Dooren de Jong) Waksman and Henrici. (den Dooren de Jong, Centrbl. Bakt. II, 71, 1927, 216.)

Mycelium: Long, curved, irregular and branching filaments or rods, $0.8-1.0$ by $2-16 \mu$, or occasionally longer. Few chains or clumps are formed. In older cultures shorter rods or cocci are generally formed. Readily stained. Not acid-fast. Gram-positive.

Gelatin: Round, convex, whitish, smooth, shining colonies with edges slightly arborescent. Deep colonies: burrs, with slightly irregular processes. Stab: convex, whitish, smooth, resinous, filiform, erose.

Milk: Grayish pellicle; slightly alkaline.

Nutrient broth: Turbid with broken white scum, or clear with granular suspension.

Nitrate: Reduction to nitrite.

Dorset's egg medium: Spreading, smooth, moist, salmon-colored growth.

Loeffler's medium: Scanty growth, smooth, moist, light buff-colored.

Potato glycerol: Dry, rough, crumpled, pink to buff-colored growth.

Temperature: Optimum $30^{\circ} \mathrm{C}$.

Source: Soils in Great Britain.

Remarks: Differs from Nocardia corallina and Nocardia polychromogenes in that the cells are much longer than those of the former and much shorter than those of the latter. Grows in smooth convex surface colonies and burr-like deep colonies. Erikson (J. Gen. Microbiol., 3, 1949, 363) added the following characteristics: Soft cream to pink growth on nutrient agars. On synthetic media, growth colorless and thin, producing an initial mycelium, the hyphae dividing rapidly into short rods; addition of $0.01 \%$ $\mathrm{MnSO}_{4}$ stimulated production of pale pink pigment. Acid-fast cell elements predominated during periods of maximum growth and free air supply.

No acid from sucrose, lactose, maltose, or glucose; phenol and naphthalene are utilized as sources of energy.

5. Nocardia erythropolis (Gray and Thornton) Waksman and Henrici. (Gray, P., and Thornton, H., Centrbl. Bakt. II, 73, 1928, 87.)

Mycelium: Long uneven-sided rods and filaments, curved and branching up to $11 \mu$ long by $0.8 \mu$. Coccoid forms not formed. Stains readily. Not acid-fast. Gram-positive.

Nutrient agar: Round colonies, 2-3 $\mathrm{mm}$ in diameter. convex, watery white; edge entire; deep colonies lens-shaped. Slant: filiform, flat, watery growth; edge undulate.

Gelatin: After 12 days, round, flat, white, shining colonies; edge entire. Deep colonies: round, smooth. Stab: after 8 to 14 days, convex, white, 
smooth, shining, radiate from center, borders cleft. Line of puncture filiform, erose.

Milk: Pale pink pellicle.

Nutrient broth: Growth slight, turbid.

Nitrate: No reduction.

Dorset's egg medium: After 2 weeks raised, moist, finely granular, irregular margin, flesh-colored.

Loeffler's medium: After 7 days, growth as on Dorset's egg medium, but pink.

Potato glycerol: After 7 days, flat, dry, rough, orange-colored.

Phenol is utilized.

Temperature: Optimum $25^{\circ} \mathrm{C}$.

Source: Soils in Great Britain.

Remarks: Differs from Nocardia coeliaca and Nocardia actinomorpha in the filiform growth and absence of liquefaction of gelatin. Long rods and filaments. No acid from glucose, lactose, sucrose, or glycerol.

6. Nocardia leishmanii Chalmers and Christopherson. (Birt and Leishman, J. Hyg., 2, 1902, 120; Chalmers, A., and Christopherson, I., Ann. Trop. Med. Parasitol., 10, 1916, 255.)

Mycelium: Initial cells frequently swollen, large and irregular, aggregated in short chains and then branching out into regular narrow filaments; at margin of colony on synthetic glycerol agar may be seen comparatively long thick segments with accompanying fringe of normal hyphae; later entire colonies asteroid in appearance, very fine and close angular branching, with aerial hyphae situated singly; aerial mycelium generally abundant with irregularly cylindrical conidia. Slightly acid-fast. The latter property must have been attenuated during artificial cultivation, for the organism is reported by the original isolators as markedly acid-fast.

Glucose agar: Rounded elevated colonies with paler frosting of aerial mycelium; growth becoming piled up, aerial mycelium sparse.

Gelatin: Small pink colonies in depths of stab. No liquefaction.

Milk: Surface growth, white aerial mycelium, solid coagulum; later partly peptonized with pink aerial mycelium.

Glycerol agar: Small round pink colonies, tending to be umbilicated and piled up, stiff white aerial spikes.

Potato agar: Minute colorless round colonies, small raised patches of white aerial mycelium.

Dorset's egg medium: Colorless confluent growth studded with little wart-like projections bearing stiff aerial spikes; growth becomes pinkish with white aerial mycelium; later, growth drab-gray, medium discolored. 
Serum agar: Minute, round, colorless colonies with pinkish tinge in confluent raised patch.

Nutrient broth: Liberal growth, white flocculent colonies; later pink surface colonies.

Carrot plug: Small, irregularly round, raised colonies, colorless, covered with stiff aerial spikes; later buff-colored, convoluted, and ribbed growth with small patches of white aerial mycelium; aerial mycelium pink in 2 months.

Source: Fatal case of lung disease and pericarditis in man.

7. Nocardia caprae (Silberschmidt) Waksman and Henrici. (Silberschmidt, Ann. Inst. Pasteur, 13, 1899, 841.

Mycelium: Initial cells only slightly enlarged; early development of aerial hyphae, while substratum threads are still short; frequent slipping of branches; aerial mycelium abundant on all media with tendency to form coherent spikes; mycelium not very polymorphous, but occasional thicker segments appear. Slightly acid-fast.

Glucose agar: Irregular, bright pink growth tending to be heaped up; later abundant masses frosted over with thin, white aerial mycelium.

Potato: Abundant growth, small colonies, mostly confluent, entirely covered with pale pink aerial mycelium; growth becomes membranous, considerably buckled; later superficial colonies with pink aerial mycelium on liquid at base of tube, bottom growth of round white colonies.

Gelatin: Extensive dull growth with small raised patches of pink aerial mycelium; later ribbon-like, depressed. No liquefaction.

Milk: Red surface skin; solid coagulum.

Starch: No hydrolysis.

Glucose broth: Small sediment of fine flocculi; later, pellicle composed of small pink colonies; superficial skin entire and salmon-colored in 16 days.

Nutrient broth: Superficial pellicle composed of pink colonies with white aerial mycelium; moderate flocculent sediment.

Glycerol agar: Abundant growth, small round pink colonies, partly covered with white aerial mycelium.

Potato agar: Extensive, thin growth, pink in raised patches, covered by white aerial mycelium; later aerial mycelium also becomes pink.

Starch agar: Minute colorless colonies covered by white aerial mycelium.

Blood agar: Minute, round, colorless colonies aggregated in broad pink zones. paler aerial mycelium. No hemolysis.

Dorset's egg medium: Few colorless colonies, some pink, white aerial mycelium; later, growth becoming dull pink, irregular, with scant white aerial mycelium. 
Calcium agar: Minute colorless colonies, white aerial mycelium; later a pinkish tinge.

Serum agar: Small, round, pink colonies frosted over with thin, white aerial mycelium.

Synthetic glycerol solution: Round pink disk-like colonies on surface and tenuous white wispy growth in suspension and sediment; after 20 days, surface colonies bearing white aerial mycelium extending $2 \mathrm{~cm}$ up tube.

Source: Lesions in goats.

8. Nocardia pretoriana Pijper and Pullinger. (Pijper, A., and Pullinger, B. D., J. Trop. Med. Hyg., 30, 1927, 153.)

Mycelium: Minute, flat colonies consisting of angularly branched filaments, and bearing a few short straight aerial hyphae; later, growth becomes spreading and extensive, the slipping of the branches is well marked and the aerial hyphae are divided into cylindrical conidia. Slightly acid-fast.

Glucose agar: Pale buff, umbilicated and piled up colonies.

Potato: Small, raised, pale pink colonies with white aerial mycelium; after 2 months, plug and liquid discolored, growth dull buff, dry and convoluted at base, round and zonate at top of slant, white aerial mycelium, surface and bottom growth on liquid.

Gelatin: A few colorless flakes. No liquefaction.

Milk: Yellowish surface growth; solid coagulum in 1 month; later, partly digested, pale pink growth up the wall of the tube.

Nutrient broth: Moderate quantity of flakes and dust-like surface growth.

Glycerol agar: Piled up pink mass, very scant white aerial mycelium at margin.

Dorset's egg medium: A few round, colorless colonies in 3 days; after 3 weeks, irregular, raised pink mass, warted appearance, moderate degree of liquefaction.

Calcium agar: Yellowish wrinkled coherent growth with white aerial mycelium on apices and at margin.

Serum agar: Raised, convoluted, slightly pinkish growth.

Synthetic sucrose solution: A few colorless flakes on the surface, lesser bottom growth.

Source: A case of mycetoma of the chest wall in a South African native.

9. Nocardia vacciniae Demarec and Smith. (Demarec, J. B., and Smith, N. R., Phytopathology, 42, 1952, 249-252.)

Growth: Rods and filaments $0.4-0.8 \mu$ in diameter, granular appearance when stained and eventually breaking up into bacillary forms, few cells acid-fast. Fat was demonstrated by staining with Sudan black B. 
Synthetic agar: Growth scant, gray.

Nutrient agar: Growth poor, slow, granular, gray, sometimes pinkish in old cultures.

Potato: Growth slow, spreading, raised, gray.

Milk: Growth dry, raised, gray with orange spots; no hydrolysis.

Potato-yeast-mannitol agar: Growth abundant, fluffy, gray to orange.

Nitrate: Slow reduction.

Gelatin-nutrient agar: Dry, ribbon growth; no hydrolysis.

Starch-nutrient agar: Dry, ribbon, pinkish to orange growth; hydrolysis positive.

Citrates: Limited utilization.

Paraffin: Utilization.

Temperature: Growth best at $25^{\circ}-28^{\circ} \mathrm{C}$; inhibited at $32^{\circ}$; none or very scant at $37^{\circ}$.

Antibiotic activity: None.

Remarks: On a basal agar with ammonia as the source of nitrogen, acid was formed from glucose, sucrose, glycerol, and mannitol; reactions variable with arabinose and xylose; no growth on lactose or sorbitol. Resembles Nocardia minima (Jensen) Waksman and Henrici, but differs from it in the following respects: Utilizes glycerol and mannitol and sometimes arabinose and xylose; reduces nitrates to nitrites; utilizes citrates; and causes formation of bud-proliferating galls on blueberry plants.

10. Nocardia pulmonalis (Burnett) Waksman and Henrici. (Burnett, S. H., Ann. Rept. N. Y. State Vet. Coll., 1909-1910, 167).

Mycelium: Gram-positive mycelium breaking up readily into oval-shaped conidia. Acid-fast, especially in early stages of growth.

Nutrient agar: Moist, raised growth in form of small, spherical colonies.

Glucose agar: Dull, whitish, convoluted growth.

Potato: Luxuriant growth in form of small, translucent, round colonies; becoming colored lemon-yellow; later, growth becomes convoluted or folded with chalky white aerial mycelium, color of plug brownish.

Gelatin: Small, whitish, spherical colonies; edges of colony becoming chalky white; limited liquefaction.

Milk: Colonies on the surface of the medium; coagulation in a few days; later, digestion.

Nutrient broth: Delicate, translucent film on surface, becoming corrurugated with some whitish, spherical colonies in medium.

Source: Lungs of a cow.

Remarks: Nonpathogenic for rabbits and guinea pigs. 
11. Nocardia paraffinae (Jensen) Waksman and Henrici. (Jensen, H., Proc. Linnean Soc. N. S. Wales, 56, 1931, 362.)

Mycelium: In agar media, the organism initially forms an extensive mycelium of long, richly branching hyphae, $0.4-0.5 \mu$ thick. After 5 to 6 days, at room temperature, numerous end branches swell to about double thickness, become more refractive, exhibit fine incisions along their external contours, and divide into oval, spore-like elements, $0.8-1.0$ by $1.2-$ $1.5 \mu$. This process of division starts at the tips of the swollen branches and proceeds basipetally until most of the hyphae appear divided. Primary septa have not been seen in the hyphae. A similar process of division takes place in liquid media, where also the filaments often fall into fragments of variable length. The spore-like elements, but not the undivided filaments, are markedly acid-fast. The aerial mycelium consists of rather short, straight, not very much branched hyphae, $0.4-0.6 \mu$ thick, which never show any differentiation into spores. Erikson (loc. cit.) added the following characteristics: Growth hard, firm, yellowish, with a certain amount of white aerial mycelium on most nutrient agars. Because of the latter property, this organism is said to approach the true Streptomyces types. Acidfastness is observed in certain stages, notably in the early stages of growth and in the coccus forms.

Nutrient agar: Slow but good growth. Vegetative mycelium superficial, somewhat raised, ocher-yellow, hard, but with a loose, smeary surface. Aerial mycelium scant, small white tufts. No pigment.

Glucose agar: Fair growth. Vegetative mycelium flat, growing into medium; pale ocher-yellow to orange, with raised outgrowths on the surface. Growth of a crumbly consistency. Scant, white aerial mycelium.

Potato: Fair growth. Vegetative mycelium granulated, first pale yellow, later deep ocher-yellow to orange. Scant, white aerial mycelium. No pigment.

Gelatin: No liquefaction.

Milk: No coagulation or digestion.

Starch: No hydrolysis.

Cellulose: No decomposition.

Nitrate: No reduction.

Sucrose: No inversion.

Sucrose agar: Very scant growth. Thin colorless veil, sometimes with a trace of white aerial mycelium.

Liquid media (milk, broth, synthetic solutions): Small, round granules of various yellow to orange colors, firm but can be crushed into a homogeneous smear. In old broth cultures a thick, hard, orange to brownish surface pellicle is formed. 


\section{Habitat: Soil.}

Remarks: All strains show a marked power of utilizing paraffin wax as a source of energy.

12. Nocardia minima (Jensen) Waksman and Henrici. (Jensen, H., Proc. Linnean Soc. N. S. Wales, 56, 1931, 365.)

Mycelium: Filaments and rods $0.4-0.6$ by $2-10 \mu$. In older cultures mostly short rods, frequently V, Y, swollen forms, or cocci. Irregularly stained with ordinary dyes, generally show bars and bands. Generally a few cells from cultures are acid-fast, most are not acid-fast. Gram-positive.

Nutrient agar: Slow growth, raised, folded, with finely myeloid margins. At first colorless, after 6 to 8 weeks flesh pink or coral pink.

Potato: Growth slow, after 6 to 8 weeks abundant, spreading, much raised, finely wrinkled, coral-pink.

Gelatin: Filiform, granulated, cream-colored growth. No liquefaction.

Paraffin: Utilized.

Temperature: Optimum $22^{\circ}-25^{\circ} \mathrm{C}$.

Habitat: Soil.

Remarks: Closely resembles Nocardia corallina but differs in the much slower growth and the smaller size of the cells.

13. Nocardia corallina (Bergey et al.) Waksman and Henrici. (Hefferan, M., Centrbl. Bakt. II, 11, 1904, 459; Bergey et al., Manual, 1st ed., 1923, 93.)

Mycelium: Branching rods, generally curved, $1-1.5$ by $3-10 \mu$. In older cultures generally shorter rods and cocci. Nonmotile. Not acid-fast. Gram-positive.

Nutrient agar: Round, convex, or umbonate colonies, smooth, pink, shining or matte; border lighter, edge filamentous or with arborescent projections. Deep colonies: burrs, or lens-shaped, with arborescent projections. In their very early stages colonies consist of branching filamentous rods. As the colony grows, the cells in the interior break up into short rods and cocci which eventually form the mass of the colony. Cells on the outside remain filamentous, giving the colony a burr-like appearance, and often forming long arborescent processes. Slant: filiform, convex, smooth, pink, shining or matte: arborescent or with projections from undulate border.

Gelatin: Round, convex, smooth, pink, shining colonies, edge filamentous. Deep colonies: burrs. Nailhead stab: line of stab arborescent. No liquefaction.

Milk: Alkaline. Reddish pellicle.

Nutrient broth: Usually turbid. Pink scum. 
Nitrate: Reduction to nitrite.

Dorset's egg medium: Filiform, raised, dry, wrinkled, orange.

Loeffler's medium: Similar to growth on Dorset's egg medium, but pink.

Potato glycerol: Filiform, raised, dry, wrinkled, yellowish brown to coralred.

Temperature: Optimum $22^{\circ}-25^{\circ} \mathrm{C}$.

Habitat: Soil.

Remarks: Acid from glycerol and glucose with some strains. No acid or gas from sucrose, maltose, or lactose. Phenol and $m$-cresol are utilized. Some strains utilize naphthalene (Gray and Thornton).

14. Nocardia globerula (Gray) Waksman and Henrici. (Gray, P., Proc. Roy. Soc. [London] B, 102, 1928, 265.)

Mycelium: Curved rods and filaments 1 by $2-9 \mu$, with many coccoid cells, especially in old cultures. Rods and filaments frequently irregularly swollen. Not acid-fast. Capsules may be present. Gram-positive.

Nutrient agar: After 4 days surface colonies irregularly round, 3-5 mm in diameter, convex, white, smooth, shining; edge undulate, erose. After 7 days, more convex and of a watery appearance. Deep colonies: after 4 days, lens-shaped. Slant: after 3 days, filiform, flat, watery; edge irregular.

Gelatin: After 19 days, surface colonies irregularly round, 1-2 $\mathrm{mm}$ in diameter, convex, light buff, smooth, shining; edge entire. Deep colonies: round, with entire edge. Stab: after 8 days, nailhead, irregularly round, convex, pinkish white, smooth, shining; line of stab erose.

Milk: Alkaline.

Nutrient and peptone broth: Turbid with viscous suspension.

Nitrate: No reduction.

Dorset's egg medium: After 2 weeks, spreading, raised, mojst, orangecolored.

I Loeffler's medium: Growth as on Dorset's egg medium, but salmoncolored.

Potato glycerol: After 24 hours, filiform, moist, smooth, pale pink.

Indole agar: Blue crystals of indigotin formed.

Temperature: Optimum $25^{\circ}-28^{\circ} \mathrm{C}$.

Phenol: Utilized.

Source: Soil in Great Britain.

Remarks: This organism resembles most closely Nocardia corallina. It is distinguished by producing a more watery type of surface growth, more nearly entire deep colonies, and more particularly by the production of indigotin from indol. No acid from glucose, lactose, maltose, sucrose, or glycerol. 
15. Nocardia salmonicolor (den Dooren de Jong) Waksman and Henrici. (den Dooren de Jong, Centrbl. Bakt. II, 71, 1927, 216.)

Mycelium: On glucose-asparagine agar after 18 to 24 hours, long branching rods are formed, 1.0-1.3 $\mu$ in thickness, with small refractive granules of aerial mycelium, sometimes stretching into quite long filaments; after 2-3 days, small definite mycelia are present, and after 5-6 days these have largely divided into short rods and cocci; the colonies have the same burrlike appearance as those of Nocardia corallina. Many cells at the edge of the colonies show, after 3-4 days, club- or pear-shaped swellings, up to 2.5-3.0 $\mu$ in width; after 5-6 days, many of these swollen cells germinate with the formation of two more slender sprouts. Erikson added the following characteristics. Rich salmon-pink to yellow-pigmented soft growth on nutrient agar. Fair to good growth on simple media. A few short, undivided aerial hyphae appear on some media, which may actually form a thin white frosting over the pink growth. Acid-fastness is found among the earlier stages of growth, especially in some of the strains and on some media.

Glucose-nutrient agar: Excellent growth, spreading, flat, dense, edges lobate, surface folded, glistening, yellow, gradually changing to deep orange-red.

Potato: Good growth, raised, warty, crumbly, glistening, at first buff, changing to orange and finally to almost blood-red.

Gelatin: At $20-22^{\circ} \mathrm{C}$, scant arborescent growth in stab; small, wrinkled, orange surface colony. No liquefaction.

Milk: Good growth; pellicle of small cream-colored granules after 2 days, later a thick orange sediment. Not coagulated, but appears slightly cleared after 5 weeks, the reaction becoming alkaline.

Starch: No hydrolysis.

Nutrient broth: Fair growth; thin pellicle and granular sediment, at first cream-colored, later red; broth clear at first, slightly turbid after 3 weeks.

Nitrate: Reduction to nitrite. Nitrate, ammonium salts, asparagine, and peptone are utilized almost equally well with glucose as source of carbon, although the growth is most rapid with peptone.

Sucrose: No inversion, although readily utilized with sodium nitrate as a source of nitrogen.

Glucose-asparagine agar: Good growth, restricted, rather flat, edges lobate, surface warty, glistening, first pale orange, later ocher-yellow; consistency crumbly. After 5 to 6 weeks, the growth is paler with many small, round, raised, yellow secondary colonies.

Paraffin: Readily utilized as a source of carbon.

Phenol: Not utilized.

Source: Probably soil.

Remarks: Closely related to Nocardia corallina. 
16. Nocardia rubropertincta (Hefferan) Waksman and Henrici. (Grassberger, R., Münch. Med. Wochschr., 46, 1899, 343; Hefferan, M. Centrbl. Bakt., II, 11, 1904, 460.)

Mycelium: Small rods $0.3-0.9$ by $1.5-3.0 \mu$. Cells in $18-24$ hour agar culture in beautiful angular arrangement, after 2-3 days nearly coccoid, 0.6 by $0.8 \mu$. Tendency for branching on glycerol agar after $2-3$ days, but branching does not occur commonly, though granules of aerial mycelium are sometimes seen (Jensen). Not acid-fast (Grassberger). Acid-fast (Hefferan). Variable (Jensen). Nonmotile. Gram-positive.

Nutrient agar: Small granular colonies becoming pink to red depending on composition of agar. Slant: Dry, lusterless (R) to glistening (S), pink to vermillion-red.

Potato: Slow but excellent intensive red growth becoming dull orange (Jensen).

Gelatin: Irregular colonies with crenate margin and folded surface. Coralred. Growth in stab at first thin, then granular to arborescent with chromogenesis. No liquefaction.

Milk: Thick, fragile, dull coral-red surface scales and sediment. Unchanged (Hefferan) to alkaline and somewhat viscid after 3-4 weeks (Jensen).

Nutrient broth: Faint uniform turbidity with salmon-pink pellicle (in scales) which is renewed on surface as it settles to form a red sediment (Hefferan, Jensen).

Nitrate: No reduction to nitrite. Nitrates, ammonia, and asparagine are almost as good sources of nitrogen as peptone (Jensen).

Temperature: Grows well between $20^{\circ}$ and $37^{\circ} \mathrm{C}$ (Jensen).

Aerobic to facultative anaerobe.

Source: Six cultures isolated from butter. Several cultures isolated from soil in Holland and Australia. Two cultures as contaminants in tuberculin flasks.

Remarks: Mycobacterium-like colonies with coral to vermillion-red chromogenesis on asparagine agar, potato, gelatin, and other media. Short rods, seldom forms filaments. Generally not acid-fast.

Benzine, petroleum, paraffin oil, and paraffin are utilized as sources of energy. No action on manganese dioxide (Söhngen, N. L., Centrbl. Bakt. II, 40, 1914, 554).

17. Nocardia rubra (Krassilnikov) comb. nov. (Krassilnikov, N. A., Actinomycetales, Akad. Nauk. USSR, Moskau, 1941, 81.)

Vegetative growth: Threads at first filamentous, developing into a unicellular mycelium; after a few days, frequent septae are produced and the mycelium breaks up into short, rod-shaped, and later coccoid elements. 
These grow into a mycelium on a fresh substrate. Cells are gram-positive, non-acid-fast. Colonies usually rough. Some are smooth, dry, powdery, or dough-like in consistency. Part of mycelium grows into substrate. Colonies pigmented bright red. Pigment not dissolved into medium, weakly soluble in ether, alcohol, and acetic acid; soluble in chloroform; belongs to the carotinoids.

Aerial mycelium: None produced.

Synthetic agar: Good typical growth.

Nutrient agar: Poor growth.

Potato: Good typical growth.

Gelatin: No liquefaction.

Milk: No change.

Starch: No hydrolysis.

Cellulose: No growth.

Nutrient broth: Sediment and surface ring; medium clear.

Nitrate: No reduction.

Sucrose: No inversion.

Paraffin: Good growth.

Fats: Well assimilated.

Source: Soil.

Remarks: Grows well in high salt concentrations (5-10 per cent $\mathrm{NaCl}$ ). Krassilnikov considers Nocardia corallina, Nocardia salmonicolor and $\mathrm{No}$ cardia opaca as forms of Nocardia rubra.

18. Nocardia coeliaca (Gray and Thornton) Waksman and Henrici. (Gray, P., and Thornton, H., Centrbl. Bakt. II, 73, 1928, 88.)

Mycelium: Short, curved, uneven-sided rods 0.8 by $5 \mu$ with occasional filaments up to 10-12 $\mu$ long, frequently beaded, occasionally swollen or branched. Coccoid forms $0.8-1.2 \mu$ in diameter are common, especially in older cultures. Stain readily. Not acid-fast, or occasionally slightly acid-fast. Gram-positive.

Nutrient agar: After 11 days, colonies less than $1 \mathrm{~mm}$ in diameter, round or irregular, raised, white, resinous, edge irregular, burred. Deep colonies: irregularly round or oval, edge slightly broken. Slant: filiform, convex, white, rugose, resinous, edge undulate.

Gelatin: After 12 days, irregular, raised, white, rugose, dull colonies, edge entire. Deep colonies: irregular, smooth or slightly broken. Stab: convoluted, buff-white to yellowish, dull; below surface the growth forms many irregular hollow lobes, giving a glistening appearance, to a depth of 3-4 mm.

Milk: Slightly alkaline after 5 to 7 days.

Nutrient broth: Turbid. 
Nitrate: No reduction.

Dorset's egg medium: Raised, smooth, moist, verrucose, buff-colored. Loeffler's medium: After 10 days, slight growth, dry, granular, pale buff-colored.

Potato glycerol: After 2 days, dry, crumpled, orange-colored, becoming brown after about 10 days.

Temperature: Optimum $22^{\circ}-25^{\circ} \mathrm{C}$.

Source: Soil in Great Britain and Australia.

Remarks: No acid from glucose, lactose, sucrose, or glycerol. Phenol is utilized. Differs from the previously described members of the genus in the absence of chromogenesis. Forms hollow lobes in deep gelatin cultures. Cells are rods, seldom filaments.

19. Nocardia transvalensis Pijper and Pullinger. (Pijper, A., and Pullinger, B. D., J. Trop. Med. Hyg., 30, 1927, 153.)

Mycelium: Initial unicellular, but with the central branch frequently broader and showing dense granular refractile contents, small colonies quickly covered with aerial mycelium, the straight aerial hyphae in some cases becoming clustered into irregular spikes, colorless drops are exuded and a pink coloration produced in the densest part of the growth on synthetic glycerol agar. Angular branching with division of the substratum filaments can be seen, the aerial hyphae also being irregularly segmented. Acid-fast.

Nutrient agar: No growth.

Glucose agar: Raised, granular, pink colonies with white aerial mycelium.

Potato: Dry, raised, convoluted, pink growth with white aerial mycelium in 1 month; dull, pink, brittle surface colonies, with paler aerial mycelium floating coherently on liquid at base in 2 months.

Gelatin: Poor growth, a few irregular colorless flakes. No liquefaction. Milk: No change.

Starch: No hydrolysis.

Nutrient broth: Moderate flaky growth.

Glycerol agar: Small, pink coiled masses with thin, white aerial mycelium.

Coon's agar: Colorless growth with liberal white aerial mycelium.

Dorset's egg medium: Small, irregularly raised, and coiled, dull pink mass.

Serum agar: Very poor growth.

Synthetic sucrose solution: Poor growth, a few flakes on surface, a few at bottom.

Inspissated serum: Scant, colorless, flaky growth; later a minute tuft of pale pink aerial mycelium.

Source: A case of mycetoma of the foot in South Africa. 
20. Nocardia mesenterica (Orla-Jensen) Waksman and Henrici. (OrlaJensen, S., The lactic acid bacteria, 1919, 181; Jensen, H. L., Proc. Linnean Soc. N. S. Wales, $57,1932,373$.

Mycelium: Extensive, composed of richly branching hyphae of a somewhat variable thickness, $0.4-0.8 \mu$; no aerial hyphae are seen. With increasing age the hyphae divide into fragments of varying sizes and shapes, partly diphtheroid rods, but no real cocci. There is, particularly in richer media, a tendency to form large, swollen, fusiform to almost spherical cells, up to $3.5 \mu$ in diameter. These may stain intensely with carbol fuchsin; when transferred to fresh media, they germinate and produce a new mycelium.

Glucose-nutrient agar: Good growth, restricted, with undulate edges, surface with high transverse folds, cream-colored; the consistency is firm and cartilaginous after 2 days, later looser and more brittle. Growth at $28^{\circ}$ to $30^{\circ}$ rather scant; smooth, soft, glistening, cream-colored smear.

Potato: Scant growth; restricted, soft, cream-colored smear.

Gelatin: Good growth; finely arborescent, cream-colored growth in the stab; raised, folded, pale yellow, surface colony. No liquefaction.

Milk: At $28^{\circ}$ to $30^{\circ} \mathrm{C}$, small cream-colored granules along the tube; the milk undergoes no visible changes within 4 weeks. No proteolytic action.

Starch: Hydrolysis.

Cellulose: No decomposition.

Nutrient broth: Good growth; voluminous, flaky, whitish sediment; broth clear.

Nitrate: No reduction.

Sucrose: Inversion.

Glucose-asparagine agar: Fair growth, narrow, raised, granular, very pale yellow, glistening; condensation water-clear, with small granules. At $30^{\circ} \mathrm{C}$ only scant growth consisting of small irregular white granules, growing deeply into the agar.

Sabouraud's agar: Excellent growth, spreading, at first flat and smooth, pale straw-yellow, perfectly hard and cartilaginous, later raised and strongly folded, of a loose, curd-like consistency, bright lemon-yellow. Growth at $28^{\circ}$ to $30^{\circ} \mathrm{C}$ only fair, restricted, folded, cream-colored, soon becoming soft and smeary.

Source: Fermented beets.

Remarks: Sodium nitrate, ammonium phosphate, and asparagine utilized, although these are inferior to peptone as sources of nitrogen.

21. Nocardia albicans (Krassilnikov) comb. nov. (Krassilnikov, N. A., Actinomycetales, Akad. Nauk. USSR, Moskau, 1941, 71.) 
Vegetative growth: On solid media, hyphae break up into rod-shaped cells, $12-25$ by $0.6-0.7 \mu$, up to 50 in length. Cells straight or slightly curved, branching, gram-positive.

Aerial mycelium: None observed, except surface layer of sporophores, which produce a velvety appearance. Multiplication by fission, seldom by budding.

Nutrient agar: Good growth; smooth, shiny colonies.

Gelatin: No liquefaction.

Milk: No change.

Starch: Hydolysis.

Cellulose: No growth.

Nutrient broth: Poor growth; produces faint turbidity, which settles on bottom and leaves a surface ring. No true mycelium produced. Cells rodshaped 5-10 $\mu$, seldom $15-20 \mu$.

Nitrate: No reduction.

Sucrose: Inversion.

Source: Soil.

Remarks: Uses glycerol well, but not paraffin. Uses nitrate as source of nitrogen.

22. Nocardia flava (Krassilnikov) Waksman and Henrici. (Krassilnikov, N. A., Bull. Acad. Sci. USSR, No. 1, 1938, 139.)

Mycelium: Cells at first filamentous, $0.7-0.8 \mu$ in diameter; after 2-3 days broken into long rods and then into cocci $0.7 \mu$ in diameter. No spores, some strains form chlamydospores. Cell multiplication by fission, cross-wall formation, rarely by budding. Cells gram-positive; not acid-fast.

Synthetic agar: Bright yellow or gold-colored colonies.

Nutrient agar: Pigment bright yellow or gold color on synthetic media, dirty yellow on meat-peptone media. Pigment not soluble in medium. Surface of colony somewhat shiny or rough and folded, of a dough-like consistency.

Gelatin: No liquefaction.

Milk: No peptonization or coagulation.

Starch: Hydrolysis.

Sucrose: Weak inversion.

Meat peptone: Dirty yellow pigmentation.

Habitat: Soil, not common.

Remarks: No growth on paraffin or wax; weak growth on fat.

23. Nocardia viridis (Krassilnikov) Waksman and Henrici. (Krassilnikov, N. A., Bull Acad. Sci. USSR, No. 1, 1938, 139.) 
Growth: Colonies colored dark green. Pigment not soluble in medium, in water, or in organic solvents. Surface of colony somewhat shiny. On potato, rough, much-folded, broken up into small colonies.

Mycelium: Cells often branching, $0.7-0.8 \mu$ in diameter with cross-wall; after 5-7 days the cells break up into rods, 5-15 $\mu$ long. Cocci not observed. Cells multiply by fission, seldom by budding. Spores not formed. Cells gram-positive, not acid-fast.

Gelatin: No liquefaction.

Milk: No peptonization or coagulation.

Starch: Weak hydrolysis.

Sucrose: Ready inversion.

Habitat: Soil.

Remarks: Good growth on fats and paraffin and less on wax.

24. Nocardia citrea (Krassilnikov) Waksman and Henrici. (Krassilnikov, N. A., Bull. Acad. Sci. USSR, No. 1, 1938, 139.)

Mycelium: In young cultures, very fine threads $0.3-0.5 \mu$ in diameter. After several days the cells break up into short rods 0.5 by $1.5-5 \mu$ and into cocci $0.3-0.5 \mu$ in diameter. Multiplies by fission and bud formation; spores not formed. Cells not acid-fast.

Growth: Yellow-green, usually rough and folded.

Gelatin: Liquefaction.

Milk: Coagulation and peptonization.

Starch: Hydrolysis.

Sucrose: Inversion.

Habitat: Soil and water.

Remarks: Weak growth on fat. No growth on paraffin or wax.

25. Nocardia madurae (Vincent) Blanchard. (Vincent, H., Ann. Inst. Pasteur, 8, 1894, 129; Blanchard, R., in Bouchard, Traité Pathol. Gén. 2, 1896, 868.)

Mycelium: Morphology in tissues, growth in form of granules consisting of radiating actinomycosis. In cultures, initial branched mycelium fragmenting into rod-shaped and coccoid bodies. No aerial mycelium or spores. Not acid-fast.

Potato: Wrinkled friable growth; buff-colored, sometimes red.

Gelatin: Growth scant, whitish; no liquefaction. Colonies round, glistenng, at first white, then buff to rose or crimson. Pigment production irregular and unpredictable. Occasionally red soluble pigment is produced. Growth eventually wrinkled. No aerial mycelium.

Milk: No change, or slight, slow peptonization.

Nutrient broth: Growth as a floccular sediment. 
Source: Cause of some cases of Madura foot.

Remarks: Not pathogenic for the usual laboratory animals; pathogenic for monkeys (Musgrave, W. E., and Clegg, M. T., Philippine J. Sci., Ser. B., Med. Sci., S, 1908, 470).

26. Nocardıa nigra (Krassilnikov) comb. nov. (Not N. nigra Castellani and Chalmerson, not Streptothrix nigra Rossi Doria.) (Krassilnikov, N. A., Actinomycetales, Akad. Nauk. USSR, Moskau, 1941, 89.)

Vegetative growth: Colonies rough, folded, shiny, dough-like consistency. Growth at first colorless or brownish, gradually becoming darker and after 10-15 days, dark brown and even black. Pigment not dissolved in medium. It is similar to melanin. Hyphae thread-like breaking up into rods $2-10$ by $0.7 \mu$ and cocci, $0.6-0.8 \mu$. No aerial mycelium.

Synthetic agar: Growth poor. Many cells are swollen to $3 \mu$ in diameter.

Nutrient agar: Growth poor.

Potato: Good growth.

Gelatin: No growth or liquefaction.

Milk: No change.

Cellulose: No growth.

Nutrient broth: Small sediment produced. Medium clear.

Source: Soil.

Remarks: Seldom found. Culture rapidly disappears on continued cultivation.

27. Nocardia lutea Christopherson and Archibald. (Christopherson, and Archibald, Lancet, 2, 1918, 847.)

Mycelium: Initial elements swollen and segmented, giving rise to irregular, spreading, polymorphous colonies composed of cells of all shapes and sizes with markedly granular contents. Later more monomorphous, the filaments being arranged in angular apposition. Sometimes (e.g., on synthetic glycerol agar) the segments are so granular as to appear banded. On potato agar, small filamentous colonies are formed with irregular angular branching and bear a few isolated short, straight, aerial hyphae.

Nutrient agar: Abundant, coherent, moist, pink membranous growth with round discrete colonies at margin; after 3 weeks, colorless fringed margin, round confluent portion.

Glucose agar: Scant, reddish, smeary growth.

Potato: Carrot-red, moist, thick, granular growth in bands, partly raised and with discrete round colonies; sparse, colorless, very thin aerial mycelium at top of slant in 2 months.

Gelatin: Pale pink wrinkled growth on wall of tube and colorless punctiform and stellate colonies in medium; no liquefaction. 
Milk: Orange-red surface and bottom growth, liquid blue.

Glucose broth: Abundant, pinkish flaky surface growth, breaking up easily and sinking to bottom.

Nutrient broth: Pink flakes and surface growth.

Glycerol agar: Yellowish pink, wrinkled membrane.

Potato agar: Coherent, pink, moist growth, centrally embedded with small, round, discrete colonies at margin.

Dorset's egg medium: Poor growth, dull pink, spreading.

Serum agar: Confluent granular pink membrane.

Source: Actinomycosis of the lachrymal gland.

28. Nocardia blackwellii (Erikson) Waksman and Henrici. (Erikson, D., Med. Research Council Spec. Rept. Ser. 203, 1935, 37.)

Mycelium: Initial elements short, rod-like, growing out into longer forms sparsely branching; small radiating colonies are produced with short, straight aerial mycelium, frequently large, round or ovoid cells are interposed in the irregularly segmented chains of cells, being sometimes isolated in company with 2 or 3 short filaments and sometimes terminal.

Nutrient agar: Confluent wrinkled growth with small, round, pinkish, discrete colonies at margin.

Glucose agar: Abundant, pale pink growth, small conical colonies, piled up, convoluted.

Gelatin: Few colorless, minute colonies along line of inoculation; after 30 days, abundant colorless colonies to $10 \mathrm{~mm}$ below surface, larger pinkyellow surface colonies with white aerial mycelium; no liquefaction.

Milk: Heavy, convoluted, bright yellow surface pellicle; no coagulation.

Nutrient broth: Flakes, later innumerable minute colonies, some adhering to wall just above liquid level.

Glycerol agar: Extensive, granular, irregular, thin, pinkish growth; after 40 days, a few discrete colonies with depressed margins, center piled up, pink.

Potato agar: Small, round, colorless colonies covered with white aerial mycelium; after 2 weeks, colonies dull pink, submerged margins, few aerial spikes, moderate aerial mycelium at top of slant.

Serum agar: Smooth, cream, umbilicated colonies, with submerged growth extending into medium in scallops $5-8 \mathrm{~mm}$ deep; a pale pink mass in 2 weeks.

Carrot plug: Small, round, smooth, cream-colored elevated colonies in 10 days; sparse, stiff, colorless aerial spikes in 16 days; abundantly piled up, convoluted, ocherous growth in 25 days.

Source: Hock joint of foal. 
29. Nocardia cuniculi Snijders. (Snijders, Geneesk. Tijdsch. Ned. Indie, 64, 1924, 47, 75.)

Mycelium: Large swollen cells give rise to ramifying filaments or to small chains of short, thick segments which branch out into more regular hyphae; sometimes the irregular elements are beset with spiny processes before giving rise to typical long, branching filaments; later the picture becomes more monomorphous, and short straight aerial hyphae are borne, which presently exhibit irregular segmentation.

Nutrient agar: Small, round, elevated, cream-colored colonies, umbilicated and radially wrinkled.

Glucose agar: Minute, colorless colonies; becoming dull pink, partly confluent and piled up, few stiff, pink aerial spikes.

Potato: Coral-pink, dry, granular growth, covered to a considerable extent with white aerial mycelium, piled up in center, discrete colonies at margin, pink surface pellicle on liquid and colorless colonies at base.

Gelatin: Few flakes. No liquefaction.

Milk: Heavy yellow growth attached to walls; solid coagulum in 1 month.

Nutrient broth: Small and larger cream-colored, scale-like surface colonies, abundant, flocculent bottom growth.

Glycerol agar: Small, round, elevated, cream-colored colonies, margins depressed; becoming smooth, discrete, yellowish.

Dorset's egg medium: Scant, pinkish, smeary growth.

Serum agar: Small, raised, cream-colored colonies, becoming confluent and piled up.

Source: Infected rabbits.

30. Nocardia rangoonensis (Erikson) Waksman and Henrici. (Erikson, D., Med. Research Council Spec. Rept. Ser. 203, 1935, 37.)

Mycelium: Swollen, round initial cells, giving rise to branching hyphae which segment and present slipping and angular arrangement; few short, straight aerial hyphae, which later develop into a profusely branching, long, waving aerial mycelium. Non-acid-fast.

Nutrient agar: Round, lobate, umbilicated, raised, cream-cololed to pale pink colonies; later, medium discolored dark brown, colonies colorless.

Glucose agar: Convoluted, coherent, cream-colored growth, medium discolored. After 23 days, wrinkled, biscuit-colored growth, colorless margin, border white aerial mycelium, medium dark brown.

Gelatin: Abundant, minute colonies in depths and larger, cream-colored ones on surface with white aerial mycelium; brown pigment surrounding growth. No liquefaction.

Milk: Coagulation, yellow surface ring, becoming partly peptonized; liquid discolored dark brown; brownish growth up side of tube. 
Nutrient broth: Abundant, colorless growth; flocculent mass at bottom and pellicle at surface; medium slightly discolored.

Glycerol agar: Dull, mealy, pink, wrinkled growth; scant, white aerial mycelium at top; medium slightly discolored.

Potato agar: Small, round, lemon-colored colonies, partly confluent, with white aerial mycelium; later medium discolored light brown; submerged growth greenish.

Dorset's egg medium: Extensive, colorless growth, pale pink aerial mycelium in center; later covered with a powdery, pinkish white aerial mycelium.

Serum agar: Colonies irregular, small, elevated, cream-colored, frequently umbilicated.

Carrot plug: Small, round, colorless colonies, velvety white aerial mycelium; in 2 months, piled-up, pink, granular mass with warted prominences; marginal zone white aerial mycelium and thin all-over central aerial mycelium.

Source: Human pulmonary case of streptothricosis.

31. Nocardia caviae Snijders (Snijders, Geneesk. Tijdschr. Ned. Indie, $64,1924,47,75)$.

Mycelium: Initial segmentation, producing elements of approximately even thickness arranged in angular apposition, and later, long, profusely ramifying threads with strongly refractile protoplasm. Aerial mycelium straight and branching, the aerial hyphae with occasional coiled tips divided into cylindrical conidia.

Glucose agar: Piled-up, convoluted, cream-colored to pale pink growth; white aerial mycelium.

Potato: Small, colorless colonies; white, powdery aerial mycelium; later, abundant, raised, pale pink, confluent growth, discolored plug; after 2 months, raised, buckled, pink colonies with white aerial mycelium floating on liquid at base.

Gelatin: A few colorless flakes. No liquefaction.

Milk: Colorless surface growth: white aerial mycelium; coagulation.

Nutrient broth: Cream-colored, wrinkled surface pellicle extending up wall and breaking easily; moderate bottom growth, flaky; medium discolored.

Glycerol agar: Scanty growth.

Potato agar: Colorless, spreading growth with dense, white aerial mycelium.

Dorset's egg medium: Heavily corrugated, pale pink growth with submerged margin, dense white aerial mycelium in center; after 3 weeks, colorless transpired drops. 
Serum agar: Pale pink, wrinkled growth, partly submerged; after 4 weeks, piled up with scant, white aerial mycelium, medium discolored reddish brown.

Source: Infected guinea pigs, Sumatra.

32. Nocardia actinomorpha (Gray and Thornton) Waksman and Henrici. (Gray, P., and Thornton, H., Centrbl. Bakt. II, 78, 1928, 88.)

Mycelium: Long, branching filaments and rods, $0.5-0.8$ by up to $10 \mu$. In older cultures, rods 2-3 $\mu$ long generally predominate. On some media, extensively branching hyphae occur. Readily stained. Not acid-fast. Grampositive.

Nutrient agar: After 11 days, round colonies, $1 \mathrm{~mm}$ in diameter, convex, white, granular or resinous; long arborescent processes from the edge. Deep colonies: arborescent burrs; processes about equal to diameter of colony. Slant: filiform, raised to convex, white, rugose, dull; edge undulate, with strong tufted projections below surface.

Gelatin: After 12 days, round, saucer-like, white colonies, raised rim, edges burred. Liquefaction. Deep colonies: burrs. Stab: after 8 to 14 days, saccate liquefaction, $5-8 \mathrm{~mm}$.

Nutrient broth: Turbid, or clear with white scum.

Nitrate: Reduction.

Dorset's egg medium: After 2 weeks, raised, dry, smooth, salmon-buff growth.

Loeffler's medium: After 2 days, smooth, moist, warty, salmon-colored growth.

Potato glycerol: After 2 days, dry, wrinkled, pink to orange growth. Temperature: Optimum $25^{\circ}-30^{\circ} \mathrm{C}$.

Source: A few strains have been isolated from soil in Great Britain and Australia.

Remarks: Differs from Nocardia coeliaca in saccate liquefaction of gelatin. Long rods and filaments. No acid from glucose, lactose, sucrose, or glycerol. Phenol and naphthalene are utilized.

33. Nocardia alba (Krassilnikov) comb. nov. (Krassilnikov, N. A., Actinomycetales, Akad. Nauk. USSR, Moskau, 1941, 73.)

Vegetative growth: Colonies white, never pigmented, dough-like consistency; smooth or folded growth; shiny or pale. True substrate mycelium produced at first, which breaks up into short rods 2.7 by $0.7-0.8 \mu$, later changing into a mass of coccus-like cells, $0.7-1 \mu$ in diameter. Cells grampositive, non-acid-fast. Many cells are swollen, others form side buds. No aerial mycelium. 
Synthetic agar: Cultures use inorganic sources of nitrogen and sugar, starch, or organic acids as sources of carbon.

Nutrient agar: Good growth.

Gelatin: Liquefaction positive.

Milk: Coagulation and peptonization.

Starch: Rapid hydrolysis.

Cellulose: No growth.

Nitrate: No reduction.

Sucrose: Inversion.

Source: Soil.

Remarks: Several subspecies listed: Nocardia chromogena, Nocardia alba lactica, Nocardia diastaticus.

34. Nocardia flavescens (Jensen) Waksman and Henrici. (Jensen, H. Proc. Linnean Soc., N. S. Wales, 56, 1931, 361.)

Mycelium: On media where a firm growth is produced, the vegetative mycelium appears as long, branched, nonseptate hyphae, 0.4-0.6 $\mu$ thick. In other media, as on nutrient agar and potato, septa are formed and the mycelium appears in preparations as fragments of very variable size, partly resembling highly branched mycobacteria. In several cases-for instance, on nutrient agar at $28^{\circ}-30^{\circ} \mathrm{C}$, in 5 - to 6 -week-old cultures in glucose broth, and in glucose $\mathrm{NH}_{4} \mathrm{Cl}$ solution-short elements assume swollen, fusiform to lemon-shaped forms. The aerial mycelium consists of fairly long hyphae of the same thickness as the vegetative hyphae, not very much branched, without spirals, often clinging together in wisps. A differentiation into spores is never visible by direct microscopic examination. Neither is this the case in stained preparations; here the aerial hyphae break up into fragments of variable lengths, from 1.2-1.5 up to 10-12 $\mu$, showing an irregular, granulated staining.

Nutrient agar: Good growth. Vegetative mycelium raised and much wrinkled, first dirty cream-colored, later dark yellowish gray, of a soft, moist, curd-like consistency. No aerial mycelium. No pigment.

Glucose agar: Good growth. Vegetative mycelium superficial, wrinkled, honey-yellow, of a hard and cartilaginous consistency. Aerial mycelium thin, smooth, white. Yellow soluble pigment.

Potato: Good to excellent growth. Vegetative mycelium much raised and wrinkled, first cream-colored, later yellowish brown, soft and smeary. No aerial mycelium. No pigment.

Gelatin: Slow liquefaction.

Milk: Coagulation; slowly redissolved with acid reaction.

Starch: Hydrolysis.

Cellulose: No decomposition. 
Glucose broth: Rather scant growth. Granulated, yellowish sediment; no surface growth. Broth clear. No pigment. No acidity.

Nitrate: Slight or no reduction with various sources of energy.

Sucrose: Inversion.

Sucrose agar: Good growth. Vegetative mycelium superficially spreading, much raised and wrinkled, cracking, white to cream-colored, of a dry, but loose and crumbly, consistency. Aerial mycelium scant, thin, white. Faint yellow soluble pigment after 2-3 weeks.

Source: Soil.

35. Nocardia gibsonii (Erikson) comb. nov. (Erikson, D., Med. Research Council Spec. Rept. Ser. 203, 1935, 36.)

Mycelium: Young growing mycelium branches profusely at short intervals; later grows out into long, frequently wavy filaments; twisted hyphae also seen on water agar. Power of producing aerial mycelium apparently lost.

Nutrient agar: Small, cream-colored, depressed, partly confluent colonies, becoming an extensive wrinkled cream-colored skin.

Glucose agar: Cream-colored, wrinkled, membranous growth.

Potato: No growth.

Gelatin: Dull white flakes sinking as medium liquefies; liquefaction complete in 12 days.

Milk: Coagulation; some peptonization.

Starch: No hydrolysis.

Nutrient broth: Sediment of flocculi, some round and fan-shaped colonies.

Potato agar: Wrinkled, glistening, membranous growth.

Blood agar, Yellowish confluent bands, irregularly wrinkled, with small discrete colonies, clear hemolytic zone.

Dorset's egg medium: Small, round, smooth, colorless colonies with conically elevated centers.

Serum agar: Small, moist, cream-colored colonies growing into medium.

Source: The spleen in a case of acholuric jaundice. Injected into a monkey, and reisolated.

36. Nocardia fructifera (Krassilnikov) comb. nov. (Krassilnikov, N. A., Actinomycetales, Akad. Nauk. USSR, Moskau, 1941, 78.)

Vegetative hyphae: Colonies not compact, mostly of dough-like consistency, smooth or rough. Mycelium septated, hyphae breaking up into rods and in some cultures into cocci. Cells gram-positive, non-acid-fast.

Aerial mycelium: Well developed, whitish to rose-colored. Sporophores long. Straight or weakly wavy, but not spiral-shaped. Oidiospores cylindrical, elongated, 1.5 by $0.7 \mu$. 
Synthetic agar: Rose-colored to bright red and even red-orange-colored growth. Pigment not dissolved in medium.

Nutrient agar: Aerial mycelium weakly developed or absent entirely.

Gelatin: Slow liquefaction.

Milk: Coagulation and weak peptonization.

Sucrose: Inversion.

Starch: Weak hydrolysis.

Cellulose: Poor growth.

Fats: Good growth.

Source: Soil.

Remarks: One strain was obtained as a mutant of Nocardia rubra. Another strain was changed, after 8 months of cultivation, into a typical Streptomyces. This species is considered as a typical transition point between Streptomyces ruber and Nocardia rubra.

37. Nocardia africana Pijper and Pullinger. (Pijper, A., and Pullinger, B. D., J. Trop. Med. Hyg., 30, 1927, 153.)

Mycelium: Unicellular branching mycelium forming small, dense, pink colonies with short, straight, sparse, white aerial mycelium.

Nutrient agar: A few flat, pink discoid colonies.

Gelatin: Irregular pink flakes; no liquefaction.

Glucose agar: Minute, red, discrete, round colonies and piled-up, paler pink mass with thin, white aerial mycelium.

Milk: Bright red surface growth, liquid unchanged after 1 month; liquid opaque, reddish purple after 2 months; hydrolyzed, clear wine-red after 3 months.

Nutrient broth: Small pink colonies embedded in coherent, flocculent mass.

Glycerol agar: After 2 weeks, small, heaped-up, colorless masses with pink tinge around the colorless colonies, margin depressed; after 3 weeks, abundant, piled-up, pale pink growth.

Potato agar: Bright red growth, small round colonies with colorless submerged margins, and piled-up patches with stiff, sparse, white aerial mycelium.

Dorset's egg medium: Small, colorless, blister colonies, partly confluent; becoming wrinkled, depressed into medium; slight liquefaction.

Calcium agar: After 1 week, small, round, colorless colonies with red centers, margins submerged; after 2 weeks, growth bright cherry-red, confluent, with colorless margin.

Serum agar: Irregularly round, raised, wrinkled, colorless colonies; becoming dry, pink, and flaky; later piled up, brownish, friable. 
Antagonistic properties: Positive.

Source: A case of mycetoma of a foot in South Africa.

38. Nocardia pelletieri (Laveran) comb. nov. (Laveran, S., Compt. Rend. Soc. Biol., 61, 1906, 340.)

Mycelium: Composed of slender, straight, and not very long filaments forming small, dense, pink colonies with a few short, straight, isolated aerial branches.

Nutrient agar: Minute, colorless colonies and piled-up, pale pink masses.

Glucose agar: Poor growth; a few minute, pink colonies.

Potato: After 1 month, growth sparse, yellowish pink, irregularly piled up, portions with scant white aerial mycelium; after 6 months, abundant, highly piled up, small, rounded, pink masses, scant white aerial mycelium persistent.

Gelatin: Slight liquefaction; few pink flakes; later almost completely liquefied.

Milk: Soft curd; half-digested; peptonization complete in 20 days.

Nutrient broth: Small, minute, pink, clustered colonies.

Glycerol agar: Poor growth; a few moist, pink colonies.

Potato agar: Colorless blister colonies; after 3 weeks, colonies larger, showing concentric zones, submerged margins, and occasional zone or tuft of white aerial mycelium, pinkish coloration.

Blood agar: At first a few pinhead, cream-colored colonies, no hemolysis; later, colonies dense, button-shaped, with narrow, fringed margin.

Dorset's egg medium: Abundant, wrinkled, pink skin with small discrete colonies at margin in 6 days; later, surface rough, mealy; considerable liquefaction in 17 days.

Calcium agar: Colorless, small colonies; after 1 week, confluent skin, pink, buckled; medium discolored later.

Serum agar: Moist, cream-colored growth tending to be heaped up, discrete colonies at margin; becoming umbilicated.

Source: A case of crimson-grained mycetoma in Nigeria.

Remarks: Thiroux and Pelletier (Bull. Soc. Pathol. Exotique, 5, 1912, 585) considered that their cultures resembled Nocardia madurae, but they grew the organism only on Sabouraud's gelatin, on which it appeared in a constantly red, easily detachable form. Nocardia indica was regarded as identical by Pinoy, although in the original description by Laveran the organism was called Micrococcus pelletieri, because no mycelium was seen, merely coccoid bodies. Nocardia genesii Froes (Bull. Inst. Pasteur, 29, $1931,1158)$ is described as closely allied, the distinction being founded upon the fact that the red grains were smaller and much more numerous, but no cultural details are given. 
39. Nocardia maculata (Millard and Burr) Waksman and Henrici. (Millard, W., and Burr, S., Ann. Appl. Biol., 13, 1926, 580.)

Mycelium: Filamentous organisms possessing a tough, shiny colony which is cartilaginous, rarely producing an aerial mycelium, though in certain strains, it may occur frequently. Retains the mycelium form for long periods. Not acid-fast.

Gelatin: Liquefaction.

Milk: No digestion.

Starch: Hydrolysis.

Paraffin: Not utilized.

Source: Soil.

Remarks: In the young colony an orange-yellow to orange-red intercellular pigment is produced on all media, which may or may not change to black as the culture ages.

40. Nocardia rhodnii (Erikson) Waksman and Henrici. (Erikson, D., Med. Research Council Spec. Rept. Ser. 203, 1935, 37.)

Mycelium: In early stages, the minute colonies are composed of hyphal segments arranged in angular apposition; the aerial mycelium being short and straight. Later the growth becomes extensive and spreading, made up partly of long, genuinely branching filaments and partly of short segments exhibiting slipping branching, each giving rise to aerial hyphae. After 2 weeks the angular branching is very marked, delicate, spreading, herringbone patterns being formed.

Glucose agar: Abundant, coral-pink, convoluted, piled-up growth.

Gelatin: Rapid liquefaction; pale pink colonies in superficial pellicle and sediment.

Milk: Bright orange growth; medium unchanged.

Glucose broth: Thin, pink superficial pellicle, easily breaking up, and small flakes in sediment; after 2 weeks, abundant growth extending up tube.

Nutrient broth: Salmon-pink flakes in sediment and colonies on surface; after 2 weeks, abundant growth, discoloration of medium.

Czapek's agar: Minute, colorless, round colonies.

Glycerol agar: Extensive growth, dull pink colonies, round and umbilicated, becoming piled up and deeper coral; later partly submerged.

Potato agar: Abundant, pink growth, piled up, scant, stiff, white aerial mycelium at top of slant.

Dorset's egg medium: Salmon-pink, granular membrane; later piled up.

Serum agar: Extensive, reddish, confluent mass, granular, tending to be piled up; the medium around the growth shows reddish coloration in 2 weeks.

Source: From reduvid bug, Rhodnius prolixus. 
41. Nocardia gardneri (Waksman) Waksman and Henrici. (Gardner, A. D., and Chain, E., Brit. J. Exptl. Pathol., 23, 1942, 123; Waksman, in Waksman, S. A., Horning, E. S., Welsch, M., and Woodruff, H. B., Soil Sci., 54, 1942, 289.)

Mycelium: Gram-positive, branching.

Nutrient agar: Cream-colored, elevated, lichnoid growth, soft, not leathery; no aerial mycelium; very faint brownish pigment.

Glucose agar: Brownish, lichnoid growth, with wide, cream-colored edge; white to grayish aerial mycelium gradually covering surface. Reverse of growth yellowish; no soluble pigment.

Potato: Barnacle-like, brownish, spreading growth; no aerial mycelium. Medium brownish around growth.

Gelatin: Cream-colored surface ring. Rapid liquefaction. Green to greenish brown soluble pigment gradually diffuses through the liquefied portion.

Glucose-asparagine agar: Aerial mycelium develops slowly.

Tryptone broth: Growth occurs as small pellets at the base of the flask; later, a thin surface pellicle appears, which consists of a branching mycelium. Black pigment slowly produced.

Temperature: Good growth at $25^{\circ}$; slow growth at $37^{\circ} \mathrm{C}$.

Source: Isolated as an air contaminant at Oxford, England.

Remarks: On synthetic and organic media, produces an antibiotic substance (proactinomycin) which is primarily active against various grampositive bacteria. Indole not formed. No acid from glucose, lactose, maltose, mannitol, sucrose, and dulcitol.

42. Nocardia fordii (Erikson) comb. nov. (Erikson, D., Med. Research Council Spec. Rept. Ser. 203, 1935.)

Mycelium: Filaments of medium length, no spirals or markedly wavy branches. Short, straight, sparse aerial mycelium. Small oval conidia on potato agar and starch agar.

Nutrient agar: Small, creamy golden, ring-shaped colonies, and heapedup patches, becoming golden brown and convoluted.

Potato: Yellowish growth in thin line, terminal portion tending to be piled up, scant, white aerial mycelium at top of slant; after 12 days, growth abundant, golden brown, confluent, partly honeycombed, partly piled up.

Gelatin: No visible growth, slight softening in 20 days; half-liquefied after 40 days.

Milk: Coagulated, brownish surface ring.

Starch: No hydrolysis.

Nutrient broth: Few flakes at first; later abundant coherent puffball growth. 
Glycerol agar: Extensive, golden brown, convoluted, thin layer.

Blood agar: Innumerable small, yellowish, ring-shaped colonies; no hemolysis.

Dorset's egg medium: Minute, cream-colored, elevated colonies, becoming golden brown, raised, convoluted.

Calcium agar: Yellow, scale-like, closely adherent colonies; scattered white aerial mycelium.

Serum agar: Golden brown, ring-shaped and coiled, smooth colonies; no liquefaction.

Synthetic glycerol solution: Light, white, fluffy colonies, minute and in clusters.

Source: Human spleen in a case of acholuric jaundice.

43. Nocardia kuroishi Uesaka (Uesaka, I., J. Antibiotics [Japan], 5, 1952, 75-79).

Mycelium gives a weak-acid-fast reaction, but the separated cells, 0.8 by $1.3 \mu$, are non-acid-faet.

Aerial mycelium: Abundant, branching hyphae slightly curved at first, later turning around each other; cells refractive. Granules soon become visible.

Synthetic glycerol agar: Thin, pale yellow growth, covered partly with punctiform, white aerial mycelium. Yellow pigment.

Nutrient agar: Wrinkled, grayish yellow colonies. No aerial mycelium. Faint grayish brown soluble pigment.

Glucose agar: Abundant growth, at first yellowish brown then reddish brown. Scant white aerial mycelium at margin of colonies. Red to winecolored soluble pigment.

Potato: Moderate growth, at first red or brownish red, later dark brown. Aerial mycelium grayish white. Dark brown pigment.

Gelatin: Yellowish brown growth sinking into medium. No aerial mycelium. No liquefaction. Yellowish brown soluble pigment.

Milk: No coagulation. Slow liquefaction. Brown pigment.

Starch: Hydrolysis.

Glucose broth: Red colonies forming pellicle. Abundant, flocculent sediment. Dark brown, soluble pigment.

Nitrate: No reduction.

Synthetic solution: White, minute colonies on surface. Medium becomes brown.

Antagonistic properties: Produces an antibiotic.

Source: Soil.

Remarks: Lactose forms excellent source of carbon. 


\section{Description of Species of Actinomyces}

1. Actinomyces bovis Harz. (Harz, C. O., in Bollinger, O., Centrbl. Med. Wiss., 15, 1877, 485; Jahr. Münch. Thierarzeneisch., 1877, 781.)

Growth: No aerial hyphae. Radiate, sulfur-colored granules occur in the pus found in cases of actinomycosis. Large, club-shaped hyphae are seen in morbid tissues. Gram-positive. Nonmotile. Not acid-fast. Colonies smoother and softer in consistency, and more uniform than in the following species. The colonies are not adherent to the medium and growth is scantier.

Sporulation: Mycelium undergoes fragmentation very rapidly, extensive branching is rare; hyphae less than $1 \mu$ in diameter.

Semisolid media: Excellent growth, especially with paraffin seal.

Gelatin: Occasionally scant, flaky growth. No liquefaction.

Liquid media: Occasional turbidity with a light, flocculent growth.

Pigments: No soluble pigments produced on protein media. No insoluble pigments produced by growth.

Egg or serum media. No proteolytic action.

Milk: Becomes acid but usually no coagulation, no peptonization. Sometimes no growth.

Temperature: Optimum $37^{\circ} \mathrm{C}$.

Remarks: Anaerobic to microaerophilic. Bovine strains are more oxygentolerant on egg or serum media than strains of human origin belonging to the following species. Acid from glucose, sucrose, and maltose. No acid from salicin and mannitol.

2. Actinomyces israeli (Kruse) Lachner-Sandoval. (Strahlenpilze. Wolff and Israel, Arch. Pathol. Anat., 126, 1891, 11.)

Growth: Erect aerial hyphae produced in an atmosphere of reduced oxygen tension. These hyphae are occasionally septate, but no definite spores are formed. One micron or more in diameter. Large club-shaped forms are seen in morbid tissues. Gram-positive. Nonmotile. Not acid-fast.

Substrate mycelium initially unicellular and the branches may extend into the medium in long filaments or may, more or less quickly, exhibit fragmentation and characteristic angular branching. The latter resembles the phenomenon found in Corynebacterium. 
Colonies exhibit a considerable degree of polymorphism, but no stable variants have been established. Tougher in texture than those of Actinomyces bovis. Old colonies warted in appearance. Adherent to the medium. Gelatin: Occasionally scant, flaky growth. No liquefaction.

Liquid media: Usually clear.

Pigments: No soluble pigments on protein media. No insoluble pigments produced by growth.

Egg or serum media: No proteolytic action.

Milk: Becomes acid but usually does not clot. No peptonization. Frequently no growth.

Temperature: Optimum $37^{\circ} \mathrm{C}$.

Source: From 2 cases of human actinomycosis: (1) a retromaxillary tumor, (2) actinomycosis of lung and breast (Wolff and Israel).

Remarks: Anaerobic to microaerophilic. No hemolysis. 


\section{PART II}

\section{The Antibiotics}

By Selman A. Waksman and Hubert A. Lechevalier 


\section{Production of}

\section{Antibiotics by Actinomycetes}

During the last 14 years, more than 100 substances and preparations which possess antibiotic properties, i.e., have the capacity to inhibit the growth of and even to destroy various microorganisms, in dilute solutions, have been isolated from cultures of actinomycetes. Most of them have been isolated from the culture broth, and a few from the mycelium. These antibiotics vary greatly in their physical and chemical properties, their antibiotic spectra, their toxicity to animals, and their chemotherapeutic utilization. Some have very wide spectra, being active against bacteria and fungi, or gram-positive, gram-negative and acid-fast bacteria, or bacteria and larger viruses; some have somewhat narrower spectra, being active only against certain gram-positive bacteria or yeast-like fungi; still others have very narrow spectra, and are active only or largely against limited groups of organisms, such as mycobacteria or certain few viruses. Antibiotics vary in their antimicrobial action not only qualitatively but also quantitatively, one antibiotic being many times more active against a certain organism than is another antibiotic.

About 10 antibiotics produced by actinomycetes have already found application in chemotherapy, i.e., in treatment of human and animal infections. These include streptomycin and its derivative dihydrostreptomycin, chloramphenicol, chlortetracycline, viomycin, oxytetracycline, neomycin, erythromycin, and carbomycin. They are used in the treatment of a great variety of infections, caused largely by gram-positive and gramnegative bacteria, mycobacteria, rickettsiae, some of the larger viruses, some protozoa, and certain fungi. Some, such as streptomycin and cycloheximide, have found application in the treatment of plant diseases.

The isolation of these antibiotics has been a result of extensive screening programs. Large numbers of cultures of actinomycetes were isolated from soils, composts, river beds, peat bogs, and other natural substrates. They were tested systematically on different media, under different conditions of culture, and against different test organisms. Nakhimovskaia (38) and Krassilnikov and Koreniako (33) were responsible for the first screening programs, in 1937 and 1939, respectively. These studies did not yield any true antibiotic, although an enzyme-like preparation ("actinomyces lysozyme") which resulted from them may have contained or actually been an antibiotic substance (34). The same is true of another preparation obtained from a culture of an actinomyces by Gratia and Dath (24) in 1925; 
this preparation was later named by Welsch (58) actinomycetin, but this as well was not a true antibiotic and was shown by Welsch himself to contain an enzyme, which he designated as actinozyme.

The first true antibiotic from a culture of an actinomyces may, therefore, be said to have been isolated in 1940. It was designated as actinomycin (56). The first such antibiotic which possessed chemotherapeutic possibilities was isolated in 1942 (57) and was designated as streptothricin; it was a result of an extensive screening program on actinomycetes for the production of antibiotics (55). The first actinomyces-produced antibiotic that found application as a chemotherapeutic agent was streptomycin, isolated in 1943 (44).

Since then, hundreds of thousands of cultures have been isolated and tested. Systematic studies have been made of the distribution of cultures active against various groups of bacteria, fungi, and other microorganisms. Burkholder (11), for example, reported results of studies on 7,369 cultures. Of these, 1,869 inhibited the growth of Micrococcus pyogenes var. aureus, when the agar streak method was used, 261 inhibited Escherichia coli, and 514 were antagonistic to Candida albicans. According to Waksman, Horning, Welsch, and Woodruff (54) 20.1 per cent of all isolated cultures were highly antagonistic, 23.3 per cent had some activity, and 56.6 per cent were inactive upon bacteria. In general, 15 to 60 per cent of cultures of actinomycetes isolated from different natural substrates show some antimicrobial activity.

When different media are used for testing purposes, totally different results may be obtained. Uesaka (47) and others, for example, have found that certain cultures show one type of activity by the agar-streak method and another type when grown in liquid media. It must further be remembered that cultures of Streptomyces frequently produce more than one antibiotic substance. This greatly influences the problem of screening, especially when cultures of bacteria made resistant to particular antibiotics are employed or when antibiotic-producing cultures are used as test organisms, as shown by Oki and Hata (41).

Production of antibiotics is a strain characteristic rather than a species characteristic. Hence, certain strains of a given organism may produce a particular antibiotic and other strains may not. Moreover, some strains of the same organism may produce one antibiotic and others another. This is illustrated by Streptomyces griseus, of which some strains produce streptomycin, others grisein or streptocin or candicidin, and still others no antibiotic at all. The same culture may also produce more than one antibiotic, as in the case of some strains of $S$. griseus which produce streptomycin and cycloheximide, or several forms of the same antibiotic, as streptomycin and mannosidostreptomycin. 
Of the five genera of actinomycetes, the genus Streptomyces has been most productive of antibiotics, 80 or more preparations being credited so far to species of this genus. Species of Nocardia come next, with seven or eight antibiotic preparations. These are followed by species of Micromono. spora, with four or five preparations, and Thermoactinomyces, with one or two. None of the species of Actinomyces (anaerobes) have so far yielded any antibiotics. All those that have found practical application as chemotherapeutic agents are produced by species of Streptomyces.

The antibiotics produced by actinomycetes represent a great variety of chemical compounds. These compounds differ greatly in their physical properties, including solubility in water and in organic solvents; in their chemical composition; in their antimicrobial activities both in vivo and in vitro; and in their toxicity to animals. These differences are frequently not only qualitative but also quantitative in nature. Hence, the differences emphasized in these keys are often not sufficiently sharp to make such separation distinct. Frequently, a compound placed in one position in the keys could just as easily have been placed in another on the basis of certain minor differences. The difficulties involved in the classification of these antibiotics become particularly sharp when one realizes that many of them have not been crystallized. Some antibiotics have not been sufficiently described, either because of lack of information or because of certain priority questions, whereas others still represent fairly crude preparations.

The logical basis for the classification of these antibiotics is their antibiotic spectrum or range of activity against different organisms, supplemented by their physical and chemical properties. 


\section{Antibiotic Groups}

With the increasing number of antibiotics isolated from cultures of actinomycetes, whether in a crystalline state or as active crude preparations, certain broad groups become recognized. These groupings are based on biological properties, solubility and other physical properties, chemical properties, or utilization as chemotherapeutic agents. Advantage has been taken of these characteristics in the preparation of detailed keys for identification. For purposes of simplification, however, the antibiotics can be divided into twelve presently indicated groups. There is, of course, considerable overlapping among these groups, and often a particular substance may be placed with as much justification in one group as in another. There are also a number of preparations known that do not fit readily into any of these major groups but may be said to be closely related.

1. The actinomycin group. This group comprises substances which are soluble in organic solvents and to only a limited extent in water. They are active largely against gram-positive bacteria and to a much lesser extent against gram-negative bacteria and fungi. Many of them are pigmented, ranging from red and orange to yellow. Most of them are highly toxic to animals.

2. The acid-base indicator group. These substances change their color depending on reaction of substrate. They are mostly active against grampositive bacteria.

3. The streptothricin group. Members of this group are active against bacteria, including certain gram-positive and gram-negative forms, and fungi. They are basic substances, readily soluble in water and insoluble in most organic solvents. They contain amino acids. They are nonpigmented and possess a certain delayed toxicity to animals.

4. The streptomycin group. Members of this group are active against bacteria and actinomycetes but not against fungi and viruses. They are basic substances, soluble in water and insoluble in most organic solvents. They give maltol or isomaltol on hydrolysis. They are nonpigmented. They are not readily absorbed from the digestive system and possess very limited toxicity to animals.

5. The neomycin group. Active against gram-positive and gram-negative bacteria but not upon fungi or viruses. They are basic substances soluble in water and not in organic solvents. They are not readily absorbed from the digestive system. They have a toxic auditory effect when given parenterally, but are relatively nontoxic orally. They can be differentiated from the 
previous two groups by their antibiotic spectra and by the use of resistant bacterial strains.

6. The viomycin group. These are basic polypeptides, which contain guanidine or creatinine or both, and are active against the acid-fast bacteria.

7. The tetracycline-chloramphenicol group. Members of this group are active against gram-positive and gram-negative bacteria, rickettsiae, and larger viruses but not against fungi and smaller viruses. They are relatively nontoxic and can be readily absorbed from the digestive system. They are neutral or amphoteric, soluble in organic substances and to a more limited extent in water.

8. The erythromycin-carbomycin group. These are active largely against gram-positive bacteria, rickettsiae, and larger viruses, to only a limited extent against gram-negative bacteria and mycobacteria, and not against fungi and smaller viruses. They are basic compounds of limited toxicity.

9. The antifungal group active also upon bacteria and actinomycetes. They are pigmented and nonpigmented, soluble in organic solvents and to only a limited extent in water.

10. The antifungal group active only against fungi and not against bacteria and actinomycetes. Some of these substances are active primarily against yeasts and yeast-like fungi, whereas others are also active against filamentous fungi. They are soluble primarily in organic solvents; some are also soluble in water.

11. The antiprotozoan group. These substances are active selectively against various protozoa, such as spirochetes, trichomonads, trypanosomes, and amoebae. Some are also active against certain bacteria, notably cocci and other gram-positive bacteria, or against yeasts. They are acidic or basic compounds, varying in solubility and toxicity.

12. The antiviral group. These substances are active against viruses and phages and not against bacteria or fungi. They appear to be highly selective in their action. Little is known concerning their nature, mode of action, and utilization.

These 12 groups comprise most of the known antibiotics of actinomycetes. There is no doubt that with the increase of our knowledge, the isolation of more substances, the chemical purification of many of those known at present, and with greater therapeutic applications, new groups will be added and those listed here considerably modified.

These groups are believed to offer a working basis for identification of old and new compounds. The first eight groups are active primarily against bacteria and actinomycetes, but not at all or to only a limited extent (as in the case of the streptothricin group) against fungi. The ninth and tenth 
groups are active primarily against fungi, and not at all or to only a limited extent against bacteria and actinomycetes.

Groups 11 and 12 comprise the substances active primarily against protozoa and viruses, respectively. Finally, another group could comprise those substances (which we hope will be discovered) that are active primarily against neoplastic tissues and not against healthy cells of higher forms of life; these substances may be more in the nature of antibiotic-like preparations than true antibiotics, unless they are found to be also active against microorganisms.

Specific antibiotic spectra, activity against test organisms made resistant to known antibiotics, solubility properties, pigmentation, chemical nature, and toxicity to animals are among the more important properties that help to identify not only the groups but also the individual antibiotics within or outside each of these groups.

In preparing the keys for the identification of the actinomycetes, it was not the aim of the authors to make necessarily a logical classification of the antibiotics produced by actinomycetes, but to furnish workers in the field with a tool enabling them to recognize the known antibiotics and thus to facilitate establishment of any unknown agents.

It is not the absolute aim of the keys to permit final identification of a freshly isolated substance but, rather, to facilitate narrowing down the number of closely related substances. The final identification of an "unknown" antibiotic should be made only by running side to side such tests as paper chromatograms, countercurrent distribution, chemical tests, antimicrobial spectra, including use of resistant test organisms, and toxicity tests comparing the "unknown" with standard preparations of known antibiotics.

Interpretation of many of the criteria in these keys can vary from one worker to the other and with the various strains of microorganisms used in tests. Consequently, we have tried to list the same antibiotic in different groups when we thought that it could be so classified. We have not been able to cover all the possibilities and we urge the users of these keys to keep in mind the fact that an antibiotic, like a biological system, is not characterized by a single property but by a group of properties.

\section{Key I to the Identification of Antibiotics of Actinomycetes}

\section{A. Substances active primarily against gram-positive bacteria.}

I. Soluble or slightly soluble in ether and in other organic solvents, difficultly soluble in water.

1. Red, red-orange, yellow, or violet pigmented substances.

a. Red to orange, very toxic. 
$a^{1}$. Red peptide, allo-isoleucine absent, active largely against gram-positive bacteria.

\section{Actinomycin}

(Actinomycin A, B, X)

$\mathrm{b}^{1}$. Red peptide, contains D-allo-isoleucine.

Actinomycin $\mathrm{C}$

$\left(\mathrm{C}_{1}, \mathrm{C}_{2}, \mathrm{C}_{3}\right)$

$\mathrm{c}^{1}$. Closely related and incompletely described members of the actinomycin group.

Actinomycin $\mathrm{J}_{1}$

(Actinoflavin)

b. Yellowish orange bases, highly toxic.

Xanthomycin A, B

c. Saffron-yellow, weakly acidic, stable.

Resistomycin

d. Yellowish basic substances, active only against mycobacteria. Antiphlei factor

e. Reddish, acidic substance, active against mycobacteria.

Nocardamin

f. Violet pigment, soluble in water, alcohol, and ether.

Rhodocidin

g. Yellow substance, very slightly soluble in ether.

Phalamycin

2. Acid-base indicators, changing from red or yellow to blue, quinones or anthocyanins.

a. Amphoteric substituted quinones, crystalline perchlorate.

Rhodomycin A, B

b. Red pigmented, low nitrogen-containing compound.

Rhodomycetin

c. Non-nitrogen-containing quinone, alkali-soluble, ether-insoluble........Actinorhodin

d. Anthocyanin-like pigment, soluble in water.

Litmocidin

e. Reddish violet pigment.

Coelicolorin

f. Red-brown compound, yellow in acid (stable) and purple in alkali (unstable) solution.

SKCC 1377

3. Nonpigmented substances.

a. High molecular weight acids, not containing nitrogen.

$a^{1}$. Also active against fungi.

$a^{2} . \mathrm{C}_{39} \mathrm{H}_{69} \mathrm{O}_{11}$

Nigericin 
$b^{2} . \mathrm{C}_{46-47} \mathrm{H}_{80-82} \mathrm{O}_{18}$
$\mathrm{X}-206$

$c^{2} \cdot \mathrm{C}_{26} \mathrm{H}_{40} \mathrm{O}_{7}$

$\mathrm{X}-464$

$d^{2} . \mathrm{C}_{34} \mathrm{H}_{62} \mathrm{O}_{8}$

$\mathrm{X}-537 \mathrm{~A}$

$\mathrm{b}^{1}$. Also active against fungi and viruses.

Cardicin

b. Stable basic compounds, containing nitrogen.

$a^{1} . \mathrm{C}_{20} \mathrm{H}_{26} \mathrm{~N}_{2} \mathrm{O}_{6}$

b'. $\mathrm{C}_{87} \mathrm{H}_{47} \mathrm{NO}_{8}$,

Nitrosporin

$\mathrm{C}_{28} \mathrm{H}_{49} \mathrm{NO}_{8}$,

$\mathrm{C}_{24} \mathrm{H}_{41} \mathrm{NO}_{6}$,

c1. $\mathrm{C}_{25} \mathrm{H}_{43} \mathrm{NO}_{7}$

Proactinomycins A, B, C

Picromycin

$\mathrm{d}^{1}$. Other compounds.

Camphomycin

$\mathrm{e}^{1}$. Containing chlorine (?)

Exfoliatin

Sarcidin

II. Soluble in water and in organic solvents other than ether.

1. Pigmented substances.

a. Yellowish orange crystals, acid-stable.

Luteomycin

b. Yellowish green at neutral reaction.

Actinomycelin

c. Red prisms; unstable; high molecular weight.

Nocardianin

2. Nonpigmented substances.

a. Monobasic acid containing nitrogen and sulfur; primarily active against mycobacteria.

Actithiazic acid

b. Highly unstable unsaturated acid.

Mycomycin

c. Basic compounds.

$a^{1}$. Tetra acidic base containing a substituted guanidino group, active against certain bacteria and insects.

Netropsin

$\mathrm{b}^{1}$. Active against gram-positive bacteria, especially mycobacteria. .Amicetin 
d. Limited activity against mycobacteria.

Griseoflavin

e. Sulfur-containing compound.

Sulfactin

f. Yellow color changing to violet in alkaline reaction.

Vinacetin

3. Proteins or polypeptides.

a. Polypeptide; active against gram-positive rods.

Cinnamycin

b. Enzymatic in nature, lytic effect on both living and dead bacteria........Actinomycetin

(Actinozyme)

$a^{1}$. Related to actinomycetin.

Mycetin

c. Antibiotic combined with orange pigment, active against gram-positive bacteria.

Micromonosporin

d. Thermostable substance, active only against certain mycobacteria..... Antismegmatis factor

e. Active against mycobacteria.

Nocardin

f. Anti-Corynebacterium factor.

Thermomycin

B. Substances active against both gram-positive and gram-negative bacteria.

I. Basic, thermostable, nonpigmented organic compounds.

1. Soluble in water, insoluble in organic solvents, relatively complex compounds, some of which are known to contain amino sugars, not active against fungi.

a. Yielding maltol on alkaline hydrolysis, low toxicity.

Streptomycin

b. Chemically reduced streptomycin.

Dihydrostreptomycin

c. Chemical derivative of streptomycin.

Streptohydrazid

d. Enzymatic cleavage yields streptomycin and d-mannose, lower activity than streptomycin.

Mannosidostreptomycin

e. Yielding isomaltol on alkaline hydrolysis, higher toxicity than streptomycin.

Hydroxystreptomycin 
f. Other compounds related to the streptomycins.

\section{Dextromycin}

2. Soluble in water and insoluble in organic solvents; some are known to contain amino acids, amino sugars, and unidentified fragments. Active also against fungi.

a. Contain two amino acids and one amino sugar, delayed toxicity..... Streptothricin

(Streptothricin VI)

b. Compounds similar to, but not identical with, streptothricin.

Pleocidin

(Pleocidin A)

c. Other compounds similar to streptothricin.

Antibiotic 136

d. Unidentified fragments, plus an amino acid, identical with one of above.

Streptolin A, B

e. Chemically unidentified, biological activity similar to streptothricin......Actinorubin,

Lavendulin, Antibiotic $\mathrm{EI}_{5}$

f. Same as above, but greater activity against cocci.

Streptin

g. Yellow compound, more active in acid than in alkaline media.

Flaveolin

3. Compounds related to above, not active against fungi, active against bacteria resistant to streptomycin and streptothricin.

a. Active against numerous bacteria and mycobacteria.

Neomycin

(Neomycin B, Streptothricin BII)

b. Degradation products differ from those of neomycin B.

Neomycin C

(Streptothricin BI)

c. Biological or chemical degradation product of neomycin.

Neamine

(Neomycin A)

d. Substances related to the neomycins.

Catenulin

Flavomycin

Framycetin

e. Limited activity against Mycobacterium tuberculosis, low toxicity........ Roseomycin 
II. Yellow pigments, insoluble in water.

1. Soluble in organic solvents.

Thiolutin

(Aureothricin)

2. Slightly soluble in organic solvents. Unstable at alkaline pH. Griseolutein

III. Pigmented compounds.

1. Red color due to a metallic element; weak acid, containing iron and amino acids, soluble in water and phenol.

Grisein

(Antibiotic 3510)

2. Orange-yellow, contains sulfur.

Thioaurin

IV. Basic polypeptides, which contain guanidino or creatinine groups or both.

1. Both guanidino and creatinine groups present.

Vinactin A, B

2. Compound containing creatinine group but no guanidino group.

Vinactin C

3. Compound containing a guanidino but no creatinine group, fairly low toxicity.

Viomycin

4. Chemical nature not well established.

Neonocardin

C. Substances active against bacteria, rickettsiae, and larger viruses.

I. Basic substances, slowly dissolving in water.

1. Low toxicity ... Erythromycin

2. Said to possess somewhat higher toxicity than erythromycin.

Carbomycin

3. Compounds related to erythromycin and carbomycin.

Leucomycin

II. Neutral, amphoteric, or weakly basic substances, soluble in organic solvents other than ether, slightly soluble in water; yellowcolored; active against various bacteria, rickettsiae, and larger viruses.

1. Weak base, contains nonionic chlorine.

Chlortetracycline

2. Does not contain nonionic chlorine.

Oxytetracycline 
III. Nonpigmented neutral compounds.

1. Contain nonionic chlorine.

a. Active against various bacteria, low toxicity.

Chloramphenicol

D. Substances active primarily against yeasts and filamentous fungi.

I. Pigmented substances, soluble in organic solvents.

1. Insoluble in ether, slightly soluble in water; greenish yellow in color.

a. Weak base. .Fradicin

b. Weak acid

$a^{1}$. Insoluble in water.

Ascosin

Candicidin B

$\mathrm{b}^{1}$. Soluble in water

Candicidin A

2. Insoluble in water, yellowish in color, separate into ether-soluble (A) and ether-insoluble (B) fractions.

\section{Actinone}

3. Relatively insoluble in water and most organic solvents; yellow powder; found in mycelium of Streptomyces sp.

Nystatin

(Fungicidin)

4. Fairly soluble in water; highly active against Coccidioides immitis.........Antimycoin

(Fungicidin RAW)

5. Readily soluble in methanol and water containing butanol.

Chromin

6. Neutral and acid substances, active against fungi and bacteria.

Microcin A, B

II. Nonpigmented substances.

1. Insoluble in water, soluble in ether, weakly acidic phenol.

Antimycin A

2. Insoluble in water and ether; soluble in alcohols and methy] cellosolve; heat-stable; antifungal and antibacterial.

Endomycin

Helixin A, B, C and D

3. Acidic; insoluble in water and ether; unstable; antifungal and antibacterial. ..Musarin

4. Weakly acidic, containing glutarimide ring; soluble in water, chloroform and ether.

Cycloheximide 
5. Contains both basic and acidic groups.

\section{Rimocidin}

6. Insoluble in water and ether, soluble in alcohols and other organic solvents.

a. Not active against Candida albicans.

$\mathrm{a}^{1}$. Active against Trichophyton.

Phaeofacin

$b^{1}$. Not active against Trichophyton.

Rotaventin

b. Active against $C$. albicans.

Moldin

7. Insoluble in water and ether, soluble in organic solvents; free of nitrogen and sulfur; largely active against filamentous fungi.

Mycelin

8. Slightly soluble in water, soluble in organic solvents; active against filamentous fungi.

Cacaomycetin

9. Other compounds.

\section{Antiyeast factors}

E. Substances active against protozoa or insects or both.

I. Acidic substance, soluble in ether and other solvents; active against cocci and certain spirochaetes.

\section{Borrelidin}

II. Basic compounds, active against trypanosomes and gram-positive bacteria.........Puromycin

(Achromycin)

III. Soluble in water.

1. Soluble in ether, insoluble in chloroform; active against Trichomonas and gram-positive bacteria.

Streptocin

2. Insoluble in ether; active against Trichomonas and yeasts.

Trichomycin

F. Substances active primarily against viruses and phages.

I. Active against certain viruses.

1. Active against influenza B.

Ehrlichin

2. Active against eastern and western encephalomyelitis.

Abikoviromycin

II. Active against phages.

\section{Phagolessin}


G. Substances active against tumor cells.

Antitumor factors

Antitumor substance 289

Sarkomycin

\section{Key II to the Identification of Antibiotics of Actinomycetes}

A. Substances active primarily against gram-positive bacteria.

I. Active mainly against mycobacteria.

1. Active only against mycobacteria.

a. Colorless, no activity in vivo.

Actithiazic acid

b. Reddish-colored.

Nocardamin

c. Incompletely described.

Antismegmatis factor

Antiphlei factor

2. Active also against true bacteria.

a. Highly water-soluble.

Viomycin

Vinactin

Nocardin

b. Slightly water-soluble.

Amicetin

Vinacetin

Camphomycin

c. See p. 179 for erythromycin and carbomycin, p. 180 for cinnamycin, p. 180 for antibiotics X-206, X-464 and X-537A, and p. 181 for rhodocidin.

II. Strongly active also against fungi.

1. See group C for endomycin, helixin, musarin, nigericin.

2. See actinomycin p. 179 , and cardicin p. 180.

III. Strongly active also against Treponemataceae.

1. Basic, not very toxic.

2. Acidic, toxic

$$
\text { Puromycin }
$$

\section{Borrelidin}

IV. Strongly active also against gram-negative bacteria of the genus Hemophilus.

1. Containing no nitrogen.

$$
\text { Exfoliatin }
$$

2. Containing nitrogen.

a. Very toxic to mice.

Luteomycin 
b. Comparatively nontoxic to mice.

Carbomycin

Erythromycin

Leucomycin

V. Active mainly against Corynebacterium diphtheriae.

Thermomycin

VI. Active mainly against Sarcina lutea.

Sarcidin

VII. Substances not falling into the preceding groups.

1. Pigmented.

a. Yellow-green pigment at neutral reaction.

Actinomycelin

b. Yellow substances, containing no $\mathrm{N}$ or $\mathrm{S}$.

Resistomycin

c. Yellow substance containing $\mathrm{N}$ and maybe $\mathrm{S}$.

Phalamycin

d. Violet pigment.

Mycetin, Rhodocidin

e. Red pigments not acting as $\mathrm{pH}$ indicators.

$a^{1}$. Does not yield amino acids by acid hydrolysis.

Nocardianin

$b^{1}$. Yield amino acids by acid hydrolysis.

$a^{2}$. Does not contain allo-isoleucine.

Actinomycin

$\mathrm{b}^{2}$. Related substance not fully described.

Actinomycin $\mathrm{J}_{1}$

$c^{2}$. Contains allo-isoleucine.

Actinomycin $\mathrm{C}$

f. Red pigments acting as $\mathrm{pH}$ indicators. Red to yellow at acid reaction. Blue to purple at alkaline $\mathrm{pH}$.

$a^{1}$. Slightly soluble in alcohols. Light-absorption maxima at 560 and $523 \mathrm{~m} \mu$

\section{Actinorhodin}

$b^{1}$. Soluble in alcohols.

$a^{2}$. Unstable at acid and alkaline reaction. Light-absorption maxima at 575, 542, 533 and $504 \mathrm{~m} \mu$.

Rhodomycin

$\mathrm{b}^{2}$. Unstable at alkaline reaction. Insoluble in water. Light-absorption maxima at 580, 540 and $235 \mathrm{~m} \mu$. Rhodomycetin

$\mathrm{c}^{2}$. Unstable at alkaline reaction, soluble in water. SKCC 1377 
$d^{2}$. Incompletely described substances. $a^{3}$. Toxic to mice.

\section{Litmocidin}

$b^{3}$. Relatively nontoxic to mice.

2. Nonpigmented.

Coelicolorin

a. Containing sulfur.

$a^{2}$. Active against mycobacteria.

Cinnamycin

$b^{1}$. Inactive against mycobacteria.

Sulfactin

b. Containing no nitrogen and no sulfur, acidic.

Antibiotics X-206, X-464 and X-537A

c. Containing nitrogen but no sulfur.

$a^{1}$. Basic; typical light-absorption spectra.

$a^{2}$. Partly soluble in water. Peak of maximum absorption at $240 \mathrm{~m} \mu$.

Carbomycin

$b^{2}$. Insoluble in water. Peaks of maximum absorption at 250 and $320 \mathrm{~m} \mu$.

Nitrosporin

$b^{1}$. Basic; without typical light-absorption spectra.

Proactinomycin

Picromycin

3. Incompletely described.

Micromonosporin

Neonocardin

Cardicin

Griseoflavin

B. Substances active against gram-positive and gram-negative bacteria.

I. Not active against mycobacteria.

1. Active also against fungi. See group C, pp. 182-183, for thiolutin and microcins.

2. Not active against fungi.

a. Water-soluble.

\section{Grisein}

b. Soluble in organic solvents.

Xanthomycins

II. Containing no nitrogen.

1. Highly unstable unsaturated fatty acid.

Mycomycin 
See also group A, p. 178 for exfoliatin.

III. Containing nitrogen; activity against gram-negative organisms limited to members of all or one of the genera Neisseria, Hemophilus, and Brucella.

1. See group A, pp. 178-179, for luteomycin, carbomycin, erythromycin, leucomycin.

IV. Containing nitrogen and sulfur.

1. Yellow crystals, insoluble in water, neutral.

Thioaurin

Antibiotic HA-9

V. Containing nitrogen, active against rickettsiae; soluble in organic solvents.

1. White, neutral, containing nonionic chlorine.

2. Yellow.

\section{Chloramphenicol}

a. Containing nonionic chlorine.

Chlortetracycline

b. Containing no chlorine.

Oxytetracycline

Griseolutein

c. Incompletely described.

Flaveolin

VI. Containing nitrogen; not active against rickettsiae; basic; watersoluble.

1. Limited activity against gram-negative bacteria. See group A, p. 178 , for viomycin and vinactin.

2. Nearly as active against gram-negative as against gram-positive bacteria.

a. Pigmented.

$a^{1}$. Yellow compounds.

Flaveolin

$\mathrm{b}^{1}$. Red. .....Rhodocidin

b. Nonpigmented.

$a^{1}$. Activity against gram-positive and gram-negative bacteria on nutrient agar relatively limited $(3-40 \mathrm{mcg} / \mathrm{ml})$. Maximum light absorption at 236 and $296 \mathrm{~m} \mu$.

Netropsin

$b^{1}$. Inhibition of gram-negative and gram-positive bacteria on nutrient agar often requires less than $1 \mathrm{mcg} / \mathrm{ml}$. No typical ultraviolet absorption spectra.

$a^{2}$. Inactivated by cysteine. 
$a^{3}$. Streptomycin group

Streptomycin

Mannosidostreptomycin

Reticulin-Hydroxystreptomycin

Dextromycin?

$b^{2}$. Not inactivated by cysteine.

$\mathrm{a}^{3}$. Active against bacteria of the B. cereus-B. mycoides group, not active against fungi.

$a^{4}$. Neomycin group

Neomycin

Catenulin

Flavomycin

Framycetin

Dextromycin?

$\mathrm{b}^{3}$. Limited activity against bacteria of the $B$. cereus$B$. mycoides group, active against fungi.

$a^{4}$. Streptothricin group.

Streptothricin

Pleocidin

Lavendulin

Antibiotic 136

Streptolin

Streptin

Actinorubin

Antibiotic E15

Roseomycin

Remark-Many streptothricin-like substances have been described, often incompletely. We believe that the exact identification of a substance of this group requires a particularly extensive side-by-side comparison of the antimicrobial spectra and the biological properties of the various members of this group.

C. Substances active against fungi.

I. Active against both fungi and bacteria.

1. Active against gram-positive and gram-negative bacteria.

a. Neutral.

$a^{1}$. Yellow-orange; containing sulfur.

Thiolutin

$\mathrm{b}^{1}$. Reddish violet.

Microcin A

b. Acid. 
$a^{1}$. Yellowish red.

Microcin B

$b^{1}$. Highly unstable fatty acid.

c. Basic.

\section{Mycomycin}

$\mathrm{a}^{1}$. Colorless, water-soluble.

Streptothricin group

See group B, p. 182.

2. Active mainly against gram-positive bacteria.

a. Acid.

$a^{1}$. Containing no nitrogen.

Nigericin

$\mathrm{b}^{1}$. Containing nitrogen. Light absorption maximum at 240 and $267 \mathrm{~m} \mu$.

Musarin

$\mathrm{c}^{1}$. Incompletely described substances similar to musarin.

Helixin

Endomycin

b. Basic, red pigmented.

Actinomycin group

See group A, p. 179.

c. Basic, white. Camphomycin

II. Active only against fungi.

1. Rather wide antifungal spectrum; can be differentiated by their spectrum of light absorption.

a. Light-absorption maxima at 290,305 , and $316 \mathrm{~m} \mu$.

Fungicidin group

Nystatin

Antimycoin

Chromin

Rimocidin

b. Light-absorption maxima at 360, 380 and $404 \mathrm{~m} \mu$.

Ascosin

Candicidin

c. Light-absorption maxima at $240,290,335,355$, and $372 \mathrm{~m} \mu$.

Fradicin

d. Light-absorption maxima at 233 and $320 \mathrm{~m} \mu$.

Antimycin A

3. No typical light-absorption spectra or none recorded.

a. Wide antifungal spectrum.

$a^{1}$. Containing no nitrogen, insoluble in water.

Mycelin 
$\mathrm{b}^{1}$. Containing nitrogen, water-soluble.

Cycloheximide

$\mathrm{c}^{1}$. Probably containing nitrogen, insoluble in water.

Rotaventin

b. Narrow antifungal spectrum.

$\mathbf{a}^{1}$. Active against yeasts and Trichophyton.

Actinone

Phaeofacin

Moldin

$\mathrm{b}^{1}$. Active against yeasts and Trichomonas.

Trichomycin

$c^{1}$. Active mainly against $A$ spergillus niger.

Cacaomycetin

D. Substances active against true viruses and phages.

I. Active against bacterial viruses and Klebsiella pneumoniae.

Phagolessin

See also p. 180 for cardicin.

II. Active in vitro against western and eastern equine encephalomyelitis.............Abikoviromycin

III. Active in vitro against influenza $\mathrm{A}$ and $\mathrm{B}$. Active in vivo against influenza B...... Ehrlichin

E. Substances active mainly against protozoa.

I. Active against trichomonads, slightly active against bacteria, not active against yeasts.

Streptocin

II. Active against trichomonads; not active against bacteria; active against yeasts.... Trichomycin

Remark-Other compounds having marked antifungal or antibacterial activity also have antiprotozoal activity, such as thiolutin, chlortetracycline, oxytetracycline.

\section{APPENDIX}

Substances having limited activity against living bacteria.

Lytic substance dissolving dead bacteria. 


\section{Description of Antibiotics of Actinomycetes}

\section{Abikoviromycin}

Produced by: Streptomyces rubescens and S. abikoensum.

Method of extraction: Extraction of broth ( $\mathrm{pH} 7.0$ ) with ethyl acetate; concentration in vacuo. Dilution of ethyl acetate concentrate with petroleum ether and passage through a column of alumina. The elution is carried out with a mixture of petroleum ether and ethyl acetate.

Chemical and physical properties: Probably contains a carbohydrate residue, but it is not a long molecule. Very labile, especially in presence of oxygen. In aqueous solution the substance decomposes if kept 5 minutes at $100^{\circ} \mathrm{C}$, and a red pigment is formed. The intensity of the color is proportional to the antiviral activity of the preparation.

Biological activity: Active in vitro against western and eastern equine encephalomyelitis but not against Venezuela equine encephalomyelitis and Japanese encephalitis virus. Very weak activity against bacteria and fungi.

Toxicity: $\mathrm{LD}_{50}$ (mice), intravenous, $8.3 \mathrm{mg} / \mathrm{kg}$; and subcutaneous, 83 $\mathrm{mg} / \mathrm{kg}$.

Utilization: None.

Reference: Umezawa, H., Tazaki, T., and Fukuyama, S., Japan. Med. J., 4, 1951, 331-346; J. Antibiotics (Japan), 5, 1952, 469-476.

\section{Actinomycelin}

Produced by: Culture related to Streptomyces antibioticus.

Chemical and physical properties: Yellowish green pigment at neutral reaction. Soluble in water, in ethanol with intense fluorescence, and in methyl acetate; less soluble in acetone and chloroform. Most stable at neutral reaction, less so at acid and alkaline $\mathrm{pH}$.

Biological activity: Active against gram-positive bacteria, not against mycobacteria or fungi.

Toxicity: $\mathrm{LD}_{0}$ (rats), subcutaneous, $25 \mathrm{mg} / \mathrm{kg}$.

Reference: Cercos, A. P., Publ. Tech., Inst. Fitotecnia, Buenos Aires, 16, 1948, 147-156.

\section{Actinomycetin}

Produced by: Streptomyces albus.

Method of extraction: Repeated precipitation from aqueous solutions with 
ammonium sulfate followed by precipitation from concentrated aqueous solutions with acetone or alcohol. A lipoid antibacterial substance is extracted with ether from the ammonium sulfate precipitate.

Chemical and physical properties: Composed of a bacteriolytic enzyme, actinozyme, and of a lipoid antibacterial substance. Stable between $\mathrm{pH} 5.0$ and 9.0 and destroyed by ultraviolet light under $300 \mathrm{~m} \mu$. Composed of particles about $4 \mathrm{~m} \mu$.

Biological activity: Lyses dead cells of gram-positive or gram-negative bacteria. Dead gram-positive bacteria are less sensitive. Certain living gram-positive bacteria can also be lysed.

Toxicity: No data.

Utilization: Has been used in treatment of Staphylococcus infections.

Reference: Welsch, M., Phénomènes d'antibiose chez les actinomycètes.

Duculot (ed) 1947; Gratia, A., Bull. Mem. Acad. Chir., 56, 1930, 344-348; Gratia, A., and Alexander, J., Compt. Rend. Soc. Biol., 91, 1924, 1442-1443.

\section{Actinomycin}

Produced by: Streptomyces antibioticus, S. parvus, and other species of Streptomyces.

Synonyms: Actinomycin A (Waksman and Woodruff); Actinomycin B (Lehr and Berger); Actinomycin $\mathrm{X}\left(\mathrm{X}_{1}, \mathrm{X}_{2}\right)$ (Linge).

Method of extraction: Extracted from the medium with organic solvents (ether, ethyl acetate, or butanol). Crystallizes from ethyl acetate or acetone-ether mixtures as red platelets.

Chemical and physical properties: Weakly basic, red pigmented substance. Soluble in chloroform, acetone, ethanol, and ethyl acetate. Slightly soluble in water. Stable in aqueous alcohol on boiling. Unstable at acid and alkaline pH. Maximum absorption of light at $230-250 \mathrm{~m} \mu$ and $450 \mathrm{~m} \mu$ in ethanol. m.p. $250^{\circ} \mathrm{C}$. Composed of a quinonic chromophore and a peptide formed of five amino acids: threonine, proline, valine, $\mathrm{N}$-methyl valine, and sarcosine. Actinomycin $\mathrm{X}$ can be fractionated into at least two closely related compounds, $\mathrm{X}_{1}$ and $\mathrm{X}_{2}$, which differ in the ratio of the five amino acids that they contain. $\mathrm{C}_{61} \mathrm{H}_{88} \mathrm{~N}_{12} \mathrm{O}_{16}$.

Biological activity: Highly active against gram-positive bacteria and certain fungi.

Toxicity: Highly toxic, $100 \mathrm{mcg} / 24 \mathrm{gm}$ mouse causing death in 24 to 48 hours.

Reference: Waksman, S. A., and Woodruff, H. B., Proc. Soc. Exptl. Biol. Med., 45, 1940, 609-614; J. Bacterol., 42, 1941, 231-249; Waksman, S. A., and Tishler, M., J. Biol. Chem. 142, 1942, 519-528; Lehr, H., 
and Berger, J., Arch Biochem., 23, 1949, 503-505; Palgliesh, C. E., Johnson, A. W., Todd, A. R., and Vining, L. C., J. Chem. Soc., 1950, 2946-2952 ; 1952, 2672-2679; Linge, H., Thesis, Gottingen, 1951; Brockmann, H., Bohnsack, G., and Grone, H., Naturwiss., 40, 1953, 223-224.

\section{Actinomycin $\mathrm{C}\left(\mathrm{C}_{1} \mathrm{C}_{2}, \mathrm{C}_{3}\right)$}

Produced by: Streptomyces chrysomallus.

Method of extraction: Extraction of dry mycelium with benzene. Extraction of culture filtrate with butyl acetate and concentration in vacuo. Butyl acetate residue dissolved in the benzene mycelium extract and chromatographed over aluminum oxide. Elution with ethyl acetate and upon addition of carbon disulfide actinomycin $\mathrm{C}$ crystallized.

Chemical and physical properties: Red substance. Very soluble in benzene, chloroform, and acetone. Soluble in alcohol. Slightly soluble in water. m.p. $252-254^{\circ} \mathrm{C}$ with decomposition. Composed of a polypeptide containing threonine, proline, valine, $\mathrm{N}$-methyl valine, sarcosine, and d-alloisoleucine and a quinonic chromophore. By countercurrent distribution, actinomycin $\mathrm{C}$ has been shown to be not a single chemical entity. It is formed of three components, actinomycin $\mathrm{C}_{1}$ which does not contain allo-isoleucine, and actinomycin $\mathrm{C}_{2}$ and $\mathrm{C}_{3}$, which contain this amino acid. Actinomycin $\mathrm{C}_{3}$ does not contain valine. Tentative formula: $\mathrm{C}_{60-62} \mathrm{H}_{83-89} \mathrm{~N}_{11} \mathrm{O}_{16-17}$.

Biological activity: Active largely against gram-positive bacteria; limited activity against gram-negative organisms. Possesses a cytostatic action upon malignant tumors, especially of the lymphatic system.

Toxicity: Mice die within 24 hours after given $50 \mathrm{mg} / \mathrm{kg}$ orally or $5 \mathrm{mg} / \mathrm{kg}$ intraperitoneally.

Utilization: Potential use in treatment of Hodgkin's disease.

Reference: Brockmann, H., Grabhofter, N., Naturwiss., 36, 1949, 376-377; 37, 1950, 494-496; Chem. Ber., 84, 1951, 260-284; Naturwiss., 39, 1952, 429-430; Hackman, C., Strahlentherapie, 90, 1953, 296-300.

\section{Actinomycin $\mathrm{J}_{1}$}

Produced by: Streptomyces flavus.

Synonym: Actinoflavin. Closely related to, if not identical with, actinomycin.

Method of extraction: Adsorption on clay, elution with acetone, concentration of acetone, crystallization from ether.

Chemical and physical properties: Very similar to actinomycin.

Biological activity: Same as actinomycin.

Toxicity: High.

Utilization: None. 
Reference: Umezawa, H., Hayano, S., Takeuchi, T., and Mizuhara, Y., J. Penicillin (Japan), 1, 1947, 129-133; Hirata, Y., and Nakaniski, K., J. Antibiotics (Japan), 2, 1948, 181-182; Bull. Soc. Chem. Japan, 22, 1949, 121-127.

Remarks: A preparation designated as actinomycin $J_{2}$ is a mixture of actinomycin $J_{1}$ and duodecyl ester of 5-ketostearic acid. The last compound has no antibiotic activity. Actinomycin $\mathrm{J}_{2}$ is very probably the equivalent of Waksman and Woodruff's actinomycin B. Later Waksman, Geiger, and Reynolds proposed dropping the term actinomycin B to designate this fraction, since obviously it is nothing else than a mixture of actinomycin A with inert material. They proposed calling actinomycin A, actinomycin.

Reference: Hirato, Y., and Nakaniski, K., Bull. Soc. Chem. Japan, 22, 1949, 121-127; Waksman, S. A., and Woodruff, H. B., Proc. Soc. Exptl. Biol. Med., 45, 1940, 609-614; Waksman, S. A., Geiger, W. B., and Reynolds, D. M., Proc. Natl. Acad. Sci., U. S., 32, 1946, 117-120.

\section{Actinone}

Produced by: Streptomyces sp. closely related to S. antibioticus.

Method of extraction: Adsorption on active carbon, elution with methanol, concentration in vacuo, extraction with ether and separation between two active fractions: $\mathrm{A}$, ether-soluble and B, ether-insoluble.

Chemical and physical properties: Contains 1 per cent nitrogen. Not soluble in chloroform.

Biological activity: Active against Saccharomyces and Trichophyton species. No activity against other fungi and bacteria.

Toxicity: $\mathrm{LD}_{0}$ (mice), intravenous, $1000 \mathrm{mg} / \mathrm{kg}$.

Reference. Ikeda, Y., Hirai, T., and Nishimaki, T., J. Antibiotics (Japan), 3, 1950, 724-729.

\section{Actinorhodin}

Produced by: Streptomyces sp.

Method of extraction: Extraction of the mycelium with $0.5 \mathrm{~N} \mathrm{NaOH}$; neutralization yields a red precipitate. Exhaustive extraction of the precipitate with dioxane followed by concentration of solution, from which red crystals form upon standing.

Chemical and physical properties. Small, fine, red needles. Insoluble in petroleum ether, ether, and carbon tetrachloride. Slightly soluble in alcohol, acetone, dioxane and acetic acid. Soluble in pyridine, piperidine and phenol. Blue at alkaline $\mathrm{pH}$, red at acid reaction. Quinone derivative. Maximum light absorption in dioxane at 560 and $523 \mathrm{~m} \mu$. It decomposes without melting at $270^{\circ} \mathrm{C} . \mathrm{C}_{24} \mathrm{H}_{22} \mathrm{O}_{11}$. 
Biological activity: Moderately active against S. aureus.

Toxicity: No data.

Utilization: None.

Reference: Brockmann, H., Pini, H., and Plotho, O. V., Chem. Ber., 83, 1950, 161-167.

\section{Actinorubin}

Produced by: Streptomyces sp.

Synonym: Closely related to streptothricin.

Method of extraction: Adsorbed on Decalso, eluted with $\mathrm{NaCl}$. Further purification by alumina chromatography.

Physical and chemical properties: Basic substance, thermostable, dialyzes through cellophane. Soluble in water and methanol. Insoluble in ether. Helianthate gives reddish orange needles.

Biological activity: Active against gram-positive, gram-negative, and acidfast bacteria. Some activity against fungi. Limited activity against $B$. cereus-mycoides and streptococci.

Toxicity: $\mathrm{LD}_{100}$ (mice), intraperitoneal, $68.5 \mathrm{mg} / \mathrm{kg}$.

Utilization: None.

Reference: Kelner, A., and Morton, H. E., J. Bacteriol., 5s, 1947, 695-704;

Morton, H. E., Proc. Soc. Exptl. Biol. Med., 64, 1947, 327-331;

Junowicz-Kocholaty, R., and Kocholaty, W., J. Biol. Chem., 168, 1947, 757.

\section{Actithiazic Acid}

Produced by: Streptomyces virginiae, S. lavendulae.

Synonym: Mycobacidin.

Method of extraction: Adsorption on Darco G60, elution with 95 per cent ethanol, 5 per cent methanol, concentration in vacuo at $\mathrm{pH}$ 8.0. Extraction from water concentrate at $\mathrm{pH} 3.0$ with butanol; butanol chromatographed on silica gel; elution carried out with butanol; eluate concentrated and left in the refrigerator where crystallization occurs.

Chemical and physical properties: Acid, m.p. $139^{\circ}-140^{\circ} \mathrm{C}$; insoluble in water, benzene, and chloroform; soluble in alkaline solution and in lower alcohols. $[\alpha]_{D}^{25}=-60.5^{\circ}$ in absolute ethanol. No typical ultraviolet absorption. Vitamin analogue of biotin. $\mathrm{C}_{9} \mathrm{H}_{15} \mathrm{NO}_{3} \mathrm{~S} ; 4$, thiazolidone-2caproic acid.

Biological activity: Active largely against mycobacteria $(0.02-0.5 \mathrm{mcg} / \mathrm{ml})$; limited activity against other bacteria. Development of resistance slow.

Toxicity: $\mathrm{LD}_{0}$ (mice), intravenous and subcutaneous, $1.5 \mathrm{gm} / \mathrm{kg}$. 
Utilization: None. Inactive in vivo; no chemotherapeutic effect in experimental tuberculosis in mice.

Reference: Grundy, W. E., Whitman, S. L., Rdzok, E. G., Rdzok, E. J., Haines, M. E., and Sylvester, J. C., Antibiotics \& Chemotherapy, 2, 1952, 399-408; Schenck, J. R., and DeRose, A. F., Arch. Biochem. and Biophys., 40, 1952, 263-269; Clark, R. K. Jr., and Schenck, J. R., ibid., 270-276; Hwang, K., Antibiotics \& Chemotherapy, 2, 1952, 453-459; Sobin, B. A., J. Am. Chem. Soc., 74, 1952, 2247; McLamore, W. M., Celmer, W. D., Bogert, V. V., Pennington, F. C., and Solomons, I. A., ibid., 2246; Tejera, E., et al., Antibiotics \& Chemotherapy, 2, $1952,233$.

\section{Amicetin}

Produced by: Streptomyces sp., S. fasciculatus

Method of extraction: Extraction of clarified fermentation broth with $n$-butanol at $\mathrm{pH}$ 7.5. Concentration by distillation, extraction of the residue with water, lyophilization of the aqueous solution, which can be further purified by countercurrent distribution between water and methylene chloride. Amicetin can be finally crystallized from water.

Chemical and physical properties: Crystals melt at $160-165^{\circ} \mathrm{C}$. On warming to $50-70^{\circ}$ in methanol or water suspension, they are converted to a granular high melting point crystal form of the free base with the properties: m.p. $243-244^{\circ}[\alpha]_{\mathrm{D}}^{24}+116.5^{\circ}$ (c, 0.5 in $0.1 N$ hydrochloric acid); solubility in water at $25^{\circ}, 1-2 \mathrm{mg} / \mathrm{ml}$; only slightly soluble in common organic solvents. Amicetin exhibits characteristic ultraviolet absorption: in neutral aqueous solution at the maximum at $305 \mathrm{~m} \mu$; in $0.1 N$ hydrochloric acid, at $316 \mathrm{~m} \mu$; and in $0.1 N$ sodium hydroxide, at 322 $\mathrm{m} \mu$. Titration data and analyses suggest the formula $\mathrm{C}_{29} \mathrm{H}_{44} \mathrm{~N}_{6} \mathrm{O}$, for the free base.

Biological activity: Active largely against gram-positive bacteria, especially mycobacteria.

Toxicity: The acute intravenous $\mathrm{LD}_{50}$ of amicetin as the citrate complex at $\mathrm{pH} 6$ in mice is approximately $90 \mathrm{mg} / \mathrm{kg}$; the subcutaneous $L_{50}$, $600-700 \mathrm{mg} / \mathrm{kg}$. In rats the acute intravenous $\mathrm{LD}_{50}$ is approximately $200 \mathrm{mg} / \mathrm{kg}$. Amicetin is especially toxic to guinea pigs: by the subcutaneous route it is about forty times as toxic as streptomycin, but on the other hand only about one-tenth as toxic as penicillin to this same species.

Utilization: No data.

Reference: DeBoer, C., Caron, E. L., and Hinman, J. W., J. Am. Chem. Soc., 75, 1953, 499; McCormick, M. H., and Hoehn, M. M., Antibiotics \& Chemotherapy, 2, 1953, 718-720. 


\section{Antibiotic 136}

Produced by: Streptomyces lavendulae.

Synonym: Closely related to streptothricin.

Method of extraction: Adsorption at $\mathrm{pH} 7.5$ on Folin Decalso, elution with 10 per cent $\mathrm{NH}_{4} \mathrm{Cl}$; adsorption at $\mathrm{pH} 7.4$ on Darco G-60, elution with acid methanol. Precipitation of methanol solution with acetone.

Chemical and physical properties: Basic substance, soluble in methanol and water, insoluble in acetone. Most stable at acid reaction. Not inactivated by cysteine.

Biological activity: Active against gram-positive and gram-negative bacteria, mycobacteria, and fungi. Most active at alkaline reaction. Glucose decreases activity.

Toxicity: $\mathrm{LD}_{50}$ (mice), intravenous and subcutaneous, $10 \mathrm{mg} / \mathrm{kg}$.

Utilization: None.

Reference: Bohonos, N., Emerson, R. L., Whiffen, A. J., Nash, M. P., and DeBoer, C., Arch. Biochem., 15, 1947, 215-225.

\section{Antibiotic SKCC 1377}

Produced by: Streptomyces sp.

Method of extraction: Extraction of culture filtrate with benzene. Concentration in vacuo, back extraction with $0.05 \mathrm{~N} \mathrm{HCl}$, lyophilization.

Chemical and physical properties: Red-brown powder. Soluble in water, ethanol, and acetone. Insoluble in ether or benzene. In aqueous solution, yellow at acid or neutral reaction and purple at alkaline $\mathrm{pH}$. Unstable at alkaline reaction. Can stand 10 minutes at $100^{\circ} \mathrm{C}$ at $\mathrm{pH}$ 3.5. Picrate crystals melt with decomposition at $165-168^{\circ}$. Maximum light absorption of the picrate at $245 \mathrm{~m} \mu$ and $255 \mathrm{~m} \mu$.

Biological activity: Active against gram-positive bacteria. Not active against Escherichia coli and fungi.

Toxicity: $\mathrm{LD}_{50}$ (mice), intraperitoneal, $5 \mathrm{mg} / \mathrm{kg}$.

Utilization: None.

Reference: Reilly, H. C., Bact. Proc., 1952, 26.

\section{Antibiotic $\mathrm{EI}_{5}$}

Produced by: Streptomyces sp. (closely related to S. griseus).

Synonym: Closely related to actinorubin.

Method of extraction: Adsorption on charcoal, elution with acid methanol, precipitation with acetone.

Chemical and physical properties: Stable. Soluble in water. Insoluble in ether and acetone.

Biological activity: Antimicrobial spectrum similar to actinorubin.

Toxicity: Nearly equal to actinorubin. 
Utilization: None.

Reference: Weiser, R. S., Gardner, G. M., Lefler, J. S., and St. Vincent, L., Proc. Soc. Exptl. Biol. Med., 7\%, 1949, 283-287.

\section{Antibiotic HA-9}

Produced by: Streptomyces sp. related to S. lipmanii.

Synonym: Similar to thioaurin.

Method of extraction: Addition to filtered broth of 1.25 pounds of ammonium sulfate per liter and adjustment of $\mathrm{pH}$ to 6.5 . Extraction with ethyl acetate, concentration in vacuo. Further purification by chromatography on silica gel. Development of the column with ethyl acetate. Crystallization from ethyl acetate.

Chemical and physical properties: Yellow needle-like crystals. m.p. 178$180^{\circ} \mathrm{C}$. Neutral substance. Maximum light absorption at $370 \mathrm{~m} \mu$ in methanol. $[\alpha]_{D}^{25}=0$ in glacial acetic acid. Relatively insoluble in water and chloroform. Slightly soluble in ethyl acetate. Soluble in methanol. $\mathrm{C}_{7} \mathrm{H}_{6} \mathrm{~N}_{2} \mathrm{O}_{2} \mathrm{~S}_{2}$.

Biological activity: Active against gram-positive and gram-negative bacteria. Slight activity against fungi. Not inactivated by serum.

Toxicity: $\mathrm{LD}_{50}$ (mice), subcutaneous, $20 \mathrm{mg} / \mathrm{kg}$; intravenous, $15 \mathrm{mg} / \mathrm{kg}$. Utilization: Little promise, due to toxic nature.

Reference: Eiserman, W., Minieri, P. P., Abbey, A., Charlebors, J., Monorieff-Yeates, M., and Rigler, N. E., Antibiotics \& Chemotherapy, 3 , 1953, 385-392.

\section{Antibiotic X-206}

Produced by: Streptomyces sp.

Method of extraction: Can be extracted from both the mycelium and the broth filtrate. Broth extraction: extraction with butyl acetate, concentration in vacuo, back extraction with phosphate buffer ( $\mathrm{pH} 8.9)$. Mycelium extraction: extraction with ethanol and methanol, concentration in vacuo, extraction with butyl acetate, back extraction with phosphate buffer ( $\mathrm{pH}$. 8.9). Further purification of both extracts by chromatography on alumina.

Chemical and physical properties: Colorless organic acid. m.p. $126-128^{\circ} \mathrm{C}$. $[\alpha]_{\mathrm{D}}^{29}+15.0^{\circ}$ in methanol. No characteristic absorption in the ultraviolet. Unstable at acid and alkaline reactions. Soluble in alcohols, esters, acetone, ether, and petroleum ether. Insoluble in water and alkali. $\mathrm{C}_{46-47} \mathrm{H}_{80-82} \mathrm{O}_{13}$.

Biological activity: Active in vitro against gram-positive bacteria and mycobacteria. Not active in vivo against bacterial and protozoal infections. Toxicity: $\mathrm{LD}_{50}$ (mice), subcutaneous, $11 \mathrm{mg} / \mathrm{kg}$. 
Utilization: None.

Reference: Berger, J., Rachlin, A. I., Scott, W. E., Sternback, L. H., and Goldberg, M. W., J. Am. Chem. Soc., 78, 1951, 5295-5298.

\section{Antibiotic X-464}

Produced by: Streptomyces sp.

Method of extraction: Most of the antibiotic present in the cells. Extraction for the mycelium with methanol, concentration in vacuo. Extraction of concentrate with butyl acetate, concentration in vacuo. Extraction of residue with petroleum ether, concentration in vacuo. Residual oil partitioned between aqueous methanol and petroleum ether. By successive aqueous methanol extraction most of the activity is collected in the methanol, which is concentrated in vacuo. The residue is dissolved in benzene and chromatographed over alumina.

Chemical and physical properties: Colorless organic acid. m.p. $170-172^{\circ} \mathrm{C}$ with decomposition. No characteristic absorption in the ultraviolet light. $\mathrm{C}_{25} \mathrm{H}_{40} \mathrm{O}_{7}$.

Biological activity: Active in vitro against gram-positive bacteria and mycobacteria. Not active in vivo against bacterial and protozoal infections. Toxicity: $\mathrm{LD}_{50}$ (mice), intraperitoneal, $2.5 \mathrm{mg} / \mathrm{kg}$.

Utilization: None.

Reference: Berger, J., Rachlin, A. I., Scott, W. E., Sternback, L. H., and Goldberg, M. W., J. Am. Chem. Soc., 78, 1951, 5295-5298.

\section{Antibiotic X-537A}

Produced by: Streptomyces sp.

Method of extraction: Most of the antibiotic present in the cells. Extraction of cell material with butyl alcohol, concentration in vacuo, washing with sodium carbonate, drying to solid. Extraction of this solid in a Soxhlet apparatus with petroleum ether. Concentration in vacuo, crystallization from petroleum ether.

Chemical and physical properties: Colorless organic acid. m.p. $100-109^{\circ} \mathrm{C}$. $[\alpha]_{\mathrm{D}}^{26}-7.2^{\circ}$ in methanol. Maximum light absorption at 317 and 249 $\mathrm{m} \mu$ in isopropyl alcohol. Soluble in organic solvents and insoluble in water. Maximum light absorption of the sodium salt at 308 and 245 $\mathrm{m} \mu . \mathrm{C}_{34} \mathrm{H}_{52} \mathrm{O}_{8}$.

Biological activity: Active in vitro against gram-positive bacteria and mycobacteria. No activity in vivo against bacterial and protozoal infections. Toxicity: $\mathrm{LD}_{50}$ (mice), intraperitoneal, $40 \mathrm{mg} / \mathrm{kg}$.

Utilization: None.

Reference: Berger, J., Rachlin, A. I., Scott, W. E., Sternback, L. H., and Goldberg, M. W., J. Am. Chem. Soc., 73, 1951, 5295-5298. 


\section{Antimycin A}

Produced by: Streptomyces sp.

Method of extraction: Adsorption on celite at $\mathrm{pH}$ 2.5. Elution with 0.5 per cent alcohol. Concentration in vacuo, extraction with ether.

Chemical and physical properties: Acidic nitrogenous phenol. m.p. 139$140^{\circ} \mathrm{C} ;[\alpha]_{D}^{25}+64.8^{\circ}$ in chloroform. Very soluble in ether, alcohol, acetone, and chloroform. Slightly soluble in petroleum ether, benzene, and carbon tetrachloride. Insoluble in water. Very stable at room temperature. Maximum ultraviolet light absorption at 233 and 320 $m \mu$. $\mathrm{C}_{28} \mathrm{H}_{40} \mathrm{~N}_{2} \mathrm{O}_{9}$. Mild alkaline degradation yields, among other products, antimycic acid $\mathrm{C}_{11} \mathrm{H}_{14} \mathrm{~N}_{2} \mathrm{O}_{5}$ [ $\mathrm{N}$-(3-aminosalicyloyl)L-threonine]. Biological activity: Active against many yeasts and filamentous fungi. Very little activity against bacteria. Some protective action against apple scab and tomato early blight.

Toxicity: $\mathrm{LD}_{0}$ (rats), oral, $12 \mathrm{mg} / \mathrm{kg} ; \mathrm{LD}_{100}, 30 \mathrm{mg} / \mathrm{kg}$. Toxic to certain insects and spiders.

Utilization: None so far.

Reference: Leben, C., and Keitt, G. W., Phytopathology, 38, 1948, 899;

J. Am. Chem. Soc., 71, 1949, 2436-2437; 75, 1953, 1100-1104, 36233625; Science, 112, 1950, 172-173; Arch. Biochem., 28, 1950, 281-294.

\section{Antimycoin}

Produced by: Streptomyces aureus.

Synonym: Fungicidin RAW (closely related to nystatin).

Method of extraction: Extraction of broth with butanol, concentration in vacuo; extraction of residue with ethanol and precipitation of active material with ether.

Chemical and physical properties: Insoluble in ether, chloroform, and acetone. Soluble in ethyl alcohol and water. Most stable at slightly alkaline $\mathrm{pH}$. Can stand $100^{\circ} \mathrm{C}$ for 10 minutes at $\mathrm{pH}$ 7.0. Maximum light absorption at 290,305 , and $316 \mathrm{~m} \mu$.

Biological activity: Not active against bacteria, mycobacteria, and actinomycetes. Active against fungi.

Toxicity: $\mathrm{LD}_{50}$ (mice), intraperitoneal, $204 \mathrm{mg} / \mathrm{kg}$.

Utilization: Not known.

Reference: Raubitschek, F., Acker, R. F., and Waksman, S. A., Antibiotics \& Chemotherapy, 2, 1952, 179-183.

\section{Antiphlei Factor}

Produced by: Streptomyces sp., closely related to $S$. aureus.

Method of extraction: Concentration of filtrate to dryness in vacuo, extraction with methanol and precipitation with acetone. 
Chemical and physical properties: Basic substance, heat-stable, whitish yellow powder.

Biological activity: Very little, if any, activity against gram-positive and gram-negative bacteria. Active against Mycobacterium phlei and to a lesser degree against $M$. smegmatis, $M$. tuberculosis (human type), and $M$. avium.

Toxicity: Injection of $10-20 \mathrm{mg}$ of the hydrochloride into mice intramuscularly and intravenously well tolerated.

Utilization: No data.

Reference: Ouchi, N., J. Antibiotics (Japan), s, 1950, 517-523.

\section{Antismegmatis Factor}

Produced by: Streptomyces sp. Resembles S. lavendulae.

Method of extraction: Concentration by precipitation by cold.

Chemical and physical properties: Heat-stable at $\mathrm{pH}$ 7.0.

Biological activity: Most active, at an alkaline reaction, against Mycobac-

terium smegmatis, $M$. phlei. No activity against bacteria, fungi, and a pathogenic strain of $M$. bovis.

Toxicity: No reliable data available.

Utilization: None.

Reference: Kelner, A., and Morton, H. E., Proc. Soc. Exptl. Biol. Med., 63, 1946, 227-230.

\section{Antitumor Factors}

It has been definitely established that actinomycetes produce various substances which are active against neoplastic cells. Their exact nature is still unknown. It is not even known whether they should be considered as antibiotics in a true sense. They are included here because of their selective action against different cells.

\section{Antitumor Substance 289}

Produced by: Streptomyces sp.

Synonym: Closely related to luteomycin.

Method of extraction: Extraction of the broth at $\mathrm{pH} 7.0$ with ethyl acetate or butyl acetate. Back extraction with water at $\mathrm{pH} 2.0$. This process was repeated three times. The final water solution was adjusted at $\mathrm{pH}$ 5.0 and was freeze dried.

Chemical and physical properties: Basic, orange-yellow pigment. The hydrochloride is soluble in water, methanol and acetone but the sulfate has little solubility in ethanol and acetone. It can be precipitated out of aqueous solutions as the picrate, reineckate or helianthate. Positive ferric chloride test, negative ninhydrin, Molisch and Sakaguchi tests. 
Color change from orange-yellow to purple at $\mathrm{pH}$ 7.5-9.0. Ultraviolet light absorption maxima at 215,257 and $430 \mathrm{~m} \mu$. Proposed empirical formula for hydrochloride $\mathrm{C}_{26} \mathrm{H}_{33} \mathrm{NO}_{12} \cdot \mathrm{HCl}$.

Biological properties: Active against certain gram-positive bacteria, inactive against gram-negative bacteria and fungi. Has a destructive effect on the nuclei at the mitosis stage and destroys sarcomatous cells when it is injected intraperitoneally or subcutaneously in rats inoculated with Yoshida sarcoma.

Toxicity: MLD about $10 \mathrm{mg} / \mathrm{kg}$.

Utilization: Unknown.

Reference: Umezawa, H., Takeuchi, T., Nitta, K., and Maeda, K., J. Antibiotics (Japan) A, 6, 1953, 45-51.

\section{Ascosin}

Produced by: Streptomyces canescus.

Synonym: Similar to candicidin.

Method of extraction: Ascosin can be extracted both from broth and from mycelium by solvents.

Chemical and physical properties: Crude solids yellow-brown to yelloworange. Poor solubility in petroleum ether, benzene, chloroform, acetone, water, and ether; moderately soluble in methanol, ethanol, $n$-butanol, and dioxane; very soluble in pyridine, quinoline, pyrole, and piperidine. Inactivated by strong acids and bases. Maximum stability at $\mathrm{pH} 5$ to 7.5 . At $60^{\circ} \mathrm{C}$, aqueous solutions are inactivated in $24 \mathrm{hr}$; at $28^{\circ} \mathrm{C}$, solutions become inactive in 1 week; concentrated solutions more stable than dilute ones. Ultraviolet absorption in methanol, maxima at: $234,240,282,326,340,358,376$ and $399 \mathrm{~m} \mu$; minima at 261, 293, 345, 366 and $390 \mathrm{~m} \mu$. Poor diffusibility in agar. Biological activity: No activity against bacteria and streptomycetes. Mainly active against yeasts; also against certain filamentous fungi. Fungicidal action against $S$. cerevisiae strong, but not so strong as that of candicidin on C. albicans.

Toxicity: $\mathrm{LD}_{50}$ (mice), oral, $500 \mathrm{mg} / \mathrm{kg}$; subcutaneous, $168 \mathrm{mg} / \mathrm{kg}$ (severe tissue erosion at site of injection); intraperitoneal, $8.6 \mathrm{mg} / \mathrm{kg}$; intravenous, $12.5 \mathrm{mg} / \mathrm{kg}$.

Utilization: Unknown yet.

Reference: Hickey, R. J., Corum, C. J., Hidy, P. H., Cohen, I. R., Nager, U. F. B., and Krepp, E., Antibiotics \& Chemotherapy, 2, 1952, 472483.

\section{Borrelidin}

Produced by: Streptomyces rochei.

Method of extraction: Extraction of cultures with butyl acetate at $\mathrm{pH} 7$. 
Concentration, extraction with benzene, adsorption on bentonite clay, elution with methanol.

Chemical and physical properties: Acidic substance. Maximum ultraviolet absorption in isopropanol $256 \mathrm{~m} \mu$. m.p. $145-146^{\circ} \mathrm{C} ;[\alpha]_{\mathrm{D}}^{27}=-28^{\circ}$ in ethanol. $\mathrm{C}_{28} \mathrm{H}_{43} \mathrm{NO}_{6}$.

Biological activity: Active in vivo against Borrelia. Active in vitro against

Sarcina lutea and certain micrococci. In small quantities, enhances the antisyphilitic action of crystalline penicillin $\mathrm{G}$.

Toxicity: $\mathrm{LD}_{50}$ (mice), subcutaneous, $74.7 \mathrm{mg} / \mathrm{kg}$; intravenous, $39 \mathrm{mg} / \mathrm{kg}$. Utilization: None.

References: Berger, J., Jampolsky, L. M., and Goldberg, M. W., Arch. Biochem., 22, 1949, 476-478; Buck, M., Farr, C., and Schnitzer, R. J., Trans. N. Y. Acad. Sci., 11, 1948-49, 207-214.

\section{Cacaomycetin}

Produced by: Streptomyces cacaoi.

Method of extraction: Extraction with carbon tetrachloride, chromatography on alumina, elution with ethanol.

Chemical and physical properties: Slightly soluble in water, soluble in many organic solvents. Stable in neutral and acid solutions. Very unstable at alkaline $\mathrm{pH}$.

Biological activity: Active on phycomycetes and ascomycetes. Very active against Aspergillus niger.

Toxicity: $\mathrm{LD}_{0}$ (mice), subcutaneous, about $300 \mathrm{mg} / \mathrm{kg}$.

Utilization: Probably none.

Reference: Wakaki, S., Akanabe, S., Hamada, K., and Kurihara, I., J. Antibiotics (Japan), 5, 1952, 24-29.

\section{Camphomycin}

Produced by: Streptomyces sp.

Method of extraction: Extraction from culture media with methyl acetate or ether. Repeated purification with ether, alcohol, and chloroform. Crystallization from ether solutions.

Chemical and physical properties: Basic white needle-shaped crystals in bunches. Soluble in ether, methyl acetate, amyl acetate, chloroform, benzene, and alcohols. Slightly soluble in water. Stable over a wide $\mathrm{pH}$ range and is rather thermostable. m.p. $149^{\circ} \mathrm{C}$. Contains quinonic grouping. Negative Heller, Millon, Fehling, Benedict, $\alpha$-naphthol, biuret, and ferric chloride reactions. Contains $\mathrm{C}, \mathrm{H}, \mathrm{O}, \mathrm{N}$.

Biological activity: Active against gram-positive bacteria, certain gramnegative bacteria $(E$. coli), and certain mycobacteria. It also inhibits certain yeasts (Saccharomyces octosporus). It has both lytic and micro- 
biostatic action. The microbiostatic but not the lytic action is destroyed by heat $\left(15\right.$ minutes at $\left.100^{\circ} \mathrm{C}\right)$. The antibiotic is rapidly translocated in mice.

Toxicity: Intravenous injection or oral administration in mice showed no serious damage at the level of $550 \mathrm{mg} / \mathrm{kg}$.

Utilization: Unknown.

Reference: Cercos, A. P., Rev. argentina agron., 20, 1953, 53-62.

\section{Candicidin}

Produced by: Streptomyces griseus and other Streptomyces.

Synonym: Closely related to ascosin.

Method of extraction: Adsorption on Super-Cel at $\mathrm{pH}$ 2.5. Extraction of the Super-Cel cake containing also the mycelium with butanol. Butanol concentrated in vacuo. Residue washed with petroleum ether and acetone. Residue extracted with alcohol to yield water-soluble fraction $\mathrm{A}$ and leave the insoluble fraction $\mathrm{B}$.

Chemical and physical properties: Fraction A is soluble in water, alcohol, ethylene glycol, and insoluble in acetone and petroleum ether. Fraction B is slightly soluble in butanol and ethylene glycol and insoluble in water, alcohol, acetone, and petroleum ether. Both fractions give the same Rf value by paper chromatography and have the same maximum absorption of ultraviolet light at 360-362, 380-381, 404-405 $\mathrm{m} \mu$. Very unstable at acid reaction.

Biological activity: Active mainly against yeasts and also against certain filamentous fungi. Active against certain protozoa. Strong fungicidal action against Candida albicans. Maximum activity between $\mathrm{pH} 7$ and 8. Active in mice against $C$. albicans and $B$. dermatitidis.

Toxicity: About the same for fraction $\mathrm{A}$ and $\mathrm{B}$. $\mathrm{LD}_{50}$ (mice), subcutaneous, about $200 \mathrm{mg} / \mathrm{kg}$; intraperitoneal, about $50 \mathrm{mg} / \mathrm{kg}$.

Utilization: Not known yet.

Reference: Lechevalier, H., Acker, R. F., Corke, C. T., Haenseler, C. M., and Waksman, S. A., Mycologia, 45, 1953, 155-171; Kligman, A. M., and Lewis, F. S., Proc. Soc. Exptl. Biol. Med., 82, 1953, 399-404.

\section{Carbomycin}

Produced by: Streptomyces halstedii.

Synonym: Magnamycin.

Method of extraction: Not published as yet.

Chemical and physical properties: Monobasic, the free base forming white needle-shaped crystals. Melts with decomposition at $200^{\circ} \mathrm{C}$. Maximum absorption of ultraviolet light at $240 \mathrm{~m} \mu$ in ethanol. $[\alpha]_{\mathrm{D}}^{25}=-58.6^{\circ}$ 
in chloroform. The base is partly soluble in water. Acid salts such as the sulfate and hydrochloride are water-soluble. $\mathrm{C}_{43} \mathrm{H}_{69-71} \mathrm{NO}_{16}$.

Biological activity: Active against gram-positive bacteria, rickettsiae, and large viruses; not against gram-negative bacteria, except IIemophilus and Neisseria; little activity against Mycobacteria. Development of resistance slow. No cross resistance with the common antibiotics. Growth-promoting action on chicks.

Toxicity: $\mathrm{LD}_{60}$ (mice), intravenous, $550 \mathrm{mg} / \mathrm{kg}$.

Utilization: Possible utilization in treatment of infections caused by grampositive bacteria and rickettsiae. May be used as a feed supplement. Reference: Tanner, F. W., Jr., English, A. R., Lees, T. M., and Routein, J. B., Antibiotics \& Chemotherapy, 2, 1952, 441-443; Pagano, J. F., et al., ibid., $3,1953,899-902$, 910-914.

\section{Cardicin}

Produced by: Nocardia sp.

Method of extraction: Extraction of the mycelium with methanol.

Chemical and physical properties: Organic acid; insoluble in dilute acid, ether, and acetone. Soluble in methanol, ethanol, and butanol. The sodium salt is water-soluble.

Biological activity: Inhibits the growth of several gram-positive bacteria, fungi, and bacteriophages. Active in eggs against the PR-8 and FM-1 strains of the influenza virus.

Toxicity: Relatively high.

Utilization: Probably none.

Reference: Machlowitz, R. A., Charney, J., Gibson, O., Fisher, W. P., and Roberts, W. S., Abstr. of paper 122, Meeting Am. Chem. Soc., 1952, p. $13 \mathrm{~A}$.

\section{Catenulin}

Produced by: Streptomyces sp.

Synonym: Closely related to neomycin B.

Method of extraction: Adsorption and elution from carbon. Crystallized as the helianthate or as the $\mathrm{p}\left(\mathrm{p}^{\prime}\right.$-hydroxy-phenylazo)-benzene sulfonate from hot water.

Chemical and physical properties: Analysis of eatenulin sulfate: C, 31.5, per cent, H, 6.1 per cent, $\mathrm{N}, 7.9$ per cent, $\mathrm{SO}_{4}, 28.1$ per cent; optically active. $[\alpha]_{D}^{25}=+51.9^{\circ}$ in water. No typical ultraviolet absorption spectrum. The infrared absorption is typical of a polypeptide spectrum. Stable in acid solutions. Upon prolonged acid hydrolysis, catenulin yields a substance behaving by paper chromatography like 
neamine. By paper chromatography catenulin may be distinguished from neomycin A and B and the streptomycins.

Biological activity: Active against various mycobacteria; shows cross resistance with neomycin.

Toxicity: $\mathrm{LD}_{50}$ (mice), intravenous, $125 \mathrm{mg} / \mathrm{kg}$. Neurotoxicity in cats. Utilization. Probably same as neomycin.

Reference. Davisson, J. W., Antibiotics \& Chemotherapy, 2, 1952, 460462; Szybalski, W., Bact. Proc., 1952, 40.

\section{Chloramphenicol}

Produced by: Streptomyces venezuelae, S. omiyaensis.

Synonym: Chloromycetin.

Method of extraction: Extraction of acidified culture filtrates with ethyl acetate, concentration in vacuo, extraction with ether, chromatography over aluminum oxide. Can also be synthesized chemically.

Chemical and physical properties: Neutral stable substance containing nonionic chlorine. m.p. $149.7-150.7^{\circ} \mathrm{C} ;[\alpha]_{\mathrm{D}}^{25}-25.5^{\circ}$ in ethyl acetate.

Very soluble in methanol, ethanol, butanol, propylene glycol, and acetone. Slightly soluble in water. $\mathrm{C}_{11} \mathrm{H}_{18} \mathrm{~N}_{2} \mathrm{O}_{5} \mathrm{Cl}_{2}$.

Biological activity: Active against gram-positive bacteria, gram-negative bacteria, and rickettsiae.

Toxicity: $\mathrm{LD}_{50}$ (mice), intravenous, $150 \mathrm{mg} / \mathrm{kg}$.

Utilization: Treatment of infections caused by rickettsiae and bacteria. Reference: Ehrlich, J., Bartz, Q. R., Smith, R. M., Joslyn, D. A., and Burkholder, P. R., Science, 106, 1947, 417.

\section{Chlortetracycline}

Produced by: Streptomyces aureofaciens.

Synonyms: Duomycin, aureomycin.

Method of extraction: No data.

Chemical and physical properties: Weak base containing nonionic chlorine.

Very closely related to oxytetracycline. m.p. $168-169^{\circ} \mathrm{C} ;[\alpha]_{\mathrm{D}}^{23}-275^{\circ}$ in methanol. Very soluble in cellosolve, dioxane, and alkaline water. Slightly soluble in methanol, ethanol, butanol, acetone, ethyl acetate, benzene, and water. Insoluble in ether and petroleum ether. $\mathrm{C}_{22} \mathrm{H}_{23} \mathrm{~N}_{2} \mathrm{O}_{8} \mathrm{Cl}$.

Biological activity: Active against gram-positive bacteria, gram-negative bacteria, and rickettsiae. Growth-promoting action in pigs and chicks. Toxicity: $\mathrm{LD}_{50}$ (mice), intravenous, $102-134 \mathrm{mg} / \mathrm{kg}$; subcutaneous, $<600$ $\mathrm{mg} / \mathrm{kg}$; oral, $<3000 \mathrm{mg} / \mathrm{kg}$.

Utilization: Treatment of bacterial and rickettsial infections. Feed supplements. 
Reference: Aureomycin, a new antibiotic. Ann. N. Y. Acad. Sci., 51 (2), 1948, 175-342; Stephens, C. R., et al., J. Am. Chem. Soc., 74, 1952, $4976-4977$.

\section{Chromin}

Produced by: Organism related to Streptomyces antibioticus.

Method of extraction: Broth acidified to $\mathrm{pH} \mathrm{6-7}$ and treated with butanol.

Upon addition of ether to the concentrated butanol extract, a yellowish brown precipitate is formed.

Chemical and physical properties: Soluble in methanol and in water containing butanol. Readily soluble in acetone and chloroform. Labile in acid and alkali solutions. Maximum light absorption at 289, 303, and $317 \mathrm{~m} \mu$.

Biological properties: Active against a great number of yeasts and filamentous fungi, but not against bacteria.

Toxicity: Variable.

Utilization: Somewhat promising against diseases caused by Candida albicans.

Reference: Wakaki, S., Akanabe, S., Hamada, K., and Asahina, T., J. Antibiotics (Japan), 5, 1952, 677-681.

\section{Cinnamycin}

Produced by: Streptomyces cinnamoneus.

Method of extraction: The filtered broth passed through a column of IRC 50 , antibiotic eluted with $\mathrm{HCl}$, and neutralized with IR4B. Solid dissolved in 80 per cent methyl alcohol, and chromatographed over alumina.

Chemical and physical properties: Basic polypeptide, soluble in hydrated alcohols and glacial acetic acid; insoluble in ether. Maximum light absorption at $230 \mathrm{~m} \mu$ with a shoulder at $250-260 \mathrm{~m} \mu$. Aqueous solutions levorotatory. Stable from $\mathrm{pH} 2$ to $\mathrm{pH} 9$. At $92^{\circ} \mathrm{C}$ for $1 / 2$ hour no loss of activity at $\mathrm{pH} 9.0$. Contains $\mathrm{N}$ and $\mathrm{S}$; no halogen. Paper chromatography of acid and basic hydrolyzates showed presence of eight amino acids, including lanthionine and the amino acid $\mathrm{C}_{7} \mathrm{H}_{14} \mathrm{~N}_{2} \mathrm{O}_{2} \mathrm{~S}$ isolated from subtilin.

Biological activity: Active upon gram-positive rods and mycobacteria (5-55 $\mathrm{mcg} / \mathrm{ml})$; very active on Clostridium botulinum $(0.085 \mathrm{mcg} / \mathrm{ml})$; no activity against gram-positive cocci, gram-negative bacteria, and yeasts.

Toxicity. Unknown.

Utilization: Probably none.

Reference: Benedict, R. G., Dvonch, W., Shotwell, O. L., Pridham, T. G., and Lindenfelser, L. A., Antibiotics \& Chemotherapy, 2, 1952, 591-594. 


\section{Coelicolorin}

Produced by: Streptomyces coelicolor.

Method of extraction: Extraction of solid cultures with acetone at acid $\mathrm{pH}$.

Precipitation of active substance from acetone solution with water; extraction with benzene. Further purification by chromatography on alumina.

Chemical and physical properties: Indicator: red up to $\mathrm{pH} 5$, violet at $\mathrm{pH}$ $6-7$, and blue higher than $\mathrm{pH} 8$. Soluble in water at alkaline reaction. Very soluble in acetone, ethyl acetate, and chloroform. Soluble in alcohol, methanol, benzene, and ether. Insoluble in petroleum ether. m.p. $142-146^{\circ} \mathrm{C}$.

Biological activity: Active mainly against gram-positive bacteria. Toxicity: $\mathrm{LD}_{50}$ (mice), intraperitoneal, about $500 \mathrm{mg} / \mathrm{kg}$.

Utilization: Probably none.

Rejerence: Hatsuta, Y., J. Antibiotics (Japan), 2, 1949, 276-277.

\section{Cycloheximide}

Produced by: Strains of Streptomyces griseus.

Synonym: Actidione.

Method of extraction: Extraction of broth with chloroform.

Chemical and physical properties: Recrystallized from water as colorless plates; m.p. $119.5-121.0^{\circ} \mathrm{C}$; soluble in water, chloroform, and ether; thermostable, $[\alpha]_{\mathrm{D}}^{25}-3^{\circ}$ in methanol. $\mathrm{C}_{15} \mathrm{H}_{23} \mathrm{NO}_{4}$.

Biological activity: Active against yeasts and certain filamentous fungi and smuts; yeasts tend to develop rapid resistance; no activity against Candida albicans.

Toxicity: $\mathrm{LD}_{50}$ (mice), intravenous, $150 \mathrm{mg} / \mathrm{kg}$; (rats), intravenous, 2.5 $\mathrm{mg} / \mathrm{kg}$.

Utilization: Treatment of certain fungus diseases of plants.

Reference: Whiffen, A. J., Bohonos, N., and Emerson, R. L., J. Bacteriol., 52, 1946, 610-611; 56, 1948, 283-291.

\section{Dextromycin}

Produced by: Streptomyces sp.

Method of extraction: Same as streptomycin and streptothricin.

Chemical and physical properties: Basic, stable substance, water-soluble, insoluble in organic solvents, $[\alpha]_{\mathrm{D}}^{25 \prime}=+61.0 ; \mathrm{C}, 30.9$ per cent; $\mathrm{H}$, 7.2 per cent; N, 8.1 per cent. Slowly diffusible. Maltol reaction negative.

Biological activity: Same as streptomycin.

Toxicity: $\mathrm{LD}_{50}$ (mice), intravenous, $50 \mathrm{mg} / \mathrm{kg}$; subcutaneous, $750-1,000$ $\mathrm{mg} / \mathrm{kg}$. No delayed toxicity in mice. 
Utilization: Unknown.

Reference: Ogata, K., J. Antibiotics (Japan), 3, 1950, 440-444.

\section{Dihydrostreptomycin}

Produced by: Chemical derivation from streptomycin.

Production: Hydrogenation of streptomycin in aqueous solution with a platinum catalyst at atmospheric pressure. The aldehyde fraction of the streptose in the streptomycin molecule retains two atoms of hydrogen.

Chemical and physical properties: Granular white solid. m.p. $215-225^{\circ} \mathrm{C}$; $[\alpha]_{D}^{25}=-88.7^{\circ}$ in water. Not inactivated by cysteine and hydroxylamine. $\mathrm{C}_{21} \mathrm{H}_{41} \mathrm{~N}_{7} \mathrm{O}_{12}$.

Biological activity: Same as streptomycin.

Toxicity: Vestibular disturbance less than with streptomycin. Auditory impairment more common with dihydrostreptomycin than with streptomycin.

Utilization: Same as streptomycin.

Reference: Peck, R. L., Hoffhine, C. E., and Folkers, K., J. Am. Chem. Soc., 68, 1946, 1390-1391; Heck, W., Hinshaw, H. C., Lyght, C. E., and Hawkins, Z. E., Minutes 12th V. A. Conf. Chemotherapy of Tuberculosis, Atlanta, 1953, p. 125-133, 294-295.

\section{Ehrlichin}

Produced by: Streptomyces lavendulae.

Method of extraction: Culture filtrate adjusted at $\mathrm{pH} 2.0$ with concentrated $\mathrm{HCl}$. A dark brown precipitate collected by centrifugation.

Chemical and physical properties: Stable at neutrality and alkaline $\mathrm{pH}$.

Nondialyzable. Inactivated in vitro by horse serum. Unaffected by tryptic digestion.

Biological activity: Inhibitory to influenza A and influenza B in vitro. Active in vivo against influenza B. Inactive against bacteria, fungi, Chlamydozoaceae, pox viruses, and bacterial viruses.

Toxicity: $\mathrm{LD}_{0}$ (mice), intraperitoneal, $100 \mathrm{mg} / \mathrm{kg}$; subcutaneous, $300 \mathrm{mg} / \mathrm{kg}$. Utilization: None.

Reference: Groupé, V., Frankel, J. W., Lechevalier, M. P., and Waksman, S. A., J. Immunol., 67, 1951, 471-482.

\section{Endomycin}

Produced by: Streptomyces sp., related to S. albus.

Synonym: Closely related to helixin.

Method of extraction: Antibiotic present largely in mycelium. Extraction with butanol, concentration to a gum, extraction with ether, evapora- 
tion to dryness; suspension of solid in water, extraction with ether to remove impurities; adjustment of $\mathrm{pH}$ of water portion to 4.5 with formation of an active precipitate.

Chemical and physical properties: High molecular weight acid containing 3.7 per cent N. Soluble in alcohols and methyl cellosolve. Insoluble in water, ether, chloroform, benzene, ethyl acetate, and other fat solvents. Stable at $100^{\circ} \mathrm{C}$ for 30 minutes.

Biological activity. Active against bacteria and fungi. More active against yeast-like fungi $(0.5-2.5 \mathrm{mcg} / \mathrm{ml})$ than against filamentous fungi $(12-25 \mathrm{mcg} / \mathrm{ml})$. More active against gram-positive bacteria than against gram-negative bacteria.

Toxicity: No clear data available. Mice killed by injections of $1 \mathrm{gm} / \mathrm{kg}$, not by $0.5 \mathrm{gm} / \mathrm{kg}$.

Utilization: No information.

Reference: Gottlieb, D., Bhattacharyya, P. K., Carter, H. E., and Anderson, H. W., Phytopathology, 41, 1951, 393-400.

\section{Erythromycin}

Produced by: Streptomyces erythreus.

Synonyms: Ilotycin, Erythrocin.

Method of extraction: From the alkaline broth with benzene, ethyl acetate, carbon tetrachloride, etc.

Chemical and physical properties: Basic compound soluble in water (2 $\mathrm{mg} / \mathrm{ml}$ ), very soluble in alcohols and acetone, chloroform, and ethyl acetate. Moderately soluble in ether, ethylene dichloride, and amyl acetate. Molecular weight $725 .[\alpha]_{D}^{25}-78^{\circ}$ in alcohol. Maximum light absorption at $280 \mathrm{~m} \mu$. The acid degradation of erythromycin yields two bases, dimethyl amine and a second base having the following empirical formula $\mathrm{C}_{8} \mathrm{H}_{17} \mathrm{NO}_{3} \cdot \mathrm{HCl}$.

Biological activity: Active against gram-positive bacteria, including corynebacteria; not active against gram-negative bacteria, except Neisseria, Hemophilus, and Brucella; active against mycobacteria, rickettsiae, and large viruses; active against certain protozoa. Development of resistance slow.

Toxicity: $\mathrm{LD}_{\mathrm{s} 0}$ (mice), oral, about $4000 \mathrm{mg} / \mathrm{kg}$; subcutaneous, $1800 \mathrm{mg} / \mathrm{kg}$. Utilization: Can be used in certain cases to replace penicillin, chlortetracycline, oxytetracycline, or chloramphenicol.

Reference: McGuire, J. M., Antibiotics \& Chemotherapy, 2, 1952, 281; Heilman, F. R., Herrell, W. E., Wellman, W. H., and Geraci, J. E., Proc. Staff Meetings, Mayo Clinic, 27, 1952, 285; Haight, T. H., and Finland, M., New Engl. J. Med., 247, 1952, 227. 


\section{Exfoliatin}

Produced by: Streptomyces exfoliatus.

Method of extraction: Broth extraction with ethyl acetate, concentration to a syrup in vacuo. The syrup is washed with petroleum ether and ether, leaving the crude exfoliatin. Can be crystallized from ethanol. Chemical and physical properties: Colorless needle crystals soluble in ethanol, acetone, chloroform, and ethyl acetate. Very slightly soluble in petroleum ether, ether, and water; m.p. $172^{\circ} \mathrm{C} . \mathrm{C}_{27} \mathrm{H}_{40} \mathrm{O}_{16} \mathrm{Cl} \cdot \mathrm{H}_{2} \mathrm{O}$.

Biological activity: Active against gram-positive bacteria. Almost inactive against gram-negative bacteria, except members of the genus Hemophilus.

Toxicity: $\mathrm{LD}_{60}$ (mice), subcutaneous, about $500 \mathrm{mg} / \mathrm{kg}$.

Utilization: Not known yet.

Reference: Umezawa, H., Takahashi, S., Takeuchi, T., Maeda, K., and Okami, Y., J. Antibiotics (Japan), 5, 1952, 466; Japan. J. Med. Sci. Biol., 5, 1952, 311-316.

\section{Flaveolin}

Produced by: Streptomyces sp. Resembles S. flaveolus.

Method of extraction: Adsorption from culture filtrate at neutrality on charcoal. Elution with acidic acetone. Concentration in vacuo. Extraction at $\mathrm{pH} 8.0$ with butanol. Back extraction of the butanol with acidic water. Further purification by countercurrent distribution between butanol and phosphate buffer at pH 5.5.

Chemical and physical properties: Yellow substance, basic. The hydrochloride is soluble in water, methanol, and ethanol. Slightly soluble in acetone, benzene, butanol, and chloroform. Insoluble in ether, petroleum ether, and ethyl acetate. Millon, Biuret, and Sakaguchi reactions negative. Maximum stability at acid reaction ( $\mathrm{pH} 2.0$ ).

Biological activity: Active against gram-positive bacteria, gram-negative bacteria, mycobacteria, and fungi. More active in acidic test media than in alkaline media.

Toxicity: $\mathrm{LD}_{50}$ (mice), intravenous, about $30 \mathrm{mg} / \mathrm{kg}$.

Utilization: No data.

Reference: Takahashi, B., J. Antibiotics (Japan), 6, 1953, 11-20.

\section{Flavomycin}

Produced by: Streptomyces roseoflavus.

Synonym: Neomycin.

Method of extraction: Same as streptothricin and streptomycin. 
Chemical and physical properties: Organic base, water-soluble and insoluble in organic solvents. Stable.

Biological activity: Active against gram-positive, gram-negative, and acidfast bacteria.

Toxicity: $\mathrm{LD}_{0}$ (mice), intravenous, about $200 \mathrm{mg} / \mathrm{kg}$; subcutaneous, about $500 \mathrm{mg} / \mathrm{kg}$.

Utilization: Probably same as neomycin.

References: Aiso, K., Miyaki, K., Yanagizawa, F., Arai, T., and Hayaski, M., J. Antibiotics (Japan), 3, 1950, 87-92; Arai, T., ibid., 4, 1951, 215-220.

\section{Fradicin}

Produced by: Streptomyces fradiae.

Method of extraction: Extraction of the broth with butanol, concentration of butanol in vacuo, extraction with ethanol, concentration, precipitation of an oil with acetone and petroleum ether. The oil is dissolved in $t$-butanol and lyophilized.

Chemical and physical properties. Weak base soluble in alcohols, especially in propylene glycol and chloroform. Insoluble in ether, carbon tetrachloride, benzene, acetone, and water. Stable at neutrality. Maximum light absorption at $240,290,335,355$ and $372 \mathrm{~m} \mu$. Tentative empirical formula $\mathrm{C}_{30} \mathrm{H}_{34} \mathrm{~N}_{4} \mathrm{O}_{4}$.

Biological activity: Not active against bacteria. Active against most fungi, mainly at alkaline $\mathrm{pH}$.

Toxicity: $\mathrm{LD}_{50}$ (mice), intraperitoneal and oral, $4 \mathrm{mg} / \mathrm{kg}$. Very irritating to rabbit skin.

Utilization: Most likely none.

Reference: Swart, E. A., Romano, A. H., and Waksman, S. A. Proc. Soc. Exptl. Biol. Med., 73, 1950, 376-378; Hickey, R. J., and Hidy, P. H., Science, 11s, 1951, 361-362.

\section{Framycetin}

Produced by: Streptomyces sp.

Synonym: Soframycine, antibiotic 185.

Method of extraction: Adsorption on activated charcoal and elution with acid alcohol. Can also be extracted by adsorption on cation exchange resins and elution with an acid solution.

Chemical and physical properties: Basic, nonpigmented substance, water soluble and insoluble in organic solvents. Chemical analysis of the sulfate: $\mathrm{C}=33$ per cent, $\mathrm{H}=5.3$ per cent, $\mathrm{N}=9.1$ per cent, $\mathrm{O}=23.5$ per cent, $\mathrm{SO}_{4}=29$ per cent. $[\alpha]_{\mathrm{D}}^{18}=+50^{\circ}$ in water concentration 1 per cent. All the nitrogen in the molecule is in the form of amino 
groups, but it is not a polypeptide. Positive ninhydrine reaction.

No guanidine groups. No typical ultraviolet absorption spectrum. The picrate, picrolonate and reineckate can be crystallized.

Biological activity: Active against gram-positive, gram-negative bacteria and mycobacteria. Limited activity against fungi.

Toxicity: $\mathrm{LD}_{50}$ (mice), intravenous, $65 \mathrm{mg} / \mathrm{kg}$.

Utilization: Topical application for bacterial infections. Specially active in staphylococcic infections. Can be used systemically when other less toxic antibiotics are not active.

Reference: Decaris, L. J., Ann. Pharm. France, 9, 1953, 44; Tremblay, E. C., Destouches, P., and Karatchenzeff, N., Presse Med., 61, 1952, Feb. 28; Lutz, C. R., Compt. Rend. Acad. Sci., 236, 1953, 157; Hagemann, G. R., Thesis, Univ. Paris, 1952.

\section{Grisein}

Produced by: Streptomyces griseus.

Method of extraction: Adsorption on activated charcoal and elution with 95 per cent ethanol followed by precipitation with methanol and acetone. Chemical and physical properties: Soluble in water, slightly soluble in 95 per cent ethanol, insoluble in ethanol, ether, and acetone. Stable. Not inactivated by cysteine. Inactivated by iron. $\mathrm{C}_{40} \mathrm{H}_{61} \mathrm{~N}_{10} \mathrm{O}_{20} \mathrm{SFe}$.

Biological activity: Active against Escherichia coli, Micrococcus pyogenes var. aureus, Salmonellae and Shigellae. No activity against Pseudomonas, Proteus, and Mycobacterium. Development of resistance rapid. Toxicity: No reliable data available.

Utilization: None.

Reference: Reynolds, D. M., and Waksman, S. A., J. Bacteriol., 55, 1948, 739-752; Garson, W., and Waksman, S. A., Proc. Natl. Acad. Sci. U.S., $34,1948,232-239$.

\section{Griseoflavin}

Produced by: Streptomyces griseoflavus.

Method of extraction: Adsorption on acid clay at $\mathrm{pH} 2.0$, elution with 80 per cent acetone at $\mathrm{pH}$ 7.0. Evaporation of acetone, aqueous solution extracted with ethyl acetate, evaporation to dryness. Brown powder dissolved in methanol, chromatography over aluminum oxide. Crystallization from ethanol.

Chemical and physical properties: Colorless crystals, m.p. $210-215^{\circ} \mathrm{C}$ (decomposition). Soluble in methanol, ethanol, propanol, phenol, acetic acid and alkaline water. Slightly soluble in water, ethyl acetate and butyl acetate. Insoluble in ether, petroleum ether, benzene and chloroform. Negative biuret, ninhydrin, Sakaguchi, Molisch and $\mathrm{FeCl}_{3}$ tests. 
Biological properties: Active primarily against gram-positive bacteria, limited activity against gram-negative bacteria and mycobacteria. Strains of Staphylococci, Vibrio comma and one Mycobacterium were the most sensitive organisms $(1-2 \mathrm{mcg} / \mathrm{ml})$.

Toxicity: $\mathrm{LD}_{50}$ (mice), intraperitoneal, more than $250 \mathrm{mg} / \mathrm{kg}$. Utilization: Unknown.

Reference: Waga, Y., J. Antibiotics (Japan), A 6, 1953, 66-72.

\section{Griseolutein}

Produced by: Streptomyces griseoluteus.

Method of extraction: Extraction at pH 2.0 with ethyl acetate. Ethyl acetate concentrate chromatographed over alumina. Yellow fractions contain the antibiotic which crystallizes at $0^{\circ} \mathrm{C}$.

Chemical and physical properties: Yellow needle-shaped crystals. $\mathrm{C}=59.5$ per cent; $\mathrm{H}=4.4$ per cent; $\mathrm{N}=8.0$ per cent. Insoluble in water and ether, slightly soluble in ethyl acetate and alcohol. Soluble but unstable at alkaline $\mathrm{pH}$. Maximum stability at acid reaction.

Biological activity: Active against gram-positive and gram-negative bacteria. Toxicity: No toxicity to mice injected subcutaneously at $1000 \mathrm{mg} / \mathrm{kg}$. Utilization: Unknown.

Reference: Umezawa, H., Hayano, S., Maeda, K., Ogata, Y., and Okami, Y., J. Antibiotics (Japan), 4, 1951, 34-40.

\section{Helixin}

Produced by: Streptomyces sp.

Synonym: Closely related to endomycin.

Method of extraction: Precipitation at $\mathrm{pH}$ 3.0, extraction of the precipitate with ethanol.

Chemical and physical properties: Soluble in ethanol, methanol, pyridine, glacial acetic acid; slightly soluble in n-butanol, acetone, chloroform; insoluble in ether, petroleum ether, benzene, ethyl acetate, and carbon tetrachloride. Most active at alkaline $\mathrm{pH}$. Somewhat less stable than endomycin.

Biological activity: Active against filamentous and nonfilamentous fungi in concentration of $15 \mathrm{mcg}$ or less per $\mathrm{ml}$. Active against bacteria in concentrations of $30 \mathrm{mcg}$ or more per $\mathrm{ml}$.

Toxicity: Inhibition of seed germination by 25 to $100 \mathrm{mcg} / \mathrm{ml}$. Toxic to tomato cuttings in concentration of $7.5-15 \mathrm{mcg} / \mathrm{ml}$.

Utilization: No data.

Reference: Leben, C., Stessel, G. J., and Keitt, G. W., Mycologia, 44, 1952, 159-169; Leben, C., and Keitt, G. W., Phytopathology, 42, 1952, 168-170. 


\section{Hydroxystreptomycin}

Produced by: Streptomyces griseocarneus., S. reticuli.

Synonym: Reticulin.

Method of extraction: Same as streptomycin.

Chemical and physical properties: Same as streptomycin. Can be differentiated from streptomycin by paper chromatography. Same chemical structure as streptomycin except for hydroxylation of the streptose molecule.

Biological activity: Same as streptomycin.

Toxicity: About the same as streptomycin and dihydrostreptomycin. Very ototoxic to cats.

Utilization: None.

Reference: Benedict, R. G., Stodola, F. H., Shotwell, O. L., Borud, A. M., and Lindenfelser, L. A., Science, 112, 1950, 77-78; Grundy, W. E., Whitman, A. L., Hanes, M. E., and Sylvester, G. C., Antibiotics \& Chemotherapy, 1, 1951, 309-317; Ambrose, A. M., Proc. Soc. Exptl. Biol. Med., 76, 1951, 466; Hawkins, G. E., Trans. 10th Conf. Chemotherapy of Tuberculosis, Atlanta, 1951, 224-227; Hosoya, S., Soeda, M., Komatsu, N., Sonoda, Y., Japan. J. Exptl. Med., 20, 1949, 327335 .

\section{Lavendulin}

Produced by: Streptomyces lavendulae.

Synonym: Closely related to streptothricin.

Method of extraction: Adsorbed on Decalso, eluted with sodium chloride.

Further purification by alumina chromatography.

Chemical and physical properties: Basic substance, thermostable, dialyzes through cellophane. Soluble in water and methanol; insoluble in ether. Biological activity: Active against gram-positive, gram-negative, and acidfast bacteria. Some activity against fungi. Limited activity against

$B$. cereus-mycoides and streptococci.

Toxicity: $\mathrm{LD}_{100}$ (mice), intraperitoneal, $25 \mathrm{mg} / \mathrm{kg}$.

Utilization: None.

Reference: Kelner, A., and Morton, H. E., J. Bacteriol., 58, 1947, 695-704;

Morton, H. E., Proc. Soc. Exptl. Biol. Med., 64, 1947, 327-331; Junowicz-Kocholaty, R., and Kocholaty, W., J. Biol. Chem., 168, 1947, 757.

\section{Leucomycin}

Produced by: Streptomyces kitasatoensis.

Synonym: Closely related to erythromycin.

Method of extraction: Extraction of broth at $\mathrm{pH} 7.0$ with benzene or ethyl acetate. 
Chemical and physical properties: Basic compound. White column-shaped crystals. Free base slightly soluble in water and very soluble in alcohols, acetone, ethyl acetate, butyl acetate, chloroform, and benzene; insoluble in petroleum ether. m.p. $126-129^{\circ} \mathrm{C} ;[\alpha]_{D}^{25}-60.4^{\circ}$ in ethanol. Maximum light absorption at $230-232 \mathrm{~m} \mu$ in ethanol. Stable at neutrality. $\mathrm{C}_{27} \mathrm{H}_{42} \mathrm{NO}_{10}$.

Biological activity: Very active against gram-positive bacteria, including anaerobes. Very limited activity against gram-negative bacteria except Hemophilus and Neisseria. Limited activity against mycobacteria. No activity against fungi. Active in vivo against Clostridium welchii, Spirochaeta recurrentis, rickettsiae, and lymphogranuloma virus.

Toxicity: $\mathrm{LD}_{50}$ (mice), intravenous, $650 \mathrm{mg} / \mathrm{kg}$; subcutaneous, $800 \mathrm{mg} / \mathrm{kg}$. Reference: Hata, T., Sano, Y., Ohki, N., Yokoyama, Y., Matsumae, H., and Ito, S., J. Antibiotics (Japan), A 6, 1953, 87-89.

\section{Litmocidin}

Produced by: Nocardia cyanea (Proactinomyces cyaneus-antibioticus).

Method of extraction: Extraction of agar cultures with water, acidification to $\mathrm{pH} 3.5$, adsorption on charcoal. Elution with acid acetone, concentration in vacuo to dryness. Residue dissolved in ethanol and precipitated with water.

Chemical and physical properties: Pigment red under acid conditions and blue under alkaline reaction. Water soluble at alkaline reaction. Stable. m.p. 144 to $146^{\circ} \mathrm{C}$. Closely related to anthocyans.

Biological activity: Very active against gram-positive bacteria and mycobacteria, limited activity against gram-negative bacteria. No action against Micrococcus pyogenes var. aureus in mice.

Toxicity: $\mathrm{LD}_{50}$ (mice), intraperitoneal, $50 \mathrm{mg} / \mathrm{kg}$.

Utilization: None.

Reference: Gause, G. F., J. Bacteriol., 51, 1946, 649-653; Brazhnikova, M. G., ibid, 655-657.

\section{Luteomycin}

Produced by: Streptomyces tanashiensis (related to S. aureus and S. antibioticus).

Method of extraction: Extraction from broth with organic solvents (chloroform, ethyl acetate) at $\mathrm{pH}$ 7.5-8.0. Back extraction of organic solvents with water at $\mathrm{pH}$ 3.0. Chromatography on alumina column. Column developed with acetone or methanol.

Chemical and physical properties: Basic compound. Hydrochloride easily soluble in chloroform; moderately soluble in water, ethanol, methanol, acetone, ethyl acetate, and butyl acetate; and insoluble in ether, 
petroleum ether, and benzene. Maximum light absorption at 270 and $430 \mathrm{~m} \mu$. Unstable substance with maximum stability on the acid side. m.p. $199-200^{\circ} \mathrm{C}$ with decomposition. $\mathrm{C}_{23} \mathrm{H}_{29} \mathrm{NO}_{9}$.

Biological activity: Active mainly against gram-positive bacteria and mycobacteria. Very limited activity against the enterobacteriaceae but active against Hemophilus and Brucella. Development of resistance slow. Active in vivo against $B$. melitensis, $H$. pertussis, and pneumococci.

Toxicity: $\mathrm{LD}_{50}$ (mice), subcutaneous and intravenous, about $10 \mathrm{mg} / \mathrm{kg}$. Utilization: Unknown.

Reference: Hata, T., Ohki, N., and Higuchi, T., Kitasato Arch. Exp. Med., 32, 1949, 229-242; J. Antibiotics (Japan), s, 1950, 313-325; 5, 1952, 529-534; Sano, Y., ibid., 535-538.

\section{Mannosidostreptomycin}

Produced by: Streptomyces griseus.

Synonym: Streptomycin B.

Method of extraction: Mannosidostreptomycin can be separated from streptomycin by chromatography over acid-washed alumina.

Chemical and physical properties: Mannosidostreptomycin is a D-mannoside of streptomycin. m.p. $178-179^{\circ} \mathrm{C}$ with decomposition; $[\alpha]_{\mathrm{D}}^{25}=47^{\circ}$ in water. $\mathrm{C}_{27} \mathrm{H}_{49} \mathrm{O}_{17} \mathrm{~N}_{7}$.

Biological activity: Same spectrum as streptomycin but four to eight times less active than streptomycin on a weight basis. In experimental infections mannosidostreptomycin is about three times less active than streptomycin.

Toxicity: $\mathrm{LD}_{50}$ (mice), intravenous, $250 \mathrm{mg} / \mathrm{kg}$.

Utilization: None.

Reference: Fried, J., and Titus, E., J. Biol. Chem., 168, 1942, 391-392;

J. Am. Chem. Soc., 70, 1948, 3615-3618; Rake, G., McKee, C. M., Pansy, F. E., and Donovick, R., Proc. Soc. Exptl. Biol. Med., 65, 1947, 107-112.

\section{Microcin A, B}

Produced by: Micromonospora sp.

Method of extraction: Extraction from broth filtrate at $\mathrm{pH} 2.0$ with ethyl acetate. Back extraction of ethyl acetate with phosphate buffer $(\mathrm{pH}$ 7.0). Ethyl acetate fraction contains microcin A; the buffer, microcin B. Chemical and physical properties: Microcin A: neutral, reddish violet, insoluble in water. Microcin B: acid, yellowish red, slightly soluble in water.

Biological activity: Active against fungi, gram-positive and gram-negative bacteria. 
Toxicity: $\mathrm{LD}_{50}$ (mice), intravenous, $625 \mathrm{mg} / \mathrm{kg}$.

Utilization: None.

Reference: Taira, T., and Fujii, S., J. Antibiotics (Japan), 5, 1952, 185-187.

\section{Micromonosporin}

Produced by: Micromonospora sp.

Method of extraction: Precipitation by saturation with ammonium sulfate and dialyzation of the precipitates against water.

Chemical and physical properties: Protein associated with a carbohydrate. Biological activity: Active against gram-positive bacteria. Not active against gram-negative bacteria.

Toxicity: No data.

Utilization: None.

Reference: Waksman, S. A., Geiger, W. B., and Bugie, E., J. Bacteriol., $58,1947,355-357$.

\section{Miramycin}

Produced by: Streptomyces mirabilis.

Method of extraction: Unknown.

Chemical and physical properties: Heat stable.

Biological activity: Active against gram-positive and gram-negative bacteria.

Toxicity: Said to be nontoxic.

Utilization: Unknown.

Reference: Ruschmann, G., Die Pharmazie, 7, 1952, 542-550, 639-648, 823-831.

\section{Moldin}

Produced by: Streptomyces phaeochromogenus.

Method of extraction: Extraction of broth or the mycelium with ethyl acetate, concentration in vacuo to a syrup. The syrup is washed with petroleum ether and water. The residue is dissolved in ethanol and precipitated with water.

Chemical and physical properties: Soluble in ethanol and ethyl acetate. Slightly soluble in petroleum ether, ether, and benzene.

Biological activity: Active against Candida, Trichophyton, Histoplasma capsulatum, and Cryptococcus neoformans.

Toxicity: $\mathrm{LD}_{0}$ (mice), intraperitoneal, about $10 \mathrm{mg} / \mathrm{kg}$.

Utilization: None.

Reference: Maeda, K., Okami, Y., Taya, O., and Umezawa, H., J. Antibiotics (Japan), 5, 1952, 465; Japan. J. Med. Sci. Biol. 5, 1952, 327-339. 


\section{Musarin}

Produced by: Streptomyces sp.

Method of extraction: Present in broth and mycelium. Acidified to $\mathrm{pH} 4.0$ with $\mathrm{H}_{3} \mathrm{PO}_{4}$ with formation of a precipitate. The filtrate extracted with neutral phosphate buffer. Back extraction with butanol, concentration in vacuo. Residue dissolved in methanol and precipitated with ether.

Chemical and physical properties: Acidic substance. Soluble in aqueous acetone and alcohol; insoluble in water or acetone. Sodium salt soluble in water and alcohols; insoluble in ether and acetone. Fairly stable in the sodium form. Maximum light absorption at 240 and $267 \mathrm{~m} \mu$. $\mathrm{C}_{36} \mathrm{H}_{60} \mathrm{O}_{14} \mathrm{~N}_{2}$.

Biological activity: Active against filamentous fungi, gram-positive bacteria, and mycobacteria.

Toxicity: No data.

Utilization: None to date.

Reference: Arnstein, H. R. V., Cook, A. H., and Lacey, M. S., J. Gen. Microbiol., 2, 1948, 111-122.

\section{Mycelin}

Produced by: Streptomyces roseoflavus.

Method of extraction: Extraction of the mycelium with methanol. Concentration in vacuo. Precipitation of inactive materials with ethanol and barium hydroxide leaves crude mycelin in solution. Further purification by chromatography on alumina.

Chemical and physical properties: Mycelin crystallizes from acetone in form of prisms. Soluble in chloroform, butanol, methanol, ethanol, acetone, and benzene. Insoluble in water, ether, and petroleum ether. Contains no sulfur or nitrogen. Stable.

Biological activity: Not active against bacteria. Active against filamentous fungi.

Toxicity: No data.

Utilization: No data.

Reference: Aiso, K., Arai, T., Washida, K., and Tanaami, T., J. Antibiotics (Japan), 5, 1952, 217-219.

\section{Mycetin}

Produced by: Streptomyces violaceus.

Method of extraction: Extraction of dried, pulverized agar culture with a mixture of equal parts of ethanol and dichloroethane (the latter can 
be replaced by chloroform or benzene). The solvent is evaporated and substance dissolved in ethanol.

Chemical and physical properties: Intensely violet in color, although uncertain whether active substance is also colored. Thermostable.

Biological activity: Active against gram-positive bacteria, including micrococci and streptococci, corynebacteria and spore-formers; also active against mycobacteria. Proteins and pus depress activity. Very limited, if any, in vivo activity.

Utilization: None.

Reference: Krassilnikov, N. K., and Koreniako, A. I., Mikrobiologia, 8, 1939, 673; 14, 1945, 80-85; Fainshmidt, O. I., and Koreniako, A. I., Biokhimiya, 9, 1944, 147-153.

\section{Mycomycin}

Produced by: Nocardia acidophilus.

Method of extraction: Ether or amyl acetate.

Chemical and physical properties: Highly unstable unsaturated fatty acid. m.p. $75^{\circ} \mathrm{C}$ (decomposes explosively); $[\alpha]_{\mathrm{D}}^{25}-130^{\circ}$ in ethanol. $\mathrm{C}_{13} \mathrm{H}_{10} \mathrm{O}_{2}$.

Biological activity: Active against bacteria, mycobacteria, and fungi. No activity in vivo.

Toxicity: No reliable data available.

Utilization: None.

Reference: Johnson, E. A., Abstr. papers of the 1949 meeting Soc. Am. Bacteriologists, pp. 68-69; Celmer, W. D., and Solomons, I. A., J. Am. Chem. Soc., 74, 1952, 1870-1871; Jenkins, D. E., Trans. 11th Conf. Chemotherapy of Tuberculosis, Veterans Administration, 1952, 309-310.

\section{Neamine}

Produced by: Streptomyces fradiae.

Synonym: Neomycin A. Neamine is a degradation product of neomycin but might be produced in small quantities by the neomycin-producing organism.

Method of extraction: Acid degradation of neomycin.

Chemical and physical properties: Basic substance; can be separated from neomycin by paper chromatography and countercurrent distribution. Water-soluble; highly diffusible; $[\alpha]_{D}^{25}=+83^{\circ}$ in water. $\mathrm{C}_{6} \mathrm{H}_{12-14} \mathrm{O}_{3} \mathrm{~N}_{2}$. Biological activity: Little activity. Active mainly against gram-positive bacteria.

Toxicity: $\mathrm{LD}_{50}$ (mice), intravenous, $320 \mathrm{mg} / \mathrm{kg}$; subcutaneous, $1250 \mathrm{mg} / \mathrm{kg}$. Utilization: None.

Reference: Peck, R. L., Hoffhine, E. H., Gale, P., and Folkers, K., J. Am. 
Chem. Soc., 71 , 1949, 2590; 75, 1953, 1018; Leach, B. E., and Teeters, C. M., ibid., 7S, 1951, 2794; Leach, B. E., and Teeters, C. M., ibid., $74,1952,3187$.

\section{Neomycin}

Produced by: Streptomyces fradiae and other Streptomyces sp.

Synonyms: Flavomycin. Streptothricin BI is a synonym of neomycin C, and streptothricin BII is a synonym of neomycin B.

Method of extraction: Adsorption on charcoal and elution with acid alcohol. Adsorption on IRC 50 and elution with dilute $\mathrm{HCl}$.

Chemical and physical properties: Basic glycosidic, white, stable substance. Water-soluble, insoluble in organic solvents. No typical absorption spectrum in ultraviolet light. Composed of two isomers, neomycin B and $\mathrm{C}$, which can be separated by chromatography over alumina. Neomycin $\mathrm{B}=[\alpha]_{\mathrm{D}}^{25}+54^{\circ}$, neomycin $\mathrm{C}=[\alpha]_{\mathrm{D}}^{25}+80^{\circ}$. Proximate formula $\mathrm{C}_{29} \mathrm{H}_{58} \mathrm{~N}_{8} \mathrm{O}_{16}$.

Biological activity: Active against gram-positive and gram-negative bacteria, mycobacteria, and actinomycetes. Not active against fungi. Neomycin B is more potent than neomycin C by dilution assay (290 units/mg against 153 units/mg) but does not diffuse in the agar so fast as does neomycin $\mathrm{C}$.

Toxicity: $\mathrm{LD}_{50}$ (mice), subcutaneous, $300-350 \mathrm{mg} / \mathrm{kg}$ (neomycin complex); $220 \mathrm{mg} / \mathrm{kg}$ (neomycin B); $290 \mathrm{mg} / \mathrm{kg}$ (neomycin C).

Utilization: Treatment of bacterial infections of the skin and eyes. Sterilization of the intestine.

Reference: Waksman, S. A., Neomycin, Rutgers University Press, New Brunswick, N. J., 1953; Hamre, D. M., Pansy, F. E., Lapedes, D. N., Perlman, D., Bayan, A. P., and Donovick, R., Antibiotics \& Chemotherapy, 2, 1952, 135-141; Dutcher, J. D., Hosansky, N., Donin, M. N., and Wintersteiner, O., J. Am. Chem. Soc., 73, 1951, 1384.

\section{Neonocardin}

Produced by: Nocardia kuroishi.

Method of extraction: Unknown.

Chemical and physical properties: Found to be different from other known antibiotics by paper chromatography.

Biological activity: Active against Micrococcus pyogenes var. aureus, Escherichia coli, and Bacillus anthracis. Very little activity against Bacillus subtilis and Pseudomonas aeruginosa.

Toxicity: No data.

Utilization: No data.

Reference: Uesaka, I., J. Antibiotics (Japan), C 3, 1950, 27-34; 5, 1952, 75-79; Ueda, S., and Uesaka, I., ibid, 170-171. 


\section{Netropsin}

Produced by: Streptomyces netropsis.

Method of extraction: Broth acidified to $\mathrm{pH} 2.0$ with $\mathrm{H}_{3} \mathrm{PO}_{4}$, filtered on Super-Cel. Filtrate adjusted to $\mathrm{pH} 6.5$ with $\mathrm{NaOH}$ and ammonium oxalate added; precipitate filtered off. Broth then adjusted to $\mathrm{pH} 5.5$ with $\mathrm{HCl}$, and Orange II added. The dye precipitate is filtered off on Super-Cel and washed with distilled water. The air-dry cake is suspended in a mixture of 20 per cent methanol and 80 per cent acetone, and 50 per cent of a methanolic solution of trimethylamine sulfate added. Filtered, washed with acetone-methanol, and extracted with distilled water. The netropsin sulfate solution contains 485 streptomycin units/mg.

Chemical and physical properties: Netropsin sulfate melts at $224-225^{\circ}$ and has a solubility of about $30 \mathrm{mg} / \mathrm{ml}$ in water at $80^{\circ} \mathrm{C}$, but less than 0.5 $\mathrm{mg} / \mathrm{ml}$ at $25^{\circ} \mathrm{C}$. It is quite insoluble in the common organic solvents. The strong basicity of the antibiotic appears to be due to a substituted guanidine group, but this does not account for all of the nitrogen present. Maximum light absorption of sulfate, at pH 5.5, at 236 and $296 \mathrm{~m} \mu ; \mathrm{C}_{32} \mathrm{H}_{48} \mathrm{~N}_{18} \mathrm{O}_{4}$.

Biological activity: Inhibits the growth of various gram-positive and gramnegative organisms, in concentration of $3-40 \mu \mathrm{g} / \mathrm{ml}$. It is effective against clothes moth larvae and the black carpet beetle. Active in experimental mice vaccinal infections. No activity, in mice, against the viruses of feline pneumonitis, influenza, Western equine encephalomyelitis, and poliomyelitis.

Toxicity: $\mathrm{LD}_{50}$ (mice), intravenous, $17 \mathrm{mg} / \mathrm{kg}$; subcutaneous, $70 \mathrm{mg} / \mathrm{kg}$. Utilization: None so far.

Reference: Finlay, A. C., and Sobin, B. A., U. S. Patent 2,586,762, 1952; J. Am. Chem. Soc., 73, 1951, 341; Schabel, F. M., Laster, W. R., Brockman, R. W., and Skipper, H. E., Proc. Soc. Exptl. Biol. Med., 83, 1953, 1-3.

\section{Nigericin}

Produced by: Streptomyces sp.

Method of extraction: Precipitation at $\mathrm{pH} 2$ to 3. Precipitate dissolved in base. Precipitation of salt at $\mathrm{pH}$ 8.5. Can also be extracted from broth with $n$-butanol, butyl acetate, ethyl acetate, or ethyl ether. Can be adsorbed on charcoal and eluted with alcohol.

Chemical and physical properties: Organic acid. Mol. wt. $=715-735$, sodium salt $=$ colorless needles. m.p. $246-254^{\circ} \mathrm{C}$. Soluble in ethyl, methyl, and $n$-butyl alcohols; slightly soluble in water. At $\mathrm{pH} 7.0$, stable 2 hours at $100^{\circ} \mathrm{C} . \mathrm{C}_{39} \mathrm{H}_{69} \mathrm{O}_{11}$. 
Biological activity: Active on gram-positive bacteria $(0.12-0.5 \mathrm{mcg} / \mathrm{ml})$; mycobacteria $(0.5-4.0 \mathrm{mcg} / \mathrm{ml})$, and some fungi, such as $C$. albricans $(2 \mathrm{mcg} / \mathrm{ml}), T$. mentagrophytes $(16 \mathrm{mcg} / \mathrm{ml})$; slightly active against gram-negative bacteria $(64 \mathrm{mcg} / \mathrm{ml})$. Biological activity is inhibited by potassium ions.

Toxicity: $\mathrm{LD}_{60}$ (mice), intraperitoneal, $2.5 \mathrm{mg} / \mathrm{kg}$.

Utilization: None, too toxic.

Reference: Harned, R. L., Hidy, P. H., Corum, C. J., and Jones, K. L., Antibiotics \& Chemotherapy, 1, 1951, 594-596.

\section{Nitrosporin}

Produced by: Streptomyces fasciculus (nitrosporeus).

Synonym: Resembles proactinomycin.

Method of extraction: Adsorption on charcoal and elution with acid acetone. Adsorption on cation exchange resin and elution with dilute acid.

Chemical and physical properties: Basic substance. Maximum light absorption at 250 and $320 \mathrm{~m} \mu$. Soluble in alcohol and ethyl acetate; slightly soluble in ether and ethylene dichloride; insoluble in water but soluble in dilute acids. Stability limited. $\mathrm{C}_{20} \mathrm{H}_{26} \mathrm{~N}_{2} \mathrm{O}_{6}$.

Biological activity: Active mainly against gram-positive bacteria; limited activity against gram-negative bacteria; no activity against mycobacteria and fungi.

Toxicity: $\mathrm{LD}_{50}$ (mice), intravenous, $16 \mathrm{mg} / \mathrm{kg}$.

Utilization: Probably none.

Reference: Umezawa, H., and Takeuchi, T., J. Antibiotics (Japan), 5, 1952, 270-272.

\section{Nocardamin}

Produced by: Nocardia sp. closely related to Nocardia flavescens. Method of extraction: Extraction of the broth with butanol. Butanol concentrated, in vacuo residue extracted with hot water and cooled to yield needle-shaped crystals.

Chemical and physical properties: Reddish substance, soluble in boiling methanol, slightly soluble in hot water. m.p. $183-184^{\circ} \mathrm{C}$. Optically inactive. $\mathrm{C}_{9} \mathrm{H}_{14} \mathrm{~N}_{2} \mathrm{O}_{3}$.

Biological activity: No activity against bacteria and fungi. Active against mycobacteria.

Toxicity: No data.

Utilization: Unknown.

Reference: Stoll, A. von, Brack, A., and Renz, J., Schweiz, Z. Alleem. Pathol. Bakt., 14, 1951, 225-233. 


\section{Nocardianin}

Produced by: Nocardia sp.

Method of extraction: By ether, chromatography on an alumina column, elution with a 50 per cent mixture of chloroform and ethanol. Recrystallization from methanol.

Chemical and physical properties: Red prisms, m.p. $228-235^{\circ} \mathrm{C}$ (decomposition), $[\alpha]_{\mathrm{D}}^{25}-223^{\circ}$. Soluble in chloroform, glacial acetic acid, pyridine; moderately soluble in acetone, methanol; slightly soluble in water and ether; insoluble in petroleum ether, carbon disulfide, carbon tetrachloride. Molecular weight 1386 . No amino acids by acid hydrolysis. Absorption spectrum in methanol: minimum $330 \mathrm{~m} \mu$ and maximum $440 \mathrm{~m} \mu$. Unstable. $\mathrm{C}_{66} \mathrm{H}_{100} \mathrm{O}_{15} \mathrm{~N}_{18}$.

Biological activity: Active against gram-positive bacteria; not active against gram-negative bacteria and mycobacteria.

Toxicity: Unknown.

Utilization: None.

Reference: Bick, I. R., Jann, G. J., and Cram, D. J., Antibiotics \& Chemotherapy, 2, 1952, 255-258.

\section{Nocardin}

Produced by: Nocardia coeliaca.

Method of extraction: Extraction of the mycelium with mixture of ether and 95 per cent alcohol or adsorption from the culture filtrate on charcoal and elution with acid alcohol or a mixture of ether and 95 per cent alcohol.

Chemical and physical properties: Crude nocardin is thermostable and water-soluble.

Biological activity: Active in vivo and in vitro against Mycobacterium tuberculosis. In mice $25 \mathrm{mg}$ of crude nocardin has about the same activity as $1.5 \mathrm{mg}$ of streptomycin. The filtrates of $N$. coeliaca was also active in vitro against Micrococcus pyogenes var. aureus and Bacillus anthracis and to a lesser extent against Bacillus subtilis and Escherichia coli.

Toxicity: $25 \mathrm{mg}$ of crude nocardin much more toxic than $1.5 \mathrm{mg}$ of streptomycin.

Utilization: Probably none.

Reference: Emmart, E. W., Kissling, R. E., and Stark, T. H., J. Bacteriol., 57, 1949, 509-514; Levaditi, C., and Henry, J., Produits Pharm., 4, 1949, 11-14.

\section{Nystatin}

Produced by: Streptomyces noursei.

Synonym: Fungicidin (closely related to antimycoin). 
Method of extraction: Extraction of mycelium with methanol, precipitation by ethyl acetate, re-solution in methanol and precipitation with ether. Chemical and physical properties: Slightly soluble in methanol and ethanol and relatively insoluble in most organic solvents and water. Maximum light absorption at 290,305 , and $316 \mathrm{~m} \mu$. C, 58.1 per cent; $\mathrm{H}, 8.7$ per cent; $\mathrm{N}, 1.8$ per cent.

Biological activity: Not active against bacteria, mycobacteria, and actinomycetes. Active against fungi. Active in vivo in mice against $C$. neoformans, $H$. capsulatum and $C$. immitis.

Toxicity: $\mathrm{LD}_{50}$ (mice), intraperitoneal, $23 \mathrm{mg} / \mathrm{kg}$.

Utilization: Not known.

Reference: Hazen, E. L., and Brown, R., Proc. Soc. Exptl. Biol. Med., 76, 1951, 93-97.

\section{Oxytetracycline}

Produced by: Streptomyces rimosus.

Synonym: Terramycin.

Method of extraction: Adsorption on charcoal at neutrality, elution with acid, eluate adjusted to $\mathrm{pH} 9$ and extracted with butanol.

Chemical and physical properties: Amphoteric substance. Soluble in ethanol, methanol, butanol, and in water mainly at high and low $\mathrm{pH}$. Maximum stability at $\mathrm{pH} 2.5$, half-life of 134 hours at $37^{\circ} \mathrm{C}$. Maximum absorption of ultraviolet light at 265 and $367 \mathrm{~m} \mu$. Bright yellow fluorescence under ultraviolet light. $\mathrm{C}_{22} \mathrm{~N}_{24} \mathrm{~N}_{2} \mathrm{O}_{9}$.

Biological properties: Active against gram-positive, gram-negative, and acid-fast bacteria. Active against rickettsiae. Not active against fungi. Toxicity: $\mathrm{LD}_{50}$ (mice) hydrochloride, intravenous, $178 \mathrm{mg} / \mathrm{kg}$; subcutaneous, $830 \mathrm{mg} / \mathrm{kg}$; oral, $6696 \mathrm{mg} / \mathrm{kg}$.

Utilization: Treatment of numerous bacterial and rickettsial infections. Reference: Ann. N. Y. Acad. Sci., 58, 1950, 223-460; Sobin, B. A., Finlay, A. C., Kane, I. H., U. S. Patent 2,516,080, 1950; Stephens, C. R., and Woodward, R. B., J. Am. Chem. Soc., 74, 1952, 4976-4977.

\section{Phaeofacin}

Produced by: Streptomyces phaeofaciens.

Method of extraction: Extraction of the broth or mycelium with ethyl acetate, concentration in vacuo to a syrup. Syrup dissolved in acetone and cooled with formation of a precipitate.

Chemical and physical properties: Soluble in ethyl acetate, ethanol, acetone, and benzene. Slightly soluble in ether and petroleum ether. Insoluble in water.

Biological activity: Active against Trichophyton, Histoplasma capsulatum, and Cryptococcus neoformans. 
Toxicity: $\mathrm{LD}_{0}$ (mice), intraperitoneal, about $200 \mathrm{mg} / \mathrm{kg}$.

Utilization: None.

Reference: Maeda, K., Okami, Y., Taya, O., and Umezawa, H., J. Antibiotics (Japan) 5, 1952, 465; Japan. J. Med. Sci. Biol., 5, 1952, 327-329.

\section{Phagolessin}

Produced by: Streptomyces sp.

Method of extraction: Precipitation by phosphotungstic acid; extraction with 80 per cent ethanol at $\mathrm{pH} 3.0$ and chromatography over alumina.

Chemical and physical properties: Soluble in acidified water ( $\mathrm{pH} 3.8)$ and methanol; less soluble in ethanol; insoluble in acetone, ether, benzene, chloroform, and petroleum ether. Maximum stability at $\mathrm{pH} 3$, at $100^{\circ} \mathrm{C}$ for 15 minutes. At $\mathrm{pH}$ 9.0, immediate irreversible inactivation occurs. Biological activity: Inhibition of Klebsiella pneumoniae $(3 \mathrm{mcg} / \mathrm{ml})$; other organisms are more resistant, requiring $>12.5 \mathrm{mcg} / \mathrm{ml}$, but some bacteria and mycobacteria are moderately sensitive. Has marked antiphage action.

Toxicity: $\mathrm{LD}_{100}$ (mice), intraperitoneal, $<31 \mathrm{mg} / \mathrm{kg}$.

Utilization: None.

Reference: Asheshov, I. N., Strelitz, F., and Hall, E. A., Antibiotics \& Chemotherapy, 2, 1952, 361-362.

\section{Phalamycin}

Produced by: Streptomyces noursei variant.

Method of extraction: Culture filtrate frozen and thawed. The first $2 / 7$ of the melt contains most of the antibiotic. Extraction from the concentrate at $\mathrm{pH}$ 7.5-8.0 with ethyl acetate which is removed by vacuum distillation. The residue is taken up in methanol, the solution is chilled, and the inactive precipitate formed is discarded. The solution is again concentrated in vacuo, and the residue is picked up in chloroform. The chloroform solution is washed with water and concentrated in vacuo. The residue is washed with ether and extracted with ethyl acetate. Removal of the ethyl acetate leaves phalamycin.

Chemical and physical properties: Yellow powder. Contains nitrogen. Soluble in lower alcohols, esters, chloroform, acetone. Slightly soluble in benzene, ether, and water. Relatively stable from $\mathrm{pH} 2.5$ to 7.0 . Probably neutral. Negative biuret, ninhydrin, Sakaguchi, and Molisch tests. Ultraviolet absorption curve shows a slight inflection between 293 and $316 \mathrm{~m} \mu$.

Biological activity: Active against gram-positive bacteria including mycobacteria and nocardia. Inactive against gram-negative bacteria and fungi. Active in mice against Streptococcus hemolyticus. 
Toxicity: $\mathrm{LD}_{0}$ (mice), subcutaneous, $>115.7 \mathrm{mg} / \mathrm{kg}$; intraperitoneal, $>29$ $\mathrm{mg} / \mathrm{kg}$.

Reference: Brown R., and Hazen, E. L., Antibiotics \& Chemotherapy, 3 , $1953,818-821$.

\section{Picromycin}

Produced by: Streptomyces felleus.

Synonym: Similar to proactinomycin.

Method of extraction: Extraction with ether at weakly alkaline reaction, and further purification by solvent fractionation and chromatography.

Chemical and physical properties: Basic substance having a bitter taste. Unstable. Very soluble in acetone, benzol, chloroform and ethyl acetate. Moderately soluble in ether and cold methanol. Slightly soluble in carbon disulfide, petroleum ether and water. $[\alpha]_{\mathrm{D}}^{24}=+8.2$ (ethanol). No typical ultraviolet absorption spectrum. $\mathrm{C}_{25} \mathrm{H}_{43} \mathrm{NO}_{7}$.

Biological activity: Active against certain gram-positive bacteria. Limited activity against $M$. tuberculosis. Very little activity against $E$. coli and other gram-negative bacteria.

Toxicity: MLD (mice), intravenous, about $150 \mathrm{mg} / \mathrm{kg}$. Utilization: Favorable effect upon bacterial skin infections.

Reference: Brockmann, H., and Henkel, W., Naturwiss., 37, 1950, 138-139;

Brockmann, H., Genth, H., and Stufe, R., Chem. Ber., 85, 1952, 426433; Suhren, O., Med. Klin. (Munich), 46, 1951, 722.

\section{Pleocidin, Pleocidin A}

Produced by: Organism resembling Streptomyces lavendulae.

Synonym: Related to streptothricin.

Method of extraction: Adsorption on Norit A, elution with acid methanol. The filtered broth may also be cleared of $\mathrm{Ca}^{++}$with sodium oxalate and stirred at $\mathrm{pH} 8.0$ with IRC 50 ; the elution is carried out with $\mathrm{HCl}$ and the final neutralization done with IR4B. Further purification by chromatography on Norit A, using methanol as a developer. Pleocidin A can be separated from pleocidin by greater solubility of its helianthate.

Chemical and physical properties: Basic, white hygroscopic powder, maximum stability at $\mathrm{pH} 4$ to 6 .

Biological activity: Active against gram-positive and gram-negative bacteria, mycobacteria, and fungi. The ratio of Escherichia coli to Bacillus subtilis is 64 for pleocidin and 4 for streptothricin. Pleocidin A possesses greater diffusibility than pleocidin, and is less active by dilution assay. Toxicity: $\mathrm{LD}_{50}$ (mice), intraperitoneal, $3 \mathrm{mg} / \mathrm{kg}$. No rabbit's eye toxicity. Utilization: Might find use in topical application. 
Reference: Charney, J., Roberts, W. S., and Fisher, W. P., Antibiotics \& Chemotherapy, 2, 1952, 307-309; Fisher, W. P., and Charney, J., ibid., 311-314.

\section{Proactinomycins A, B, C}

Produced by: Nocardia gardneri.

Method of extraction: Broth $\mathrm{pH}$ adjusted to 10 , extracted with amyl acetate.

Back extracted with acetate buffer at $\mathrm{pH} 4$. $\mathrm{pH}$ adjusted to 10 , extracted with amyl acetate. Back extraction with acid water $(\mathrm{pH} 4$, $\mathrm{HCl})$. Concentration and lyophilization.

Chemical and physical properties: Basic substance. Stable at $\mathrm{pH} 2$ to 8 . Slightly soluble in water at neutrality. Soluble in alcohol, acetone, ether, chloroform, and benzene. This substance can be resolved by countercurrent distribution into three antibiotics:

Proactinomycin A: $\mathrm{C}_{27} \mathrm{H}_{47} \mathrm{NO}_{8}$, m.p. $168-169^{\circ} \mathrm{C}$

Proactinomycin B: $\mathrm{C}_{28} \mathrm{H}_{49} \mathrm{NO}_{8}$, m.p. $83-87^{\circ} \mathrm{C}$

Proactinomycin C: $\mathrm{C}_{24} \mathrm{H}_{41} \mathrm{NO}_{6}$

Biological activity: Active against gram-positive bacteria. Very limited activity against gram-negative bacteria. $\mathrm{A}$ and $\mathrm{C}$ have about the same activity. $\mathrm{B}$ is less active on a weight basis. The three substances have identical qualitative antibacterial spectra. Proactinomycin A offers little protection to mice infected with Streptococcus pmeumoniae.

Toxicity: $\mathrm{LD}_{50}$ (mice), intravenous, $90 \mathrm{mg} / \mathrm{kg}$ (crude proactinomycin); $150 \mathrm{mg} / \mathrm{kg}(\mathrm{A}) ; 120 \mathrm{mg} / \mathrm{kg}$ (B); $80 \mathrm{mg} / \mathrm{kg}$ (C).

Utilization: None.

Reference: Gardner, A. D., and Chain, E., Brit. J. Exptl. Pathol., 23, 1952, 120-127; Marston, R. Q., ibid., 30, 1949, 398-407; Marston, R. Q., and Florey, H. W., ibid., 407-418.

\section{Puromycin}

Produced by: Streptomyces alboniger.

Synonym: Achromycin.

Method of extraction: Not published.

Chemical and physical properties: Nonpigmented basic substance, with dimethylaminopurine, O-methyl-L-tyrosine and 3-amino-D-ribose groups. m.p. $175.5-177^{\circ} \mathrm{C}$. Characteristic infrared absorption. $[\alpha]_{\mathrm{D}}^{25}$ $-11^{\circ}$ in ethanol. Maximum light absorption at $275 \mathrm{~m} \mu$ in $0.1 N \mathrm{NaOH}$. $\mathrm{C}_{22} \mathrm{H}_{29} \mathrm{~N}_{7} \mathrm{O}_{5}$.

Biological activity: Inhibits largely gram-positive bacteria Sarcina lutea, Micrococcus pyogenes var. aureus, Bacillus subtilis, Klebsiella pneumoniae $(2-8 \mathrm{mcg} / \mathrm{ml})$; limited activity against Escherichia coli, Pro- 
teus vulgaris $(200-400 \mathrm{mcg} / \mathrm{ml})$, and Mycobacterium $607(40 \mathrm{mcg} / \mathrm{ml})$.

Active in mice against Trypanosoma equiperdum.

Toxicity: $\mathrm{LD}_{50}$ (mice), intravenous, $350 \mathrm{mg} / \mathrm{kg}$; intraperitoneal, 525 $\mathrm{mg} / \mathrm{kg}$; oral, $675 \mathrm{mg} / \mathrm{kg}$.

Utilization: Might be used on an antiprotozoal agent.

Reference: Porter, J. N., Hewitt, R. I., Hesseltine, C. W., Krupka, G., Lowery, J. A., Wallace, W. S., Bohonos, N., and Williams, J. H., Antibiotics \& Chemotherapy, 2, 1952, 409-410; Waller, C. W., Fryth, P. W., Hutchings, B. L., and Williams, J. H., Am. Chem. Soc. 75, 1953, 2025; Baker, B. R., and Schaub, R. E., ibid., 3864.

\section{Resistomycin}

Produced by: Streptomyces resistomycificus.

Method of extraction: Mycelium extracted with petroleum ether, then with ether, the latter giving an active yellow extract. Chromatographic separation yields dioxane-soluble, saffron-yellow, needle-shaped crystals.

Chemical and physical properties: Soluble in water with difficulty. Soluble in ether, ethanol, and other organic solvents. Weak acid. Resistant to action of alkali, acid, and heat $\left(250^{\circ} \mathrm{C}\right)$. It contains no $\mathrm{N}$ or S. It decomposes, without melting, at $315^{\circ} \mathrm{C} . \mathrm{C}_{23} \mathrm{H}_{18} \mathrm{O}_{6}$.

Biological activity: Active against Micrococcus pyogenes var.aureus and Bacillus subtilis in dilution of 1:20,000,000, and against Myocobacterium tuberculosis in dilution of $1: 500,000$ to $1: 1,000,000$.

Toxicity: No information.

Utilization: No information.

Reference: Brockmann, H., and Schmidt-Kastner, G., Naturwiss., 38, 1951, 479-480.

\section{Rhodocidin}

Produced by: Streptomyces phoenix (no description available).

Method of extraction: Addition of 0.03 per cent $\mathrm{H}_{2} \mathrm{O}_{2}$ to broth. Filtration of the mycelium. Addition of 15 per cent $\mathrm{NaCl}$, extraction with ethyl acetate. Concentration, addition of petroleum ether, extraction with water. Addition of 2 per cent $\mathrm{NaHSO}_{3}$, washing with ethyl acetate. After addition of 2 per cent acetone, extraction with ethyl acetate. Addition of petroleum ether, extraction with water, concentration, lyophilization.

Chemical and physical properties: Red powder, showing a broad peak of absorption at $500-530 \mathrm{~m} \mu$. Soluble in water and in many organic solvents. Inactivated rapidly by dilute acids and alkalies. Inactivated 
by the mycelium of $S$. phoenix in absence of excess oxygen. Organic solvent solutions and dry powders are stable indefinitely at $55^{\circ} \mathrm{C}$.

Biological activity: Active against mycobacteria, gram-positive, and gramnegative bacteria. It is active in vivo in mice against Streptococcus pyogenes. It is much more active by the intraperitoneal route than by the intravenous or intramuscular routes.

Toxicity: $\mathrm{LD}_{50}$ (mice), intraperitoneal, $2.1 \mathrm{mg} / \mathrm{kg}$; intravenous, $2 \mathrm{mg} / \mathrm{kg}$; intramuscular, $3 \mathrm{mg} / \mathrm{kg}$.

Utilization: Probably none.

Reference: Charney, J., Machlowitz, R. A., Roberts, W. S., and Fisher, W. P., Antibiotics \& Chemotherapy, 3, 1953, 788-792.

\section{Rhodomycetin}

Produced by: Red mutant of Streptomyces griseus.

Method of extraction: Precipitation with acid and extraction of precipitate with methanol; also extraction of broth with $n$-butanol.

Chemical and physical properties: Red in acid solutions and blue at alkaline reactions. Unstable under alkaline conditions. Can stand 10 minutes at $70^{\circ} \mathrm{C}$ at $\mathrm{pH}$ 7.0. Insoluble in water; soluble in ethylene glycol monomethyl ether, ether, acetone, acetic acid, and various alcohols. Maximum light absorption at 235, 540, and $580 \mathrm{~m} \mu$.

Biological activity: Active against gram-positive bacteria, moderately active against mycobacteria, almost no activity against gram-negative bacteria. No activity in mice.

Toxicity: No clear data available.

Reference: Shockman, G., and Waksman, S. A., Antibiotics \& Chemotherapy, 1, 1951, 68-75.

\section{Rhodomycin}

Produced by: Streptomyces sp.

Method of extraction: From aqueous solutions of both broth and mycelium at $\mathrm{pH} 8.6$ with butanol, chloroform, or ether.

Chemical and physical properties: Amphoteric dark red pigment acting as a $\mathrm{pH}$ indicator. As the chloride or phosphate, it is water-soluble. As a picrate, it is water-insoluble. Soluble in organic solvents. Maximum light absorption in benzene at 575, 542, 533, and $504 \mathrm{~m} \mu$. Unstable at acid and alkaline $\mathrm{pH} . \mathrm{C}_{22} \mathrm{H}_{29} \mathrm{NO}_{7}$. Through mild acid hydrolysis, rhodomycin can be split into a basic, nonpigmented, water-soluble substance and a water-insoluble pigment. The pigment is an oxynaphthoquinone $\left(\mathrm{C}_{15} \mathrm{H}_{14} \mathrm{O}_{6}\right)$.

Biological activity: Rhodomycin stops growth of Micrococcus pyogenes var. 
aureus at dilution of $1 / 50$ million; the pigmented component, at dilution of $1 / 300,000$.

Toxicity: No data.

Utilization: None.

Reference: Brockmann, H., and Bauer, K., Naturwiss., 2, 1950, 492-493;

Brockmann, H., and Borchers, I., Chem. Ber., 86, 1953, 261-269.

\section{Rimocidin}

Produced by: Streptomyces rimosus.

Method of extraction: Extraction of the mycelium with $n$-butanol.

Chemical and physical properties: Amphoteric substance. Analysis: C,

57.6 per cent; $H, 7.8$ per cent; $N, 1.8$ per cent; $S, 2.0$ per cent. $[\alpha]_{\mathrm{D}}^{25}=$ $+75.2^{\circ}$; m.p. $151^{\circ} \mathrm{C}$ (decomposition). Maximum ultraviolet absorption at $279,291,304$, and $318 \mathrm{~m} \mu$.

Biological activity: Active in concentration of $3 \mathrm{mcg} / \mathrm{ml}$ against Candida albicans, Histoplasma capsulatum, and many other pathogenic fungi.

C. neoformans is more resistant.

Toxicity: $\mathrm{LD}_{50}$ (mice), intravenous, $30 \mathrm{mg} / \mathrm{kg}$.

Utilization: Not known yet, if any.

Reference: Davisson, J. W., Tanner, F. W. Jr., Finlay, A. C., and Solomons,

I. A., Antibiotics \& Chemotherapy, 1, 1951, 289-290.

\section{Roseomycin}

Produced by: Streptomyces roseochromogenus.

Method of extraction: Same as streptomycin and streptothricin.

Chemical and physical properties: Basic substance similar to streptothricin and streptomycin.

Biological activity: Active against gram-positive bacteria, gram-negative bacteria, and mycobacteria. Very little activity against clostridia.

Active against Vibrio comma. Active in vivo against Eberthella typhosa. Toxicity: $\mathrm{LD}_{0}$ (mice), intravenous and intramuscular, about $1000 \mathrm{mg} / \mathrm{kg}$. Utilization: Not known yet.

Reference: Ishida, N., J. Antibiotics (Japan), 3, 1950, 839-853; Nagao, I., ibid., C $3,1950,20-33$; 4, 1951, 24-28.

\section{Rotaventin}

Produced by: Streptomyces reticuli.

Method of extraction: Extraction of mycelium with methanol. Concentration in vacuo. Precipitation at $\mathrm{pH}$ 2.0. Precipitate washed with ether. Residue dissolved in methanol and precipitated with water. The final purification is accomplished by countercurrent distribution. 
Chemical and physical properties: Rotaventin forms colorless platelets, changing to yellow at about $130^{\circ} \mathrm{C}$; m.p. $170-175^{\circ} \mathrm{C}$. Soluble in methanol, butanol, amyl alcohol, and acetone. Insoluble in ether, petroleum ether, chloroform, benzene, ethyl acetate, amyl acetate, trichloroethylene, 5 per cent sodium bicarbonate, water at $\mathrm{pH} 2.0$. Solution of rotaventin in methanol or ethanol cannot be precipitated by phosphotungstic acid and picric acid. Rotaventin changes to yellow in air gradually, to red when treated with concentrated $\mathrm{H}_{2} \mathrm{SO}_{4}$. $\mathrm{FeCl}_{2}$ reaction, negative.

Biological activity: Active in vitro against Saccharomyces sake, Aspergillus niger, and Penicillium glaucum in concentration of $6 \mathrm{mcg} / \mathrm{ml}$. Little or no activity against Candida albicans, Mucor, Rhizopus, and Trichopyton. No activity against bacteria.

Toxicity: $\mathrm{LD}_{0}$ (mice), intraperitoneal, about $150 \mathrm{mg} / \mathrm{kg}$; subcutaneous, about $770 \mathrm{mg} / \mathrm{kg}$.

Utilization: Not known.

Reference: Hosoya, S., Komatsu, N., and Soeda, M., J. Antibiotics( Japan), $5,1952,451-453$.

\section{Sarcidin}

Produced by: Streptomyces achromogenes.

Method of extraction: Adsorption on charcoal at $\mathrm{pH}$ 7.0. Elution with acid alcohol, concentration in vacuo, crystallization at 0 to $5^{\circ} \mathrm{C}$, recrystallization from 80 per cent aqueous ethanol.

Chemical and physical properties: m.p. $274-275^{\circ} \mathrm{C}$ with decomposition. C 41.9 per cent, H 5.0 per cent, N 21.8 per cent. Halogen and sulfur might also be present. Maximum stability at $\mathrm{pH}$ 2.0.

Biological activity: Active against Sarcina lutea in concentration of 1-1.5 $\mathrm{mcg} / \mathrm{ml}$. No activity against a great number of bacteria and fungi tested.

Toxicity: $\mathrm{LD}_{0}$ (mice), intraperitoneal, $>660 \mathrm{mg} / \mathrm{kg}$.

Utilization: No data.

Reference: Takeuchi, T., Nitta, K., and Umezawa, H., J. Antibiotics (Japan), A 6, 1953, 31-32.

\section{Sarkomycin}

Produced by: Streptomyces sp. closely related to S. erythrochromogenes.

Method of extraction: Extraction of broth at $\mathrm{pH} 2.0$ with butanol, back extraction in water at $\mathrm{pH}$ 7.0. Adsorption on charcoal at $\mathrm{pH} 2.0$, elution with 80 per cent ethanol at $\mathrm{pH} 7.0$.

Chemical and physical properties: Crude powder soluble in water and 
methanol and slightly soluble in ethanol, butanol and acetone. Biuret, ninhydrin, Molisch and Benedict's tests negative.

Biological activity: Slight activity against, Micrococcus pyogenes var. aureus, no activity against $B$. subtilis, $E$. coli and $C$. albicans. In vivo action against Yoshida sarcoma in rats and Ehrlich carcinoma in mice.

Toxicity: $\mathrm{LD}_{50}$ (mice), intravenous, more than $2,400 \mathrm{mg} / \mathrm{kg}$.

Utilization: Not known.

Reference: Umezawa, H., Takeuchi, T., and Nitta, K., J. Antibiotics (Japan), A 6, 1953, 101.

\section{Streptin}

Produced by: Streptomyces sp. resembling S. lavendulae and S. reticulus-ruber. Method of extraction: Same as streptothricin.

Chemical and physical properties: Basic, water-soluble substance.

Biological activity: Active against gram-positive bacteria, gram-negative

bacteria, and mycobacteria. More active against staphylococci and micrococci than are streptomycin and streptothricin.

Toxicity: No data.

Utilization: None.

Reference: Woodruff, H. B., and Foster, J. W., J. Bacteriol., 52, 1946, 502.

\section{Streptocin}

Produced by: Streptomyces griseus.

Method of extraction: Extraction of the mycelium with diethyl ether. Concentration of ether in vacuo, extraction of residue with ethanol. Concentration of ethanol, extraction with petroleum ether. Chromatography on alumina and elution with 1 per cent acetic acid in methanol yield needle-like crystals.

Chemical and physical properties: Soluble in water, methanol, and ethanol but not in chloroform. Stable. Sublimable.

Biological activity: Slightly active against gram-positjve bacteria and mycobacteria. Active against trichomonads.

Toxicity: $\mathrm{LD}_{0}$ (mice), intravenous, $>800 \mathrm{mg} / \mathrm{kg}$.

Reference: Waksman, S. A., Harris, D. A., Kupferberg, A. B., Singher, H. O., and Styles, H., Proc. Soc. Exptl. Biol. Med., 70, 1949, 308-312; Kupferberg, A. B., Styles, H., Singher, H. O., and Waksman, S. A., J. Bacteriol., 59, 1950, 523-526.

\section{Streptolin}

Produced by: Streptomyces sp.

Method of extraction: Adsorption on and elution from Darco G60. 
Chemical and physical properties: Basic, water-soluble substance. Most stable at $\mathrm{pH} 3.0-3.5 ;[\alpha]_{\mathrm{D}}^{25}$ (hydrochloride) $-22^{\circ}$. Similar to streptothricin. Can be separated by paper chromatography into two active components, A and B. Streptolin A sulfate has the empirical formula $\mathrm{C}_{17} \mathrm{H}_{31-33} \mathrm{~N}_{5} \mathrm{O}_{8}$ or $\mathrm{C}_{24} \mathrm{H}_{45} \mathrm{~N}_{7} \mathrm{O}_{11}$ for the free base. Acid hydrolysis of streptolin indicates that it contains an amino sugar, a basic substance, and an isomer of lysine (isolysine).

Biological activity: Active against gram-positive and gram-negative bacteria. Toxicity: $\mathrm{LD}_{0}$ (mice), intravenous, about $4.5 \mathrm{mg} / \mathrm{kg} ; \mathrm{LD}_{100}$, about 13.5 $\mathrm{mg} / \mathrm{kg}$.

Utilization: None.

Reference: Rivett, R. W., and Peterson, W. H., J. Am. Chem. Soc., 69, 1947, 3006-3009; Larson, L. M., Sternberg, H. E., Peterson, W. H., Smissman, E. E., Sharpe, R. W., and Tamelen, E. E. van., Abstr. of papers 121 Meeting, Am. Chem. Soc., 1952, p. 8C; J. Am. Chem. Soc., 75, 1953, 2029-2031; ibid., 2036-2039.

\section{Streptomycin}

Produced by: Streptomyces griseus and S. bikiniensis.

Synonym: Streptomycin II.

Method of extraction: Adsorption on activated charcoal and elution with acid alcohol. Adsorption on IRC 50 and elution with dilute $\mathrm{HCl}$.

Chemical and physical properties: Basic, white, stable substance. Watersoluble, insoluble in organic solvents. Inactivated by cysteine and hydroxylamine. Heteroglucoside, composed of an organic base, streptidine, and of two sugar moieties forming streptobiosamine. $\mathrm{C}_{21} \mathrm{H}_{39} \mathrm{~N}_{7} \mathrm{O}_{12}$.

Biological activity: Active against gram-positive, gram-negative, and acidfast bacteria. Not active against fungi, rickettsiae, and viruses.

Toxicity: $\mathrm{LD}_{50}$ (mice), subcutaneous, $900 \mathrm{mg} / \mathrm{kg}$; intravenous, $200 \mathrm{mg} / \mathrm{kg}$; oral, $9000 \mathrm{mg} / \mathrm{kg}$. Vestibular disturbances are more common with streptomycin than with dihydrostreptomycin.

Utilization: Treatment of tuberculosis mainly in combination with paraaminosalicylic acid and isoniazid. Treatment of various infections caused by gram-negative and gram-positive bacteria.

References: Waksman, S. A., Streptomycin, Williams and Wilkins, 1949; Waksman, S. A., Literature on Streptomycin, Rutgers University Press, 1952.

\section{Streptomycin-Isoniazid Complex}

Produced by: Chemical derivative of streptomycin and isoniazid. Synonym: Streptohydrazid. 
Production: Streptomycin-isoniazid complex, or streptohydrazid in the form of the hydrochloride is prepared by boiling under reflux for 15 minutes in absolute methanol a mixture of 5 parts of streptomycin hydrochloride and 1 part of isoniazid. Upon standing in the refrigerator large crystals are slowly formed. In order to prepare the sulfate, a mixture of streptomycin trihydrochloride calcium chloride double salt, isoniazid and triethylamine sulfate is stirred in aqueous methanolic solution for 30 minutes. The precipitated $\mathrm{CaSO}_{4}$ is removed. Methanol is added slowly as the white crystalline product is formed.

Chemical and physical properties: The hydrochloride and sulfate of the streptomycin-isoniazid complex are extremely water soluble and insoluble in most organic solvents. Chemically they are streptomycylideneisonicotinylhydrazine trihydrochloride $\left(\mathrm{C}_{27} \mathrm{H}_{47} \mathrm{~N}_{10} \mathrm{O}_{12} \mathrm{Cl}_{3}\right)$ and streptomycylideneisonicotinylhydrazine sesquisulfate $\left(\mathrm{C}_{23} \mathrm{H}_{44} \mathrm{~N}_{10} \mathrm{O}_{12} \cdot 3 / 2 \mathrm{H}_{2} \mathrm{SO}_{4}\right)$.

Biological activity: Streptohydrazid therapy has been found to be as effective in tuberculosis protection studies in animals as combined streptomycinisoniazid therapy.

Utilization: Recommended in the treatment of various forms of tuberculosis.

Reference: Pennington, F. C., Guercio, P. A., and Solomons, I. A., J. Am. Chem. Soc., 75, 1953, 2261.

\section{Streptothricin}

Produced by: Streptomyces lavendulae.

Synonyms: The following antibiotics are closely related to streptothricin: streptin, lavendulin, streptolin, streptothricin VI, aerothricin and pleocidin.

Method of extraction: Adsorption on IRC 50 and elution with acid alcohol. Adsorption on Decalso and elution with $\mathrm{NH}_{4} \mathrm{Cl}$.

Chemical and physical properties: Basic substance. Not inactivated by cysteine. Contains two amino acids including isolysine and an amino sugar. Soluble in water, insoluble in organic solvents except methanol. Stable. $\mathrm{C}_{20} \mathrm{H}_{36} \mathrm{~N}_{8} \mathrm{O}_{9} \cdot 3 \mathrm{HCl}$.

Biological activity: Active against gram-positive and gram-negative bacteria, mycobacteria, and certain fungi. Not active against rickettsiae and viruses. Little activity against Bacillus cereus-mycoides group. Toxicity: $\mathrm{LD}_{50}$ (mice), intravenous, $250 \mathrm{mg} / \mathrm{kg}$. High delayed toxicity. Utilization: Possible use in topical application.

Reference: Waksman, S. A., and Woodruff, H. B., Proc. Soc. Exptl. Biol. Med., 49, 1942, 207-210; Hutchison, D., Swart, E. A., and Waksman, S. A., Arch. Biochem., 22, 1949, 16-30; Molitor, H., Ann. N. Y. Acad. Sci., 48 (art. 2), 1946, 101-118. 


\section{Sulfactin}

Produced by: Streptomyces roseus.

Method of extraction: Acidification of culture filtrate to $\mathrm{pH} \mathrm{3}$, extraction with butanol, concentration in vacuo, residue washed with boiling ether then extraction with chloroform. The chloroform extract could be further purified by chromatographing on florisil. Crystallization from boiling alcohol.

Chemical and physical properties: White crystals, m.p. $245-275^{\circ} \mathrm{C}$, with decomposition. Soluble in chloroform, ethanol, ethyl acetate, dioxane, and butanol. Almost insoluble in water, ether, petroleum ether, and benzene. $\mathrm{C}_{38} \mathrm{H}_{55} \mathrm{~N}_{11} \mathrm{O}_{7} \mathrm{~S}_{4}$.

Biological activity: Active mainly against certain gram-positive bacteria. Not active against gram-negative bacteria and mycobacteria. Active in mice against Diplococcus pneumoniae.

Toxicity: $\mathrm{LD}_{50}$ (mice), intraperitoneal, $135 \mathrm{mg} / \mathrm{kg}$.

Utilization: None.

Reference: Junovicz-Kocholaty, R., Kocholaty, W., and Kelner, A., J.

Biol. Chem., 168, 1947, 765-769; Morton, H. E., Proc. Soc. Exptl. Biol. Med., 66, 1947, 345-348.

\section{Thermomycin}

Produced by: Streptomyces thermophilus (S form).

Method of extraction: Salting out with ammonium sulfate or extraction with ether.

Chemical and physical properties: Heating 15 minutes at $100^{\circ} \mathrm{C}$ destroys thermomycin; 15 minutes at $75^{\circ}$ destroys 25 per cent of the activity.

Biological activity: Active mainly against Corynebacterium diphtheriae.

Toxicity: No data.

Utilization: No data.

Reference: Schone, R., Antibiotics \& Chemotherapy, 1, 1951, 176-180.

\section{Thioaurin}

Produced by: Streptomyces sp.

Synonym: Similar to antibiotic HA-9.

Method of extraction: Extraction of filtered broth with ethyl acetate. Concentration in vacuo. Residue dissolved in carbon tetrachloride. Carbon tetrachloride solution extracted with water, which is back extracted with ethyl acetate. Upon concentration in vacuo and cooling, crystallization occurs. Recrystallization from acetone.

Chemical and physical properties: Golden yellow, needle-shaped crystals. Relatively insoluble in water and organic solvents. m.p. $179-181^{\circ} \mathrm{C}$, 
with decomposition. Maximum ultraviolet absorption at 232 and 370 $\mathrm{m} \mu . \mathrm{C}_{14} \mathrm{H}_{12} \mathrm{O}_{4} \mathrm{~N}_{4} \mathrm{~S}_{4}$.

Biological activity: Active against gram-positive and gram-negative bacteria. Relatively inactive against fungi.

Toxicity: $\mathrm{LD}_{50}$ (mice), intravenous, $16 \mathrm{mg} / \mathrm{kg}$.

Utilization: Little promise, because of toxic nature.

Reference: Bolhofer, W. A., Machlowitz, and Charney, J., Abstr. of paper

122 Meeting Am. Chem. Soc., 1952, p. 12A; Antibiotics \& Chemotherapy, 3 , 1953, 383-384, 385-392.

\section{Thiolutin}

Produced by: Streptomyces albus, S. celluloflavus.

Synonyms: Aureothricin, farcinicin.

Method of extraction: The broth is adjusted to $\mathrm{pH} 2.0$ with $\mathrm{HCl}$ and filtered. The clear filtrate is adjusted to $\mathrm{pH} 7.0$ with $\mathrm{NaOH}$ and extracted by stirring with $1 / 2$ or $1 / 4$ volume of ethyl acetate. After removal of the ethyl acetate layer, the extraction is repeated once. The combined ethyl acetate extract is dehydrated with anhydrous sodium sulfate. After sodium sulfate is removed by filtration, ethyl acetate is concentrated in vacuo at $30-40^{\circ} \mathrm{C}$. In the course of concentration there appears a yellow crystalline substance. After recrystallization from ethyl acetate, yellow crystals decomposing at $256-257^{\circ} \mathrm{C}$ are obtained.

Chemical and physical properties: Neutral, optically inactive, yellow-orange crystals of a high sulfur content. Slightly soluble in water, ether, benzene; soluble in ethanol, methanol, chloroform, acetone, glacial acetic acid, methyl isobutyl ketone. Maximum light absorption at 365,315 , and $245 \mathrm{~m} \mu$; minimum at 235,285 , and $330 \mathrm{~m} \mu$; decomposes at $270^{\circ} \mathrm{C}$. Stable in acid and neutral solution; decomposes in alkaline solutions. $\mathrm{C}_{8} \mathrm{H}_{8} \mathrm{~N}_{2} \mathrm{~S}_{2} \mathrm{O}$ (aureothricin- $\mathrm{C}_{9} \mathrm{H}_{10} \mathrm{~N}_{2} \mathrm{~S}_{2} \mathrm{O}_{2}$ ).

Biological activity: Active against gram-positive and gram-negative bacteria in concentration of 1 to $15 \mathrm{mcg} / \mathrm{ml}$, Pseudomonas and Brucella being resistant. Pathogenic fungi inhibited in concentration of $1-5 \mathrm{mcg} / \mathrm{ml}$. Candida albicans resistant. Mild but definite microbicidal action against bacteria, fungi, and protozoa.

Toxicity: $\mathrm{LD}_{50}$ (mice), subcutaneous, $25 \mathrm{mg} / \mathrm{kg}$; oral, $25 \mathrm{mg} / \mathrm{kg}$.

Utilization: Recommended for topical use against fungus infections.

Reference: Seneca, H., Kane, J. H., and Rockenbach, J., Antibiotics \& Chemotherapy, 2, 1952, 357-360; Celmer, W. D., Tanner, F. W., Jr., Harfenist, M., Lees, T. M., and Solomons, I. A., J. Am. Chem. Soc., 74, 1952, 6304-6305; Umezawa, H., Maeda, K., and Kosaka, H., Japan Med. J., 1, 1948, 512-517. 


\section{Trichomycin}

Produced by: Streptomyces hachijoensis.

Method of extraction: Extraction of mycelial mat with acetone or methanol, extract concentrated in vacuo, $\mathrm{pH}$ adjusted to 5.4, giving a yellow precipitate.

Chemical and physical properties: Unstable at acid reaction, stable in weak alkali; soluble in butanol, ethanol, acetone; very soluble in water at alkaline $\mathrm{pH}$; insoluble in ether, petroleum ether, and ethyl acetate. Gives blue color when treated with conc. mineral acids. Maximum absorption of ultraviolet light at 235 and $335 \mathrm{~m} \mu$.

Biological activity: No activity against bacteria; weak activity against filamentous fungi. Active against yeasts, including Candida albicans, and two species of Trichomonas.

Toxicity: $\mathrm{LD}_{50}$ (mice), subcutaneous, $>125 \mathrm{mg} / \mathrm{kg}$; intraperitoneal, $5 \mathrm{mg} / \mathrm{kg}$. Utilization: Most likely none.

Reference: Hosoya, S., Komatsu, N., Soeda, M., Yuwaguchi, T., and Sonoda, Y., J. Antibiotics (Japan), 5, 1952, 564-566.

\section{Vinacetin}

Produced by: Streptomyces sp. Closely related to $S$. albosporeus.

Method of extraction: Broth adjusted to $\mathrm{pH} \mathrm{5.0,} \mathrm{filtered} \mathrm{on} \mathrm{paper} \mathrm{to} \mathrm{remove}$ inert precipitate. Adsorption on cation exchange resin, elution with acetone. Evaporation of acetone, extraction of residual aqueous solution with ethyl acetate. Evaporation in vacuo of the ethyl acetate leaves a brown powder. The pure antibiotic can be crystallized from a chloroform-ether mixture.

Chemical and physical properties: Yellow platelet shaped crystals. m.p. $157-158^{\circ} \mathrm{C}$. Soluble in ethyl acetate, butyl acetate, methanol and acetone. Slightly soluble in water, insoluble in ether and petroleum ether. Soluble in alkaline water with development of a violet color. More active and more stable at $\mathrm{pH} 5.0$ than at $\mathrm{pH} 7.0$ and 9.0. Negative ninhydrin, Sakaguchi's and Millon's reactions. Positive Molisch, $\mathrm{FeCl}_{3}$ and Fehling's reactions.

Biological activity: Active against certain gram-positive bacteria (Staph. citreus, Corynebacterium diphtheriae) and mycobacteria. Inactive against gram-negative bacteria and fungi.

Toxicity: $5 \mathrm{mg}$ per $15 \mathrm{gm}$ mouse injected intravenously and intraperitoneally had no toxic effect. $10 \mathrm{mg}$ killed the mice.

Utilization: Unknown.

Reference: Omachi, K., J. Antibiotics (Japan), A 6, 1953, 73-79. 


\section{Vinactin A, B, C}

Produced by: Streptomyces vinaceus.

Synonym: Probably viomycin.

Method of extraction: Adsorption on Norit and elution with acid methanol.

Precipitation with petroleum ether.

Chemical and physical properties: Vinactin can be separated into three fractions, A, B, and C, which are peptides. Vinactin A and B are composed of lysine, serine, alanine, glycine, glutamic acid, and aspartic acid. Vinactin $\mathrm{C}$ is composed of serine, sarcosine, glycine, and an unidentified amino acid.

Biological activity: Very active against mycobacteria. Active against various gram-positive and gram-negative bacteria and rickettsiae.

Toxicity: No data.

Utilization: None to date.

Reference: Mayer, R. L., Crane, C., DeBoer, C. J., Konopka, E. A., Marsh, J. S., Eisman, P. C., Townley, R. W., Mull, R. P., and Scholz, C. R., Abstr. papers 12th Intern. Congr. Pure Appl. Chem., New York, 1951, pp. 283-284.

\section{Viomycin}

Produced by: Streptomyces floridae, S. juniceus, and S. vinaceus.

Synonyms: Viocin, probably vinactin.

Method of extraction: Adsorption on and elution from active carbon followed by repeated precipitation from aqueous methanol.

Chemical and physical properties: Basic substance; resembles a polypeptide; contains isolysine. Stable mainly at acid $\mathrm{pH}$. Maximum ultraviolet light absorption at $268.5 \mathrm{~m} \mu$ at $\mathrm{pH}$ 7.0. Very soluble in water, insoluble in organic solvents. $\mathrm{C}_{18} \mathrm{H}_{31-33} \mathrm{~N}_{9} \mathrm{O}_{8}$.

Biological activity: Active against mycobacteria $(0.78-12.5 \mathrm{mcg} / \mathrm{ml})$, little activity against most bacteria $(10->100 \mathrm{mcg} / \mathrm{ml})$. More active at alkaline than at acid $\mathrm{pH}$.

Toxicity: In mice, single doses of the sulfate $250 \mathrm{mg} / \mathrm{kg}$ intravenously and $100 \mathrm{mg} / \mathrm{kg}$ subcutaneously cause no fatality.

Utilization: Treatment of tuberculosis in desperate cases.

Reference: Bartz, Q. R., Ehrlich, J., Mold, J. D., Penner, M. A., and Smith, R. M., Am. Rev. Tuberc., 63, 1951, 4; Haskell, T. H., Fusari, S. A., Frohardt, R. P., and Bartz, Q. R., J. Am. Chem. Soc., 74, 1952, 599.

\section{Xanthomycins}

Produced by: Streptomyces sp.

Method of extraction: Adsorption from broth at $\mathrm{pH} 7.5$ to 8.0 with Norit. 
Elution with $0.1 \mathrm{~N} \mathrm{HCl}$ saturated with butanol. Eluate neutralized and extracted with chloroform. Solvent back extracted with acid water ( $\mathrm{pH}$ 2.0). Antibiotic precipitated from the water solution with picric acid or ammonium reineckate. Twenty-four transfer countercurrent distribution between chloroform and phosphate buffer, at $\mathrm{pH}$ 4.4, separates xanthomycin B (tubes 1-4) and xanthomycin A (tubes $12-20$ ). A third component, xanthomycin $\mathrm{C}$, is relatively inactive and is present in tubes 23-24. Xanthomycin A makes up 65 to 90 per cent of the total antibiotic.

Chemical and physical properties: Basic compounds, solvent-soluble, yellow at acid $\mathrm{pH}$, pink at alkaline reaction. Xanthomycin $\mathrm{A}$ hydrochloride is a yellow amorphous material very soluble in water and methanol. Maximum ultraviolet light absorption at 265, $326 \mathrm{~m} \mu$ in water. Maximum stability at acid reaction.

Biological activity: Active against gram-positive and gram-negative bacteria. Very limited activity against mycobacteria.

Toxicity: $\mathrm{LD}_{50}$ (mice), intravenous, about $0.16 \mathrm{mg} / \mathrm{kg}$.

Utilization: None.

References: Thorne, C. B., and Peterson, W. H., J. Biol. Chem., 176, 1948, 413-428; Rao, K. V., and Peterson, W. H., Abstr. papers 12th Intern. Congr. Pure Appl. Chem., New York, 1951, p. 282. 


\section{In Retrospect}

Since the discovery of the great potentialities of antibiotics as chemotherapeutic agents, interest in the actinomycetes has greatly increased. Following the rediscovery of penicillin, the most important antibiotics discovered during the past 10 years and utilized in the treatment of human, animal and even certain plant diseases, as well as in the nutrition of nonherbivorous animals, are produced by actinomycetes. At least ten such compounds are now being used clinically on a large scale. Several others are on their way.

Most of the antibiotics so far isolated are active largely upon bacteria and actinomycetes; some are active upon fungi, a few upon protozoa or viruses. Our knowledge of anti-tumor properties of antibiotics of actinomycetes is still very limited; there is no doubt, however, that such substances exist, as in the case of the cytostatic action of some of the preparations; how soon they may become effective as chemotherapeutic agents still remains to be determined. We know already that just as the antibacterial and antifungal substances are characterized primarily by their specific spectra or range of activity upon different organisms, so are the true antiviral and anti-tumor agents.

An attempt has been made in the guide to bring together the descriptions of the various well-authenticated cultures of actinomycetes, so as to aid in their identification, as well as to summarize the information of the antibiotics produced by them, without any exhaustive treatment of either of these two phases of our knowledge of actinomycetes and their biochemical potentialities.

Many species of actinomycetes listed in the literature have so far been only incompletely described. A line had to be drawn, however, between a complete or exhaustive description of a species and an extremely poor or insufficient description. In selecting the cultures for inclusion in this guide, consideration has been given to the significance of the substrate from which they were isolated, their particular morphology, their important physiological properties or their ability to produce specific antibiotics.

Although an attempt has been made to include all the antibiotics produced by actinomycetes, certain limitations have been encountered here as well. Many of the preparations are only incompletely described. Some are no doubt synonyms, but they cannot be recognized at this time. Many preparations have been referred to in the scientific literature or in the press, but their descriptions have not become available to the authors at the time of writing. It is known, for example, that several antibiotics have been 
announced in the Russian literature, such as albomycin; other preparations have been announced in Japan, such as cremomycin, fuscamycin, orientomycin, podomycin, flavacid.

It is the hope of the authors that in spite of the above limitations, this guide will thus make available the rapidly accumulating information on the classification and characterization of the actinomycetes, a group of organisms that have now become famous because of their ability to produce a most interesting and valuable group of metabolic products, namely the antibiotics. 


\section{Selected}

\section{General References}

1. Alexopotlos, C. J., And Herrick, J. A. 1942 Studies in antibiosis between bacteria and fungi. III. Inhibitory action of some actinomycetes on various species of fungi in culture. Bull. Torrey Botan. Club, 69: 257-261.

2. Batdacci, E. 1938-1947 Revisione di alcune specie de G. actinomyces. Mycopathologia, 1 : 68-76; $2: 84-106 ; 4: 64-84$.

3. BALdACCI, E. 1939-1944 Contributo alla sistematica degli Attinomiceti. Atti Ist. Botan. Pavia, 11(4) : 191-231; 19: 99-129; $\$(5)$ : 139-193.

4. Baron, A. L. 1950 Handbook of Antibiotics. Reinhold Publishing Corp., New York.

5. Beijerinck, M. W. 1913 Ueber Schröter und Cohn's Lakmusmicrococcus. Folia Microbiol., 2: 185-200.

6. Benedict, R. G. 1953 Antibiotics produced by actinomycetes. Botan. Rev., 19: $229-320$.

7. Benedict, R. G., And Lindenfelsen, L. A. 1951 Production of soluble pigments by certain strains of Streptomyces griseus. Antibiotics \& Chemotherapy, 1: $512-517$.

8. Berestneff, N. 1897, 1898 Die Aktinomykose und ihre Erreger. Diss. Moskau; Z. Hyg., 29: 94-116.

9. Bergey's Manual of Determinative Bacteriology, 6th Ed. 1948 The Williams \& Wilkins Co., Baltimore.

10. Brian, P. W. 1951 Antibiotics produced by fungi. Botan. Rev., 17: 357-430.

11. BURKHOLDER, P. R. 1946 Studies on the antibiotic activity of actinomycetes. J. Bacteriol., 52: 503-504.

12. Carvajal, F. 1946 Biologic strains of Streptomyces griseus. Mycologia, 38: 596-607.

13. Сон, , F. 1875 Untersuchungen über Bakterien. II. Beitr. Biol. Pflanz., 1(3): 141-204.

14. DuchE, J. 1934 Les Actinomyces du Groupe Albus. Paul Lechevalier \& Fils, Paris. Encyclopedie Mycologique. VI.

15. Dulaney, D. L. 1948 Observations on Streptomyces griseus. Nitrogen sources for growth and streptomycin production. J. Bacteriol., 56: 305-313.

16. Ehrlich, J., Gottlieb, D., Burkholder, P. R., Anderson, L. E., and Pridham, T. G. 1948 Streptomyces venezuelae, n. sp., the source of chloromycetin. J. Bacteriol., 56: $467-477$.

17. ERIKSON. D. 1935 The pathogenic aerobic organisms of the actinomyces group. Med. Research Council Spec. Rept. Ser. No. 203, London.

18. Erickson, D. 1940 Pathogenic anaerobic organisms of the actinomyces group. Med. Res. Council Spec. Rept. Ser. No. 240, London.

19. Erickson, D. 1941 Studies on some lake-mud strains of Micromonospora. J. Bacteriol., 41: 277-300.

20. Florey, H. W., Chain, E., Heatley, N. G., Jennings, M. A., Sanders, A. G., Abrahay, E. P., and Florey, M. E. 1949 Antibiotics. Oxford University Press. 
21. Gasperini, G. 1892 Recherche morphologiche e biologiche sul genere Actinomyces-Harz. Ann. Igiene, n.s., 2: 167-229.

22. Gadse, G. F. 1946 Litmocidin, a new antibiotic substance produced by Proactinomyces cyaneus. J. Bacteriol., 51: 649-653.

23. Gilbert, A. 1904 Über Actinomyces thermophilus und andere Actinomyceten. Z. Hyg., 47: 383-406.

24. Gratia, A., and Dath, S. 1923-1926 Del'action bacteriolytiques des streptothrix. Compt. Rend. Soc. Biol., 91: 1442-1443; 92: 461, 1125-1126; 93: 451; 94: 1267.

25. Harz, C. O. 1877-1878 Actinomyces bovis, ein neuer Schimmel in den Geweben des Rindes. Jahr. Münch. Tierarzeneische., p. 125-140.

26. Jensen, H. L. 1930-1932 The genus Micromospora Ørskov, a little known group of soil microorganisms. Proc. Linnean Soc. N. S. Wales, 55: 231-249; 57: 175-180.

27. Karel, L., And Roach, E. S. 1951 A dictionary of antibiotics. Columbia University Press, New York.

28. Kelner, A. 1949 Studies on the genetics of antibiotic formation: The induction of antibiotic-forming mutants in actinomycetes. J. Bacteriol., 57: 73-92.

29. Klieneberger-Nobel, E. 1947 The life cycle of sporing Actinomyces as revealed by a study of their structure and septation. J. Gen. Microbiol., 1: 22-32.

30. Krainsky, A. 1914 Die Aktinomyceten und ihre Bedeutung in der Natur. Centrbl. Bakt. II, 41: 649-688.

31. Krassilnikov, N. A. 1938 Ray fungi and related organisms. Actinomycetales. Izv. Akad. Nauk, USSR, Moskau.

32. Krassilnikov, N. A. 1941 The guide to the ray fungi. Actinomycetales. Izv. Akad. Nauk, USSR, Moskau.

33. Krassilnikov, N. A., and Koreniako, A. I. 1939 The bactericidal substance of the actinomycetes. Microbiology (USSR), 8: 673-685.

34. KRISs, A. 1940 On the lysozyme of actinomycetes. Microbiology (USSR), 9: 32-38.

35. Landerkin, G. B., and Lochhead, A. G. 1948 A comparative study of the activity of fifty antibiotic actinomycetes against a variety of soil bacteria. Can. J. Research, 26: 501-506.

36. Lieske, R. 1921 Morphologie und Biologie der Strahlenpilze. G. Borntraeger, Leipsig.

37. Millard, W., ANd BURR, S. 1926 A study of twenty-four strains of actinomyces and their relation to types of common scab of potato. Ann. Appl. Biol., 1s: $58-64$.

38. Nakhimovskaia, M. 1937 The antagonism between actinomycetes and soil bacteria. Microbiology (USSR), 6: 131-157.

39. Окамі, Y. 1949,1950 Studies on the characters of antibiotic streptomyces. III. Japan. Med. J., 2: 203-206; V. Ibid. s: 205-211.

40. Orskov, J. 1923 Investigations into the morphology of the ray fungi. Levin and Munksgaard, København.

41. ОкI, N., ANd HAta, T. 1952 Studies on the substance produced by actinomyces which inhibits homologous strain. J. Antibiotics (Japan), 5: 16-23.

42. Pridham, T. G., and Gotrlieb, D. 1948 The utilization of carbon compounds by some actinomycetales as an aid for species determination. J. Bacteriol., 56: $107-114$.

43. Rouatt, J., Lechevalier, M. P., and Waksman, S. A. 1951 Distribution of antagonistic properties among actinomycetes isolated from different soils. Antibiotics \& Chemotherapy, 1: 185-192. 
44. Schatz, A., Bugie, E., and Waksman, S. A. 1944 Streptomycin, a substance exhibiting antibiotic activity against gram-positive and gram-negative bacteria. Proc. Soc. Exptl. Biol. Med., 55: 66-69.

45. Schatz, A., and Waksman, S. A. 1945 Strain specificity and production of antibiotic substances. IV. Variations among actinomycetes, with special reference to Actinomyces griseus. Proc. Natl. Acad. Sci. U. S., 31: 129-137.

46. Tsiklinsky, P. 1899 Sur les mucédinées thermophiles. Ann. Inst. Pasteur, 18: 500-505.

47. UESAKA, I. 1950, 1952 Studies on the antibiotic action of nocardia. I. Isolation and classification of antibiotic actinomycetales. J. Antibiotics (Japan), 3: 730$735 ; 5: 75-79$.

48. Waksman, S. A. 1916 Bacteria, actinomyces and fungi in the soil. J. Bacteriol., 1: 101 .

49. Waksman, S. A. 1919 Cultural studies of species of actinomyces. Soil Sci., 8: 71-215.

50. Waksman, S. A. 1947 Microbial antagonism and antibiotic substances, 2nd Ed. The Commonwealth Fund, Waltham, Mass.

51. Waksman, S. A. 1950 The actinomycetes: their nature, occurrence, activities, and importance. (Annales cryptogamici et phytopathologici, Vol. 9). Chronica Botanica Co., Waltham, Mass.

52. Waksman, S. A., And Curtis, R. E. 1916 The actinomyces of the soil. Soil Sci., 1: 99-134.

53. Waksman, S. A., And Henrici, A. T. 1943 The nomenclature and classification of the actinomycetes. J. Bacteriol., 46:337-341.

54. Waksman, S. A., Horning, E. S., Welsch, M., And Woodruff, H. B. 1942 Distribution of antagonistic actinomycetes in nature. Soil Sci., 54: 281-296.

55. Waksman, S. A., Schatz, A., And Reynolds, D. M. 1946 Production of antibiotic substances by actinomycetes. Ann. N. Y. Acad. Sci., 48: 73-85.

56. Waksman, S. A., ANd Woodruff, H. B. 1940 Bacteriostatic and bactericidal substances produced by a soil Actinomyces. J. Bacteriol., 40: 609-614.

57. Waksman, S. A., ANd Woodruff, H. B. 1942 Streptothricin, a new selective bacteriostatic and bactericidal agent, particularly active against gram-negative bacteria. Proc. Soc. Exptl. Biol. Med., 49: 207-210.

58. WeLsCH, M. 1941-1942 Bactericidal substances from sterile culture media and bacterial cultures; with special reference to the bacteriolytic properties of actinomycetes. J. Bacteriol., 42: 801-814; 44: 571-588. 



\section{Index to Species of Actinomycetes}

Actinomyces

bovis, 37,161

israeli, 37, 161

\section{Micromonospora}

bicolor, 36

chalcea, 36, 127

coerulea, 35, 36, 128

elongata, 36

fusca, 36,127

globosa, 35, 36, 128

monospora, 36

parva $, 36,128$

vulgaris, 36

\section{Nocardia}

acidophilus, 214

actinoides, 29

actinomorpha, 34, 135, 153

africana, 34, 156

alba, 34, 153

albicans, 30, 33, 146

alni, 30

anaerobicus, 30

asteroides, 31, 32, 132

blackwellii, 34, 150

bovis, 30

caprae, 32, 136

caviae, 34,152

citrea, 33, 148

coeliaca, 33, 135, 144, 153, 218

corallina, 33, 133, 134, 140, 141, 142, 144

cuniculi, 34, 151

cyanea, 210

elaeagnii, 30

erythropolis, 32,134

farcinica, 32, 132

flava, 33, 147

flavescens, 31, 34, 154, 217

fordii, 35,159

freeri, 31

fructifera, 34,155

gabritschewski, 31 gardneri, 34, 159, 222

gedanensis, 30

gibsonii, 34, 155

globerula, 33, 141

israeli, 30

kuroishi, 35, 160, 215

leishmanii, 32, 135

lignieresi, 30

lutea, 34, 149

maculata, 34,158

madurae, 34, 148

mesenterica, 33, 146

minima, 33, 138, 140

muris, 30

myricae, 30

nigra, 34, 149

oligocarbophilus, 30

opaca, 32, 134, 144

parafinae, $30,33,139$

pelletieri, 34, 157

polychromogenes, $31,32,133,134$

pretoriana, 32, 137

pulmonalis, 33, 138

putoriae, 32

pyogenes, 32

rangoonensis, 34, 151

rhodnii, 34, 158

rubra , 33, 99, 143, 156

rubropertincta, 33, 143

salmonicolor, 33, 142, 144

sendaiensis, 32

somaliensis, 31

transvalensis, 33,145

vacciniae, 33, 137

variabilis, 31

viridis, $32,33,147$

\section{Streptomyces}

abikoensum, 14, 79, 185

achromogenes, 12, 59, 226

acidophilus, 17, 29, 100

albidoflavus, 11, 29, 41, 49, 50

albidus, 15, 29, 84 
Streptomyces-cont.

alboflavus, 16, 25, 41, 50, 95

alboniger, 14, 78, 222

albosporeus, 16, 27, 97, 232

albus, $6,9,21,28,38,39,41,49,58,84$,

$85,185,203,231$

almquisti, 84

annulatus, 9, 29, 40

antibioticus, 12, 20, 21, 25, 65, 71, 185,

$186,188,201$

aurantiacus, $7,11,27,53$

aureofaciens, $10,26,49,200$

aureus, 13, 25, 70, 71, 194

beddardii, 19, 121

bikiniensis, 12, 63, 228

bobiliae, 11, 27, 53

bovis, 6

cacaoi $15,89,197$

californicus, 10, 29, 41, 44

candidus, 8, 16, 55, 94

canescus, 12, 56, 196

carnosus, 17, 105, 111

casei, 17, 28, 103

cellulofiavus, 11, 51, 231

cellulosae, 10, 25, 47

chromogenus, 58

chrysomallus, 14, 82, 187

cinnamonensis, 12,60

cinnamoneus, 15, 85, 201

circulatus, 9, 14, 76

citreus, $14,25,81$

clavifer, 17, 103, 111

coelicolor, 6, 9, 29, 41, 126, 202

collinus, 13,71

coroniformis, 19, 114

craterifer, 17, 105

cretaceus, 8

cyaneus, $6,10,42$

cylindrosporus, $8,13,68$

dentrificans, 26

diastaticus, 11, 56, 59

diastatochromogenes, 12, 63

erythreus, 11, 27, 55, 204

erythrochromogenes, $13,71,226$

exfoliatus, 15, 90, 205

farinosus, 9, 15, 84

fasciculatus, 190

fasciculus, 11, 21, 54, 217

felleus, 12, 58, 221

fimbriatus, 17, 104

fimicarius, 12, 57, 59 flaveolus, 10, 26, 45, 205

flavochromogenes, 12, 26, 63

flavogriseus, 11, 55

flavovirens, 11, 50

flavoviridis, 26

flavus, $6,7,14,21,24,25,77,180,187$

flocculus, 16, 95

floridae, 233

fradiae, 8, 16, 21, 27, 88, 97, 206, 214, 215

fulvissimus, 14, 82

fumosus, 9, 16, 92

galtieri, 19,118

gedanensis, 15,86

gelaticus, 15, 28, 90

glaucus, 6, 15, 91

globisporus, $8,9,29,39$

globosus, $8,13,29,68$

gougeroti, 15, 83

gracilis, 7, 18, 106

graminearis, 25

griseocarneus, 12, 65, 86, 209

griseoflavus, 10, 26, 48, 207

griseolus, 11, 21, 54

griseoluteus, 11, 52, 208

griseus, $3,4,6,15,21,23,24,87,107$, $126,166,198,202,207,211,224,227$, 228

hachijoensis, 232

halstedii, 8, 16, 92, 198

hominis, 7

hortonensis, 19, 120

hygroscopicus, 16, 26, 93

intermedius, 19, 116

ipomoea, 19, 116

juniceus, 233

kimberi, 20, 122

kitasatoensis, 210

krainskii, 26

lacertae, 7

lavendulae, $6,13,20,21,22,72,189$,

$191,195,203,209,221,227,229$

Leben, Stessel and Keitt, 16, 96

lieskei, 11, 50

limosus, 11, 51

lipmanii, 16, 41, 93, 102, 192

listeri, 19, 118

loidensis, 18, 108, 110

longisporoflavus, 16, 26, 94

longispororuber, 17, 99

longisporus, 8, 9, 39 
longissimus, 9, 15, 87

maculatus, 19, 113

madurae, 7

marginatus, 18, 108, 112

melanocyclus, $7,17,100$

microflavus, $15,26,81,85,88$

mirabilis, 12, 64, 212

netropsis, 13, 75, 216

niger, $7,14,78$

nigrificans, 8

nitrosporeus, 55, 217

noursei, 12, 59, 218, 220

novacaesareae, 15,89

odorifer, 14, 79

oidiosporus, $9,17,99$

olivaceus, $15,26,88$

olivochromogenus, 12, 61

omiyaensis, 200

panjae, 20, 124

parvus, 10, 46, 186

phaeochromogenus, 13, 69, 73, 212

phaeofaciens, 219

phenotolerans, 7

pluricolor, 6, 10, 42

poolensis, 19, 107, 117

praecox, 18, 107

praefecundus, 18,108

purpeochromogenus, 13, 69

purpurescens, 16,98

putrificans, 14, 28, 81

resistomycificus, 12, 62, 223

reticuli, 9, 13, 74, 209, 225

reticulus-ruber, 227

rimosus, 10, 26, 47, 219, 225

rochei, 9, 40, 96

roseochromogenus, 12, 60, 61, 225

roseodiastaticus, 27

roseoflavus, 14, 80, 205, 213

roseus, $27,28,230$

ruber, $7,14,21,26,27,77,99,156$

rubescens, 17, 101, 185

rubrireticuli, 14, 76 rutgersensis, 16, 91

salmonicolor, $8,18,113$

sampsonii, 19, 111, 115

scabies, 8, 18, 29, 107, 111

setonii, 7, 18, 26, 107

somaliensis, 20, 123

spiralis, 19, 111, 114

spumalis, 8

sterilis, 20, 125

sterilis albus, 20

sterilis flavus, 20

sterilis ruber, 20

sterilis viridis, 20

sulfureus, 25

tanashiensis, 13, 25, 70, 210

tenuis, 18, 108, 111

thermodiastaticus, 17, 28, 102

thermofuscus, 17, 26, 103

thermophilus, 17, 25, 102, 103, 230

totschidlowskii, 6

tumuli, 19, 107, 117

upcottii, 19, 119

venezuelae, $13,22,72,200$

verne, $10,43,102$

verticillatus, 9, 14, 75

vinaceus, 10, 42, 233

violaceoniger, $7,15,86$

violaceus, $6,10,43,213$

virgatus, $8,10,45$

virginiae, 13, 73, 189

viridans, $8,10,44$

viridis, 8, 17, 29, 101, 102, 109

viridochromogenes, $7,13,68$

viridoflavus, 12, 66

viridogenes, 18, 102, 109

wedmorensis, 18,110

willmorei, 20, 124

xanthophaeus, 10, 46

Thermoactinomyces

monospora, 37,130

thalpophilus, 37, 131

vulgaris, 37, 130 



\section{Index to Antibiotics of Actinomycetes}

Abikoviromycin, 79, 101, 177, 184, 185

Achromoviromycin, 59

Achromycin, see Puromycin

Actidione, see Cycloheximide

Actinochrysin, see Actinomycin C

Actinoflavin, 171, 187

Actinomycelin, 172, 179, 185

Actinomycetin, 28, 39, 166, 173, 184, 185

Actinomycin, 46, 65, 77, 166, 168, 171, 179, 183,186

Actinomycin A, 171, 186

Actinomycin B, 171, 186, 188

Actinomycin $\mathrm{C}\left(\mathrm{C}_{1}, \mathrm{C}_{2}, \mathrm{C}_{3}\right), 83,171,179$, 187

Actinomycin $\mathrm{J}_{1}, 171,179,187,188$

Actinomycin X, 171, 186

Actinone, 176, 184, 188

Actinorhodin, 171, 179, 188

Actinorubin, 174, 182, 189

Actinozyme, 173, 184

Actithiazic acid, 61, 74, 172, 178, 189

Albomycin, 236

Amicetin, 172, 178, 190

Antibiotic 136, 174, 182, 191

Antibiotic 185, see Framycetin

Antibiotic 3510,175

Antibiotic $\mathrm{EI}_{6}, 182,191$

Antibiotic HA-9, 181, 192

Antibiotic SKCC 1377, 171, 179, 191

Antibiotic X-206, 172, 180, 192

Antibiotic X-464, 172, 180, 193

Antibiotic X-537A, 172, 180, 193

Antimycin A, 176, 183, 194

Antimycoin, 70, 176, 183, 194, 218

Antiphlei factor, 171, 178, 194

Antismegmatis factor, $173,178,195$

Antitumor factors, 178, 195

Antitumor substance 289, 178, 195

Antiyeast factors, 177

Ascosin, 57, 176, 183, 196

Aureomycin, see Chlortetracycline

Aureothricin, see Thiolutin

Borrelidin, 41, 177, 178, 196
Cacaomycetin, 89, 177, 184, 197

Camphomycin, 172, 178, 183, 197

Candicidin, 23, 87, 166, 176, 183, 198

Candicidin A, 176

Candicidin B, 176

Carbomycin, $92,165,169,175,179,180$, 198

Cardicin, 172, 180, 199

Catenulin, 174, 182, 199

Chloramphenicol, $73,165,169,176,181$, 200,204

Chloromycetin, see Chloramphenicol

Chlortetracycline, 49, 165, 175, 181, 184, 200

Chromin, 176, 183, 201

Cinnamycin, 85, 173, 180, 201

Coelicolorin, 41, 171, 180, 202

Cremomycin, 236

Cycloheximide, 24, 165, 166, 176, 184, 202

Dextromycin, 174, 182, 202

Dihydrostreptomycin, 165, 173, 203, 209

Duomycin, see Chlortetracycline

Ehrlichin, 72, 177, 184, 203

Endomycin, 39, 176, 183, 203

Erythrocin, see Erythromycin

Erythromycin, 55, 165, 169, 175, 179, 204

Exfoliatin, 172, 178, 205

Farcinicin, see Thiolutin

Flavacid, 236

Flaveolin, 174, 181, 205

Flavomycin, 81, 174, 182, 205, 215

Fradicin, 97, 176, 183, 206

Framycetin, 174, 182, 206

Fungicidin, see Nystatin

Fungicidin RAW, see Antimycoin

Fuscamycin, 236

Grisein, 23, 87, 166, 175, 180, 207

Griseoflavin, 173, 180, 207

Griseolutein, 53, 175, 181, 208 
Helixins, 97, 176, 183, 208

Hydroxystreptomycin, 66, 173, 182, 209

Ilotycin, see Erythromycin

Lavendulin, 174, 182, 209

Leucomycin, 175, 179, 209

Litmocidin, 171, 180, 210

Luteomycin, 70, 71, 172, 178, 181, 195, 210

Magnamycin, see Carbomycin

Mannosidostreptomycin, 166, 173, 182, 211

Microcin A, B, 176, 182, 183, 211

Micromonosporin, 128, 173, 180, 212

Miramycin, 64, 212

Moldin, 177, 184, 212

Musarin, 176, 183, 213

Mycelin, 177, 183, 213

Mycetin, 41, 173, 179, 213

Mycobacidin, see Actithiazic acid

Mycomycin, 172, 180, 183, 214

Neamine, 174, 214

Neomycin, 75, 97, 165, 168, 174, 182, 205, 215

Neomycin A, see Neamine

Neomycin B, 174, 215

Neomycin C, 174, 214

Neonocardin, 175, 180, 215

Netropsin, 75, 172, 181, 216

Nigericin, 171, 183, 216

Nitrosporin, 172, 180, 217

Nocardamin, 171, 178, 217

Nocardianin, 172, 179, 218

Nocardin, 173, 178, 218

Nystatin, 60, 176, 183, 218

Orientomycin, 236

Oxytetracycline, 48, 165, 175, 181, 184, 219

Phaeofacin, 177, 184, 219

Phagolessin, 177, 184, 220

Phalamycin, 60, 171, 179, 220

Picromycin, 58, 172, 180, 221

Pleocidin, 174, 182, 221, 229

Pleocidin A, 174, 221
Podomycin, 236

Proactinomycins A, B, C, 159, 172, 180, 222

Puromycin, 79, 177, 178, 222

Resistomycin, 62, 171, 179, 223

Reticulin, 182, 209

Rhodocidin, 171, 179, 181, 223

Rhodomycetin, 23, 171, 179, 224

Rhodomycin, 98, 171, 179, 224

Rimocidin, 48, 177, 183, 225

Roseomycin, 60, 174, 182, 225

Rotaventin, 177, 184, 225

Sarcidin, 172, 179, 226

Sarkomycin, 178, 226

Soframycin, see Framycetin

Streptin, 77, 174, 182, 227

Streptocin, 24, 166, 177, 184, 227

Streptohydrazid, see Streptomycin-Isoniazid complex

Streptolin A, B, 174, 227

Streptomycin, 23, 24, 64, 87, 165, 166, 168, $173,182,203,228$

Streptomycin B, see Mannosidostreptomycin

Streptomycin II, see Streptomycin

Streptomycin-Isoniazid complex, 173, 228

Streptothricin, 72, 166, 168, 174, 182, 183, 229

Streptothricin BI, see Neomycin C

Streptothricin BII, see Neomycin B

Streptothricin VI, 174, 229

Sulfactin, 173, 180, 230

Terramycin, see Oxytetracycline

Thermomycin, 173, 179, 230

Thioaurin, 175, 181, 230

Thiolutin, 39, 51, 175, 182, 184, 231

Thiozolidone, 61

Trichomycin, 177, 184, 232

Vinacetin, 173, 178, 232

Vinactin A, B, C, 43, 175, 178, 233

Viocin, see Viomycin

Viomycin, 43, 45, 165, 169, 175, 178, 233

Xanthomycins, 171, 180, 233 



